\title{
Development of Graphene and Graphene-Nanoparticle Composites for Sensor Applications
}

Saurabh Chaudhari

Follow this and additional works at: https://researchrepository.wvu.edu/etd

\section{Recommended Citation}

Chaudhari, Saurabh, "Development of Graphene and Graphene-Nanoparticle Composites for Sensor Applications" (2015). Graduate Theses, Dissertations, and Problem Reports. 5338.

https://researchrepository.wvu.edu/etd/5338

This Dissertation is protected by copyright and/or related rights. It has been brought to you by the The Research Repository @ WVU with permission from the rights-holder(s). You are free to use this Dissertation in any way that is permitted by the copyright and related rights legislation that applies to your use. For other uses you must obtain permission from the rights-holder(s) directly, unless additional rights are indicated by a Creative Commons license in the record and/ or on the work itself. This Dissertation has been accepted for inclusion in WVU Graduate Theses, Dissertations, and Problem Reports collection by an authorized administrator of The Research Repository @ WVU.

For more information, please contact researchrepository@mail.wvu.edu. 


\title{
Development of Graphene and Graphene-
}

\section{Nanoparticle Composites for Sensor Applications}

\author{
Saurabh Chaudhari
}

\author{
Dissertation submitted to the \\ Benjamin M. Statler College of Engineering and Mineral Resources \\ at West Virginia University \\ in partial fulfillment of the requirements for the degree of
}

Doctor of Philosophy

in

Chemical Engineering

Dr. Charter D. Stinespring, Ph.D., Chair

Dr. Robin S. Hissam, Ph.D.,

Dr. John W. Zondlo, Ph.D.,

Dr.Mark A. Jerabek, Ph.D.,

Dr.Edward M. Sabolsky, Ph.D.

Department of Chemical Engineering

Morgantown, West Virginia

2015

Keywords: Graphene, metal nanoparticles, chemical modification, electrical properties

Copyright 2015 Saurabh Chaudhari 


\section{ABSTRACT \\ Development of Graphene and Graphene-Nanoparticle Composites for Sensor Applications \\ Saurabh Chaudhari}

The goal of this research was the synthesis of graphene and graphene nanocomposite for use as sensor materials. This dissertation describes the optimization of a novel approach to the synthesis of few layer graphene films on $\mathrm{SiC}$, the modification of the graphene surface by wet chemical methods, the nucleation of nanoparticles to form graphene-nanoparticle composites, the fabrication of chemoresistive sensor structures from these materials, and the characterization of these surfaces and films.

In this work, the basic graphene synthesis method which uses halogen based plasma etching and ultra-high vacuum annealing (UHVA), has been optimized to reliably produce one, two, and three layer graphene on SiC films. The process has also been extended by replacing the UHVA step with rapid thermal annealing (RTA) in atmospheric pressure argon. Graphene films produced by both methods have been characterized using x-ray photoelectron spectroscopy (XPS), Raman microscopy, and atomic force microscopy (AFM). The UHVA process produces films with halogen-based and possibly some oxygenbased defects, whereas the RTA processes produces exclusively oxygen-based defects which include epoxide, hydroxyl, and carbonyl groups similar to, but at much lower levels, than that observed for graphene oxide (GO). As in the case for $\mathrm{GO}$, the defect density was further reduced by wet chemical surface modification.

Nanoparticles (Ag, Au, Pt, Ir) were attached to these surfaces using solution based methods. The particle diameter and height distributions along with surface coverage were characterized using AFM methods. Key parameters in these studies included solution composition and incubation time. For electrical characterization and sensor testing, two structures were then fabricated using lithography free methods and electron beam evaporation. The first of these structures, referred to as the transmission line method (TLM) structure, was used in the present work for electrical characterization. Using the TLM structure, the electrical properties were characterized using two and four point probe methods. The films exhibited semiconducting behavior which is believed to be due to the opening of a band gap by the halogen- and oxygen-based defects. Using the two and four pint methods, the Schottky barrier height, the carrier density, electrical resistivity, and the carrier mobility were determined. The electrical resistivity was found to have an inverse relationship with number of graphene layers for one, two, and three layer films. The second device structure was a simple interdigitated sensor structure which was passed on to 
other researchers for sensor studies. Overall, reliable and reproducible synthesis and fabrication methods for graphene and graphene-nanoparticle composites have been developed for the next stage of testing and sensor development. 


\section{Acknowledgements}

Last 4 years of my graduation life in West Virginia University has been an educating and amazing journey. I loved every moment of my grad life, which included challenging coursework, research, attending conferences, useful discussion with colleagues and joyful instances with friends. It is now time to acknowledge all of the individuals and sources that have been a crucial influence throughout my graduate career.

First of all, I owe my deepest gratitude to my advisor Dr. Charter D. Stinespring, for his continuous encouragement and guidance throughout my PhD. This dissertation would not have been possible without his intellectual support and the physical resources he made available to me, that made my PhD studies an enriching experience. All the valuable discussions and the training he provided will benefit me throughout my career. I strongly believe that my potential as a professional engineer has increased multifold during my association with Dr. Stinespring. I would also like to thank ORISE, The National Energy Technology Laboratory and the University Coal Research programs for financial support.

I am very much grateful to my committee members Dr. Robin Hissam, Dr. John Zondlo, Dr. Mark Jerabek and Dr. Edward Sabolsky for their encouragement, technical discussions and feedback. Immense thanks to my lab mates Andrew Graves, Jason Miles, Megan Cain and McKenzie Mills for their help and knowledge exchange in this project.

I owe my sincere thanks and dedicate this dissertation to my parents Shobha Chaudhari and Suresh Chaudhari, for all their support and encouragement through my childhood. I would also like to thank my sister Sayali Chaurasiya, brother in law Kunal Chaurasiya and my cute niece Aarohi for their guidance and moral support.

Special thanks to my friends Priyanka Reddy, Lekha Kuchipudi, Siddhita Aparaj, Meghana Ramakumar, Sravanthi Nalamalapu, Arkamitra Kar, Akshitha Gadde, Akshaya Rane, Rutika Bhapkar, Pratik Pednekar, Sanket Joshi, Pallavi Anipindi, Srinath Velaga, Sonali Moon, Manohar Gaddipati, Nagasree Garapati, Ramalaxmi Krishnaswamy, Suvinneth Ramayanam, Bhargavi Thotakura, Sneha Nagabhayru, Aarthi Reddy, and Kiran Chaudhari for all their support and encouragement during my stay away from home.

I would also like to thank, WVU shared research facility faculty members Dr. Kolin S. Brown, Dr. Weiqiang Ding, Harley Hart, Dr. Marcela Redigolo, and WVU faculty and staff for their essential inputs.

Last but not the least I thank God and Amma Bhagvan for blessing me with such wonderful people in my life. 


\section{Table of contents}

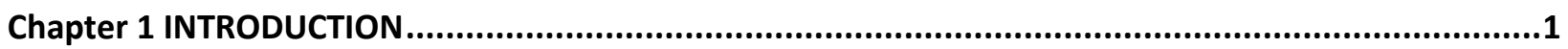

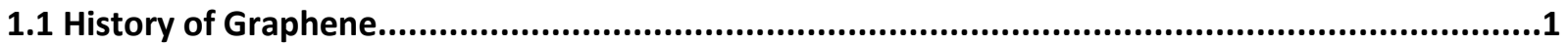

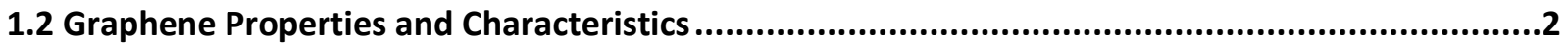

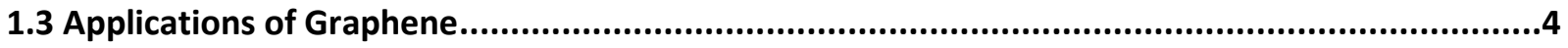

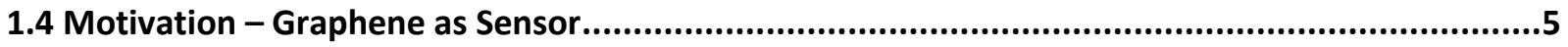

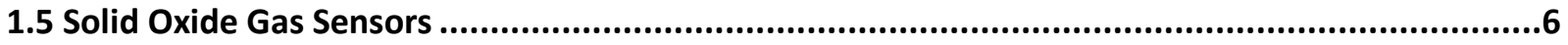

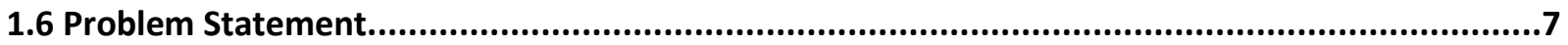

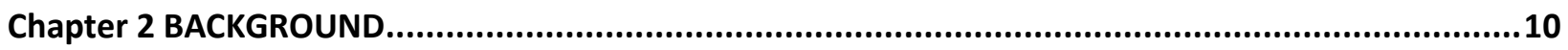

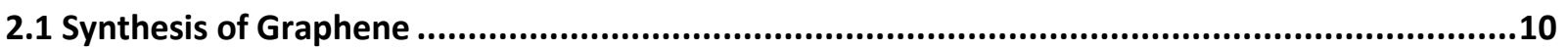

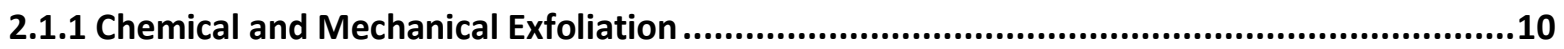

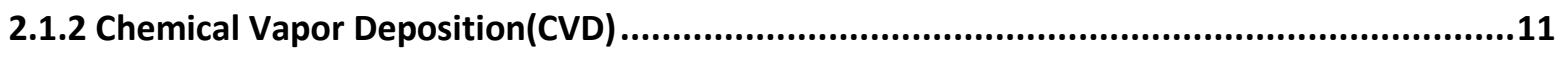

2.1.3 Sublimation of Si from Silicon Carbide ...............................................................12

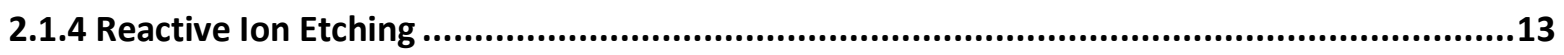

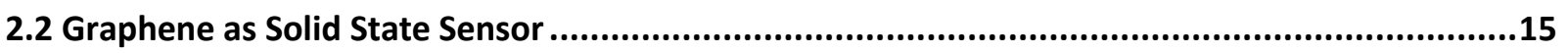

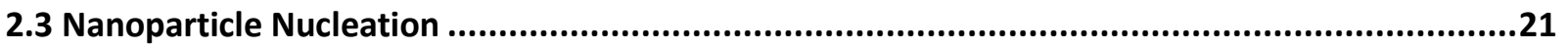

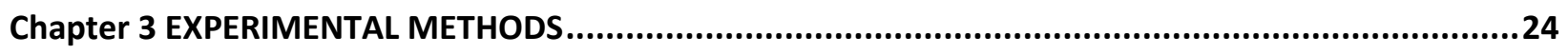

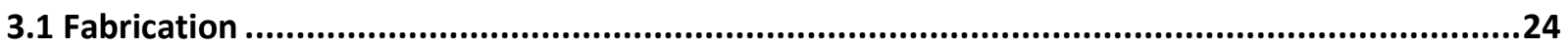

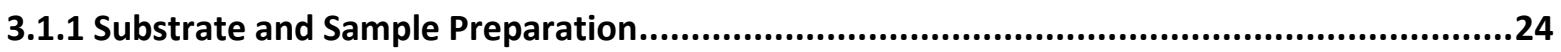

3.1.2 Inductively Coupled Plasma-Reactive Ion Etching (ICP-RIE) System ................................26

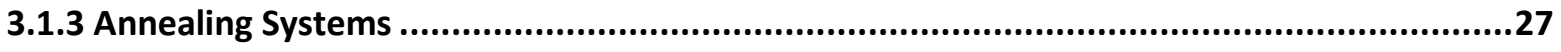

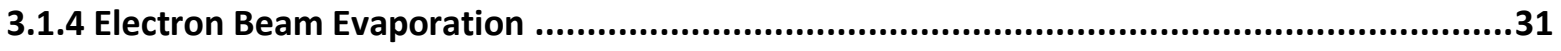

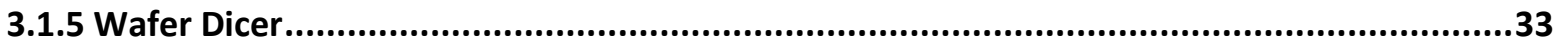

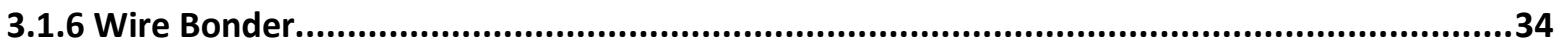

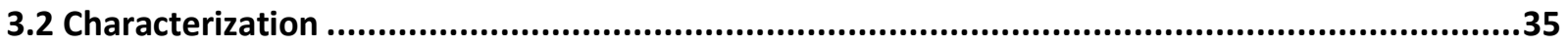


3.2.1 X-ray Photoelectron Spectroscopy (XPS) …...................................................................35

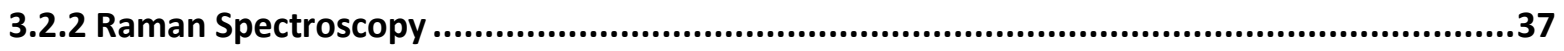

3.2.3 Reflection High Energy Electron Diffraction (RHEED) ..............................................38

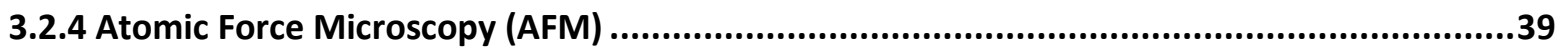

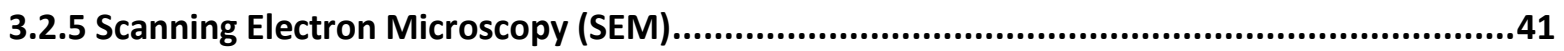

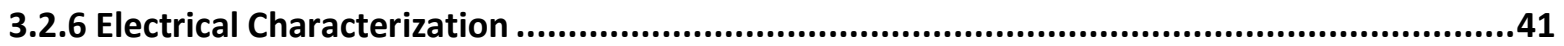

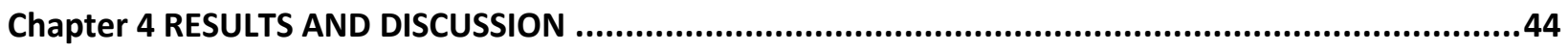

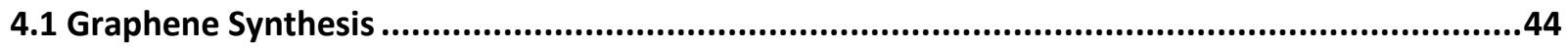

4.1.1 Synthesis Using Ultra High Vacuum Annealing.........................................................44

4.1.2 Synthesis using Rapid Thermal Annealing …...............................................................53

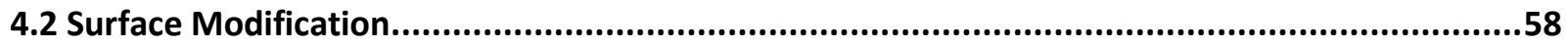

4.3 Nanoparticle Nucleation .................................................................................................61

4.3.1 General Observations of Nanoparticle Nucleation on Graphene ................................61

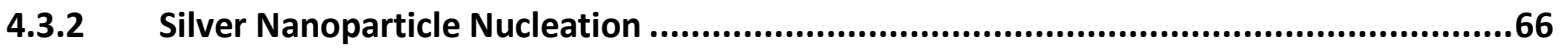

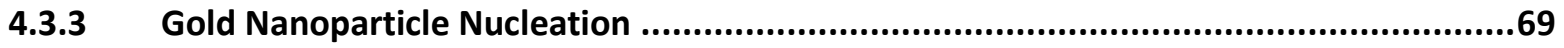

4.3.4 Platinum Nanoparticle Nucleation .........................................................................73

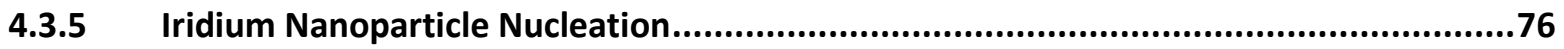

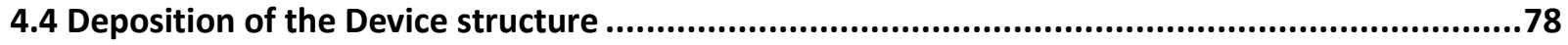

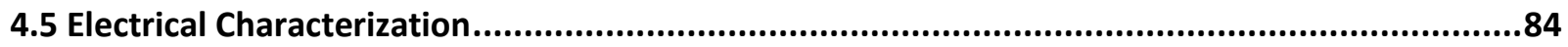

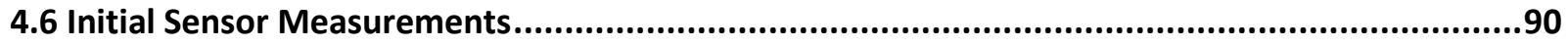

Chapter 5 CONCLUSION AND RECOMMENDATION.....................................................................94

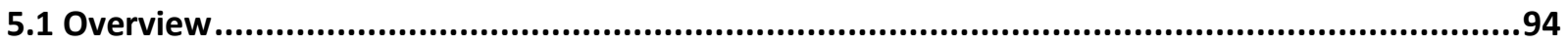

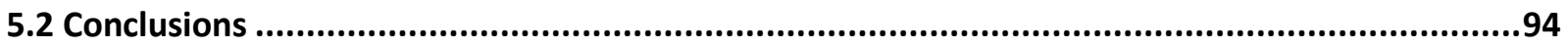

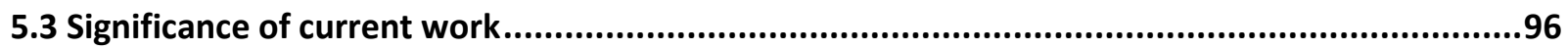

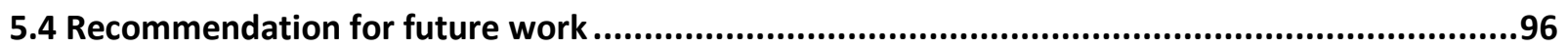

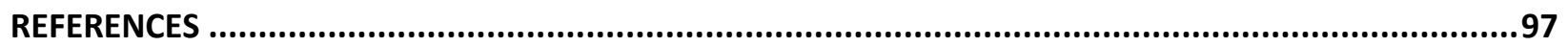




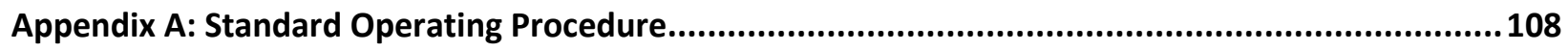

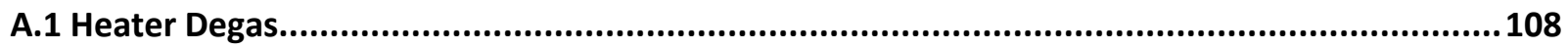

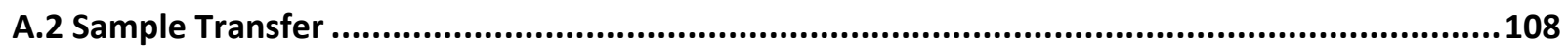

A.3 Load Lock Procedure ....................................................................................................108

A.4 Reflection High Energy Electron Diffraction (RHEED) ................................................109

Appendix B: Recipes for Nanoparticle Nucleation .................................................................111

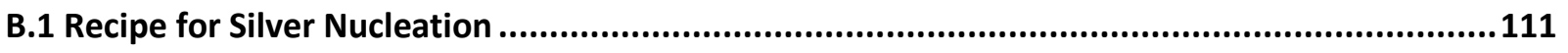

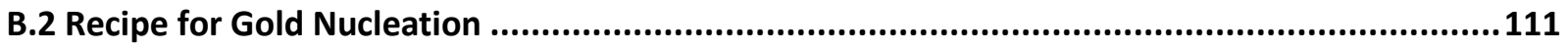

B.3 Recipe for Platinum Nucleation.....................................................................................111

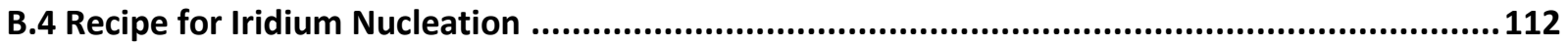

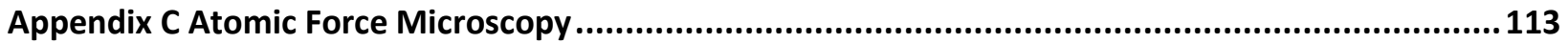

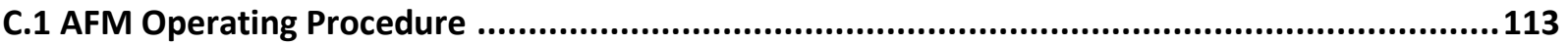

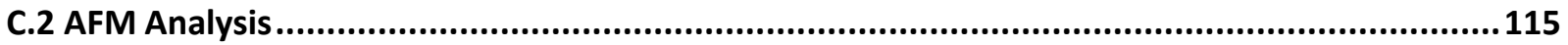

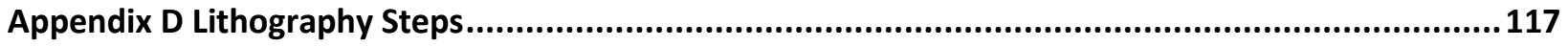

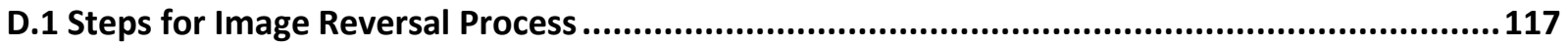

D.2 Steps for Photoresist Strip using Photolithography ............................................................117 


\section{List of figures}

Figure 1.1: Three fold symmetry of graphene [7].

Figure 1.2: Sketch of the electronic structure of graphene, cone-shape linear electronic dispersion and density of states [8]. 3

Figure 1.3: Schematic of graphene-based composite chemo resistive sensor illustrating a dispersed rather continuous layer of nanoparticles.

Figure 2.1: The C1s XPS spectra of: (a) GO, (b) reduced GO [35].

Figure 2.2: (a) The $\mathrm{D}$ and $\mathrm{G}$ spectral region for (i) the $6 \mathrm{H}-\mathrm{SiC}$ substrate ii) a two layer graphene film iii) a pit like defect in two layer film with the optical micrograph inset. (b) The corresponding $2 \mathrm{D}$ spectral region(c) RHEED pattern [46]. 15

Figure 2.3: (a) AFM images of CVD-graphene used for sensors. (b) Response of the defective CVDgraphene, CVD-graphene micro ribbon and $5 \mathrm{~mm}$ wide exfoliated graphene-based sensors to $10^{15}$ molecules of 1, 2-dichlorobenzene [56].

Figure 2.4: The percentile resistance changes of the Ozone treated graphene (OTG) (red) and pristine graphene (black) sensors. The $\mathrm{NO}_{2}$ concentration was modulated from $200 \mathrm{ppm}$ to $200 \mathrm{ppb}$. The inset shows the correlation between percentage response and concentrations, which is in agreement with the langmuir adsorption model [57]. .18

Figure 2.5: (a) SEM image of RGO membrane deposited between electrode arrays. (b) The comparison of the resistance changes between chemically reduced graphene (CRG) (reduced from p-phenylenediamine) sensor and CRG-2 (reduced from hydrazine) sensor at different co concentrations of dimethyl methyl phosphonate [60].

Figure 2.6: (a) Comparison of the responses of RGO-, Sulfonated GO(S-G), and ethylenediamine-modifed RGO(EDA-G)-based sensors toward 50 ppm NO $\mathrm{N}_{2}$ at $1 \mathrm{~V}$ for $10 \mathrm{~min}$. (b) Response of a S-G-based sensor to $50 \mathrm{ppm} \mathrm{NO}, \mathrm{NH}_{3}, \mathrm{H}_{2} \mathrm{O}$ or toluene. (c) Conductance changes of an $\mathrm{S}-\mathrm{-G}$ based sensor during 3 success of exposing to $20 \mathrm{ppm} \mathrm{NO}$ for $10 \mathrm{~min}$ and $\mathrm{N}_{2}$ flow for $30 \mathrm{~min}$. (d) Responses of the S-G-based sensors with different thicknesses of sensing layers after exposure to $50 \mathrm{ppm} \mathrm{NO}$ : $50 \mathrm{~nm}(1), 10 \mathrm{~nm}$ (2), and a few

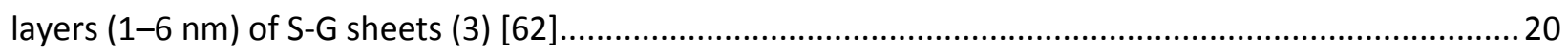

Figure 3.1: Structure of different SiC polytypes (a) 4H-SiC (b) 6H-SiC [82] ............................................2

Figure 3.2: C1s XPS spectra for 6H-SiC (0001) surface after degreasing. .............................................2 26

Figure 3.3: (a) Minilock-Phantom III ICP-RIE system and (b) Schematic of ICP-RIE [86] ..........................27

Figure 3.4: Schematic diagram of the UHV system, built in surface and material studies lab. 28 
Figure 3.5: UHV annealing profile with respect to time.

Figure 3.6: Schematic diagram of a RTA system showing the quartz tube with gas inlet, outlet and thermocouple shield.

Figure 3.7: RTA profile with respect to time. The annealing time was measured from the time the temperature reached at $940^{\circ} \mathrm{C}$ and an average temperature of $953^{\circ} \mathrm{C}$ was obtained for 2 minute anneal.

Figure 3.8: Kurt Lesker E-beam evaporator used to deposit electrical contact in the Shared Research Facilities cleanroom.

Figure 3.9: Temescal BJD200 E-beam evaporator used to deposit electrical contact in the shared Research Facilities cleanroom.

Figure 3.10: DISCO wafer dicer, capable of dicing substrates with great accuracy and minimum contamination. .33

Figure 3.11: Wire bonder used for gold wire bonding between the sensor platform and the packaging. 34 Figure 3.12: The XPS process showing ejection of photoelectron due to incident X-ray, resulting in emission of electron with characteristic kinetic energy [88] ............................................................................. 35

Figure 3.13: Escape depth (mean free path) of electron versus electron kinetic energy [89].................. 36

Figure 3.14: Renishaw micro-Raman spectrometer used, with the $532 \mathrm{~nm}$ green laser and $1 \mu \mathrm{m}$ spot size.

Figure 3.15: Raman spectra comparing of the bulk graphite and monolayer graphene........................... 38

Figure 3.16: RHEED image of as received 6H-SiC (0001) surface after clean taken along 110 ................. 38

Figure 3.17: Schematic diagram of the AFM, showing assembly of laser beam, cantilever, photodetector and substrate surface.

Figure 3.18: Agilent AFM used for understanding the morphology of the surface by imaging at ${ }^{0} \mathrm{~A}$

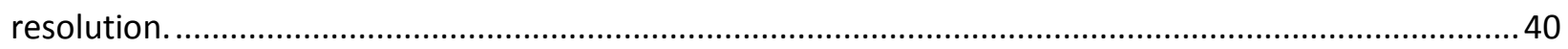

Figure 3.19: Hitachi S4700 Scanning electron microscope …................................................................... 41

Figure 3.20: Two point resistance measurement configuration............................................................ 42

Figure 3.21: Sensor platform for electrical measurement............................................................... 43

Figure 4.1: Profile of the SiC surface showing change in height after one 3 minute $\mathrm{CF}_{4}$ etch cycle. .........45

Figure 4.2: Profile of the SiC surface showing change in height after four 3 minutes of $\mathrm{CF}_{4}$ etch cycles. . 45

Figure 4.3 :Si2s and $\mathrm{C} 1 \mathrm{~s}$ peak for $6 \mathrm{H}-\mathrm{SiC}$, after the $\mathrm{CF}_{4}$ etch and after annealing.................................. 46

Figure 4.4:C1s Spectra of showing the effect of RIE power on number of layers for UHVA graphene samples.

Figure 4.5: Schematic of fluorine- based defects in graphene films. 47 
Figure 4.6: Plot of biased voltage Vs. RIE power and corresponding plot of graphene thickness versus RIE power for $\mathrm{CF}_{4}$ plasma for UHVA samples. 48

Figure 4.7: F 1s XPS spectra for single (a) and three layer (b) graphene films produced by $\mathrm{CF}_{4}$ based ICPRIE and annealing at $970^{\circ} \mathrm{C}$, and (c) a similarly produced five layer graphene after reaction with diazonium. The nominal FWHM of all peaks is $2.0 \mathrm{eV}$. 49

Figure 4.8: RHEED patterns obtained a) before and b) after graphene synthesis. 50

Figure 4.9: Raman spectra for a) the $6 \mathrm{H}-\mathrm{SiC}$ substrate, b) a two layer graphene film, and c) the difference spectrum after scaling and subtracting the $a$ and $b$. .51

Figure 4.10: AFM image for three layer graphene, prepared by UHVA. The roughness of the surface is around $0.352 \mathrm{~nm}$. 53

Figure 4.11: Comparison of the (a) RTA and (b) UHVA survey spectra for three layer graphene film.......53 Figure 4.12:C1s spectra for three layer graphene sample (on left) and corresponding 01s spectra (on right) after 2 minutes of rapid thermal annealing at $950^{\circ} \mathrm{C}$. 54

Figure 4.13: Schematic of oxygen-based defect states, as described for graphene oxide [35]. 55 Figure 4.14: Graph showing number of graphene layers and oxygen concentration determined by XPS with respect to time for RTA samples. .56 Figure 4.15: Plot of biased voltage Vs. RIE power and corresponding plot of graphene thickness versus RIE power for $\mathrm{CF}_{4}$ plasma for RTA samples. .56

Figure 4.16:Raman spectra for a) the $6 \mathrm{H}-\mathrm{SiC}$ substrate, b) a three layer graphene film, and c) the difference spectrum after scaling and subtracting the $a$ and $b$. .57 Figure 4.17: AFM image for three layer graphene, prepared by RTA. The roughness of the surface is around $0.532 \mathrm{~nm}$. .57

Figure 4.18: C1s XPS spectra showings structural characteristics of the graphene film after 12 hour $\mathrm{NaBH}_{4}$ reduction at room temperature. 59 Figure 4.19: C1s XPS spectra showings structural characteristics of the graphene film after $1 \mathrm{hr} \mathrm{HCl}$ reduction at room temperature.

Figure 4.20: C1s XPS spectra showings structural characteristics of the graphene film after $12 \mathrm{hr}$ methanol reduction at room temperature.

Figure 4.21: SEM images of graphene surface after 12 hour incubation period for silver nucleation.......62 Figure 4.22: SEM images of graphene surface after 5 min sonication in acetone for silver nucleation after 12 hour incubation period. 
Figure 4.23: (a) $2 \mu \mathrm{m} \times 2 \mu \mathrm{m}$ AFM image of Au NPs on graphene, b) $500 \mathrm{~nm} \times 500 \mathrm{~nm}$ image of square region in (a) with corresponding lines scan, c) $100 \mathrm{~nm} \times 100 \mathrm{~nm}$ image of square region in (b) and corresponding line scans, and d) schematic of Volmer-Webber growth mechanism .65 Figure 4.24: Change in color from colorless to pale yellow after 12 hours, confirming formation of silver colloid.

Figure 4.25: AFM image of the AgNPs, obtained with $2.71 \mathrm{mM} \mathrm{NaBH}_{4}$ and $10 \mathrm{mM} \mathrm{AgNO}_{3}$, after 3 hours of incubation time, with the histogram showing AgNPs size and height distribution. .67

Figure 4.26: AFM images of the AgNPs, obtained with $2.71 \mathrm{mM} \mathrm{NaBH}_{4}$ and $10 \mathrm{mM} \mathrm{AgNO}_{3}$, after 6 hours of incubation time, with the histogram showing AgNPs size and height distribution.

Figure 4.27: AFM images of the AgNPs, obtained with $2.71 \mathrm{mM} \mathrm{NaBH}_{4}$ and $10 \mathrm{mM} \mathrm{AgNO}_{3}$, after 12 hour of incubation time, with the histogram showing AgNPs size and height distribution. 68 Figure 4.28: SEM images of the AgNPs, obtained with $2.71 \mathrm{mM} \mathrm{NaBH}_{4}$ and $10 \mathrm{mM} \mathrm{AgNO}_{3}$, after 3, 6 and 12 hours of incubation time. .68

Figure 4.29: Change in color from colorless to pale purple, confirming formation of gold colloid............. 70 Figure 4.30: AFM images of the AuNPs, obtained with $2.71 \mathrm{mM} \mathrm{NaBH}_{4}$ and $9.6 \mathrm{mM} \mathrm{HAuCl}_{4}$, after $3 \mathrm{hr}, 6$ $\mathrm{hr}$ and $12 \mathrm{hr}$ of incubation time. 71

Figure 4.31: AFM images of the AuNPs, obtained with $2.71 \mathrm{mM} \mathrm{NaBH}_{4}$ and 12 hour incubation period for 9.6 $\mathrm{mM}, 19.2 \mathrm{mM}$ and $38.4 \mathrm{mM}$ concentrations of $\mathrm{HAuCl}_{4}$ respectively. 72

Figure 4.32: AFM images of the PtNPs, obtained with $2.71 \mathrm{mM} \mathrm{NaBH}_{4}$ and $5 \mathrm{mM} \mathrm{H}_{2} \mathrm{PtCl}_{6}$, after $24 \mathrm{hr}, 36$ $\mathrm{hr}$ and $48 \mathrm{hr}$ of incubation time. 74

Figure 4.33: AFM images of the PtNPs, obtained with $2.71 \mathrm{mM} \mathrm{NaBH}_{4}$ and $24 \mathrm{hr}$ incubation period for 5 $\mathrm{mM}, 10 \mathrm{mM}$ and $20 \mathrm{mM}$ concentration of $\mathrm{H}_{2} \mathrm{PtCl}_{6}$ respectively. .75

Figure 4.34: Change in color from colorless to brown(left) and from drak brown to colorless(right), confirming formation of platinum and Iridium colloid, respectively. 76 Figure 4.35: AFM images of the IrNPs, obtained with $2.71 \mathrm{mM} \mathrm{NaBH}_{4}$ and $5 \mathrm{mM} \mathrm{H}_{2} \mathrm{IrCl}_{6}$, after $12 \mathrm{hr}$ and 24 hr incubation time. 77 Figure 4.36: Photolithography steps for patterning of the graphene films 79 Figure 4.37: Optical micrograph of photoresist with the desired sensor pattern to create contact holes. 80

Figure 4.38: Optical micrographs a) After deposition of Ti/Au contacts, b) After deposition of photoresist to protect the graphene strip during an $\mathrm{O}_{2}$ plasma etch to remove the unwanted graphene, c) after $\mathrm{O}_{2}$ plasma etch and photoresist removal. 
Figure 4.39: Failure of the wire bonds on the graphene surface, resulting in the lift off of gold contacts.

Figure 4.40: Oxygen pattern (left) and device pattern (right) shadow masks used in lithography free fabrication. The dark area represent openings in the sample. 82

Figure 4.41:(a) A series of successful wire bonds made on bonding pads, located on the $\mathrm{SiO}_{\mathrm{x}}$ strip (b) Magnified view of the wire bonds.

Figure 4.42: I-V plot for a) one, b) two, and c) three layer graphene on SiC films and d) the corresponding response for the $\mathrm{SiC}$ substrate.

Figure 4.43: Richardson-Duschman analysis of the two layer I-V data. .86

Figure 4.44: I-V plot for a) one, b) two, and c) three layer graphene on SiC films and d) the corresponding response for the SiC substrate, prepared by RTA. 87

Figure 4.45: I-V plot for a) three layer RTA graphene, b) three layer RTA graphene treated with $1 \mathrm{hr} \mathrm{HCl}$, and c) three layer RTA graphene treated with $12 \mathrm{hr}$ methanol.

Figure 4.46: I-V plot for (a)two layer RTA graphene, (b) silver(c) gold(d) platinum(e) iridum attached two layer RTA graphene films. 89

Figure 4.47: a) Paste-up of the sensor platform and b) The sensor platform in operation. .90

Figure 4.48: a) Sensor platform mounted in the test cell, b) Gas inlet for the test cell, and c) Sensor test unit.

Figure 4.49: Effect of hydrogen composition on I-V characteristics at $100^{\circ} \mathrm{C}$ 91

Figure 4.50: Sensor response to alternate $120 \mathrm{sec}$ pulses of $\mathrm{H}_{2}$ and $\mathrm{Ar}$. 92

Figure 4.51: Chemical response of the graphene sensor to a) $\mathrm{H}_{2}$ and b) $\mathrm{CO}$ as a function of sensor temperature and applied voltage. 


\section{List of Tables}

Table 4.1: AFM Analysis of Silver Nanoparticles for $2.71 \mathrm{mM} \mathrm{NaBH}_{4}$ and $10 \mathrm{mM} \mathrm{AgNO}_{3}$. 69

Table 4.2: XPS analysis for silver nanoparticles for $2.71 \mathrm{mM} \mathrm{NaBH}_{4}$ and $10 \mathrm{mM} \mathrm{AgNO}_{3}$.

Table 4.3: AFM Analysis of Gold Nanoparticles for $2.71 \mathrm{mM} \mathrm{NaBH}_{4}$ and $9.6 \mathrm{mM} \mathrm{HAuCl}_{4}$.

Table 4.4: XPS analysis for silver nanoparticles for $2.71 \mathrm{mM} \mathrm{NaBH}_{4}$ and $9.6 \mathrm{mM} \mathrm{HAuCl}_{4}$. 71

Table 4.5: AFM Analysis for Gold Nanoparticles for $2.71 \mathrm{mM} \mathrm{NaBH}_{4}$ and 12 hour incubation time for 9.6 $\mathrm{mM}, 19.2 \mathrm{mM}$ and $38.4 \mathrm{mM}$ concentrations of $\mathrm{HAuCl}_{4}$.

Table 4.6: XPS Analysis for Gold Nanoparticles for $2.71 \mathrm{mM} \mathrm{NaBH}_{4}$ and 12 hour incubation time for 9.6 $\mathrm{mM}, 19.2 \mathrm{mM}$ and $38.4 \mathrm{mM}$ concentrations of $\mathrm{HAuCl}_{4}$.

Table 4.7: AFM Analysis for Platinum Nanoparticles with $2.71 \mathrm{mM} \mathrm{NaBH}_{4}$ and $5 \mathrm{mM} \mathrm{H}_{2} \mathrm{PtCl}_{6}$, after 24 hour, 36 hour and 48 hour of incubation time.

Table 4.8: XPS analysis with Platinum Nanoparticles $2.71 \mathrm{mM} \mathrm{NaBH}_{4}$ and $5 \mathrm{mM} \mathrm{H}_{2} \mathrm{PtCl}_{6}$, after 24 hour, 36 hour and 48 hour of incubation time. 74

Table 4.9: AFM Analysis for Platinum Nanoparticles with $2.71 \mathrm{mM} \mathrm{NaBH}_{4}$ and 24 hour incubation period for $5 \mathrm{mM}, 10 \mathrm{mM}$ and $20 \mathrm{mM}$ concentration of $\mathrm{H}_{2} \mathrm{PtCl}_{6}$ respectively..... .75

Table 4.10: XPS Analysis for Platinum Nanoparticles with $2.71 \mathrm{mM} \mathrm{NaBH}_{4}$ and 24 hour incubation period for $5 \mathrm{mM}, 10 \mathrm{mM}$ and $20 \mathrm{mM}$ concentration of $\mathrm{H}_{2} \mathrm{PtCl}_{6}$ respectively...... .75 Table 4.11: AFM Analysis for Iridium nanoparticles with $2.71 \mathrm{mM} \mathrm{NaBH}_{4}$ and $5 \mathrm{mM} \mathrm{H}_{2} \mathrm{IrCl}_{6}$, after 12 hour and 24 hour incubation time.

Table 4.12: XPS Analysis for Iridium Nanoparticles with $2.71 \mathrm{mM} \mathrm{NaBH}_{4}$ and $5 \mathrm{mM} \mathrm{H}_{2} \mid \mathrm{rCl}_{6}$, after 12 hour and 24 hour incubation time.

Table 4.13: Electrical properties of the UHV annealed graphene films. .86

Table 4.14: Electrical properties of the RTA graphene films. 87

Table 4.15: Electrical properties of the three layer RTA and chemically modified RTA graphene films. ... 89 Table 4.16: Electrical properties of the three layer RTA and nanoparticle attached RTA graphene films. 90 


\section{Chapter 1 INTRODUCTION}

Graphene is a transparent, two dimensional (2D) form of a monolayer $\mathrm{sp}^{2}$ bonded carbon, arranged in the honeycomb lattice structure. It is a basic building block for graphitic materials like fullerenes (OD), nanotubes (1D) and graphite (3D). Though, it was studied extensively in theory and found to have remarkable electrical, mechanical and chemical properties, instability was an issue, until in 2004, when Geim and Novoselov discovered free-standing graphene [1]. Follow up experiments confirmed the massless Dirac fermions as the charge carriers and opened up a new era of graphene synthesis.

\subsection{History of Graphene}

The path to discovery of graphene traces back to the nineteenth century when English chemist Benjamin recognized the highly layered nature of thermally reduced graphite oxide in 1859 [2]. In 1918, Kohlschutter and Haenni used powder diffraction to study the structure of graphite and graphite oxide paper [3]. P. R. Wallace developed a theory about the electronic properties of graphene in 1947, while investigating properties of graphite [4]. In 1948, Ruess and Vogt used transmission electron microscopy (TEM) to image monolayer and few layer of graphene. Single and multilayer graphene flakes were isolated and studied by Bohem in 1962 using TEM [5].

In 2002, first graphene patent on 'nano-scaled graphene plates' was filed, but it was two years until Andre Geim and Kostya Novoselov at University of Manchester extracted single-atom-thick crystallites from bulk graphite by pulling graphene layers from graphite and transferred them onto thin $\mathrm{SiO}_{2}$ film on a silicon wafer [1]. The process was called micromechanical cleavage or the 'scotch tape method'. The key to their success was high-throughput visual recognition of graphene on a properly chosen substrate, which provides a small but noticeable optical contrast. The duo was awarded with Nobel Prize in 2010 for their discovery.

Following this discovery, the first observation of the anomalous quantum Hall effect in graphene was reported in 2005 by Gusynin [4].This provided direct evidence of graphene's theoretically predicted massless fermions, as reported by Philip Kim and Yuanbo Zhang in 2005 [6]. Since then, the graphene 'gold rush' has begun, in which graphene was studied extensively for the various electronics and optical applications. 


\subsection{Graphene Properties and Characteristics}

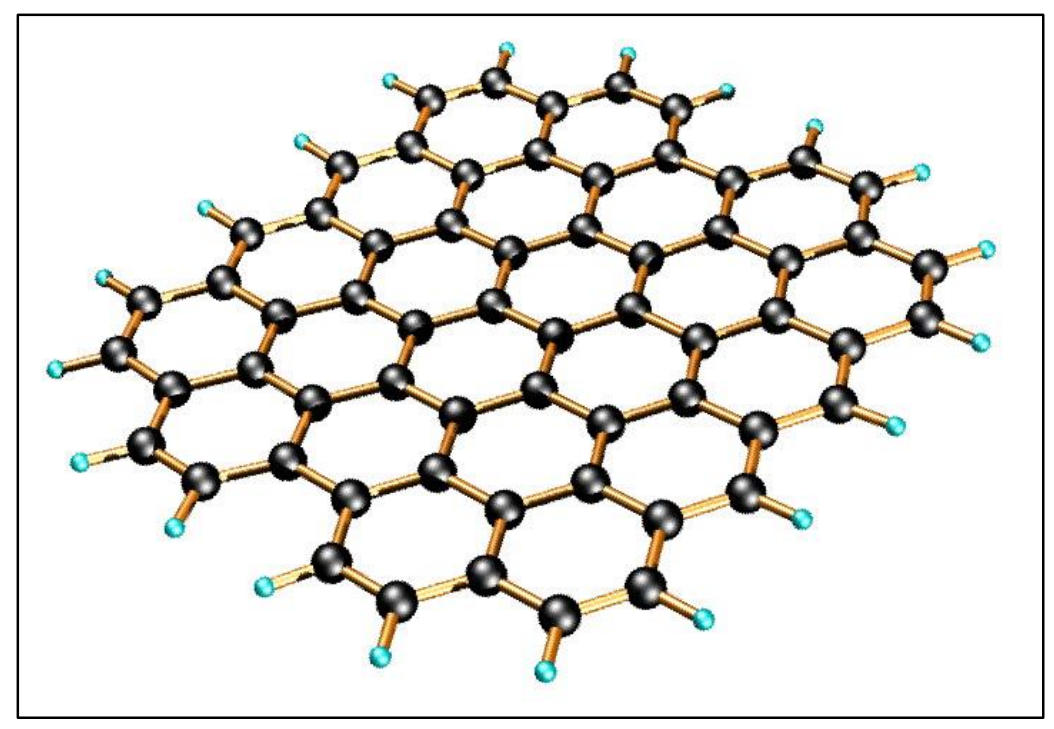

Figure 1.1: Three fold symmetry of graphene [7].

Graphene is a monoatomic two-dimensional structure of carbon atoms. It is an allotrope of carbon and considered as the building block of many carbon allotropes. As seen in the Figure 1.1, the C-atoms in graphene have three fold symmetric covalent bonds [7]. Graphene can be rolled to form a carbon nanotube, a 1D structure of carbon atoms, or it can be wrapped to form fullerene, a OD structure. It can also be stacked to form graphite, a 3D structure of carbon atoms.

In graphene, the atoms are arranged in a hexagonal packed structure due to the $\mathrm{sp}^{2}$ hybridization. This type of lattice is called honeycomb lattice. In $s p^{2}$ hybridization, the $2 s$ state mixes with two of the $2 p$ orbitals to form three different quantum mechanical states or orbitals. Carbon atoms have a total of 6 electrons; 2 in the inner shell and 4 in the outer shell. The 4 outer shell electrons in an individual carbon atom are available for chemical bonding, but in graphene, each atom is connected to 3 other carbon atoms on the two dimensional plane, leaving 1 electron freely available in the third dimension for electronic conduction. These highly-mobile electrons are called pi $(\pi)$ electrons and are located above and below the graphene sheet. These $\pi$ orbitals overlap and help to enhance the carbon to carbon bonds in graphene. Fundamentally, the electronic properties of graphene are dictated by the bonding and anti-bonding (the valance and conduction bands) of these $\pi$ orbitals. In intrinsic (undoped) graphene the Fermi level is situated at the intersection of two cones, as shown in figure 1.2 [8]. Since the density of states of material is zero at that point, the electrical conductivity of intrinsic graphene is quite low. The Fermi level can however be changed by an electric field so that the material becomes either $n$-doped (with electrons) or 
p-doped (with holes) depending on the polarity of the applied field. Graphene can also be doped by adsorbing molecules, for example, water or ammonia on its surface. The electrical conductivity for doped graphene is potentially quite high, at room temperature it may even be higher than that of copper.
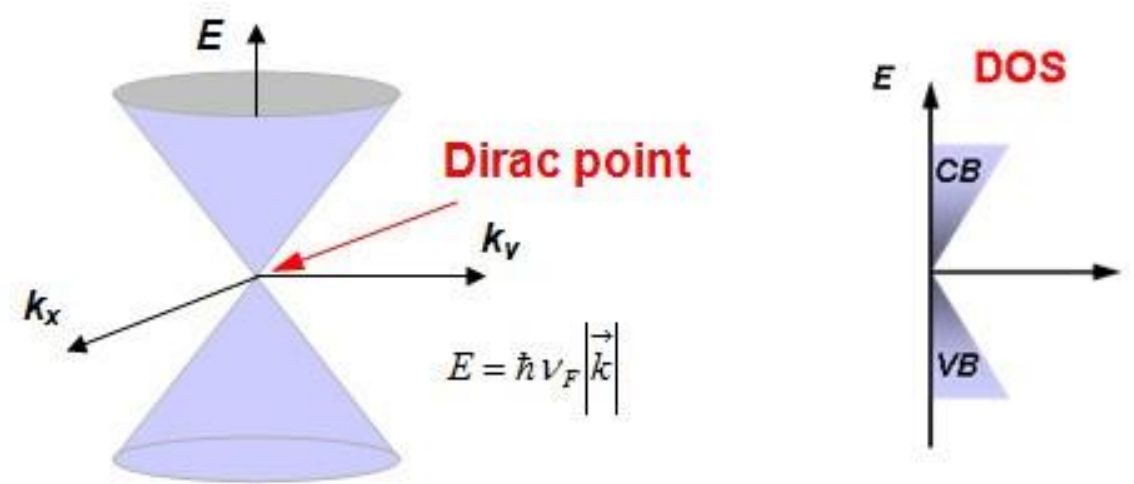

Figure 1.2: Sketch of the electronic structure of graphene, cone-shape linear electronic dispersion and density of states [8].

Graphene has no band gap as the valence band is filled and conduction band is empty in the absence of any external field. But, in the presence of a bipolar electric field, the electrons from the unhybridized $\mathrm{p}$ orbital of graphene rise and fill the conduction band, giving $\mathrm{n}$-type semiconductor behavior. Similar results are obtained when the electric field is applied in the opposite direction, with hole as the charge carriers and p-type semi-conductor behavior. These carriers, being massless and low in number can travel for a long distance without experiencing any collisions and thus have extreme room temperature mobilites of $\sim 200,000 \mathrm{~cm}^{2} /$ V.s and nominal velocity $\sim 1 / 300$ the speed of light. Also, the lack of scattering contributes to graphene's low resistivity [9].

Recent measurements of graphene has shown a Young's modulus of 0.5-1 TPa, a breaking strength of $42 \mathrm{~N} / \mathrm{m}$ and an intrinsic strength of $130 \mathrm{GPa}[10,11]$. This makes graphene one of the strongest materials known. Further, it has been shown that graphene-polymer nanocomposites have a much higher resistance to fracture and crack propagation [12].

For a material to be able to be used in optoelectronic applications, it must be able to transmit more than $90 \%$ of light and also offer resistance less than $1 \times 10^{-6} \Omega-\mathrm{m}$. Graphene is an almost completely transparent material and is able to optically transmit up to $97.7 \%$ of light. It is also highly conductive, which makes it ideal material for optoelectronic applications such as LCD touchscreens for smartphones, tablet and desktop computers and televisions. Indium tin oxide (ITO) is currently used for most of the optical application. However, recent tests have shown that graphene is potentially able to match the properties of ITO, even in current (relatively under-developed) states [13]. Also, it has been reported that 
the optical absorption of graphene can be changed by adjusting the Fermi level. Graphene displays additional properties which can enable very clever technology to be developed in optoelectronics by replacing the ITO with graphene. The fact that high quality graphene has a very high tensile strength, and is flexible, makes it almost inevitable that it will soon become utilized in touchscreen and other applications.

\subsection{Applications of Graphene}

Graphene has good electrical conductivity as well as high surface to mass ratio, which makes it a promising candidate for electrode applications. Due to its high transparency and relatively low sheet resistance, it is also emerging as a potential candidate in photovoltaic applications as a transparent electrode $[14,15,16]$. Su et al. constructed the heterojunction solar cells with Zinc oxide $(\mathrm{ZnO})$ as the buffer layer with conductive graphene oxide (GO) and reduced graphene oxide (RGO) films [17]. It has been also demonstrated that a more conductive chemical vapor deposition (CVD) graphene film with sheet resistance around $250 \Omega /$ sq. and transparency of $72 \%$ shows performance comparable to conventional ITO.

Several studies also demonstrated the use of graphene as electrode for lithium ion batteries. They showed that lithium ions can be intercalate between the graphene layers such that there can be as many as one lithium ion per two carbon atoms. This is significantly higher than conventional graphite. They also store energy in the charged double layer that develops when a voltage is applied between electrodes that are immersed in an electrolyte. Thus graphene can be a superior alternative electrode for use in lithium batteries in applications that require high peak power for short periods of time.

Another application of graphene is in future electronic circuits and devices utilizing high frequency logic circuits and field effect transistors [18]. These devices make use of the zero-band gap feature of graphene and its ambipolar field effect property [1]. Even though zero band gap limits applications, graphene's high mobility compensates for this and draws attention for high frequency devices. Carrier mobilities around $20000 \mathrm{~cm}^{2} /$ Vs have been reported for single, bi and tri-layer graphene films [19, 20]. This unusually high carrier mobility promises fast operating speed for graphene transistors. The saturation velocity $\left(5 \times 10^{7} \mathrm{~cm} / \mathrm{s}\right)$ for graphene is twice as high as that of GaAs and four times that of Si and that makes it valuable material to investigate for electronic systems and devices [21, 22]. 
Graphene suspended on a $\mathrm{Si} / \mathrm{SiO}_{2}$ substrates, actuated by optical or electrical energy, vibrates out of the plane with certain frequency, as shown in studies utilizing graphene as microwave frequency generator [23]. Resonators of this nature can be used to detect minute mass, temperature and many quantum effects due to graphene's high resonant frequencies and lightweight membrane structures.

Advanced composites are increasingly a key component in the design of new windmill blades, aircraft, and other applications requiring ultra-light, high-strength materials. For polymer composites, one of the most important factors is the link between polymers and additives. The wrinkled surface texture of graphene due to the high density of surface defects interlocks extremely well with the surrounding polymer materials. This enhances the interfacial load transfer between graphene and the host materials. The second advantage is surface area. Both the top and bottom surfaces of the graphene sheet can be in close contact with the polymer matrix [12, 24].

In addition to these areas where graphene may find industrial applications, graphene is emerging as a promising candidate in sensing applications, be it in regards to biological or in chemical applications. The single atom thickness and inherently low electrical-noise in graphene could enable ultra-sensitive biological and chemical sensors.

\subsection{Motivation - Graphene as Sensor}

In recent years, chemical and industrial activities have expanded globally. Examples include the petrochemical, transportation and energy sector. These industries have the potential to release toxic substances in the forms of solids, liquids and vapors, which affect the environment and human health. In many cases, the industrial activity required for man's existence may have extremely adverse effects on human and other forms of life. One example of this is acid rain. Acid rain is one of the major problems that affect large parts of the world. Any industrial process that results in the primary emission of sulphur dioxide and nitrogen oxides can be responsible for acid rain. The emissions of these toxic pollutants into the atmosphere represents a severe environmental and health hazard. In order to minimize the adverse impact of industrial activity, effective control strategies and processes must be developed. Sensors are required as an integral part of these strategies and processes for both process control and emission monitoring.

Graphene has the potential to detect a variety of gas molecules [25].The operational principle of graphene devices is based on changes in their electrical resistivity due to gas or liquid phase molecules adsorbed on graphene's surface and acting as donors or acceptors, similar to other solid-state sensors. 
Using graphene as a sensor material has the potential to increase the sensitivity to its ultimate limit and detect individual dopants, because of its characteristics. Graphene is a strictly two-dimensional material and, as such, has its whole volume exposed to surface adsorbents, which maximizes their effect. Graphene exhibits high metallic conductivity and, hence, low Johnson noise even in the limit of no charge carriers, where a few extra electrons can cause notable relative changes in carrier concentration. Graphene has few crystal defects, which ensures a low level of excess $(1 / f)$ noise caused by their thermal switching. All these features form a unique combination that maximizes the signal-to-noise ratio for detecting changes in a local concentration by less than one electron charge at room temperature [25].

The problem with graphene-based sensors is not sensitivity. For example, Schedin has shown that even single molecule adsorption events can be detected under ideal conditions [25]. Rather, the problem

is selectivity, the ability of determining the identity of the adsorbed molecule. One approach to making graphene selective is to modify the surface to make it respond differently to one molecule than another. Given an array of sensors, each modified in different ways, should produce a response signal (fingerprint), representative of a specific target molecule. In the case of biosensors, this is done by attaching a functional group that interacts with only a few target molecules [26]. For the research done in our lab, the approach was to attach nanoparticles to the surface of graphene and thereby modify its adsorption characteristics.

These ideas form the basis of the research performed for this dissertation. Before outlining a detailed problem statement however, it is helpful to review one last topic. Specifically that of metal oxide gas sensors as they relate to the present work.

\subsection{Solid Oxide Gas Sensors}

High temperature, solid oxide gas sensors have been studied extensively in recent years and have a history that extends over five decades [27]. These sensors have various applications such as combustible gas monitoring, oxygen sensing for combustion control, and humidity sensing for living spaces. Extensive studies have established a basis for materials selection for specific gas sensing applications. In addition, numerous studies have established the basic sensing mechanisms and device fabrication techniques. Although, a variety of detection schemes are possible (e.g. capacitance, optical, mass, work function), perhaps the simplest and most relevant here is the chemo-resistor scheme. Here, interaction with the gas changes the resistance or conductivity of the film and provides the basis for detection. The fact that interaction for a specific target molecule is highly dependent on the oxide, provides a basis for sensor selectivity [28, 29]. This behavior was first observed by Brattein et al. and Heiland et al [30, 31]. 
Solid oxide gas sensors, in general, have high sensitivity and selectivity. Without being too simplistic, the problems that arise with these sensors are twofold. First, the conduction mechanisms involve surface, bulk and intergranular conduction. These mechanisms are temperature dependent, so the relative contribution from each changes with operating temperature. This introduces a certain level of complexity, which, in general, can be dealt with and can be used in some situations to advantage. The second difficulty relates to the fact that particle grain size or structure also depend on temperature, and generally, the particle size coarsens with temperature and time. As a result, the conduction mechanism changes (relative distribution between surface, bulk and grain boundary) and the surface area and sensitivity change. The research described here takes advantage of the selectivity of particle-gas interactions, while avoiding the problem of grain coarsening [28, 29, 30, 32, 33].

\subsection{Problem Statement}

As outlined above, graphene sensors have high sensitivity and rapid response times, but they provide no basis for selectivity. Solid oxide sensors provide desired selectivity but have limitations associated with thermally induced particle coarsening and change in conduction mechanism. The overall goal of the research is to investigate the use of graphene and graphene-nanoparticle composite films to produce sensors with the desired sensitivity, response time and selectivity.

The initially proposed structure of these sensors is shown in Figure 1.3. This sensor consists of a few (one, two or three) layer graphene film grown on SiC substrate. When a voltage is applied to the metallic source and drain contact, a current is established, which reflects the resistivity of the graphene film. Changes in resistivity due to gas adsorption can thereby be detected. Nanoparticles attached to the surface modify the adsorption process and, therefore, the change in resistance. Because the nanoparticles are isolated, coarsening cannot occur. Moreover, since the conduction path remains through the graphene and not the particles, temperature dependent changes in the oxide conduction mechanism are not an issue. 


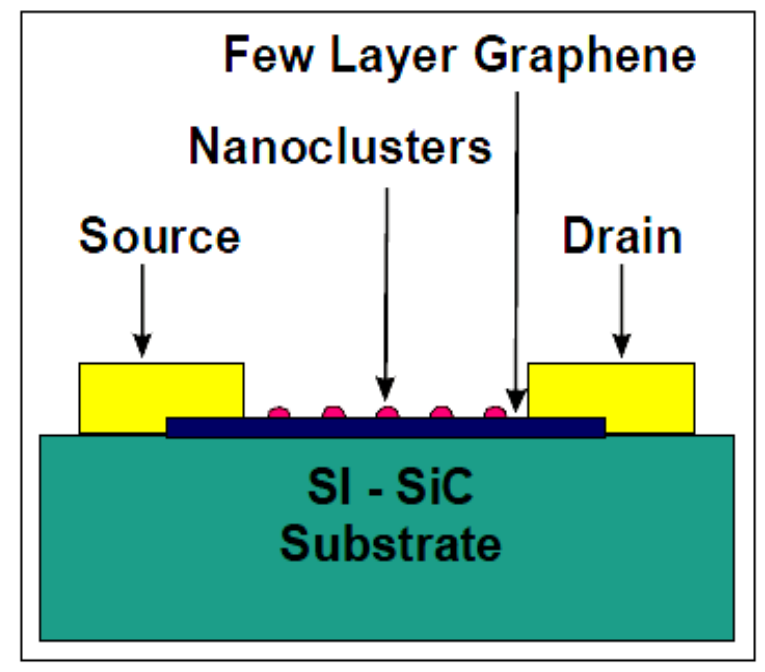

Figure 1.3: Schematic of graphene-based composite chemo resistive sensor illustrating a dispersed rather continuous layer of nanoparticles.

Within the laboratory, research has been divided into several broad areas. These include (a) optimization of the graphene synthesis process, (b) modification of the graphene surface using nanoparticles to provide a basis for sensor selectivity, (c) fabrication of basic device structures, (d) electrical characterization of the graphene and modified graphene, and (e) sensor characterization and optimization. The research described in this dissertation focuses on all areas but the last.

The optimization studies have been focused on the reliable and reproducible synthesis of single, bi and tri-layer graphene is synthesized on SiC substrates using inductively coupled plasma-reactive ion etching (ICP-RIE) and high temperature annealing. The effects of ICP-RIE parameters including RIE and ICP power, etch time, and bias voltage have been studied in detail. Similarly, annealing parameters like temperature, time, and environment (i.e., inert gas versus ultrahigh vacuum) were tested to improve quality of the graphene films.

In an effort to provide selective sensor response, the graphene surface has been modified. The primary method of surface modification has been the attachment (nucleation) of different types of nanoparticles on the graphene surface, however other chemical methods were explored as well. The nanoparticles of interest include metals such as silver $(\mathrm{Ag})$, gold $(\mathrm{Au})$, platinum (Pt) and Iridium (Ir). In these studies, a variety of solution-based chemistries were investigated. Because surface defects most likely serve as nucleation sites, the impact of graphene synthesis and device fabrication parameters which alter surface defect types and densities have been investigated. Specifically, reducing agents like 
hydrochloric acid and protic solvents like methanol were studied for their effect on defect type and density and their overall impact on nanoparticle nucleation.

Using these films, two primary device structures were formed. The first device was a simple wire-bondable contact array structure which allowed accurate measurements of electrical properties using two point or four point techniques. The second device was a wire-bondable sensor array structure which formed the basis of the chemo-resistive sensor. Here the emphasis was placed on wire-bondable structures, since this was required for the assembly of the sensor platform that is being used in gas response studies by other students and because the wire bonded contacts provide more reproducible measurements than the movable contact probes typically used in preliminary studies of electrical properties. Both structures were fabricated using photolithography and lithography-free methods for the deposition of source and drain contacts. Using the array structure, current-voltage (I-V) measurements were performed to study effect of film thickness on the electrical properties. Measurements of I-V characteristics are performed to determine the effect of nanoparticles on the electrical properties of graphene. As noted, the detailed characterization and optimization studies for the gas sensors are being performed by other students.

Thus, the specific objectives for this research are:

1) Optimize of etching and annealing process for reproducible production of mono, bi and trilayer graphene

2) Modify the surface by chemical reduction methods

3) Establish nanoparticle nucleation and growth on graphene surface for making composites

4) Deposition of metal contacts and electrical characterization of graphene and graphenenanoparticle composites 


\section{Chapter 2 BACKGROUND}

\subsection{Synthesis of Graphene}

\subsubsection{Chemical and Mechanical Exfoliation}

Two of the most common method used for graphene synthesis is chemical and mechanical exfoliation of highly ordered pyrolytic graphite (HOPG). In 2004, Researchers from Manchester University developed a technique to spilt graphene crystal into thinner pieces, resulting in the few-layer flakes attached to the adhesive scotch tape [1]. The tape can be etched away by acetone and those flakes can be dispersed onto a Si wafer. Although, high quality samples were obtained by mechanical exfoliation, the method has disadvantages, since it is time consuming and have low yield.

Chemical oxidation of graphite is well established using concentrated acids such as sulfuric acid and nitric acid with highly oxidizing agents (potassium permanganate). This method is widely known as Hummer's method [34]. Using this method, Stankovich et al. synthesized exfoliated graphene oxide and chemically reduced it using hydrazine [35]. According to them, the reduction starts from the edges of graphene oxide (GO) particles and proceeds into the basal planes. During the reduction, parts of the basal planes near the edges become reduced and subsequently snap together due to $\pi-\pi$ interactions, thus narrowing the interlayer distance. Consequently, the reducing agent, hydrazine, cannot penetrate further into the interior of the reduced graphene oxide (RGO) particles, presumably leading to the lower degree of reduction of RGO. Atomic force microscopy (AFM) showed $1 \mathrm{~nm}$ thickness, 3 times of that of pristine graphene, thus confirming tri-layer graphene films. As seen in Figure 2.1, change in peak intensity with XPS of GO flakes and reduced flakes with hydrazine confirms reduction of graphene oxide into $\mathrm{sp}^{2}$ bound carbon of the graphene flake.

High quality graphene was obtained using this method by transferring these flakes on to a substrate with thin $\mathrm{SiO}_{2}$ layer. Various reduction methods have been used since then to get better quality graphene. The problem is the flakes are rare and very difficult to find on the substrate surface and hence this method is not suitable for mass production of electronic devices. 


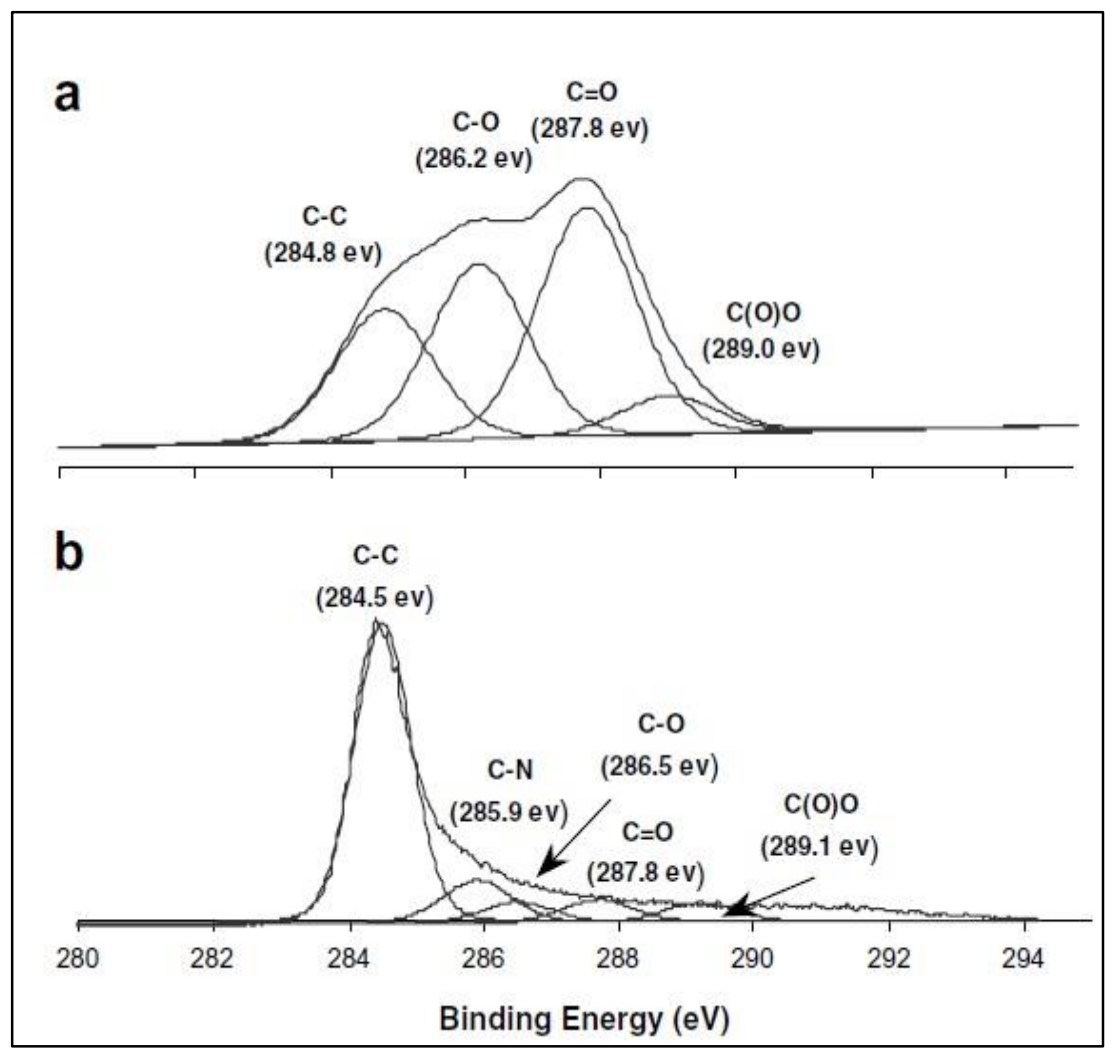

Figure 2.1: The C1s XPS spectra of: (a) GO, (b) reduced GO [35].

\subsubsection{Chemical Vapor Deposition(CVD)}

Chemical vapor deposition (CVD) on a metal substrates is another method that has been used widely for growing graphene. In CVD, carbon bearing species react at high temperatures in the presence of metal catalysts, serving in the decomposition of carbon species and in the nucleation of a graphene lattice. Using the material with low solubility of carbon in metal $(0.001 \%$ atomic), the synthesis of graphene is a process limited to the surface of the catalyst. Also, if the film is annealed at high temperatures, it experiences grain size growth needed for achievement of large uniform graphene domains. Thus, it gives flexibility in a foil of low thickness which can be used for deposition of graphene.

The synthesis of graphene on metal substrates by CVD was first reported in 2006 by Somani and coworkers [36]. In their work, camphor was evaporated into a chamber containing a nickel substrate held at $700-850^{\circ} \mathrm{C}$. This experiment demonstrated that graphene could be synthesized by a CVD process similar to that used for the synthesis of carbon nanotubes. 
A great interest in copper substrates has developed over the years since it was shown that single or bilayer graphene can be grown using this metal $[6,37,38]$. The low carbon solubility (<0.001 atomic \%) of copper at even high temperatures accounts for the self-terminating graphene monolayer growth.

Many carbon sources have been used to grow high quality graphene on copper foil but methane continues to be the carbon source of choice [39]. This growth takes place under high to moderate vacuum and at temperatures in the range of $1000-1035^{\circ} \mathrm{C}$ with hydrogen as the reducing gas. There are also some examples in the literature where graphene has been synthesized at lower temperatures $[38,40,41]$. Once graphene has been grown, the layer can be transferred to other substrates by chemical etching of the metal substrate.

In a paper by Bae et al, a roll-to-roll production of graphene films was reported [42]. The graphene films exhibited a sheet resistance of $30 \Omega$ /sq., a $90 \%$ optical transparency, and a relative high mobility of $5100 \mathrm{~cm}^{2} /$ V.s. These values a much less than those compared to those obtained by exfoliation method. Another major problem with CVD is that graphene is obtained on top of the metal surface, but for electronic devices applications, it needs to be transferred to insulating substrates e.g. $\mathrm{SiO}_{2}$. During the transfer process, unavoidable structural damage occurs to graphene which can degrade its electrical properties.

\subsubsection{Sublimation of Si from Silicon Carbide}

Van Bommel in 1975 first obtained monocrystalline graphite monolayer films layer grown on a hexagonal silicon carbide(SiC) in ultra-high vacuum (UHV) at temperatures above $800^{\circ} \mathrm{C}$ [43]. Silicon sublimation from the SiC causes a carbon rich surface that nucleates an epitaxial graphene layer. The graphene growth rate was found to depend on the specific polar SiC crystal face. Graphene forms much slower on the silicon-terminated face (0001) surface (or Si-face) than on the carbon-terminated face (0001) surface (or C-face). He also identified these films that can be decoupled from the SiC substrate and therefore were electronically equivalent to isolated graphene sheets. Since 1975, these films were referred to as monolayer graphite, or two-dimensional graphite crystals or epitaxial graphene.

Using grazing-incidence $x$-ray diffraction and scanning tunneling microscopy(STM),Charrier demonstrated that the thermal decomposition of an electronic-grade wafer of $6 \mathrm{H}-\mathrm{SiC}$ after annealing at increasing temperatures between 1080 and $1320^{\circ} \mathrm{C}$ leads to the layer-by-layer growth of unconstrained, heteroepitaxial single-crystalline graphite [44]. 
Berger and coworkers produced high quality graphene sheets by heating them to temperature as high at $1250^{\circ} \mathrm{C}$ to $1450^{\circ} \mathrm{C}$ [45]. They also demonstrated that thinner films were produced using $\mathrm{Si}$ terminated surface (1-5 layers) as compared to C terminated surface (4-100 layers). In general, they showed that the number of layers increased with increasing temperature.

\subsubsection{Reactive lon Etching}

Raghavan et al. studied graphene synthesis by using inductively coupled plasma- reactive ion etching (ICP-RIE) with the halogen based gases like $\mathrm{Cl}_{2}$ and $\mathrm{CF}_{4}$ [46]. Silicon was selectively removed from $6 \mathrm{H}-\mathrm{SiC}$ substrate using halogen gases to produce a carbon rich layer on the substrate. These etched samples were annealed in UHV chamber at temperatures as high as $970^{\circ} \mathrm{C}$. The carbon rich layer undergoes reconstruction to produce one to three graphene on SiC surface. Various samples were synthesized using this method and characterized to determine its quality.

Figure 2.2(a) shows the C1s x-ray photoelectron spectroscopy (XPS) for a nominally two layer graphene film prepared by $\mathrm{CF}_{4}$ based plasma. The photoelectron peak at $282.5 \mathrm{eV}$ corresponds to carbon in the SiC surface and the peak at $284.1 \mathrm{eV}$ corresponds to $\mathrm{sp}^{2}-\mathrm{C}$ in the graphene film. The two higher binding energy peaks are due to the fluorine defects as shown in Figure 2.2(b), described in detail by Sato and coworkers [47]. This defect consists of two coupled $\mathrm{sp}^{3}-\mathrm{C}$ atoms and each bound to an F-atom with one F-atom above the surface and one below the surface. These carbon atoms are referred to as $\mathrm{C}_{\mathrm{F}}$ species in the inset and they correspond to the peak at $285.7 \mathrm{eV}$. The two $\mathrm{sp}^{2}-\mathrm{C}$ atoms bound to each of the $\mathrm{C}_{\mathrm{F}}$ species correspond to the peak at $288.4 \mathrm{eV}$ and referred to as $\mathrm{C}_{\mathrm{C}}$ species since they are bound to other Catoms in the graphene film.

These defects actually cause buckling of the graphene films and will be referred as "buckled graphene". These defects are of importance as they can serve as target sites for incorporating reactivity into the relatively inert graphene sheets, making them amenable to facile tethering and attachment to other functionalities. The concentration of the defect species can be controlled by the annealing temperature and will be useful in controlling the density of nanoparticle nucleation and growth sites. 


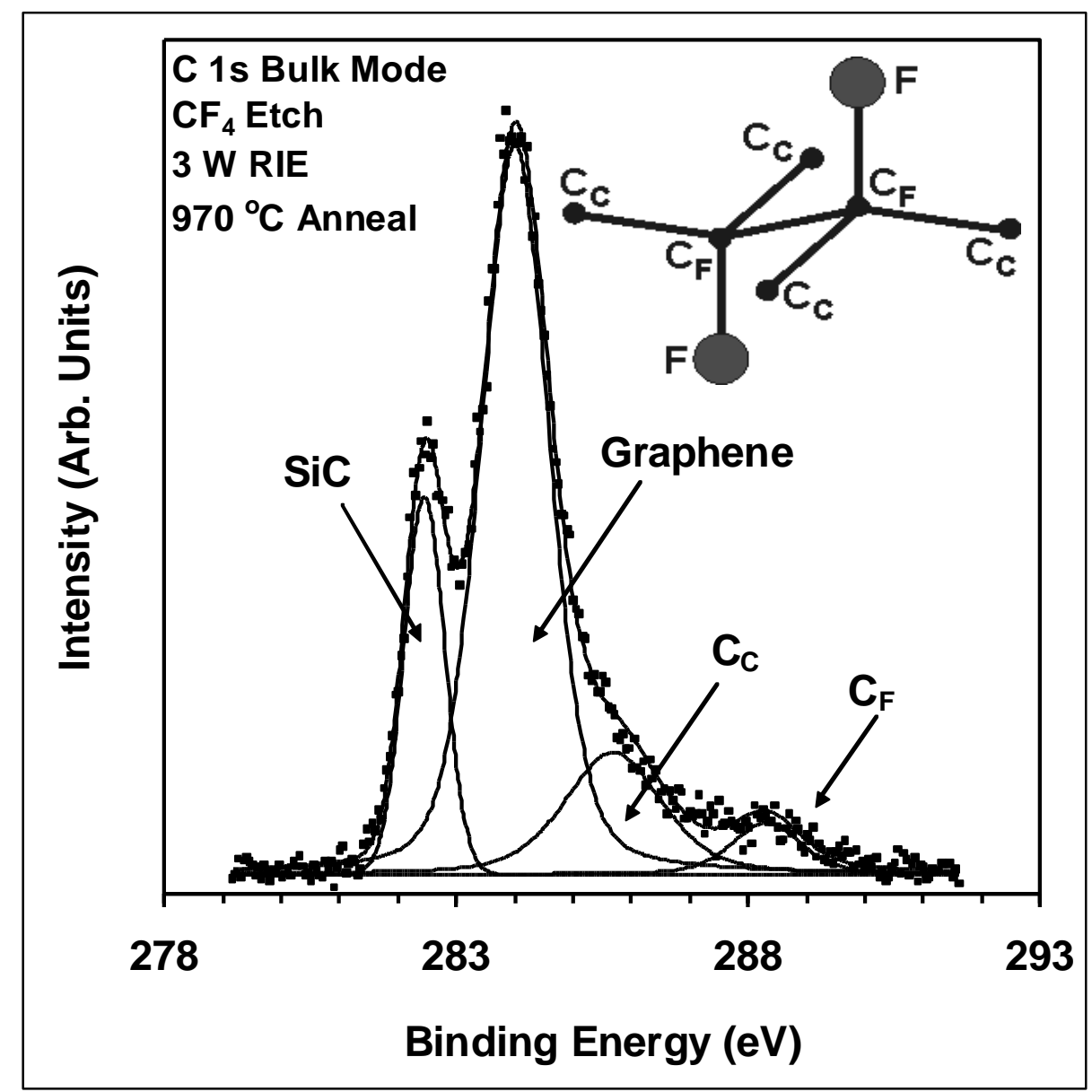

Figure 2.2: $C$ 1s XPS spectrum for graphene film. The inset shows $C_{C}$ and $C_{F}$ defects associated with the peaks [46].

The presence of graphene was further verified by characterization methods like Raman spectroscopy and reflection high energy electron diffraction (RHEED). Figure 2.3(a) shows two Raman bands, D (1350 $\left.\mathrm{cm}^{-1}\right)$ and $\mathrm{G}\left(1583 \mathrm{~cm}^{-1}\right)$ representative of the $6 \mathrm{H}-\mathrm{SiC}$ surface (i) after $\mathrm{HF}$ dip and (ii) after $\mathrm{CF}_{4}$ based ICPRIE and annealing. Figure 2.3(b) shows the corresponding 2D $\left(2691 \mathrm{~cm}^{-1}\right)$ Raman bands. The intensity of these peaks for the etched and annealed surface is in good agreement with two layer graphene films prepared by Ley and coworkers. The relatively low intensity of the D band is due to low level of edges.

The Figure 2.3(c) shows the RHEED pattern obtained with the direction of the beam along the $[1 \overline{1} 00]$ axis of the $6 \mathrm{H}-\mathrm{SiC}$ surface. The lower pattern corresponds to the SiC (0001) surface after an $\mathrm{HF}$ acid dip to remove oxide, while the upper pattern corresponds to surface after $\mathrm{CF}_{4}$ based ICP-RIE and annealing at $970^{\circ} \mathrm{C}$. The two faint spots seen in the upper pattern are due to the grapheme over layer, which are consistent with the RHEED patterns obtained by Moreau [48]. 


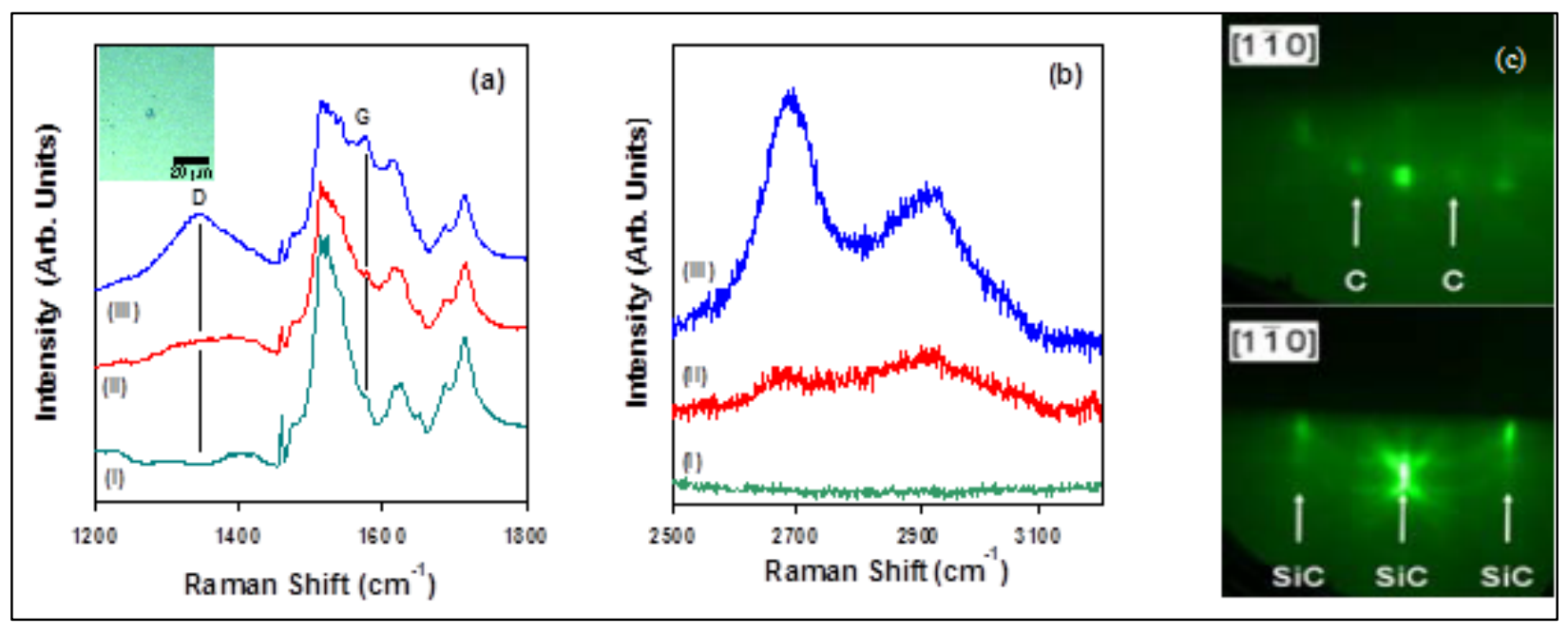

Figure 2.2: (a) The $D$ and $\mathrm{G}$ spectral region for (i) the $6 \mathrm{H}$-SiC substrate ii) a two layer graphene film iii) a pit like defect in two layer film with the optical micrograph inset. (b) The corresponding $2 D$ spectral region(c) RHEED pattern [46].

\subsection{Graphene as Solid State Sensor}

Solid state gas sensors are known for their high sensitivity, low cost and small size. As the level of sensitivity should be as small as $1 \mathrm{ppb}$, sensors with high sensitivity are desired for industrial applications. One of the major drawbacks of the current sensors is fluctuations in properties by thermal motion of charge carriers and defects. These fluctuations create electrical noise which limits sensitivity. In addition, even for active sensor films a micron or so thick, only a small percentage of the charge carriers available are involved in the detection process. Another figure of merit for sensors is response time. For solid state sensors response times on the order of tens to hundreds of milliseconds are possible [49]. Although this is rapid relative to other gas detection methodologies, improvements are desirable.

The carbon nanotube-based gas sensors evolved as a promising candidate as a solid state gas sensor due to their superior electronic and mechanical properties. In spite of that, it's one dimensional structure is not suitable for use with the existing standard electronic device fabrication technologies. Here the properties of graphene can be advantageous. Since graphene is essentially all surface, a high percentage of the carriers are available to participate in the gas adsorption process. This should significantly enhance the sensitivity of graphene compared to other solid state sensors. As noted earlier, Novoselov claims that this has allowed detection of single molecule adsorption on graphene platform under certain conditions [25]. The high mobility of the charge carriers should contribute to rapid response times for these graphene based sensors. At the same time, the chance of getting low frequency noise is more likely with high sensitivity. But because of its two dimensional nature and widely tunable carrier concentration of 
graphene as it is possible to detect changes in carrier concentration by a few electrons with less noise and the response can be measured easily by four point probe technique, making it a perfect future material for sensor applications.

Schedin was the first who demonstrated the detection of gaseous species like $\mathrm{NO}_{2}, \mathrm{NH}_{3}$ and $\mathrm{H}_{2} \mathrm{O}$ on graphene by measuring changes in resistivity due to adsorption of these gases [25]. He found that these gases act as either donors or receptors, thus changing the charge carrier concentration. Graphene sensors were found to be electronically stable in external forces like mechanical strain and magnetic field. These observations lead to the idea of graphene as one of the future promising sensor materials. Graphene based resistive gas sensors are commonly reported in the literature since then.

In resistive gas sensors, the changes in the resistance between the source and drain are observed due to the adsorption of the target species. It is most widely used in research because of its relatively easy fabrication and operation. The resistance of the film across the electrodes is measured either directly with a multimeter or from the current-voltage (I-V) characteristics. There are four major parameters which affect sensor performance: the effect of substrates, the effect of layers, the effect of surface functionalization or composites and the effect of source-drain electrodes.

As graphene is a one atom-thick layer of carbon atoms, it has to be deposited on different substrates before being used for sensor applications. Thus the choice of different substrates also plays a role in manipulating the properties of graphene for sensing applications. The commonly used substrates for graphene-based gas sensors are: silicon wafer $\left(\mathrm{SiO}_{2} / \mathrm{Si}\right)$, silicon carbide $(\mathrm{SiC})$, soda-lime glass, and silicon nitride $\left(\mathrm{SiN}_{\mathrm{x}} / \mathrm{Si}\right)$. Under similar conditions, for a given target gas, the sensitivities have been reported in the order: $\mathrm{SiC}>\mathrm{SiN}_{x} / \mathrm{Si}>\mathrm{SiO}_{2} / \mathrm{Si}>$ Soda-lime Glass [50]. The effect of substrates on sensing behavior was reported to be more pronounced when field effect transistors were used for sensing purposes.

Pearce showed that when graphene is synthesized on SiC substrates, electrons are donated to the graphene films making it $n$-type with very low carrier concentration [49]. One of the advantage with graphene is low free carrier concentrations, thus successive adsorption of target species such as $\mathrm{NO}_{2}$ due to the long time and high concentration exposure cause the transition of carrier type from $n$-type to $p$ type and electronic properties of graphene can be tuned by the transfer of electrons from substrates. This property can be utilized to detect very low concentration of target gas. For the graphene synthesized using CVD, it has to be transferred to different substrates. The sensitivity for certain gases such as hydrogen was reported to be higher from graphene on a silicon wafer than from graphene on SiNx/Si. Although the exact reason is not clear, a possible $\mathrm{C}-\mathrm{O}$ bonding between the graphene and the thermally oxidized $\mathrm{SiO}_{2}$ seems to increase the bonding strength of graphene. This increase in bonding strength between the 
graphene and the substrate degrades the carrier transfer properties in comparison with a $\mathrm{SiN}_{\mathrm{x}}$ gate insulator [51].

The multi-layer graphene sensor device was reported to have lower sensitivity as compared to the single layer graphene as the charge conduction was not due to the gas exposed to the uppermost layer alone. The large number of charge carriers in the uppermost graphene layer as a result of the process of adsorption of target species is screened by the substrate and hole-doping by the atmospheric oxygen.

The enhancement in the sensing behavior of graphene by the process of surface functionalization was also reported by many researchers $[52,53,54,55]$. To explore the influence of defects on the sensing performance of graphene, Masel and co-workers used pristine graphene and polycrystalline graphene with wrinkles [56].They found that the sensitivity of a graphene-based sensor depends on the type and geometry of graphene defects for determining vapors of organic compounds. Pristine graphene with few point defects is insensitive to vapors of 1, 2-dicholorbenzene and toluene. However, the sensing response was greatly enhanced by introducing defects to the graphene sheet because of reduction of conduction paths around such defects. In order to improve sensing behavior, the sheets were cut into ribbons with widths in the range of 2 to 5 micron and the sensing response was increased by 2 times over that of an unpatterned CVD graphene, as seen in Figure 2.4.
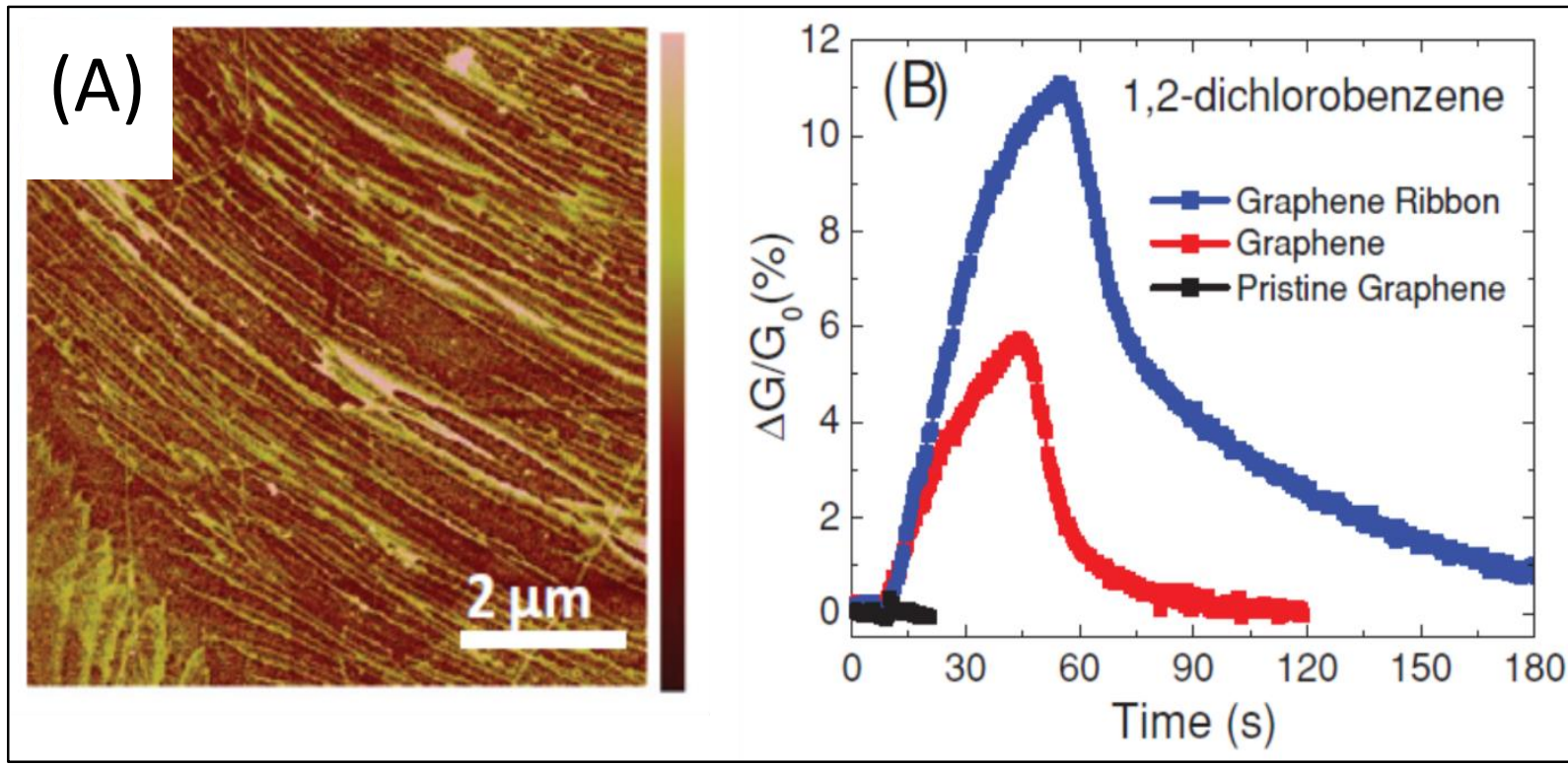

Figure 2.3: (a) AFM images of CVD-graphene used for sensors. (b) Response of the defective CVDgraphene, CVD-graphene micro ribbon and $5 \mathrm{~mm}$ wide exfoliated graphene-based sensors to $10^{15}$ molecules of 1, 2-dichlorobenzene [56]. 
As the graphene with defects was seen to have better sensitivity, different treatments were studied to introduce defects into pristine graphene for better performance. Ozone treatment was found to be one of the most effective technique to induce an oxygen-containing groups in graphene. The advantage of the ozone treatment is that it induces uniform distribution of oxygen groups on the edge as well as the whole basal plane of the graphene sheet effectively [57]. The gas sensors based on the ozone-treatment demonstrated significant enhancement in sensing performance, as shown in Figure 2.5. The percentage response to $200 \mathrm{ppm} \mathrm{NO}$ was 2 times stronger and the response time was eightfold faster than those of a pristine graphene sensor. Also, the low limit of detection to $1 \mathrm{ppb}$ was achieved with these graphene samples.

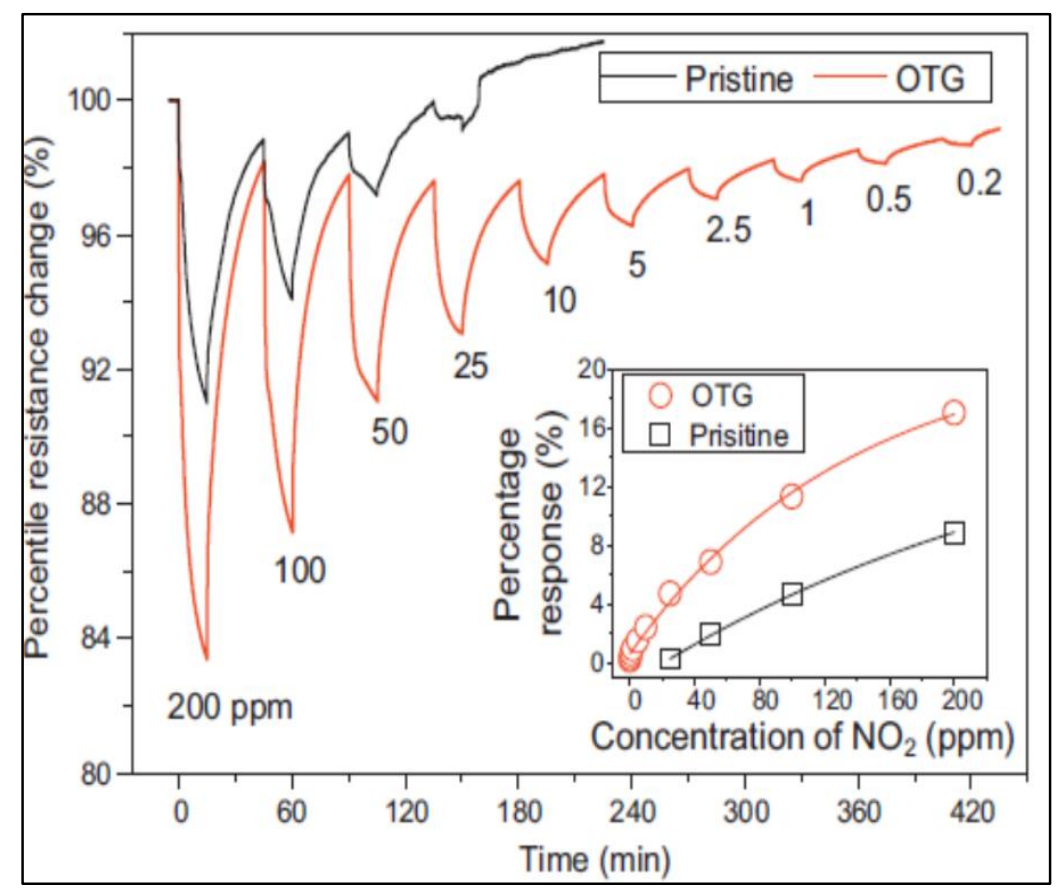

Figure 2.4: The percentile resistance changes of the Ozone treated graphene (OTG) (red) and pristine graphene (black) sensors. The $\mathrm{NO}_{2}$ concentration was modulated from $200 \mathrm{ppm}$ to $200 \mathrm{ppb}$. The inset shows the correlation between percentage response and concentrations, which is in agreement with the langmuir adsorption model [57].

As the graphene oxide and reduced graphene oxides are heavily oxygenated by hydroxyl and epoxy group on $\mathrm{sp}^{3}$ hybridized carbon on the basal plane and carbonyl and carboxyl group on $\mathrm{sp}^{2}$ hybridized carbon at the sheet edges, it is promising material for the fabrication of gas sensors with practical application. They can also be prepared at relatively low cost and on a large scale, providing an advantage over expensive synthesis methods. The functional groups provide reactive sites for further functionalization and gas adsorption. The only problem associated with these kind of defects is low 
conductivity, which makes it not suitable for practical application. But by controlling these defects by chemical or thermal reduction, the conductivity can be restored with some amount of oxygen defects still present. The reduction process can also introduce some vacancies and structural defects which can also act as adsorption sites [58]. It was also demonstrated that the interaction of gas molecules with high energy defects differs than that with conjugated carbon structures. The defect adsorption dominates the electrical response of the graphene but desorption from the defect was much slower than that from pristine graphene [59]. Therefore, optimization of defect density is an effective route to balance sensitivity and the recovery rate of a reduced graphene oxide (RGO) based gas sensor.

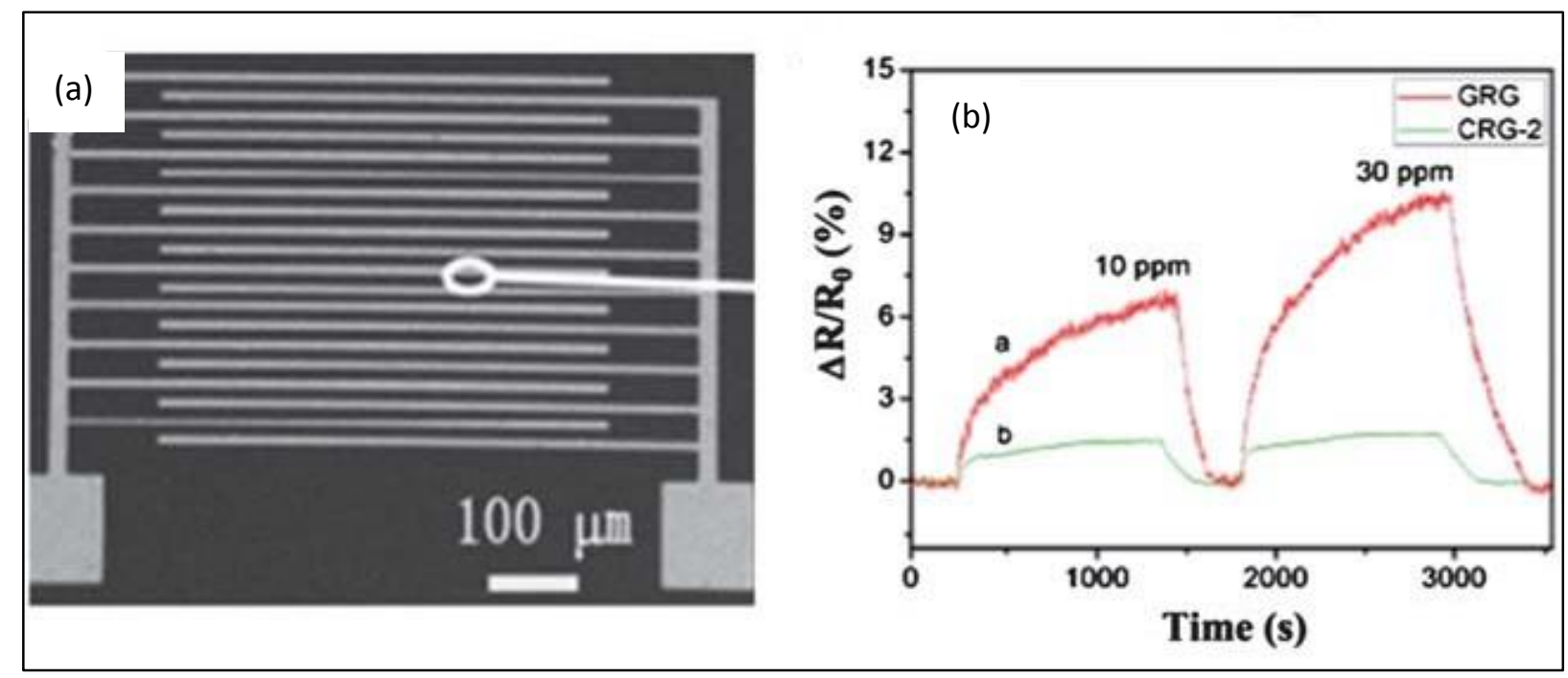

Figure 2.5: (a) SEM image of RGO membrane deposited between electrode arrays. (b) The comparison of the resistance changes between chemically reduced graphene (CRG) (reduced from $p$ phenylenediamine) sensor and CRG-2 (reduced from hydrazine) sensor at different co concentrations of dimethyl methyl phosphonate [60].

Zhang and co-workers developed a gas sensor based on p-phenylenediamine reduced GO [60]. A drop drying method was employed to create a conductive network of RGO sheets between interdigited electrode arrays. The sensor based on this procedure exhibited 3.3-4.7 times stronger response to 10-30 ppm dimethyl methyl phosphonate compared to that of hydrazine reduced GO, as seen in Figure 2.7. Furthermore, its response repeatability was much better as more oxygenated groups and structural defects are present due to the weak reducing activity of $p$-phenyldiamine.

In similar studies, another weak reducing agent, ascorbic acid was used for reducing the RGO film on flexible substrates [61]. The resulting film reversibly and selectively detects chemically aggressive vapors such as $\mathrm{NO}_{2}, \mathrm{Cl}_{2}$ with the limit of detection in the range of $500 \mathrm{ppb}$ to $100 \mathrm{ppm}$.

Yuan reported chemiresistor-type $\mathrm{NO}_{2}$ sensors based on chemically modified graphene including sulfonated RGO(S-G) and ehtylenediamine-modified RGO (EDA-G) [62]. These sensors showed 4 to 16 time 
higher response towards $\mathrm{NO}_{2}$ than that of the $\mathrm{RGO}$ counterpart. The electron-deficient $\mathrm{NO}_{2}$ molecules are prone to be adsorbed onto the lone-pair electrons in those functional groups. On the basis of this unique sensing mechanism, the CCG-based sensor has relatively good selectivity to $\mathrm{NO}_{2}$. As can be seen in Figure 2.7(b), upon exposure to $50 \mathrm{ppm}$ toluene or water vapor, no detectable conductance change can be observed. Since the electrostatic interaction is weak, $\mathrm{NO}_{2}$ molecules can be easily desorbed from the sensing materials by flowing $\mathrm{N}_{2}$, and thus achieving good reversibility (Figure 2.7(c)). The thickness of the sensing layer also has a strong influence on the sensitivity of the sensors. The thinner the sensing layer the higher the sensitivity of the sensor. A dip coating method has been employed to obtain an ultrathin

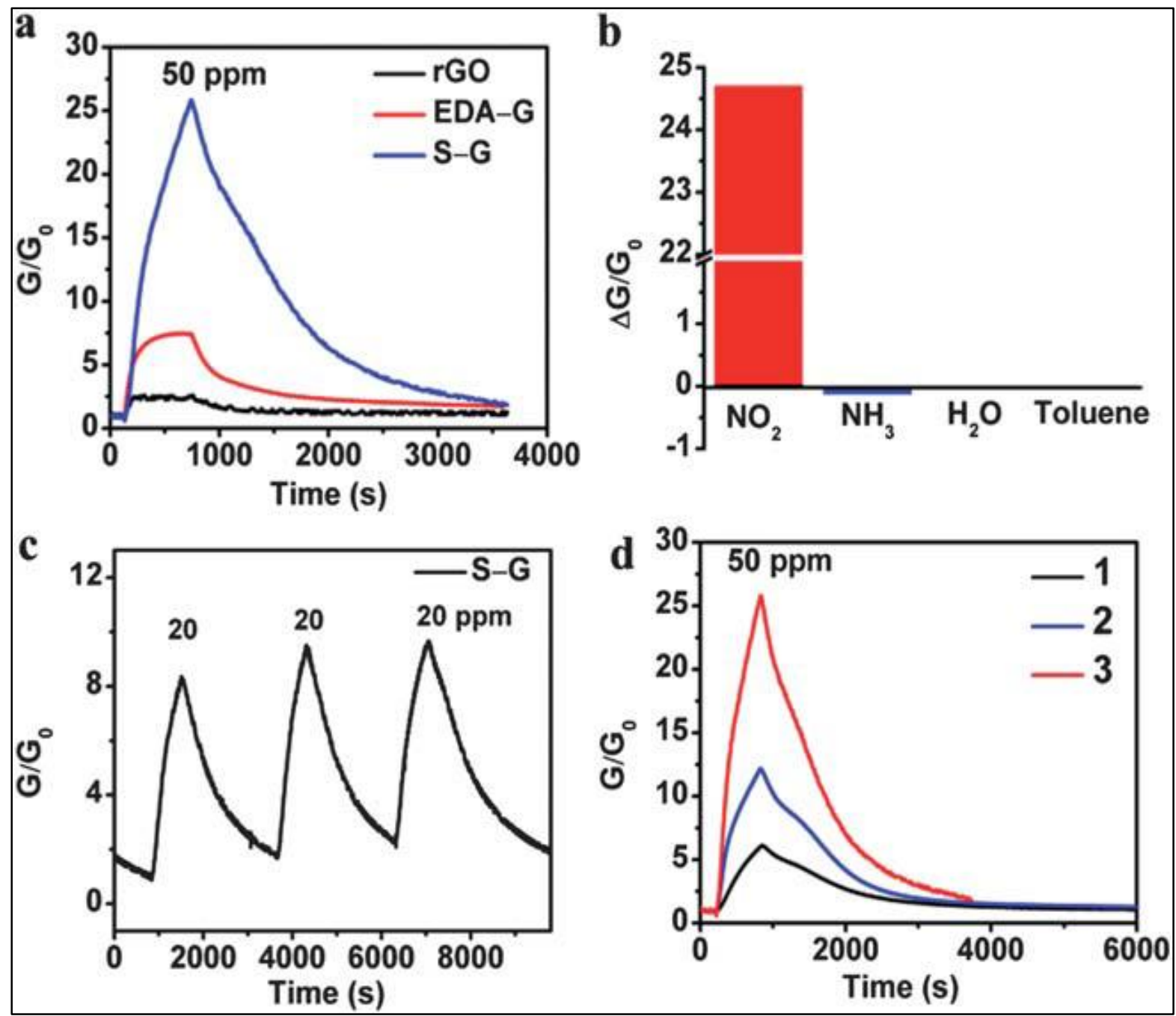

Figure 2.6: (a) Comparison of the responses of RGO-, Sulfonated $G O(S-G)$, and ethylenediamine-

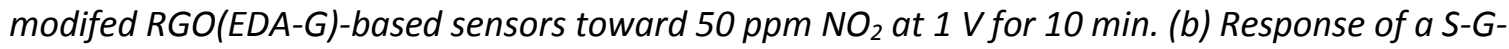
based sensor to $50 \mathrm{ppm} \mathrm{NO}, \mathrm{NH}_{3}, \mathrm{H}_{2} \mathrm{O}$ or toluene. (c) Conductance changes of an $\mathrm{S}-\mathrm{G}$ based sensor during 3 success of exposing to 20 ppm NO $\mathrm{N}_{2}$ for $10 \mathrm{~min}$ and $\mathrm{N}_{2}$ flow for $30 \mathrm{~min}$. (d) Responses of the S-G-based sensors with different thicknesses of sensing layers after exposure to $50 \mathrm{ppm} \mathrm{NO}$ : $50 \mathrm{~nm}$ (1), $10 \mathrm{~nm}$ (2), and a few layers (1-6 nm) of S-G sheets (3) [62]. 
graphene membrane of 1-6 $\mathrm{nm}$ and the resulting sensor exhibited much higher sensitivity compared with those of the devices with thicker sensing layers (10 to $50 \mathrm{~nm}$ ), as shown in Figure 2.7(d).

Actually, sensitivity is one of the most important parameters for evaluating the performances of gas sensors. Except for thinning the sensing layer and chemical modification, the use of graphene composites to replace pure graphene has also been attempted for improving the sensitivities of graphene-based gas sensors.

\subsection{Nanoparticle Nucleation}

Since, the synthesis studies established growth of graphene with reproducible results and desired defects, the next step is to functionalize them for applications, especially in the field of sensors. Adsorption mediated by different nanoparticles attached to independent graphene chemo-resistive sensors can yield an electrical response pattern specific to each species and this approach can make sensors suitable for a wide range of environmental conditions. The use of nanoparticles like Pt and $\mathrm{Au}$ supported on materials like carbon nanotubes is widely employed for catalytic purposes [63].Design and synthesis of materials with tailored size and shape is a fundamental goal of materials science because it is well recognized that properties of nanoparticles like durability and distribution of particles are significantly dependent on their composition, size, shape, structure, and crystallinity. Hence interfacial interaction between them needs to be studied for better performance and economic viability $[64,65,66$, 67]. The solution based approaches (colloid-chemical methods) of nonspherical noble metal nanoparticles principally involve the reduction of metal salts or the decomposition of metallic compounds in aqueous or organic solvents in the presence of a stabilizer and shape-control additives such as surfactant, ligand, polymer, and foreign ion [68, 69, 70, 71].

For the synthesis of a graphene nanostructures ( $\mathrm{Ag} \mathrm{NP}), \mathrm{AgNO}_{3}$ is the common precursor, which can be easily reduced in the presence of different reducing agents including amines, $\mathrm{NaBH}_{4}$ and ascorbic acid [72]. Dutta et al. reported synthesis of Ag-nanoparticle conjugated RGO nanosheets using dimethylformamide (DMF) as an efficient reducing agent that reduces both silver nitrate $\left(\mathrm{AgNO}_{3}\right)$ and graphene oxide (GO) in the reaction mixture [67]. Solomon et al. used $\mathrm{NaBH}_{4}$ along with $\mathrm{AgNO}_{3}$ for production of silver nanoparticles and was able to produce nanoparticles of 10-12 nm [73].

Some approaches of synthesis of gold nanoparticles (Au NP) include the Turkevich method, the Brust method, the Martin method, and the seed-growth method. The Turkevich method was pioneered by Turkevich and his coworkers in 1951 and refined by Frens in 1973 [74, 75].It simply adds chlorauric acid 
into boiled water, and then adds a small amount of sodium citrate solution to reduce the gold ion and produce monodispersed spherical gold with size around $15 \mathrm{~nm}$.

Gold nanoparticles on graphene are important for various biological and gas sensor applications. Goncalves et al. examined the role of oxygen moieties at GO and RGO surfaces on the gold nucleation and growth [76]. They reported that the nucleation and growth mechanism depends strongly on the degree of oxygen functionalization on the graphene surface. No Au nanoparticles were observed at totally reduced graphene surfaces. Fabrication of microporous gold films using graphene sheets as template was reported by Sun and Wu [33]. Gold nanoparticles decorated graphene sheets were prepared using a onepot simultaneous reduction of $\mathrm{GO}$ and gold precursor $\mathrm{HAuCl}_{4}$ by sodium citrate. Koo et al. observed the growth of sub-nano sized Au clusters on r-GOs, which could reinforce the conductivity of the resulting $r$ GOs by defect filling [77]. The resulting Au/r-GOs were reported to exhibit an improvement of bulk electrical conductivities and a reduced ratio of the intensity of the $D$ band to that of the $G$ band $\left(I_{D} / I_{G}\right)$, relative to the RGOs without Au nanoclusters. Solomon, in their nanoparticle studies, found that $\mathrm{NaBH}_{4}$ reduction with $\mathrm{HAuCl}_{4}$ produces gold nanoparticles similar to silver by a single step synthesis [73].

Pt nanoparticles(Pt NP) supported on a highly dispersed support are one of the most widely used catalyst materials for many chemical reactions as well as energy conversion devices including hydrogen oxidation and oxygen reduction reactions in fuel cell [63]. Thus, significant efforts have been focused on combining graphene as a conductive support for Pt nanostructures. There are couple of advantages with graphene as support. The surface area of graphene is exceptionally high and the potential to reduce carbon corrosion makes it an ideal material in enhanced electro-catalytic performance and long term stability.

Most of the techniques that were used to prepare graphene-Au or Ag composites and discussed above could be equally applied in general to fabricate graphene supported Pt nanomaterials. As a pure metal, bulk platinum does not oxidize at any temperature and has an excellent resistance to corrosion. This property allows platinum to be widely employed as a noble metal electrode in electrochemistry. The other most important application for platinum is as a catalyst in a number of separate processes, especially in the automobile industry. Platinum powder allows complete combustion of unburned hydrocarbons from the exhaust, and converts them into harmless carbon dioxide and water.

Many efforts have been involved to make various shapes of platinum nanoparticles in order to investigate their influence on catalytic activity. On this point, uniformly dispersed platinum nanoparticles are needed to quantify the catalytic influences. Similar with the morphology control of other noble metal nanoparticles, capping agents such as $\mathrm{CTAB}, \mathrm{PVP}, \mathrm{KBr}, \mathrm{KCl}, \mathrm{AgNO}_{3}$ are usually used to favor the growth of 
particular facets. Ahmadi and co-workers reported size and shape control of platinum nanoparticles by changing the ratio of the capping polymer (sodium polyacrylate) to the concentration of the metal salt [78]. Yang et al. also demonstrated that cuboctahedrons, cubes, and porous platinum nanoparticles can be obtained by adjusting the reduction method, and the silver ion plays a significant role in morphology control for platinum nanoparticles [79].

Iridium (Ir) is corrosion-resistive and exhibits high stability even in acidic solutions [80]. Work by Goldstein has shown that a thin coatings of Ir can extend the range of operating conditions for graphite to $2110^{\circ} \mathrm{C}$ [81]. As a ruggedization measure for operating sensors at high temperature, it is desired to study the interaction of the Ir nanoparticles and/or thin films with the graphene surface. In fact, very few research groups have studied the interaction of Ir with the graphene film. 


\section{Chapter 3 EXPERIMENTAL METHODS}

The experiments performed in this dissertation utilized the shared research facilities (SRF) cleanroom and surface and material studies (SMS) lab. In these studies, different SiC substrates were degreased and then plasma-etched in ICP-RIE chamber using $\mathrm{CF}_{4}$ gas. These samples were annealed under various annealing environments, temperatures and time. Photolithography and ICP-RIE were used to pattern the surface. E-beam evaporation deposited metal electrical contacts. Nanoparticles were deposited using colloids of different metals. Samples were analyzed using XPS, reflective high energy electron diffraction (RHEED), Raman spectroscopy and AFM, while semiconductor parameter measurements were made to characterize the electrical properties. A brief description of these process \& measurement is given below.

\subsection{Fabrication}

\subsubsection{Substrate and Sample Preparation}

Silicon carbide occurs in many different crystal structures, called polytypes. Despite the fact that all SiC polytypes chemically consist of $50 \%$ carbon atoms covalently bonded with $50 \%$ silicon atoms, they have different electrical and semiconductor properties. The most common polytypes of SiC presently being developed for electronics are the $4 \mathrm{H}-\mathrm{SiC}$ and $6 \mathrm{H}-\mathrm{SiC}$. The different polytypes of $\mathrm{SiC}$ are actually composed of different stacking sequences of $\mathrm{Si}-\mathrm{C}$ bilayers, also called $\mathrm{Si}-\mathrm{C}$ double layers. Each atom within a bilayer has three covalent chemical bonds with other atoms in the same (its own) bilayer, and only one bond to an atom in an adjacent bilayer. The stacking sequence of the $4 \mathrm{H}-\mathrm{SiC}$ polytype requires four $\mathrm{Si}-\mathrm{C}$ bilayers to define the unit cell repeat distance along the c-axis or stacking direction Similarly, the $6 \mathrm{H}-\mathrm{SiC}$ polytype repeats its stacking sequence every six bilayers throughout the crystal along the stacking direction, as shown in Figure 3.1 [82]. In the C-axis direction, SiC is a polar semiconductor [83]. One surface (0001) is normal to the c-axis is terminated with silicon atoms while the opposite surface (000 $\overline{1})$ is terminated with carbon atoms. These surfaces are typically referred to as "the silicon face" and "the carbon face" surfaces.

The samples used in these studies were $1 \mathrm{~cm} \times 1 \mathrm{~cm}$ squares diced from chemo-mechanically polished on axis $\left( \pm 0.5^{\circ}\right) 6 \mathrm{H}$-SiC (0001) n-doped wafers purchased from CREE. The nominal resistivity of these wafers was around $0.020-0.200 \Omega-\mathrm{cm}$. The nominal surface roughness for these wafers was less than $5 \mathrm{~nm}$ RMS. In all reported cases, films were formed on the Si-surface. Comparable wafers from other 


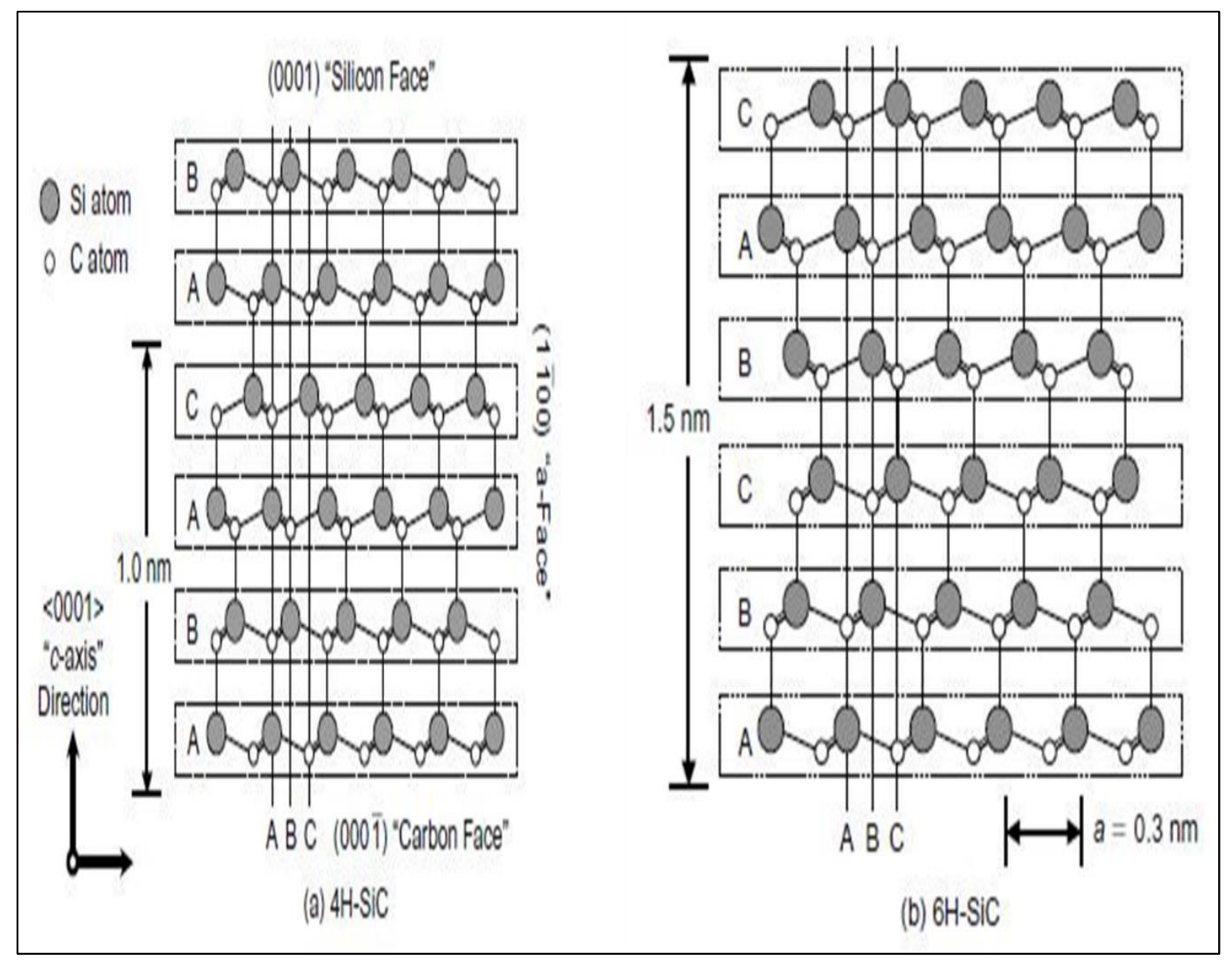

Figure 3.1: Structure of different SiC polytypes (a) 4H-SiC (b) 6H-SiC [82].

vendors (e.g., University Wafer, Inc.) were also used. Prior to use, the samples were degreased using trichloroethylene, acetone, and methanol. This was followed by blow drying in ultrahigh purity $\mathrm{N}_{2}$.

Figure 3.2 shows the $\mathrm{C} 1 \mathrm{~s}$ spectra for the (0001) $6 \mathrm{H}-\mathrm{SiC}$ surface after the initial cleaning to remove most of the particulates. The peak at $282.5 \mathrm{eV}$ corresponds to the carbon bond to silicon in bulk SiC [84]. The peak at $284.8 \mathrm{eV}$ corresponds to $\mathrm{C}-\mathrm{C}$ bonds and often associated with a fractional monolayer of graphite. The low intensity peaks at $286.2 \mathrm{eV}$ and $287.9 \mathrm{eV}$ are associated with $\mathrm{C}-\mathrm{O}$ and $\mathrm{C}=\mathrm{O}$ bonds due to oxygen contamination due to exposure to air.

These wafers are transparent in nature, hence marks were made onto the $C$ terminated sides to identify difference between Si terminated and C terminated surface. These wafers were diced by American Precision Dicing (and later using the SRF dicing saw) into $1 \mathrm{~cm} \times 1 \mathrm{~cm}$ samples and degreased with acetone and methanol, prior to their use. 


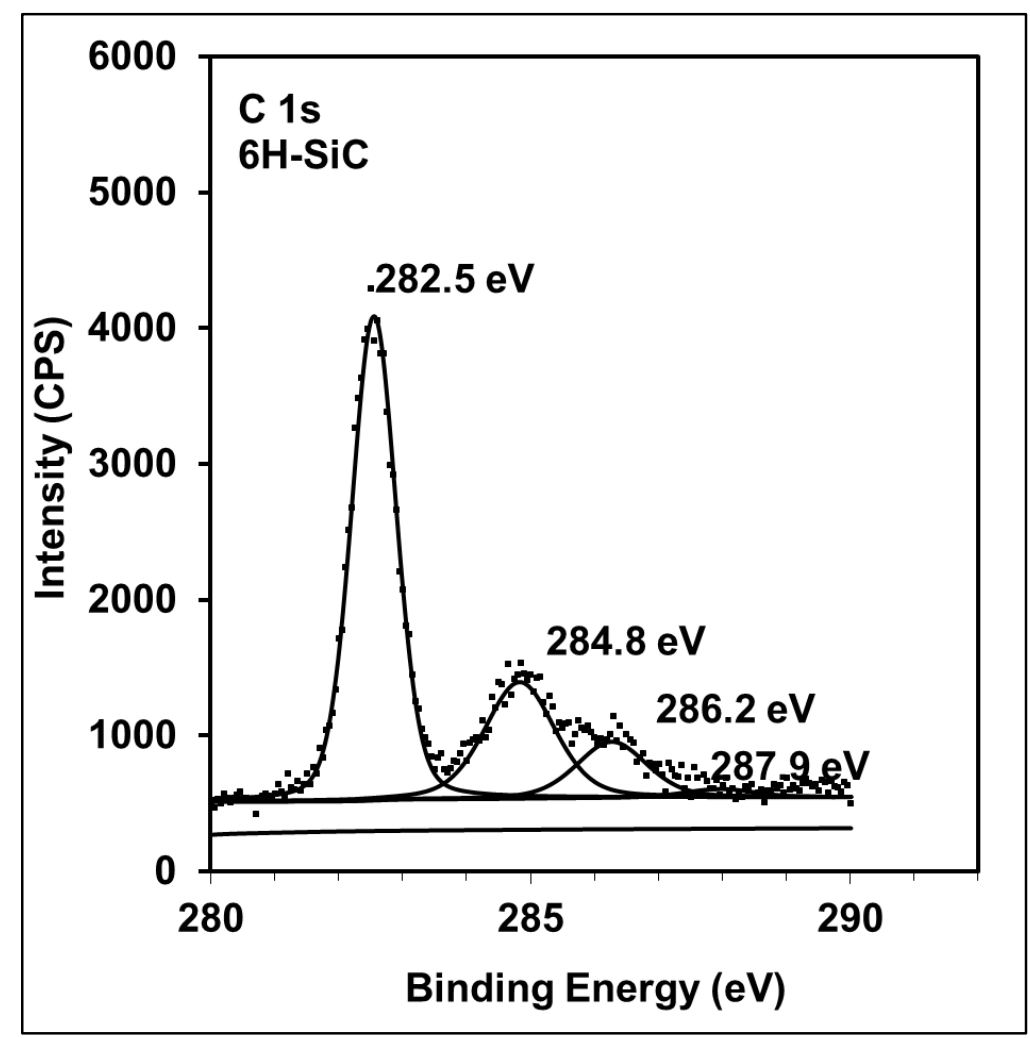

Figure 3.2: C1s XPS spectra for 6H-SiC (0001) surface after degreasing.

\subsubsection{Inductively Coupled Plasma-Reactive Ion Etching (ICP-RIE) System}

Plasma etching may be categorized into two different subsets: physical etching and chemical etching. In chemical etching, material is removed due to chemical reactions with the material that form volatile compounds. In physical etching, material is removed due to momentum and energy transfer. In addition, when etching a compound such as $\mathrm{SiC}$, the question of etch selectivity arises. Chemical etching can be very selective because etch rates on different element are strongly dependent on the chemical reaction effects. In contrast, physical etching is much less selective to elements of similar mass.

Plasma etching techniques are used for making integrated circuits by etching semiconductors like $\mathrm{Si}$, SiC and GaN. The ICP-RIE system used in present work is the Minilock-Phantom iii from Trion technologies [85]. This is located in the SRF cleanroom in the class 1000 portion and as shown in Figure 3.3(a).This system operates at $13.56 \mathrm{MHz}$ to provide up to $1250 \mathrm{~W} \mathrm{ICP}$ power and $600 \mathrm{~W}$ RIE power and has a parallel-plate electrode configuration as shown in Figure 3.3(b) [86]. In the present studies, $\mathrm{CF}_{4}$ based plasma chemistry was selected.

The $\mathrm{CF}_{4}$ based plasma produces variety of neutral species $\left(\mathrm{F}, \mathrm{CF}\right.$ or $\left.\mathrm{CF}_{\mathrm{x}}\right)$ as well as ionic species $\left(\mathrm{F}^{+}\right.$, $\left(\mathrm{CF}_{x}^{+}\right)$, which interact with the surface. The ionic species etch physically by energy and momentum transfer. 
The overall effect of the plasma etch was to selectively remove Si from the surface or near surface layers of the SiC [46].

In our studies, the etch chamber was cleaned using oxygen plasma for 10 minutes with 600W ICP, $80 \mathrm{~W}$ RIE and $98 \mathrm{sccm}$ of Oxygen. The process was used to remove contamination from the previous etch process and the chamber was preconditioned for 12 minutes using our standard etch recipe with a 4" Si carrier wafer without any sample. Finally, SiC samples were mounted on a 4" clean carrier Si wafer using cool grease (conductive paste) and the etch process was performed.
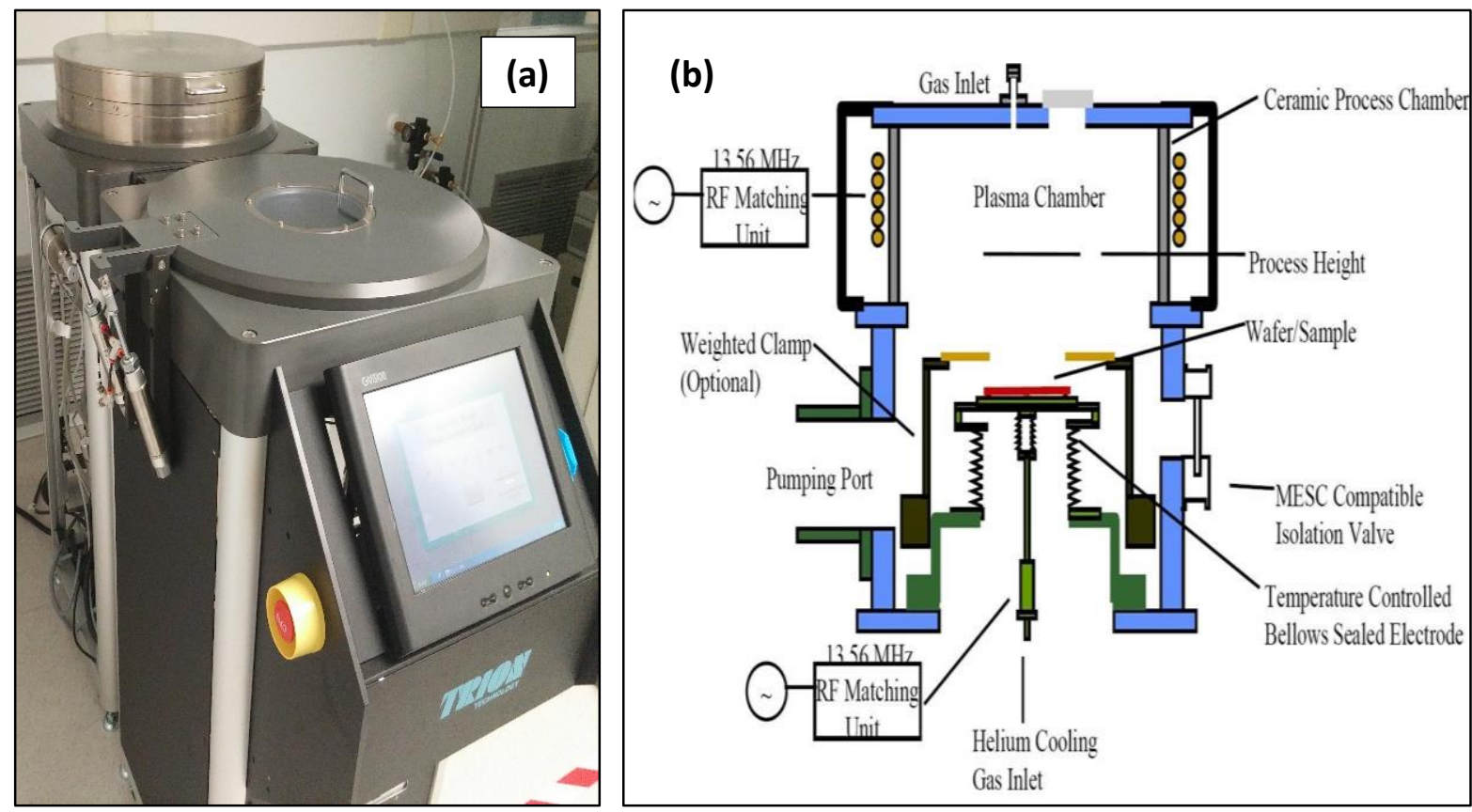

Figure 3.3: (a) Minilock-Phantom III ICP-RIE system and (b) Schematic of ICP-RIE [86].

The SiC etch parameters used in these studies were $600 \mathrm{~W}$ ICP power, 25 mTorr pressure and 20 sccm of $\mathrm{CF}_{4}$. The RIE power was varied based on the number of graphene layers desired of and a process time of 12 minutes was used for most of the samples (as explained later in section 4.1). After etching, the back of the SiC sample was cleaned with acetone to avoid contamination from the paste.

\subsubsection{Annealing Systems}

Two types of annealing system were used in these studies. They were an ultrahigh vacuum system (UHV) and rapid thermal annealing (RTA). The UHV system consists of a growth chamber which annealed $\mathrm{SiC}$ (0001) samples at temperatures of $950^{\circ} \mathrm{C}$ and collected RHEED data. This chamber was always kept at base pressure of $2 \times 10^{-9}$ Torr. Two turbo molecular pumps, a Varian model -V 550 and Leybold model 
360 were used to pump the growth chamber. The RHEED system was incorporated in to the growth chamber to monitor surface structure at various stages. It used KSA 400 version software. A Vecco atomic

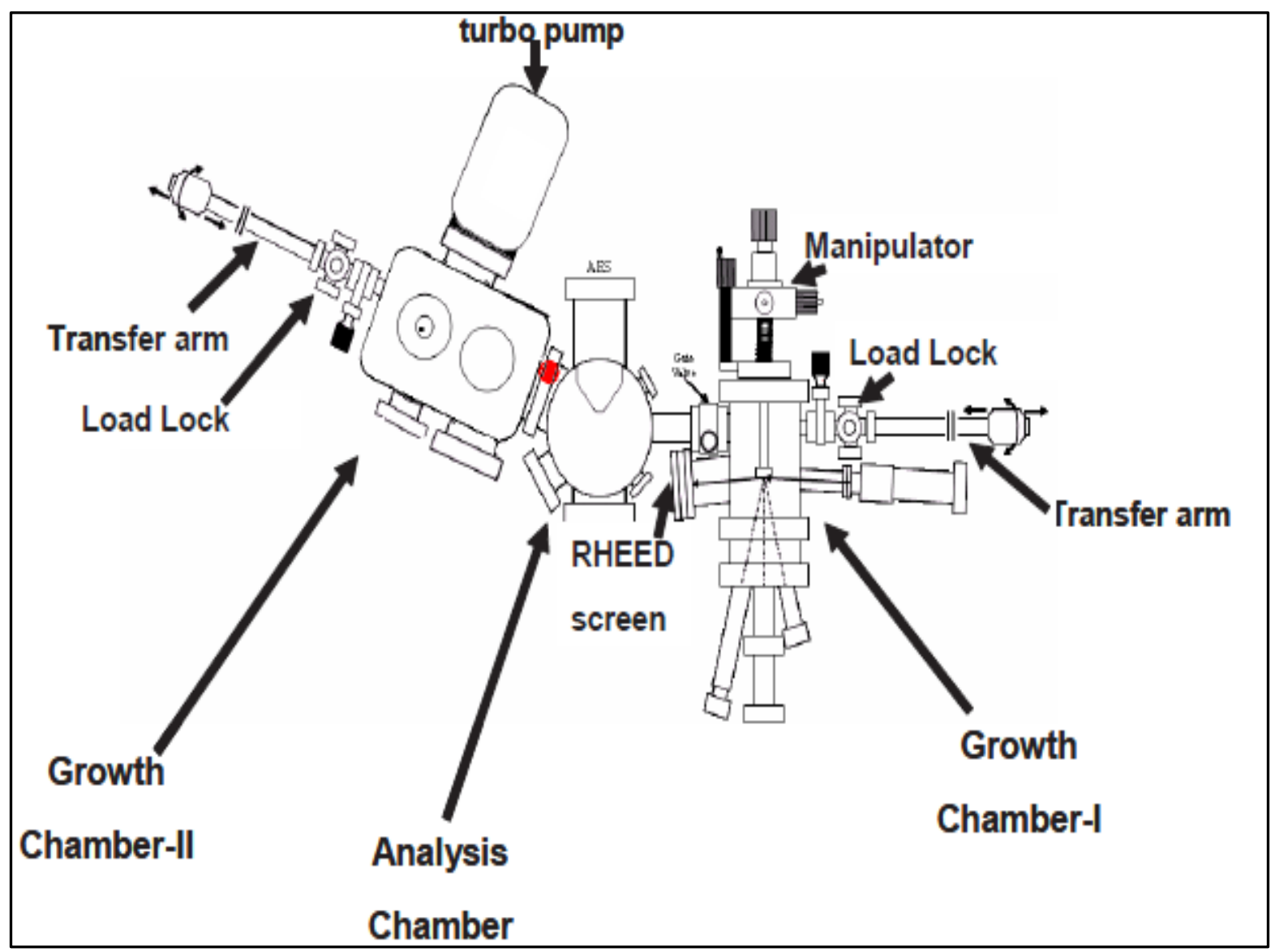

Figure 3.4: Schematic diagram of the UHV system, built in surface and material studies lab.

hydrogen source, a Varian model 951-5106 leak valve and an applied EPI model EPI-10-HT high temperature effusion cell were located on the source flange. In addition to these devices, a fissons sample manipulator with a built in sample heater was also present inside the growth chamber. Peng has given a complete description of the sample mounting, heater, heater power supply, thermocouple and infrared optical pyrometer used to control the sample temperature [83].

The sample was loaded in the chamber and after pumping down to $\sim 10^{-9}$ torr, it was heated to $300^{\circ} \mathrm{C}-400^{\circ} \mathrm{C}$ and allowed to degas and desorb any water vapor from the surface. The sample was then ramped to $944^{\circ} \mathrm{C}$, with a rate of $180^{\circ} \mathrm{C} / \mathrm{min}$ and maintained at annealing temperature for 1 hour before being cooled. The annealing profile for the UHV system is shown in Figure 3.5. This represents a typical UHV annealing sequence with the maximum heating rate of $\sim 50^{\circ} \mathrm{C} / \mathrm{min}$. 


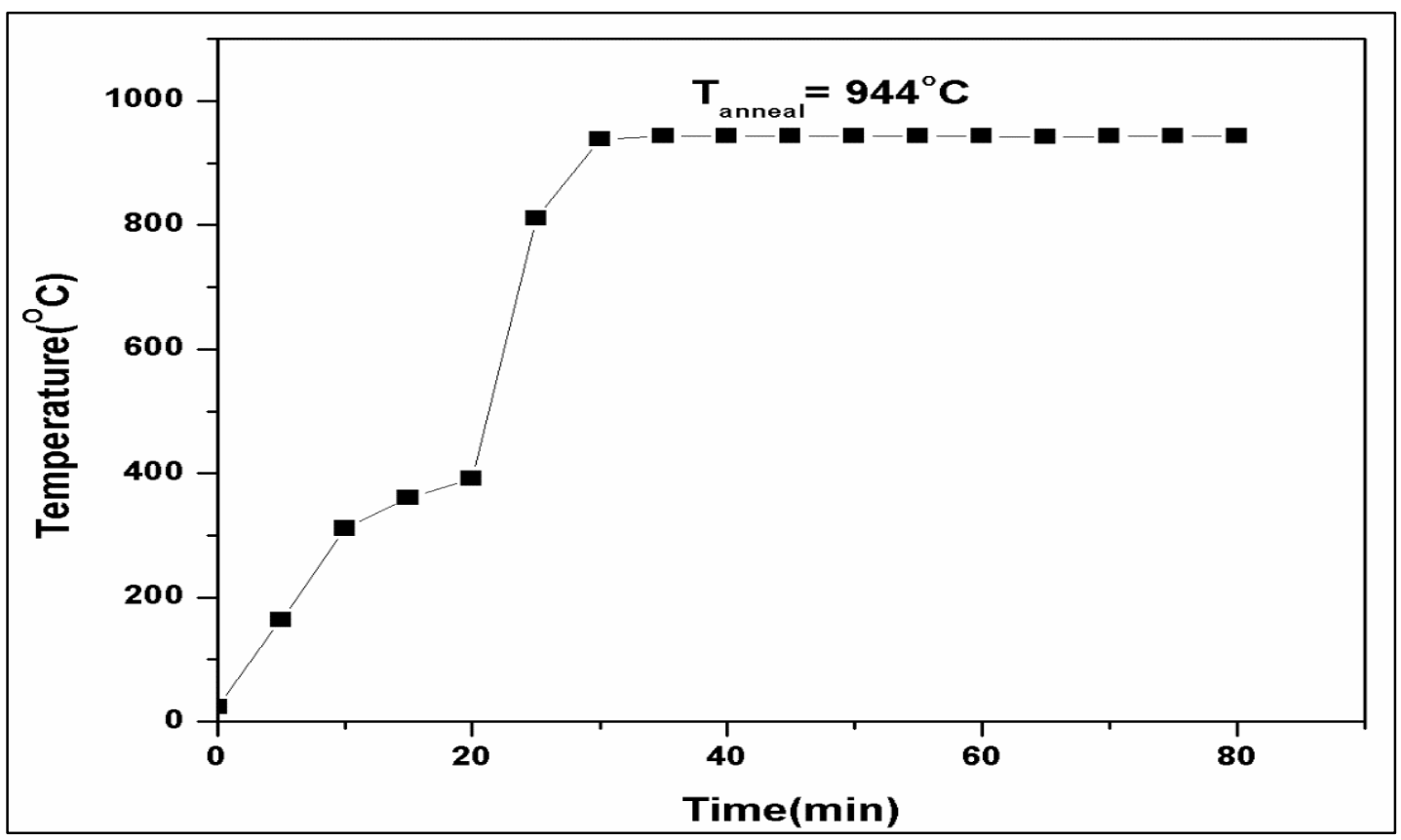

Figure 3.5: UHV annealing profile with respect to time.

The rate limiting step in the UHV annealing process was the pump down and this took about 18 hours. This was one of the motivating factors that led to the development of the rapid thermal annealing (RTA) process.

The RTA apparatus, as shown in Figure 3.6, has of a quartz flow tube which could be purged with ultrahigh purity inert gas. A thermocouple housed in the thermocouple shield was used to monitor the

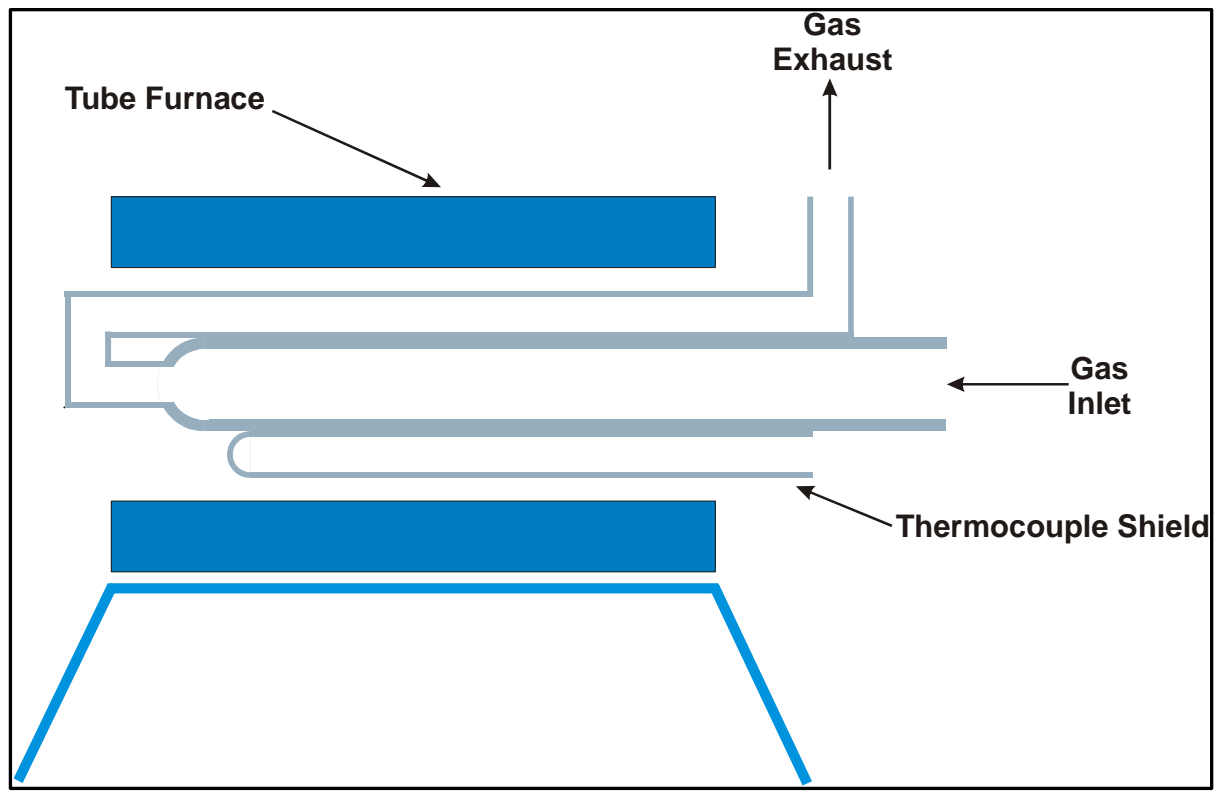

Figure 3.6: Schematic diagram of a RTA system showing the quartz tube with gas inlet, outlet and thermocouple shield. 
temperature of the sample. A second thermocouple, located near the furnace heating element was used to measure furnace temperature. For the annealing, the furnace was brought to a temperature slightly above the desired annealing temperature $\left(950^{\circ} \mathrm{C}\right)$. After loading the samples into the tube, the system was purged with Ar for an hour in order to remove oxygen contamination. The quartz tube with samples was then inserted into the furnace and rapid temperature increase in the sample was measured using the thermocouple. The typical plot of the temperature vs time is shown in Figure 3.7. The temperature

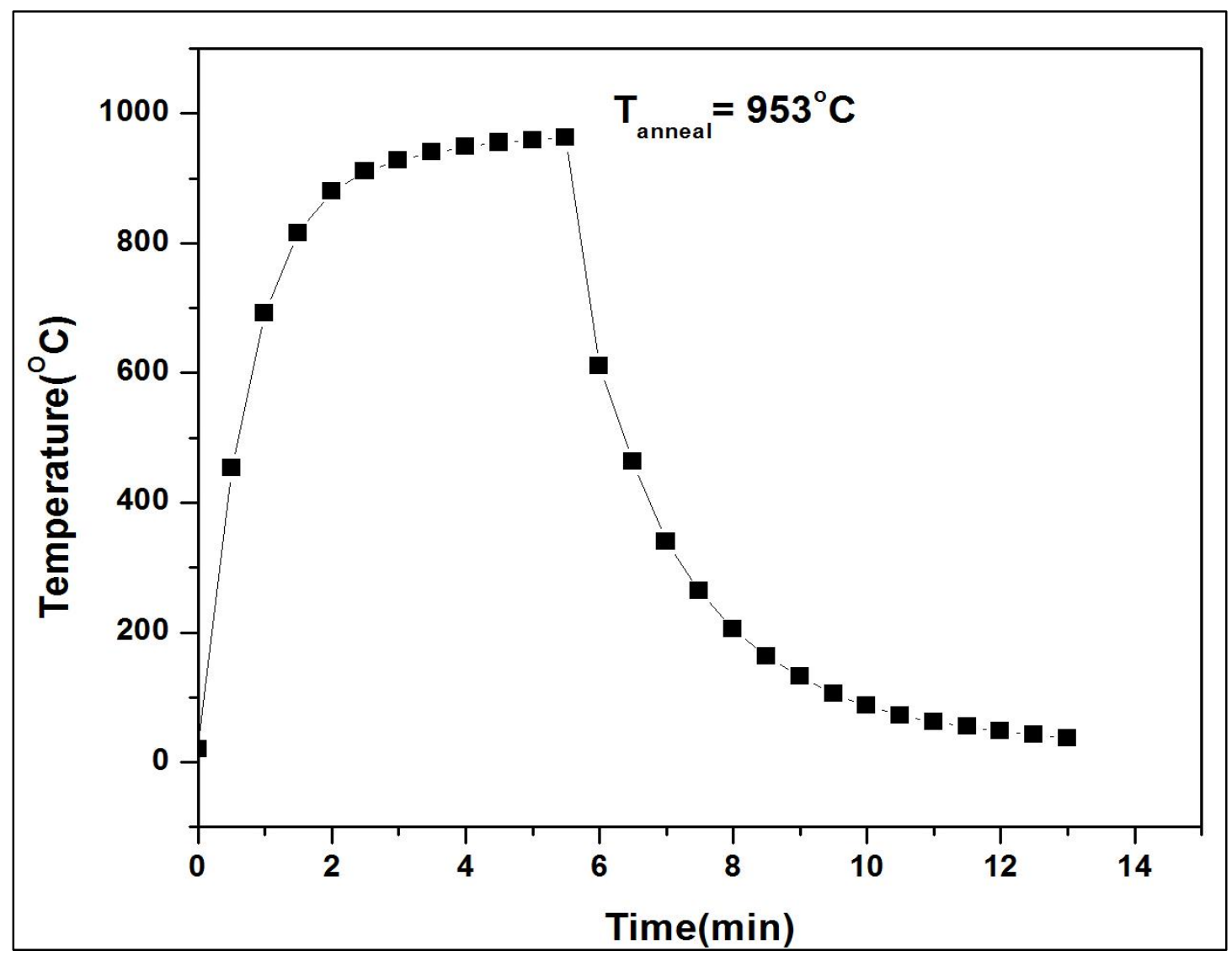

Figure 3.7: RTA profile with respect to time. The annealing time was measured from the time the temperature reached at $940^{\circ} \mathrm{C}$ and an average temperature of $953^{\circ} \mathrm{C}$ was obtained for 2 minute anneal.

increased to annealing temperature at a nominal rate of $450^{\circ} \mathrm{C} / \mathrm{min}$. Over the last two minutes of the ramp, an average temperature of $953^{\circ} \mathrm{C}$ was achieved. The tube was then rapidly withdrawn quickly to cool samples.

The above annealing system gave several advantages over the conventional UHV system. Since the process was carried out at atmospheric pressure, no pumps are required for the annealing. Secondly, high ramp rates were easily achieved as compared to the slower ramp rates of UHV annealing. In addition, 
multiple samples could be annealed in the RTA system. All these improvements made the graphene synthesis faster and easier to control.

\subsubsection{Electron Beam Evaporation}

Physical vapor deposition (PVD) is a thin-film deposition process in which a material (metal, alloy, compound, or composite) is either evaporated or sputtered onto a substrate surface in vacuum. The chemical composition of the deposited material is not altered in the process. PVD can be used to deposit most metals and some dielectric materials. Dielectric films after PVD deposition generally have poor insulating capability because of holes and defects in the films. There are three typical methods for PVD: e-beam evaporation, thermal evaporation, and sputtering deposition. An E-beam evaporator is used normally to coat samples with various metals. A thermal evaporator is chosen when materials (like photoresists and Ebeam resists) on the substrate are sensitive to $x$-ray radiation from the E-beam. These two methods deposit materials only on the surface that is facing the evaporated material source and are good for metal liftoff deposition. Sputtering deposition has a very good step coverage over uneven structures and is good for trench filling.

In the cleanroom of WVU, an e-beam evaporator was used to deposit Pd/Au, $\mathrm{Al}$, and Ti/Au, $\mathrm{Cr} / \mathrm{Au}$, or Pd-Ni contact pads. The metallization for these studies was done by e-beam evaporation. This method was preferred over sputtering because it allowed contacts to be deposited on sample surfaces with a minimum amount of damage to the thin film. In e-beam evaporation, a beam of electrons is generated and focused on the target of the desired metal source. The electron beam beats the target surface and produces a flux of evaporated material. The gaseous material strikes on the surface and forms a thin solid film in the openings.

Two different e-beam evaporators were used in these studies: a Temescal BJD-200 and a custom built Kurt Lesker unit. These are located in the SRF cleanroom and are as shown in the Figure 3.8 and 3.9. In the Temescal evaporator, the sample was loaded on the sample holder and the system was pumped down to its operating pressure of 1 microtorr using mechanical pumps and cryopumps. Control software was used to select the metal and thickness to be deposited. 10 micron thick Ti and 100 micron thick Au were deposited for all these processes as it was found to be optimum for wire bonding. After the operating pressure was reached, the emission current was increased from zero till the desired thickness was achieved. Beam voltage was kept constant at $10 \mathrm{kV}$ for all metallization processes. The control program automatically controls the opening and shutting of the chamber, once the desired thickness is achieved. The chamber is then cooled down to $40^{\circ} \mathrm{C}$ and the samples were removed after bringing the chamber to 
atmospheric pressure in the Kurt Lesker evaporator, everything was controlled by a programming recipe for the given metal and thickness. The contacts with the photoresist were then exposed to acetone for liftoff.

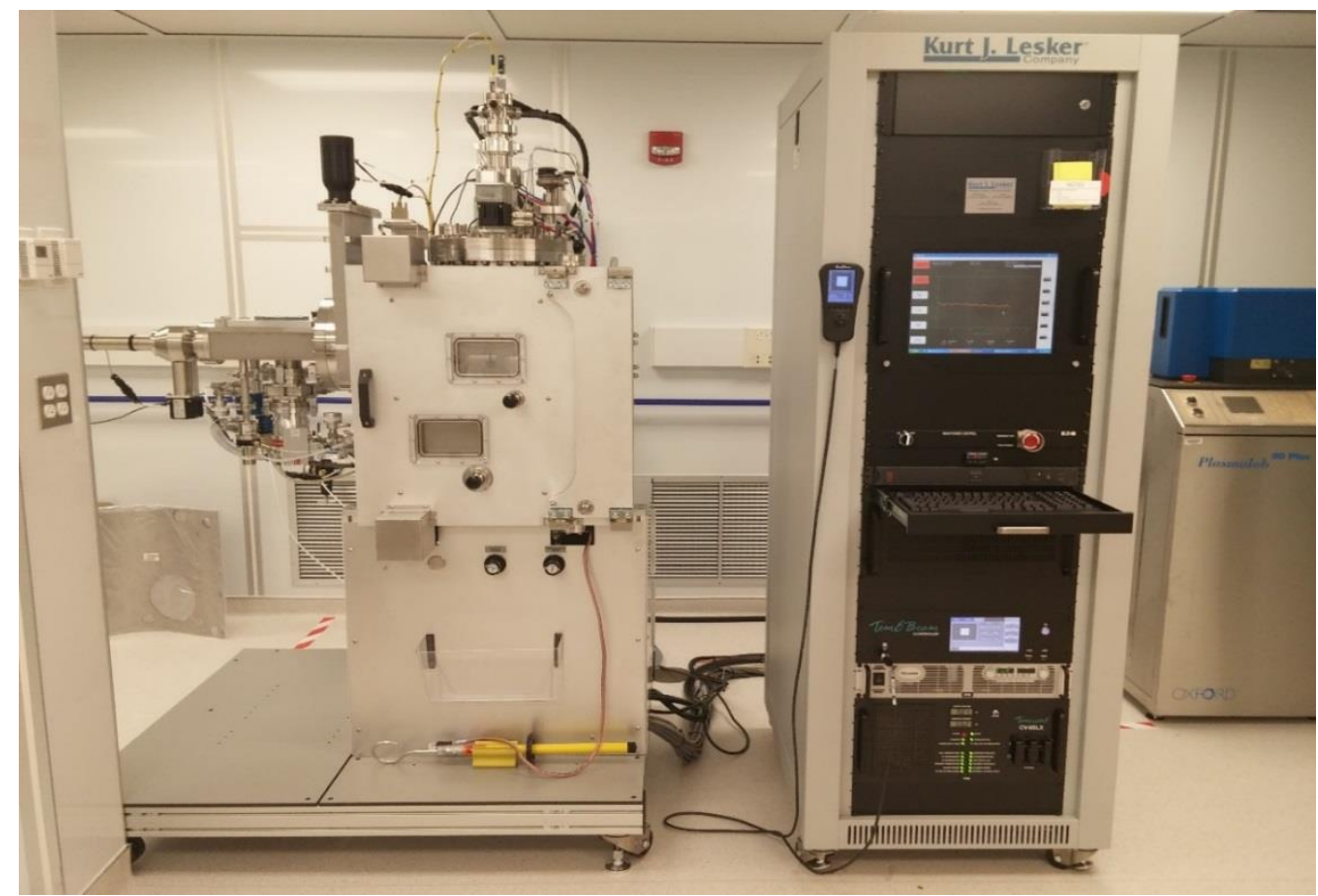

Figure 3.8: Kurt Lesker E-beam evaporator used to deposit electrical contact in the Shared Research Facilities cleanroom.

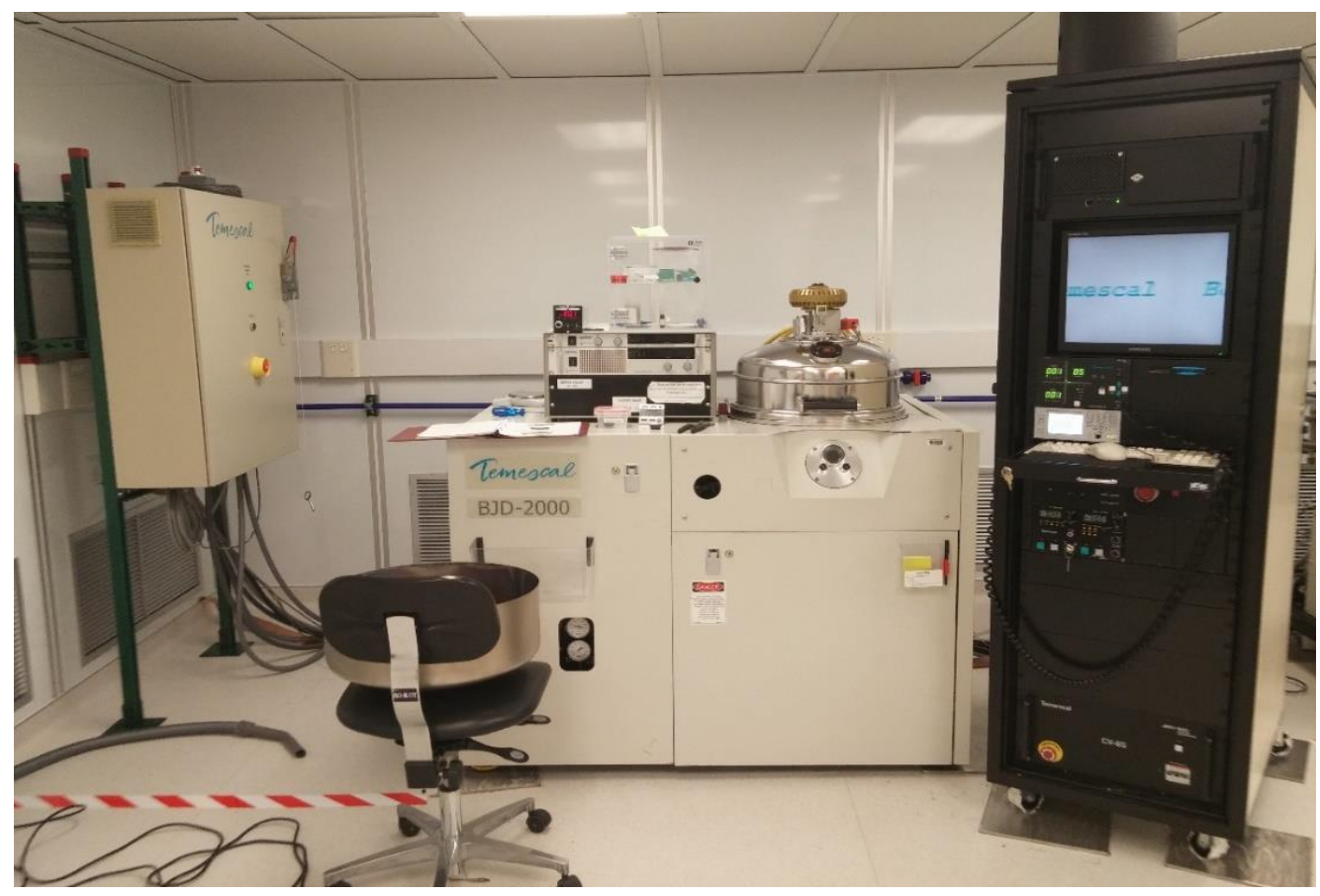

Figure 3.9: Temescal BJD200 E-beam evaporator used to deposit electrical contact in the shared Research Facilities cleanroom. 


\subsubsection{Wafer Dicer}

A wafer dicer is typically used to dice semiconductor wafers into individual die. For the use of wafer into packaging, precision cutting of the wafer is needed with minimal contamination. Conventional saws require adhesives and thus chances of contamination are high. The wafer dicer uses a vacuum chuck to hold the sample on a tape and the particulate produced during the dicing can be washed away using a controlled flow of water inside a close chamber.

The wafer dicer used in our studies was the DAD3240, DISCO, Inc. model as shown in Figure 3.10 [87] . Diamond blade (ZH05_SD2000 manufactured by DISCO) were used for dicing SiC. Vacuum holds either $5 \mathrm{~cm}$ circular wafers or $1 \mathrm{~cm}$ rectangular sensors. The dicing wheel had a rotation speed of 30,000 $\mathrm{rpm}$ and a feed rate of $10 \mathrm{~mm} / \mathrm{s}$. The samples were aligned using a built-in microscope for precision cutting between the sensor patterns in both $\mathrm{x}$ and $\mathrm{y}$ directions. Deionized water is flowed while dicing to keep the sample free from particles and chips.

In our studies, the wafer dicer was used first to dice the $5 \mathrm{~cm} \mathrm{SiC}$ wafer into $1 \mathrm{~cm} \times 1 \mathrm{~cm}$ squares for graphene synthesis and device array fabrication and then to dice the $1 \mathrm{~cm}$ wafer into individual sensors for surface modification and gas testing.

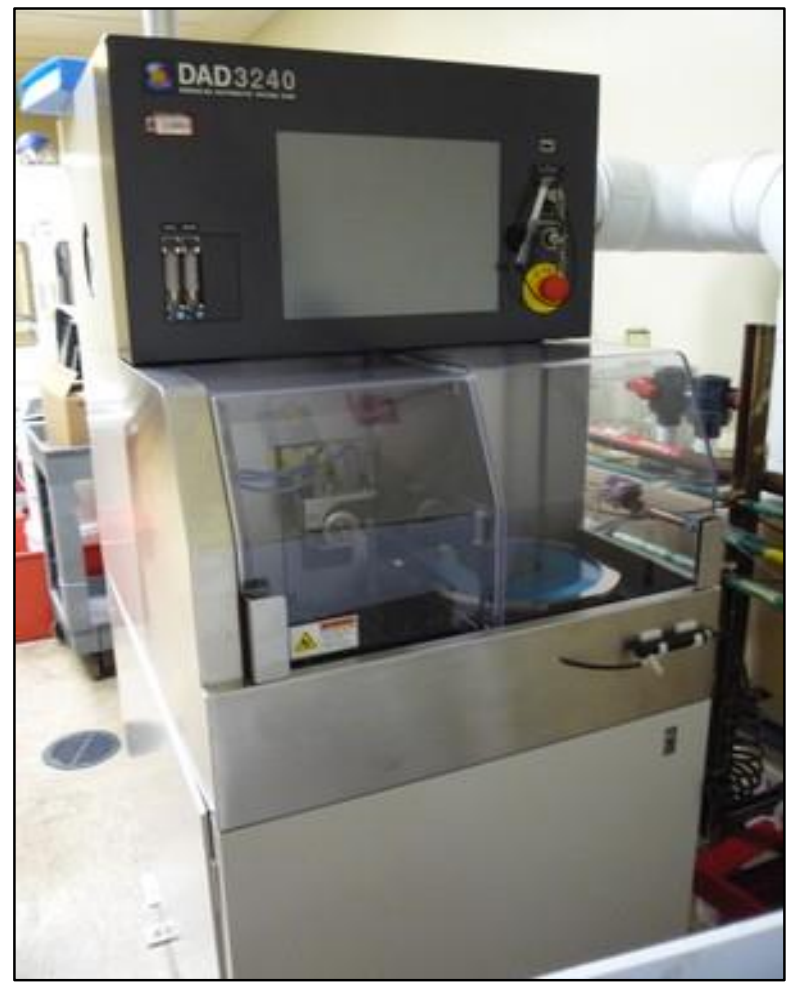

Figure 3.10: DISCO wafer dicer, capable of dicing substrates with great accuracy and minimum contamination. 


\subsubsection{Wire Bonder}

The wire bonder is designed to attach fine wire $(100 \mu \mathrm{m})$ leads between the contact pads of individual devices and larger electrical leads and contacts. As the testing of graphene sensors involves measurement of I-V characteristics, it is required to make good electrical contact between the sensor and package. These bonds should also be strong enough to withstand environmental conditions like high gas flow rates, high temperatures and the mechanical stress during the gas sensor setup.

The wire bonder in our studies was West Bond 74776E, located in SRF cleanroom, as shown in Figure 3.11. Gold wires were used for all experiments as they are more stable and can resist oxidation as well as harsh environments like high temperature and corrosive gases. All the bonds used were ball-wedge bonds i.e. forming a ball of gold at the first point of contact, followed by wedge on the other. Ultrasonic power created the ball at the first point of contact and up to six inches of wire length can be used for bonding. The substrate heating and time were adjusted in order to make strong bonding between the wire and the contacts, which can be controlled based on the applications and substrates. Typical conditions for these wire bonds are $200 \mathrm{~W}$ ultrasonic power and 20 ms ultrasonic time.

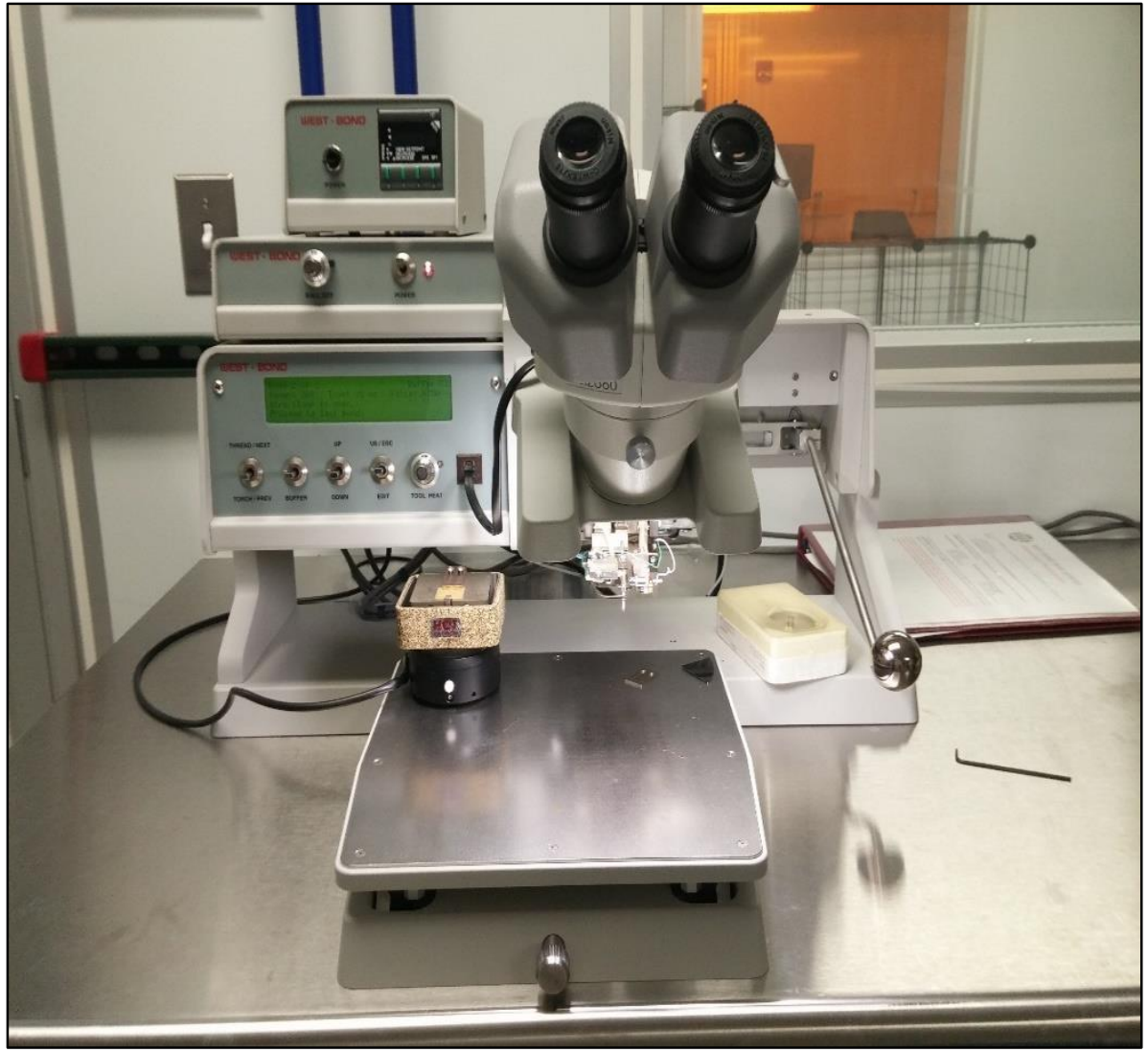

Figure 3.11: Wire bonder used for gold wire bonding between the sensor platform and the packaging. 


\subsection{Characterization}

\subsubsection{X-ray Photoelectron Spectroscopy (XPS)}

X-ray photoelectron spectroscopy (XPS) is a surface sensitive spectroscopic technique. In this technique, material surface is irradiated with $\mathrm{Al} \mathrm{K}_{\alpha}(1483.6 \mathrm{eV})$ or $\mathrm{Mg} \mathrm{K}_{\alpha}(1250 \mathrm{eV})$ radiation to eject the core level electron from the atoms of the sample. The kinetic energy of the photoelectron is determined by the energy of $\mathrm{X}$-ray radiation, hv, the electron binding energy, $\mathrm{E}_{\mathrm{b}}$, and the work function of the spectrometer by the relation shown in Figure 3.12 [88].

As the electron binding energies are dependent on the chemical environment of the atom, XPS investigates the bonding of the atoms in the sample. A simple Beers law can be used for elemental analysis which proves that as sampling depth of photoelectron is on the order of 3 times the electron mean free path. Figure 3.13 show a plot of the escape depth as a function of electron kinetic energy [89].

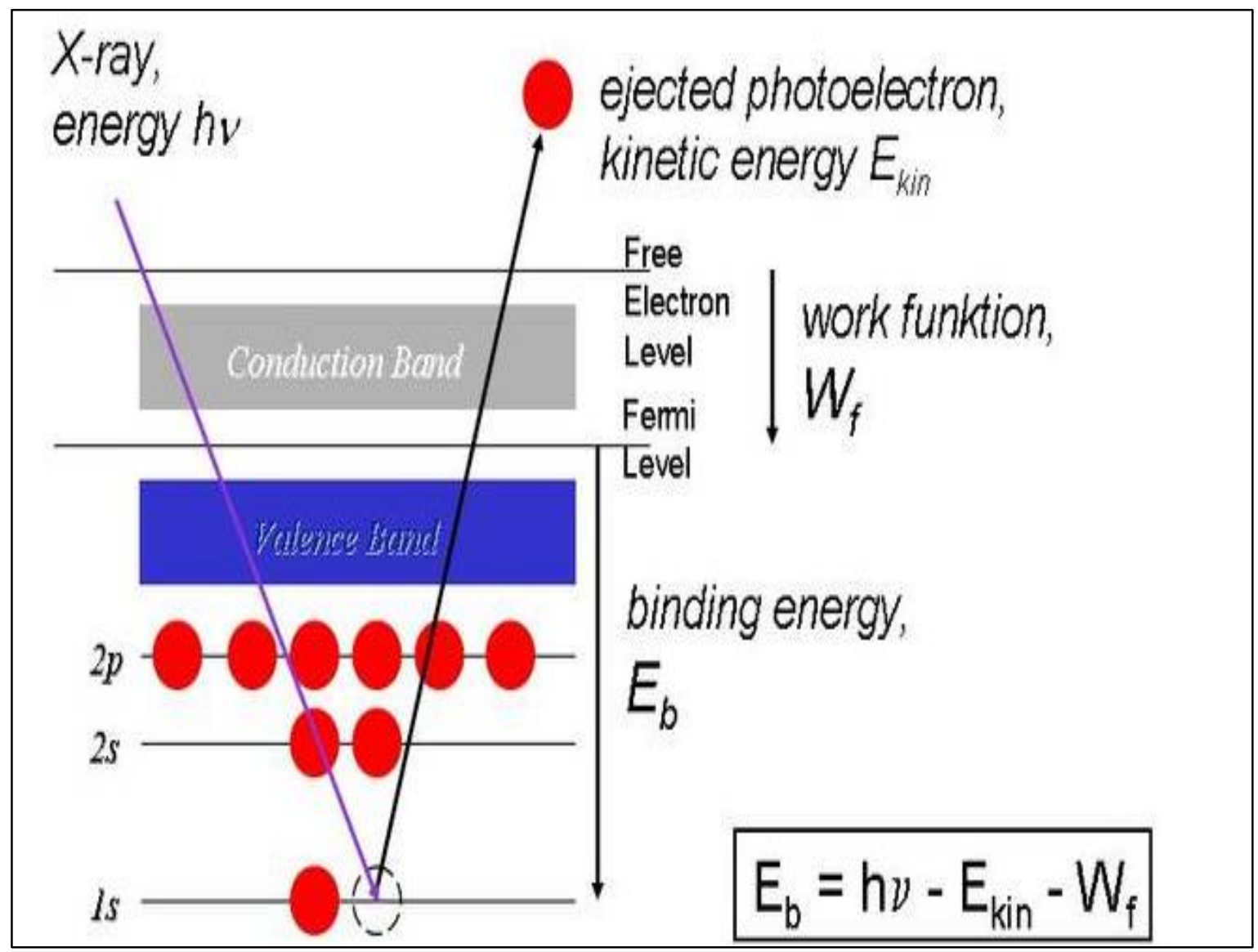

Figure 3.12: The XPS process showing ejection of photoelectron due to incident X-ray, resulting in emission of electron with characteristic kinetic energy [88]. 
The mean free path can be related to the electron energy for inorganic compounds by the expression given by Seah and Dench, as shown below [90].

$$
\lambda=2170 E^{-2}+0.72(a E)^{1 / 2}
$$

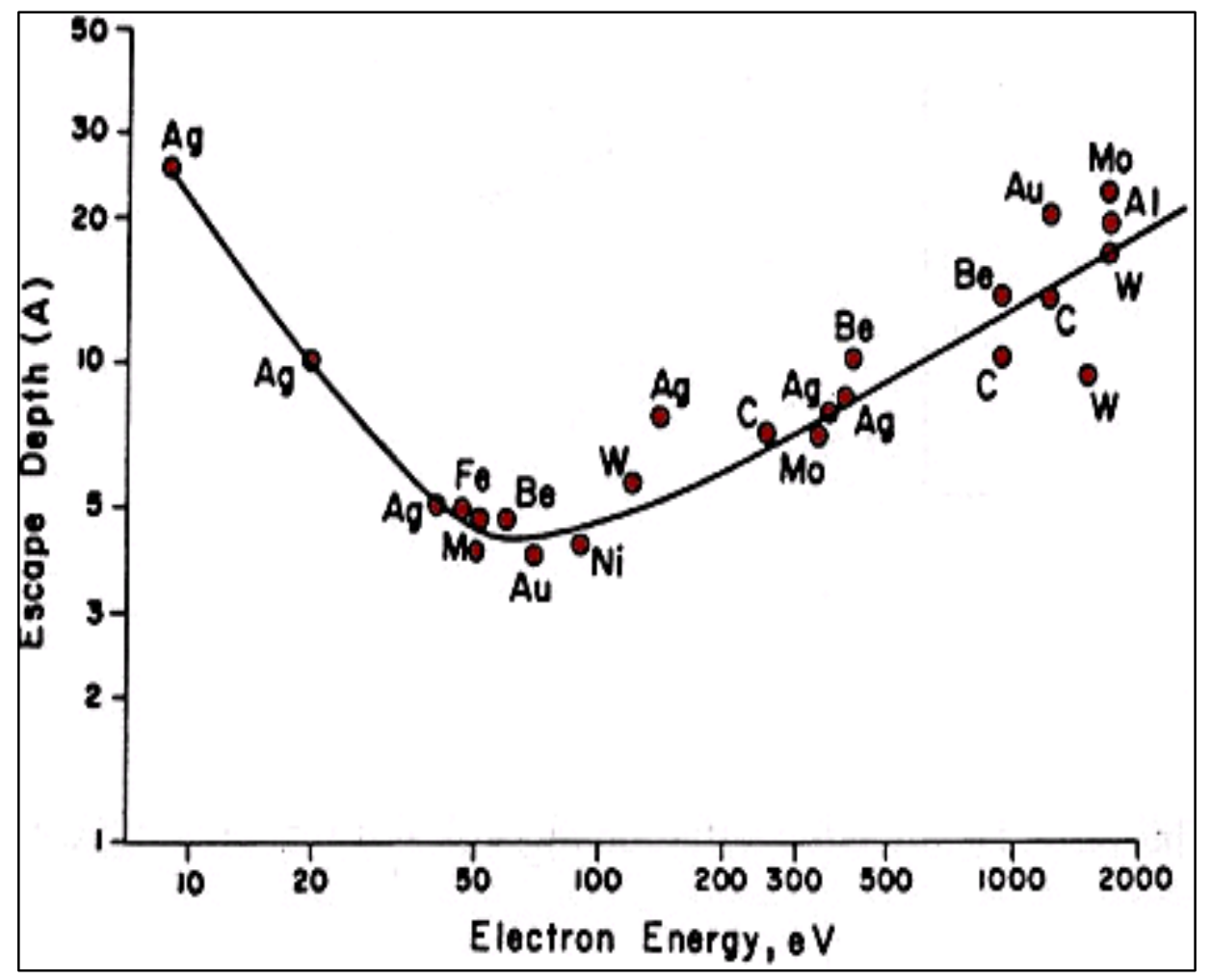

Figure 3.13: Escape depth (mean free path) of electron versus electron kinetic energy [89].

The XPS analyses for this dissertation employed a PHI 5000, located in the B62 in Engineering Sciences Building. A monochromatic Al source was used for XPS data and $15 \mathrm{kV}$ X-ray voltage was applied for production of $x$-rays. The emitted photoelectrons were analyzed be means of a hemispherical electron energy analyzer. For survey spectra, pass energy of $117.4 \mathrm{eV}$ was used, while high resolution spectra pass energy of $23.5 \mathrm{eV}$ was used. The instrument was calibrated using the Au4f $\mathrm{f}_{7 / 2}$ spectral line at $84 \mathrm{eV}$ [91].

An ion pump for the main chamber maintained a base pressure of $10^{-8}$ Torr, while a turbo pump was used to maintain the vacuum in the sample introduction chamber. The sample was mounted on a molybdenum puck and loaded into the introduction chamber. Once the pressure in introduction chamber was reduced to $1.6 \times 10^{-6} \mathrm{Torr}$, the gate valve between the introduction and main chamber was opened 
and the sample was transferred by means of a transfer arm. The acquired spectra were analyzed and processed using PHI multipack software.

\subsubsection{Raman Spectroscopy}

Raman spectroscopy is a technique can observe vibrational, rotational and other low frequency modes in a system [92]. It is based on the principle of interaction of monochromatic light with a given sample. The photons of the focused light beam which lose energy are shifted to longer wavelength (strokes shift), while those that gain energy are shifted to shorter wavelength (anti-strokes shift). For our study, a Renishaw micro-Raman spectrometer was employed, as shown in Figure 3.14.

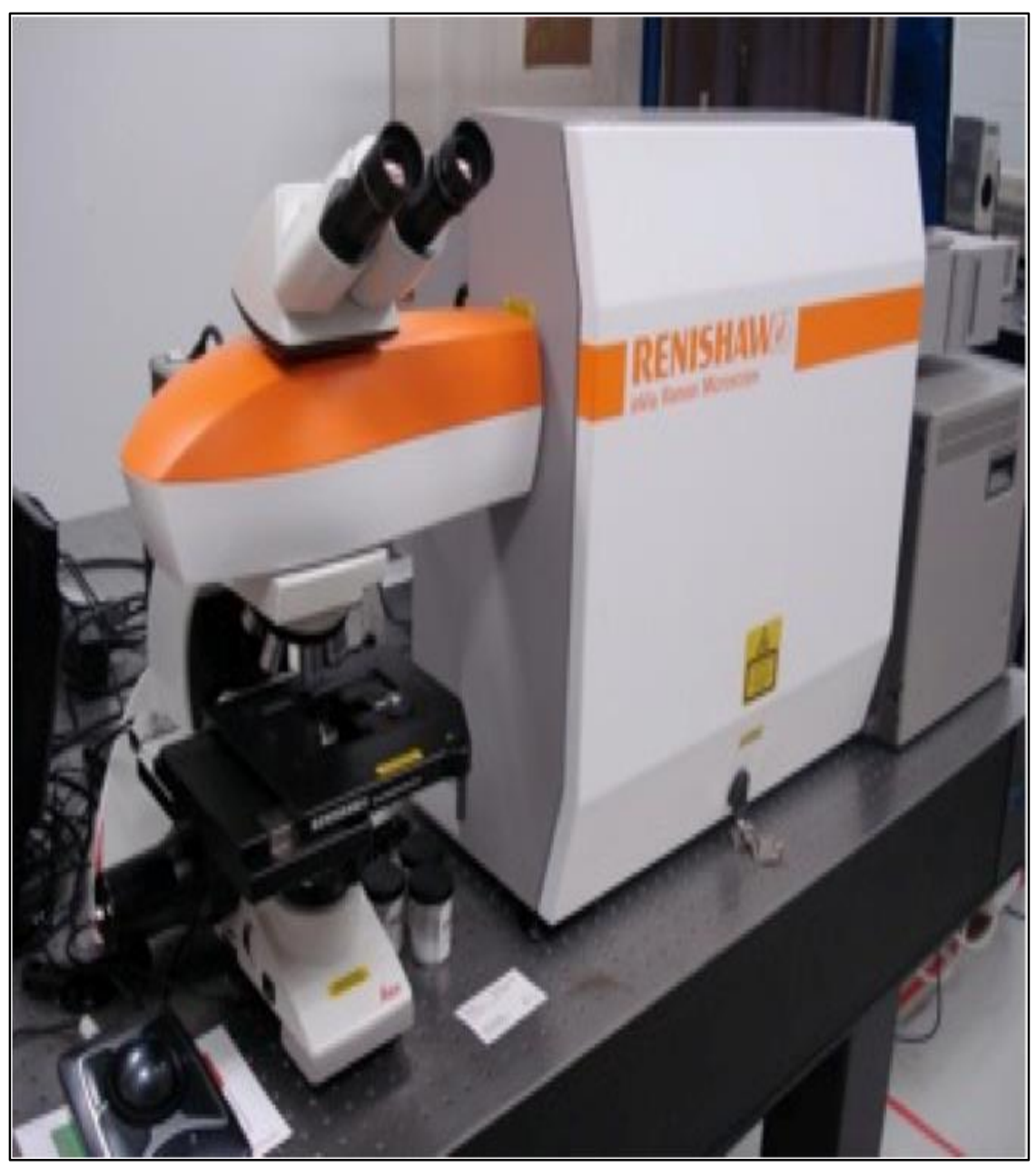

Figure 3.14: Renishaw micro-Raman spectrometer used, with the $532 \mathrm{~nm}$ green laser and $1 \mu \mathrm{m}$ spot size.

In all the studies, a $532 \mathrm{~nm}$ green laser and a spot size of 1 micron was selected. The power was kept at $100 \%$ in order to avoid damage to the graphene thin films. Typical Raman spectra of HOPG and monolayer graphene flake, derived from HOPG, are shown in Figure 3.15 [93]. 


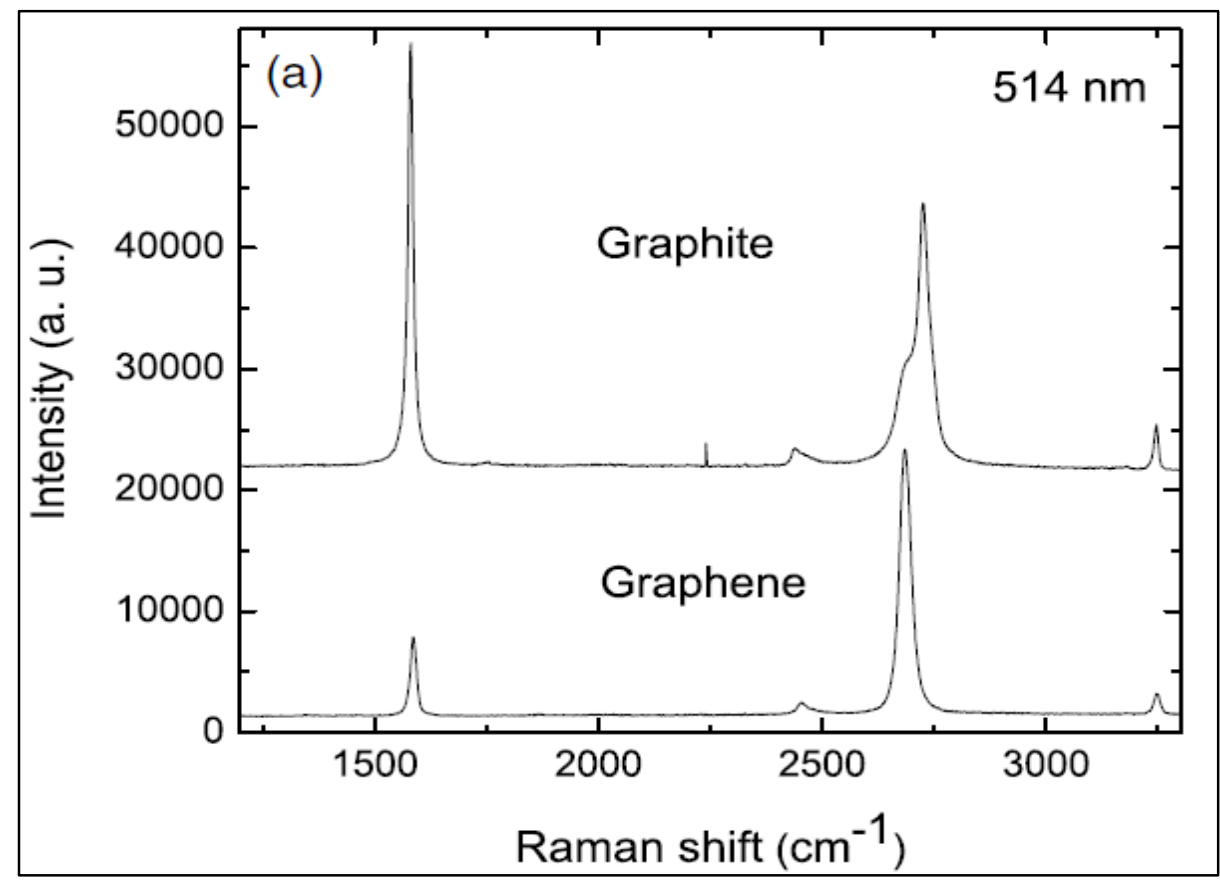

Figure 3.15: Raman spectra comparing of the bulk graphite and monolayer graphene.

\subsubsection{Reflection High Energy Electron Diffraction (RHEED)}

Reflection high energy electron diffraction (RHEED) is widely used to monitor the structure and atomic spacing after growth or etching of thin films. The high energy beam is directed at the sample at a glazing angle. Electrons from the surface are diffracted by the crystal structure of the sample and then collected on a phosphor screen mounted opposite to the electron gun. The resulting pattern is a series of spots or streaks, as shown in Figure 3.16, which is the RHEED pattern for 6H-SiC (0001) surface after cleaning. The distance between the streaks is related to lattice spacing. The intensity of the pattern depends on the sample surface. Flat surface produces sharp RHEED patterns, whereas rougher surface produces diffused pattern.

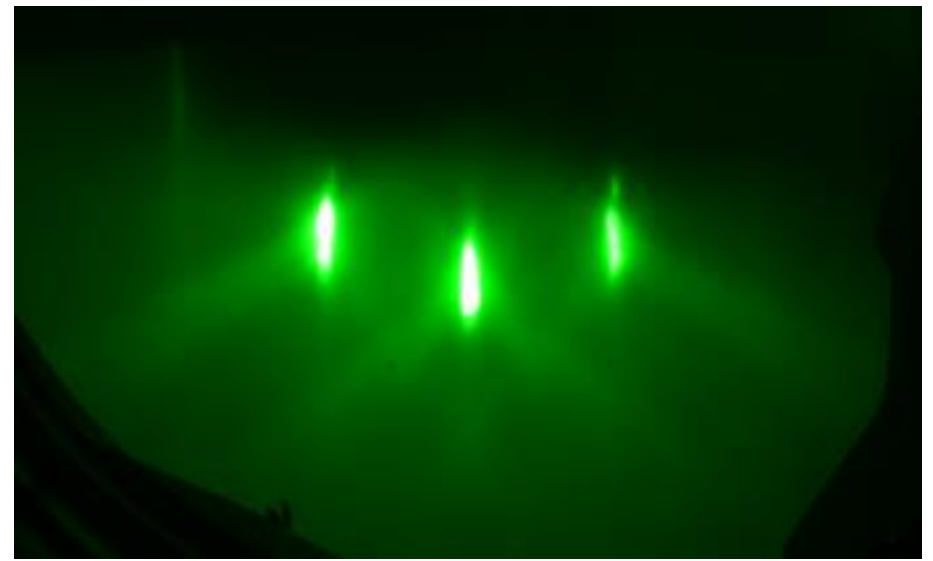

Figure 3.16: RHEED image of as received 6H-SiC (0001) surface after clean taken along [110] 


\subsubsection{Atomic Force Microscopy (AFM)}

Atomic force microscopy (AFM) consists of a laser, cantilever, photodetector, controller, scanner and computer. A red laser and a photo detector can track the movements of the cantilever. The scanner is used to move the sample or the tip. The controller moves the scanner and maintains the feedback loop. Most common scanners are piezoelectric tube scanners. The force transducer is the deflecting cantilever on which a tip is mounted as shown in the Figure 3.17. As the cantilever and tip assembly are scanned over the surface, reflected laser light from the cantilever is collected using a photodetector and the morphology of the surface can be determined.

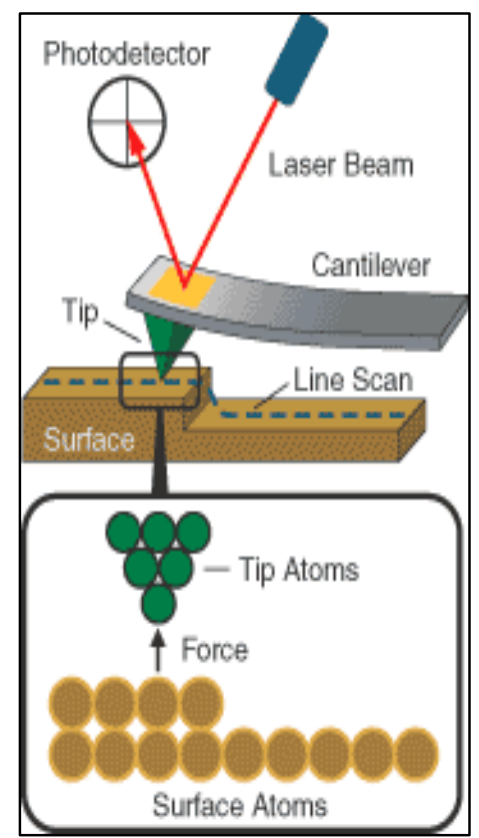

Figure 3.17: Schematic diagram of the AFM, showing assembly of laser beam, cantilever, photodetector and substrate surface.

Both conducting and non-conducting materials can be imaged using AFM. As the tip-surface interaction might cause surface deformation, the scanning is done at the lowest possible forces. Two methods are typically employed in order to obtain the surface morphology, namely the contact and the tapping mode. In the contact mode, the tip is close to the surface and thus more force is applied between the tip and the surface. This type of mode is generally used for frictional studies of the surface. In the tapping mode, the tip is allowed to oscillate at a certain known frequency and relative changes in oscillation due to Van der Wall's forces from the substrate surface are measured. As the sample force applied in the tapping mode is less than contact mode, the tapping mode was used for imaging of the surface. 
Figure 3.18 shows the Agilent 5500 SPM AFM used in these studies. The system was calibrated using standards provided by Micromash, prior to measurements. The standards for in this AFM were 20 $\mathrm{nm}$ in height. As the AFMs has angstrom level sensitivity and a lateral resolution of about $2 \mathrm{~nm}$, even forces of the order of $\mathrm{nN}$ can be applied and interactions with surface can be imaged with great accuracy.

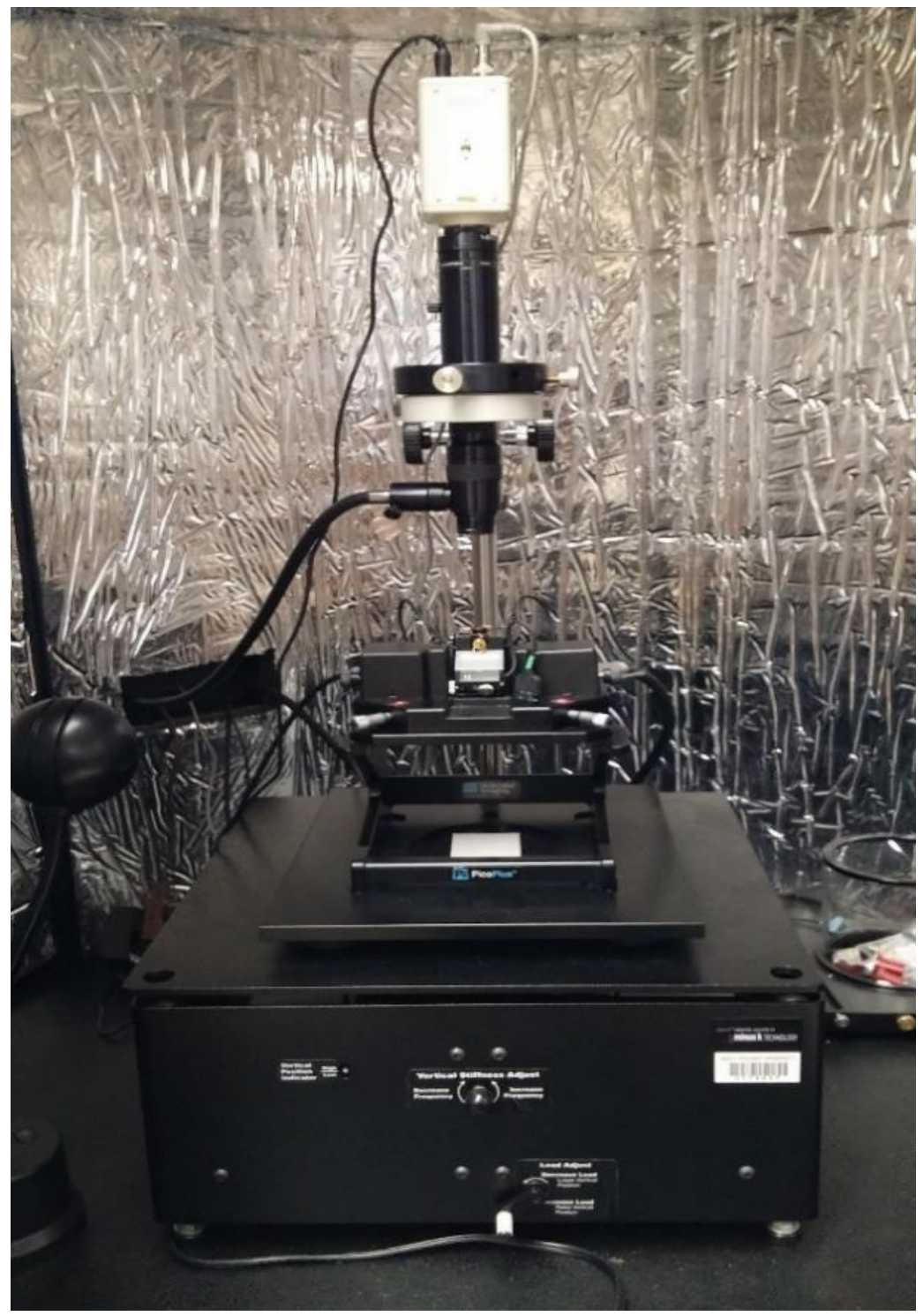

Figure 3.18: Agilent AFM used for understanding the morphology of the surface by imaging at ${ }^{\circ} \mathrm{A}$ resolution.

As the vibrations from the room can affect the interactive forces, a vibration isolation table inside a Herzan acoustic isolation hood was used to mount the AFM. $20 \%$ integral gain and $40 \%$ proportional gains were selected for most of the AFM studies to get noise-free images. The images were obtained via Pico View software, provided by Agilent and later analyzed by a scanning probe image processor (SPIP) and Gwyddion software. 


\subsubsection{Scanning Electron Microscopy (SEM)}

A scanning electron microscope (SEM) focusses a beam of electron for imaging the sample. The focused electrons interact with the sample and produce secondary electrons, which provide the topography information. Since the geometry and distribution of nanoparticles on the graphene surface needs to be studied for nanoparticle synthesis optimization, SEM imaged the topography prior to and after the nanoparticle nucleation. Samples were mounted onto copper tape and were imaged with a Hitachi S-4700 Scanning Electron Microscope (SEM) with a cold field emission electron source. Different voltage and resolutions were set based on the requirement of the samples.

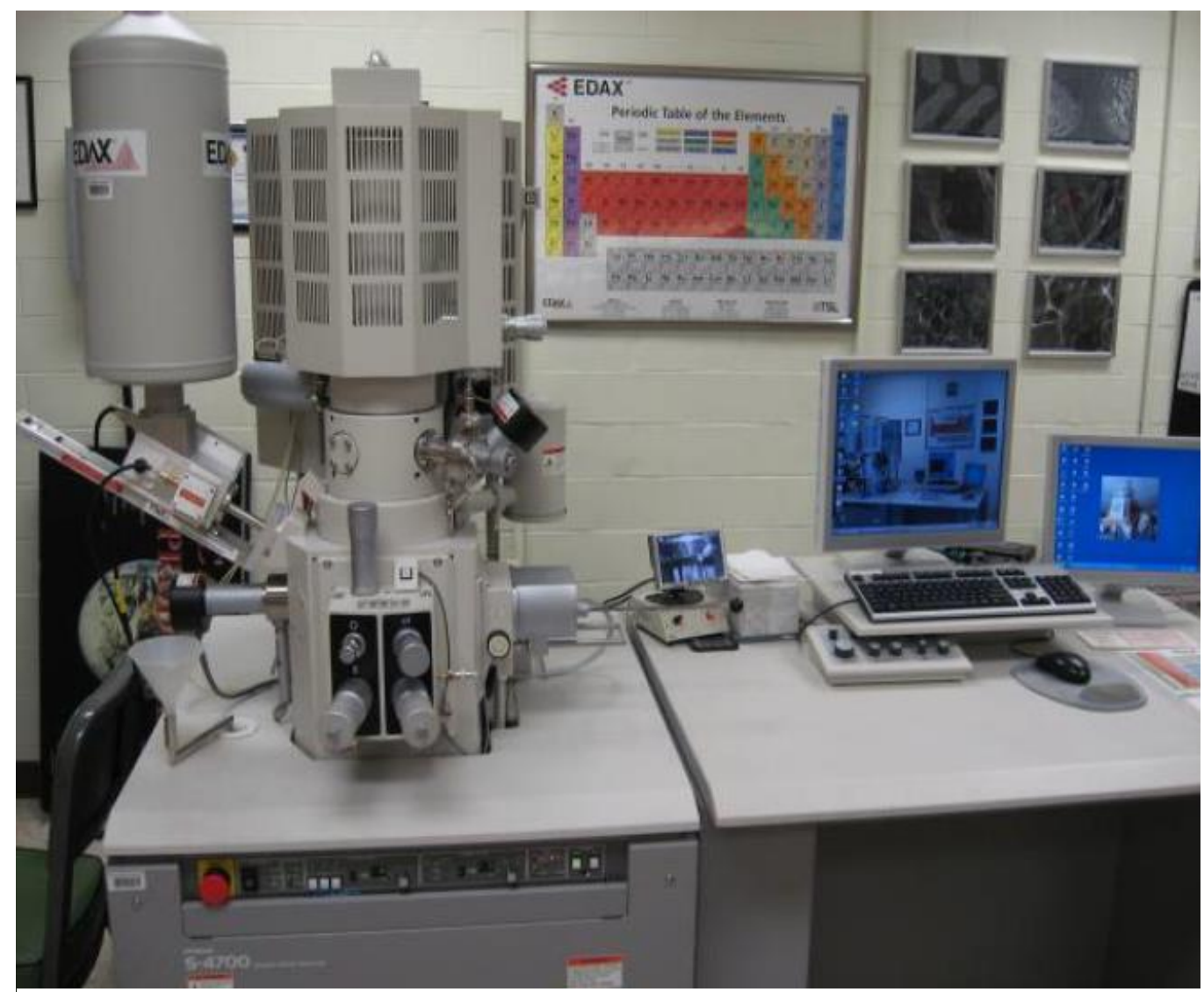

Figure 3.19: Hitachi S4700 Scanning electron microscope

\subsubsection{Electrical Characterization}

A known current is sourced and flowed through the unknown resistance. The voltage that develops across the resistance is measured and the resistance is determined by dividing the measured voltage by the sourced current, by means of Ohm's law is used: $\mathrm{R}=\mathrm{V} / \mathrm{I}$.

The measurements in the current work were taken on a probe station and either an Agilent 4156C precision semiconductor parameter analyzer or Keithley 4200 sourcemeter was used to obtain the data. 
A potential, which was varied from -20 to $20 \mathrm{~V}$ in steps of $0.1 \mathrm{~V}$, was applied by means of the semiconductor parameter analyzer and the current response was measured accordingly.

There are two ways to do electrical measurements- two point and four point [94].

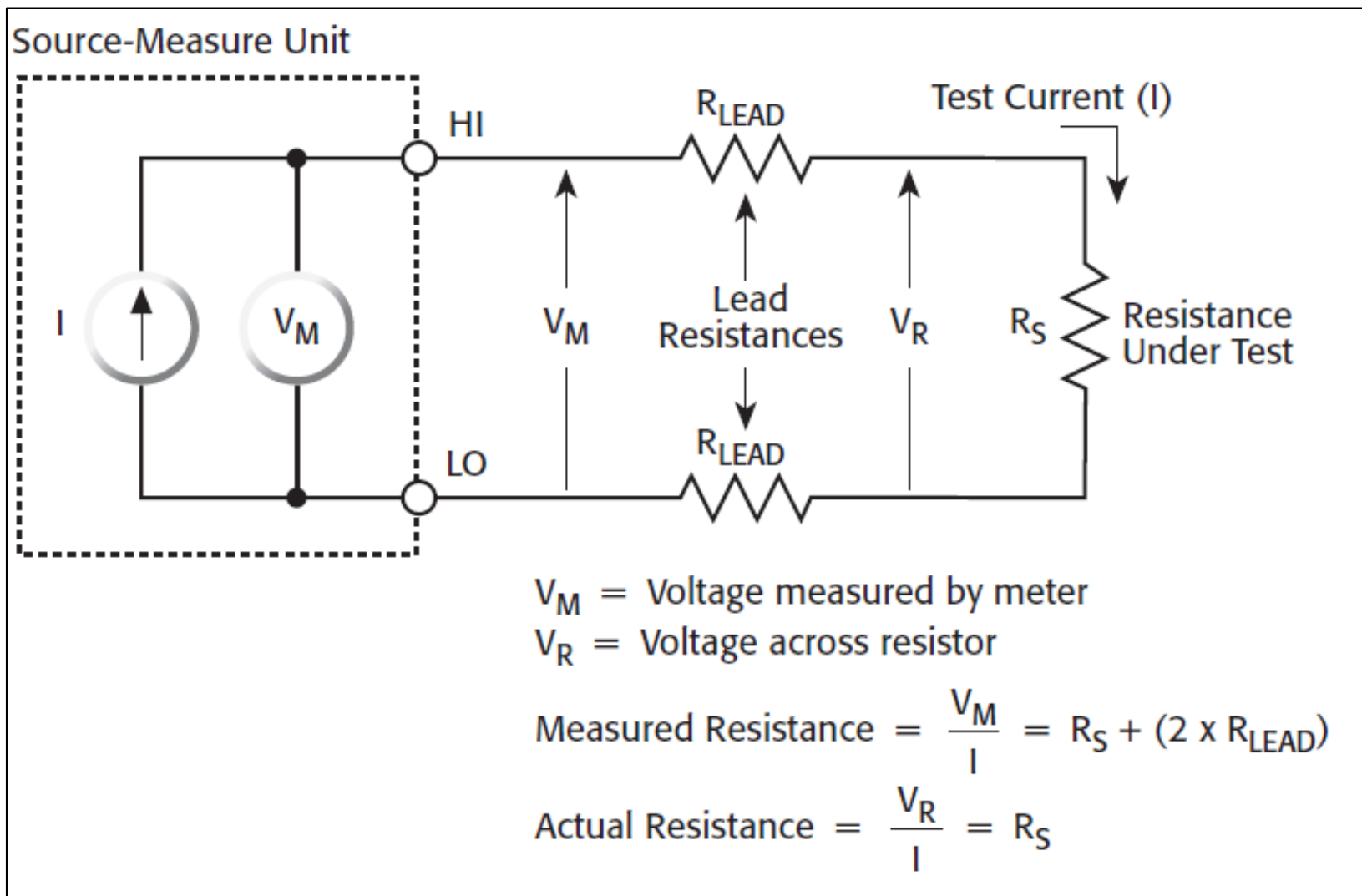

Figure 3.20: Two point resistance measurement configuration

As shown in Figure 3.20, the resistance measured in the two point method is the combination of the film resistance and the resistance of both the semiconductor/contact interfaces. These resistances can be obtained by measuring the total resistance as a function of contact separation.

In our studies, I-V measurements were used to determine basic contact characteristics (i.e. Schottky or Ohmic, carrier density, Schottky barrier height, contact resistance, and film resistivity). I-V data were obtained in this study initially by contacting two probes to the separated metal contacts, and varying the applied voltage while measuring the corresponding current response.

For later studies, sensors were mounted on the sensor platform shown in Figure 3.21.The sensor platform included the sensor mounted on a 16 pin transistor outline (TO) header using double sided capton tape or silver paint. Electrical connections from the sensor to the header were done by wire bonding and I-V data was obtained by two point probe measurements. 
Depending on the choice of contact metal and the semiconductor/contact interface, the I-V response can be linear (Ohmic behavior) or non-linear (Schottky behavior). The resistance is constant and equal to the inverse of the slope of the I-V curve, when the behavior is Ohmic. When the behavior is Schottky, the slope is taken at high voltage above the point where the Schottky barrier has been overcome and the dependence is once again linear.

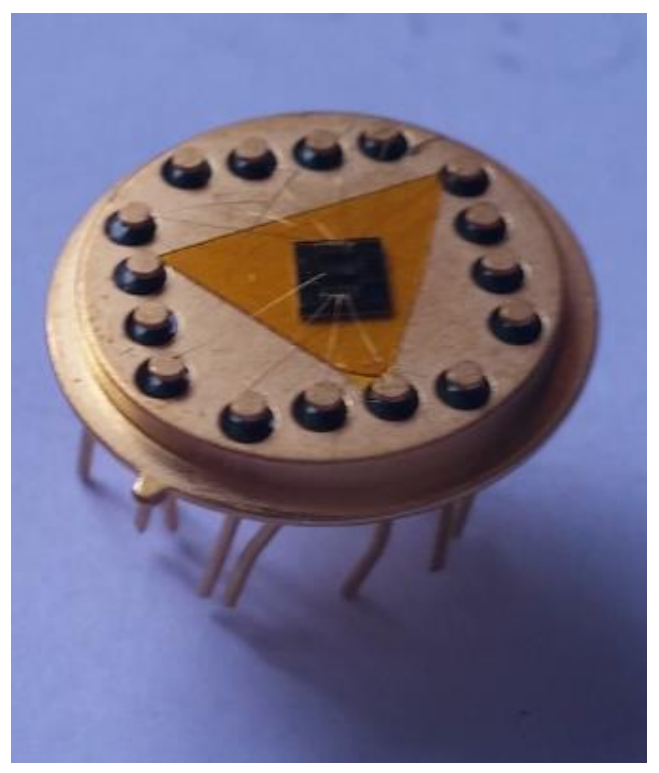

Figure 3.21: Sensor platform for electrical measurement. 


\section{Chapter 4 RESULTS AND DISCUSSION}

The first step of the research was to optimize synthesis of graphene to obtain a desired number of graphene layers using a low temperature surface chemical route based on halogen plasma-etching chemistries. The process involved two steps -1 ) etching of the $\mathrm{SiC}$ surface using $\mathrm{CF}_{4}$ to selectively etch $\mathrm{Si}$ atoms from the surface while leaving a carbon-rich layer, and 2) thermal annealing of the samples to remove volatile halogens species and reconstruct the surface for graphene growth. All the important parameters from both the steps are investigated to obtain high-quality graphene. The films were characterized using XPS, RHEED, Raman spectroscopy and AFM throughout. This chapter provides a detailed discussion of the results.

\subsection{Graphene Synthesis}

As described earlier, the synthesis process consists of two steps, etching and annealing. Both these steps need to be optimized for reproducible results.

\subsubsection{Synthesis Using Ultra High Vacuum Annealing}

The Triton Phantom III series ICP-RIE was used for etching the samples with a $\mathrm{CF}_{4}$ plasma. The ICP power primarily controlled the density of ions in the plasma, while RIE power controlled the energy (bias voltage) at which the ions impact the surface. In these studies, the ICP power was maintained at $600 \mathrm{~W}$, while the RIE power was varied to investigate its effect on the graphene thickness. After etching, the samples were annealed at $950^{\circ} \mathrm{C}$ in the UHV system.

Studies were performed in order to optimize the time for the etching. It is important to note that the etching process was divided into multiple cycles, rather than a single continuous etch. This prevented heating due to long exposure to the plasma, which can affect graphene quality and damage to the ICP-RIE system. Figure 4.1 shows a profile of the substrate surface obtained by masking a portion of the surface and etching for 3 minutes. The plasma conditions were $20 \mathrm{sccm}$ of $\mathrm{CF}_{4}, 25$ mTorr of pressure, ICP power of $600 \mathrm{~W}$, and RIE power of $300 \mathrm{~W}$. Figure 4.2 shows the similar profile after four three minutes cycles. From these profiles, it can be seen that the nominal etch rate of $200 \mathrm{~nm} / \mathrm{min}$ remained constant from cycle to cycle over the 12 minute etch period. Moreover, with the exception of side wall effects (i.e. tapering near the sidewall), etch is uniform over the exposed area. XPS analysis (described later) revealed that both the 3 minute and the 12 minute etch produce the same number of graphene layers. 


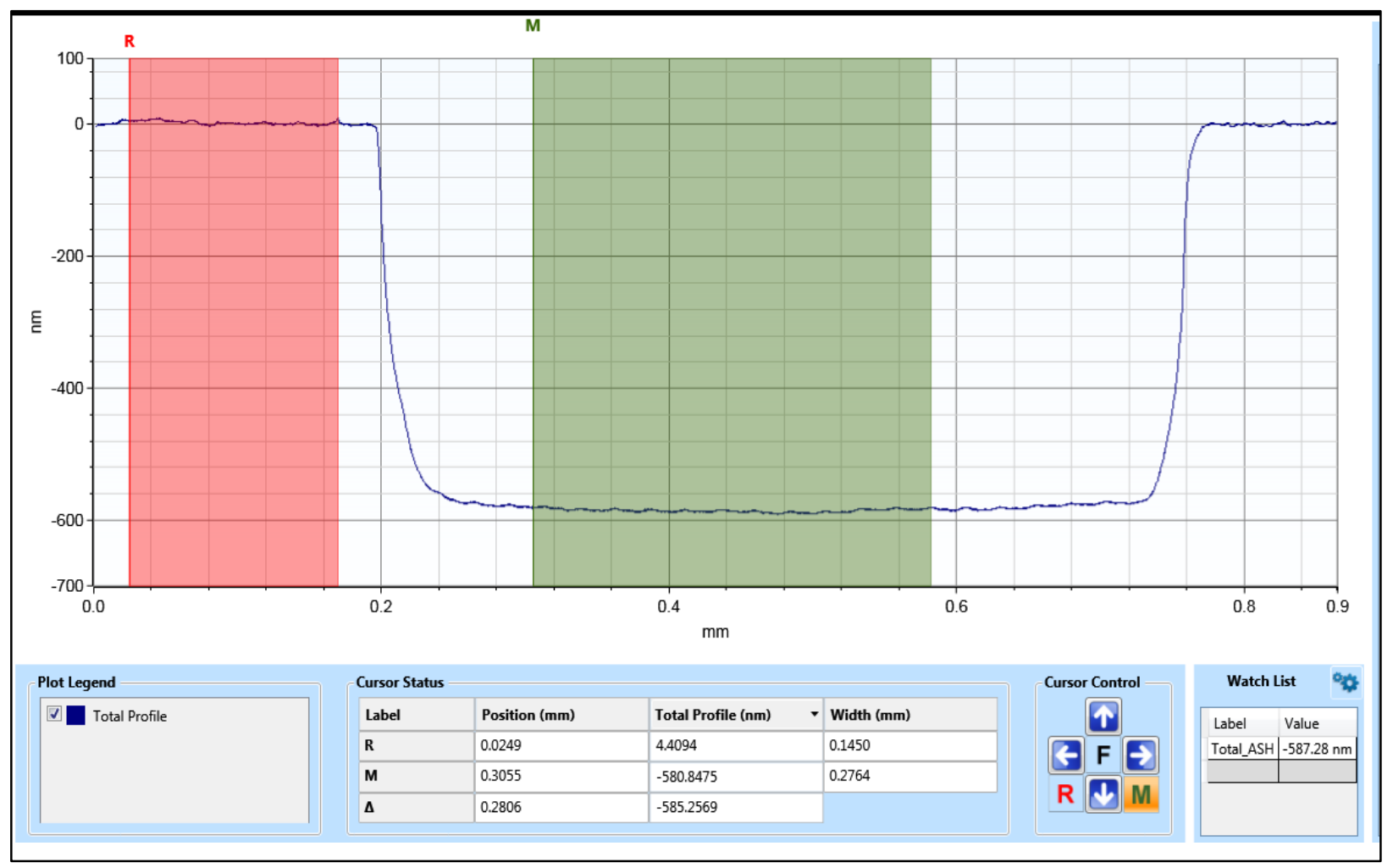

Figure 4.1: Profile of the SiC surface showing change in height after one 3 minute $\mathrm{CF}_{4}$ etch cycle.

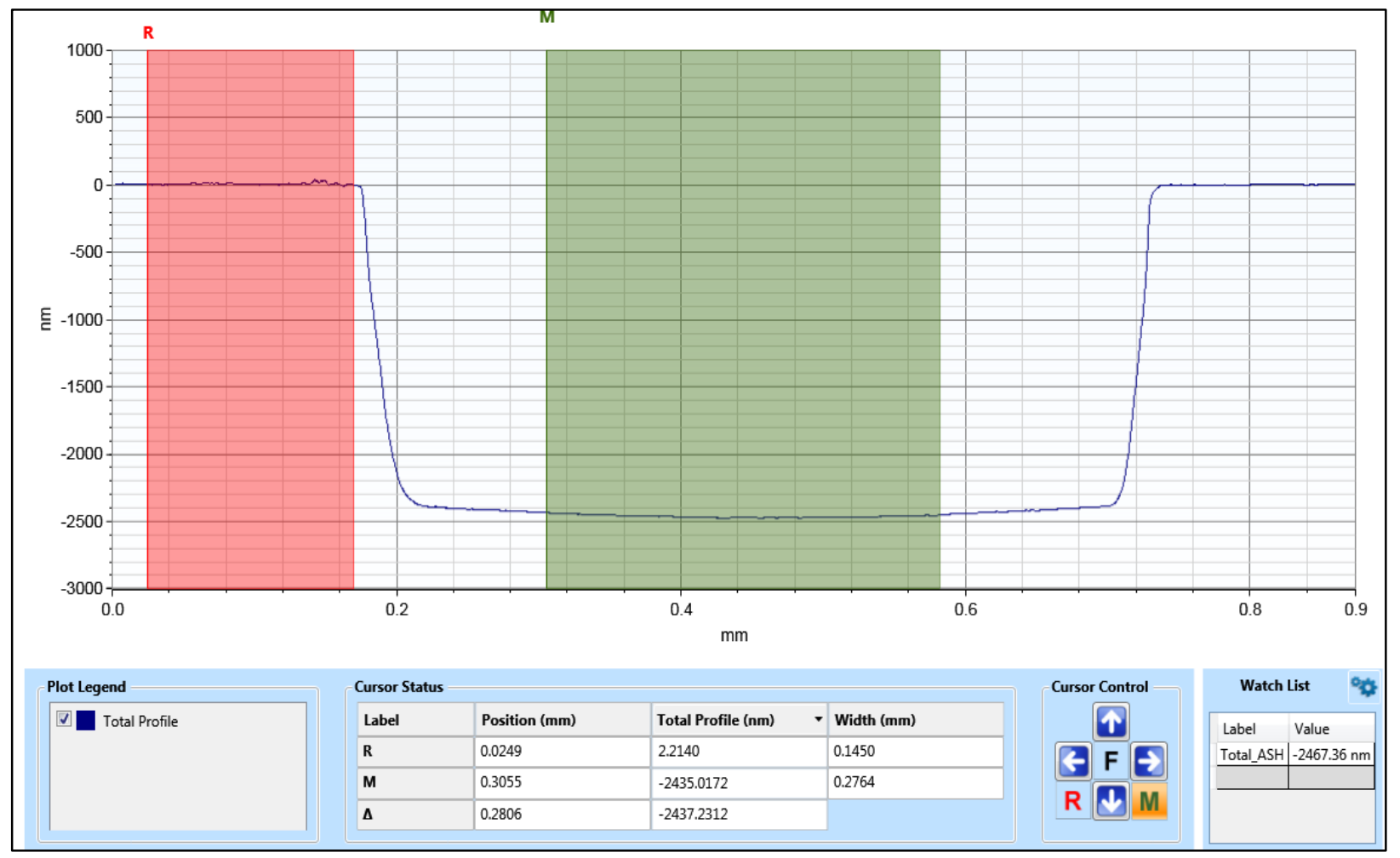

Figure 4.2: Profile of the SiC surface showing change in height after four 3 minutes of $\mathrm{CF}_{4}$ etch cycles. 
However, the carbon-oxygen ratio was lower after the 12 minute etch. This reflects the fact that even though the chamber was pre-cleaned by an $\mathrm{O}_{2}$ plasma step and preconditioned by a $\mathrm{CF}_{4}$ plasma step, chamber conditioning continued throughout the etch process. This is one of the drawbacks of working in a shared research facility. Once the samples were etched, they were annealed under UHV conditions.

Figure 4.3 show the survey spectra for the $6 \mathrm{H}$ - SiC (0001) surface before etching, after etching and after annealing. The spectra have been normalized to yield the same C1s intensity. Before etching, the ratio of $\mathrm{C}$ to $\mathrm{Si}$ is nearly 1:1 after taking into account elemental sensitivity factors. After etching and annealing, silicon has been depleted with respect to carbon and ratio of $\mathrm{C} / \mathrm{Si}$ has increased to $3: 1$, as seen in Figure 4.3. This clearly demonstrate the selective etching of silicon relative to carbon.

Figure 4.4 shows a high resolution $\mathrm{C} 1 \mathrm{~s}$ spectra for the $6 \mathrm{H}-\mathrm{SiC}$ surface etched at three different RIE power levels. This spectrum can be resolved into four components, the peak at $282.5 \mathrm{eV}$ is attributed to C-atoms bound on SiC. While the peak as $284.1 \mathrm{eV}$ is identified as graphene. The minor peaks at $285.7 \mathrm{eV}$ and $288.1 \mathrm{eV}$ can be attributed to the $\mathrm{C}_{\mathrm{C}}$ and $\mathrm{C}_{\mathrm{F}}$ defect species. The presence of these defect species causes the graphene to buckle [46]. These defects are illustrated in Figure 4.5. Recently, Duan et al. performed density functional calculations for a computational modelling of these defects [97]. Their results indicated that the paired $C_{F}$ defects are the most probable based on the minimization of surface energy.

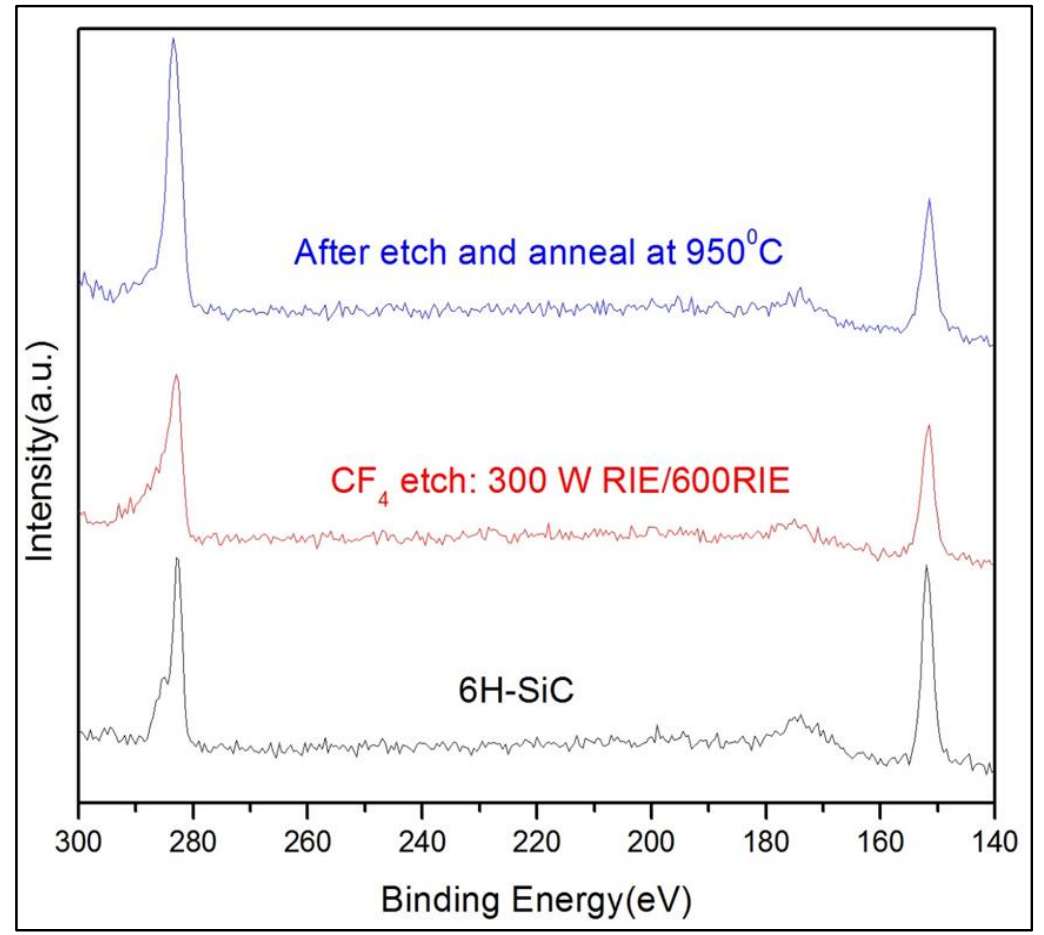

Figure 4.3 :Si2s and $\mathrm{C} 1$ s peak for $6 \mathrm{H}-\mathrm{SiC}$, after the $\mathrm{CF}_{4}$ etch and after annealing. 


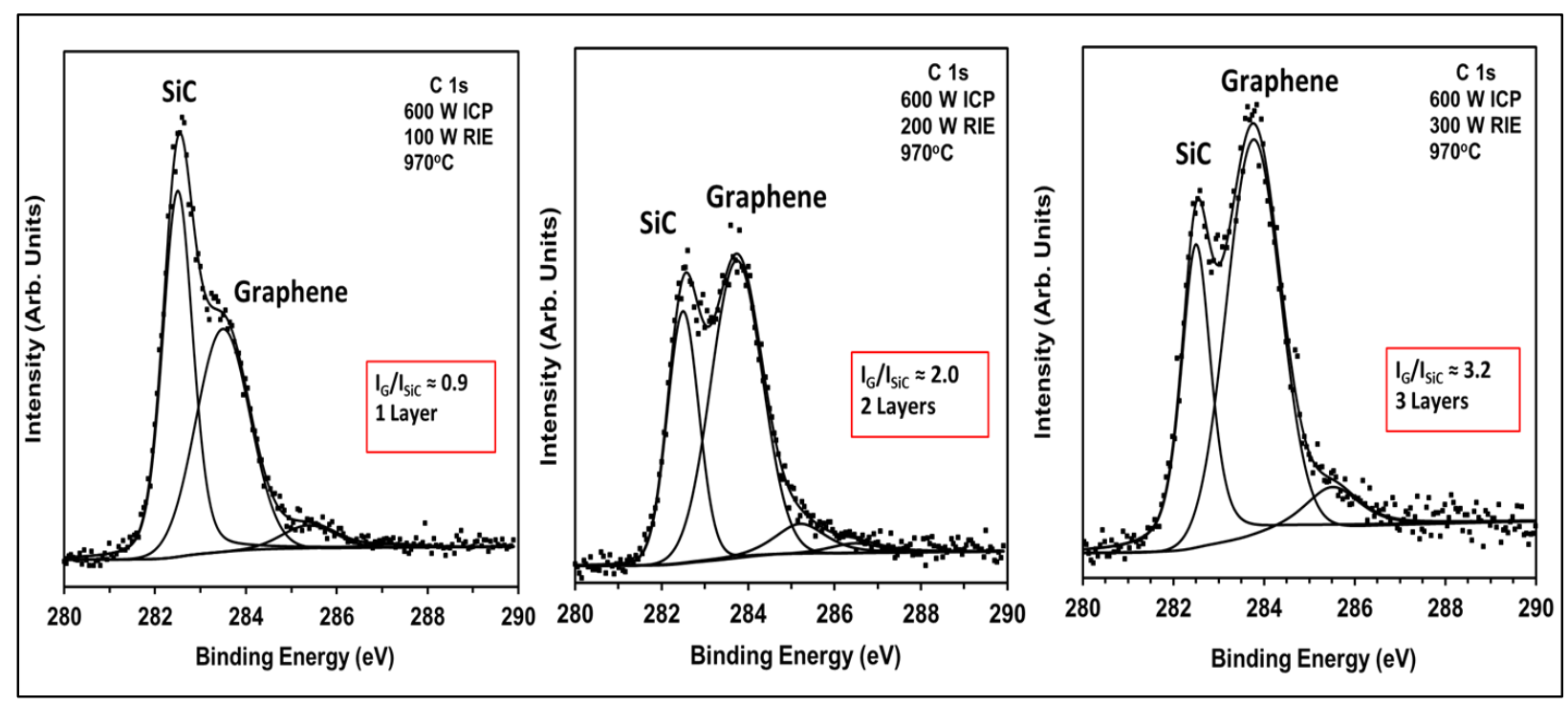

Figure 4.4:C1s Spectra of showing the effect of RIE power on number of layers for UHVA graphene samples.

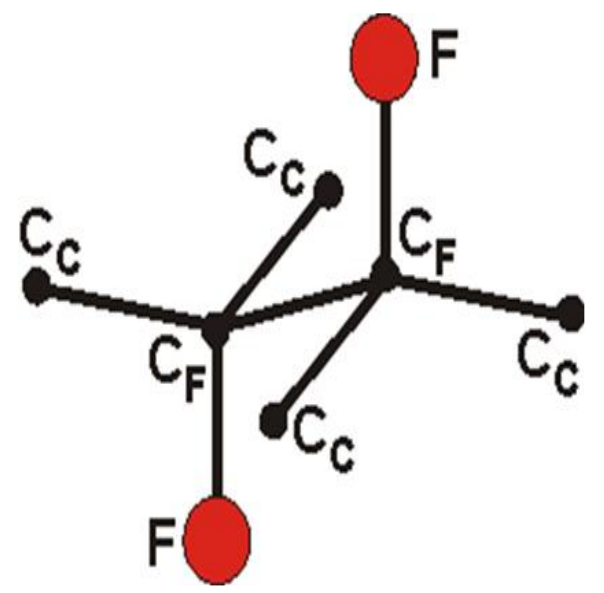

Figure 4.5: Schematic of fluorine- based defects in graphene films.

Based on the ratio of the graphene and defect peak intensities to the SiC peak intensity, the thickness of each film has been calculated. In Figure 4.6(a), film thickness is plotted as a function of RIE power. Figure 4.6(b) shows the relationship between bias voltage and RIE power. Clearly the film thickness is linearly dependent on both parameters. It is evident from these results that the energy of the incident ion (as reflected by RIE power and bias voltage) plays a crucial role in controlling film thickness. A simple model of the plasma etching suggests that as preferential etching produces a carbon-rich damage layer that rapidly reaches steady-state thickness and this thickness is controlled by the applied bias voltage. As 
mentioned above, the defects which are due to chemically bonded $F$ atoms can be used to modify properties like electrical resistivity and carrier density of graphene.

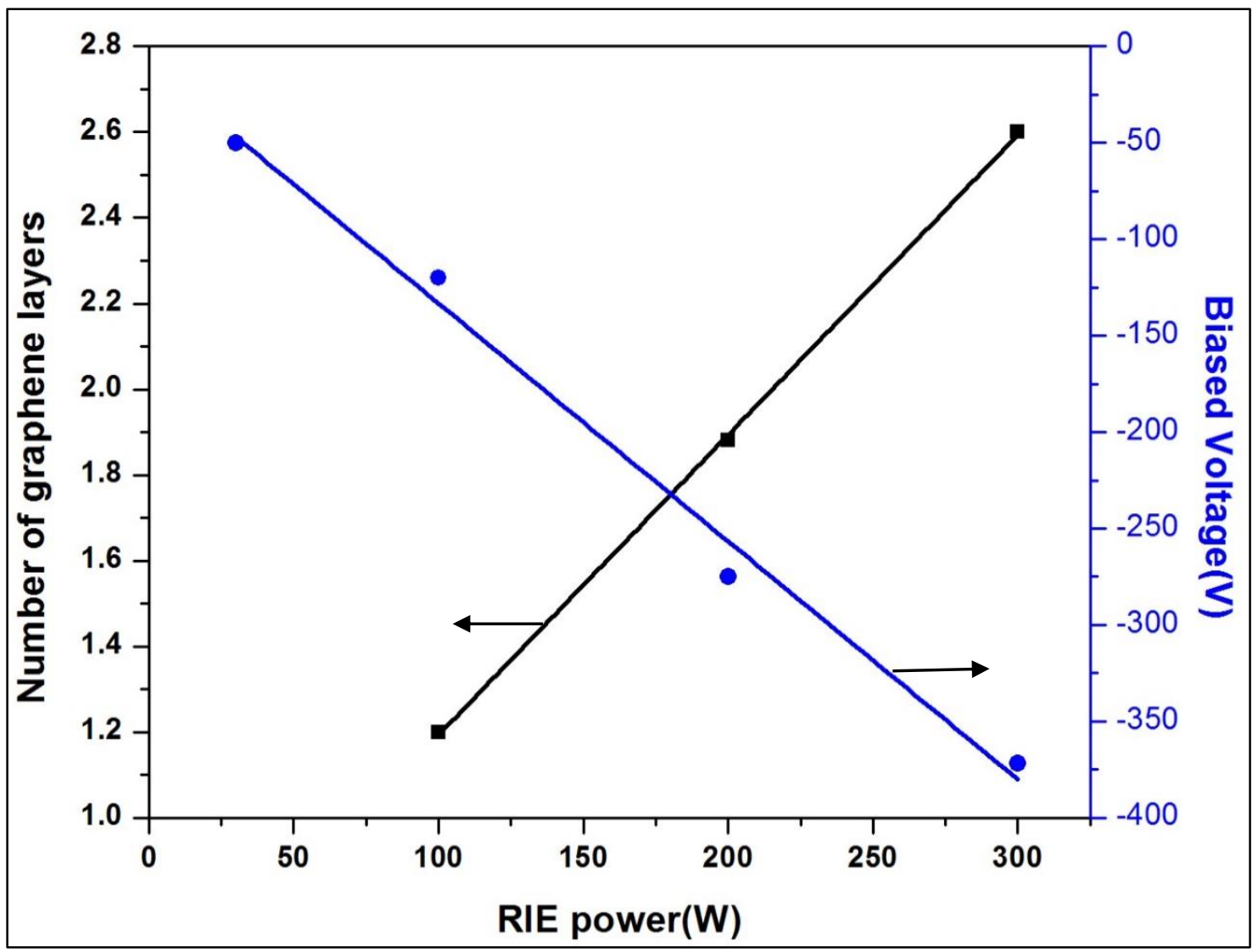

Figure 4.6: Plot of biased voltage Vs. RIE power and corresponding plot of graphene thickness versus RIE power for $\mathrm{CF}_{4}$ plasma for UHVA samples.

Figure 4.7 (a) shows the F 1s XPS spectrum for a single layer film. This peak consists of two components. One is located at $685.6 \mathrm{eV}$ and the other at $687.6 \mathrm{eV}$. Both peaks have a FWHM of $1.7 \mathrm{eV}$. The presence of two peaks is consistent with the picture of the halogenated defect states consisting of atoms bound both above and below the surface plane. The lower binding energy peak is associated with the subsurface F-atoms. This assignment is motivated by that fact that the highly electronegative F-atom can extract charge from C-atoms in the graphene film as well as C- and / or Si-atoms at the surface of the substrate. This additional electronic charge in the valence level of the F-atom provides shielding of the $F$ 1s level from the positive nuclear core and, thereby, reduces the F 1 s binding energy relative to those $\mathrm{F}$ atoms adsorbed above the surface, which can extract electrons only from the $\mathrm{C}$-atoms in the graphene film. Given this assignment one would expect the intensity of the higher binding energy peak to be greater than that of the subsurface $F$ 1s peak. The fact that the two intensities are approximately the same, 
suggests that somewhat more of the surface F-atoms are desorbed than their subsurface counterparts during the annealing process. This is consistent with the relative intensities of the $C_{C}$ and $C_{F}$ peaks in Figure 4.4.

Figure 4.7 (b) shows the F 1s spectra for a three layer film. As with the previous spectra the FWHM is $1.7 \mathrm{eV}$. Here, as observed, one expects the number of interior or subsurface F-atoms to increase relative the number of surface F-atoms, which should stay approximately the same. Figure 4.7 (c) shows the F 1s spectrum for a five-layer graphene film after the adsorption of 4-nitrophenyl diazonium. This molecule is shown in the inset. In this case, the lower binding energy peak is shifted slightly to a lower energy and

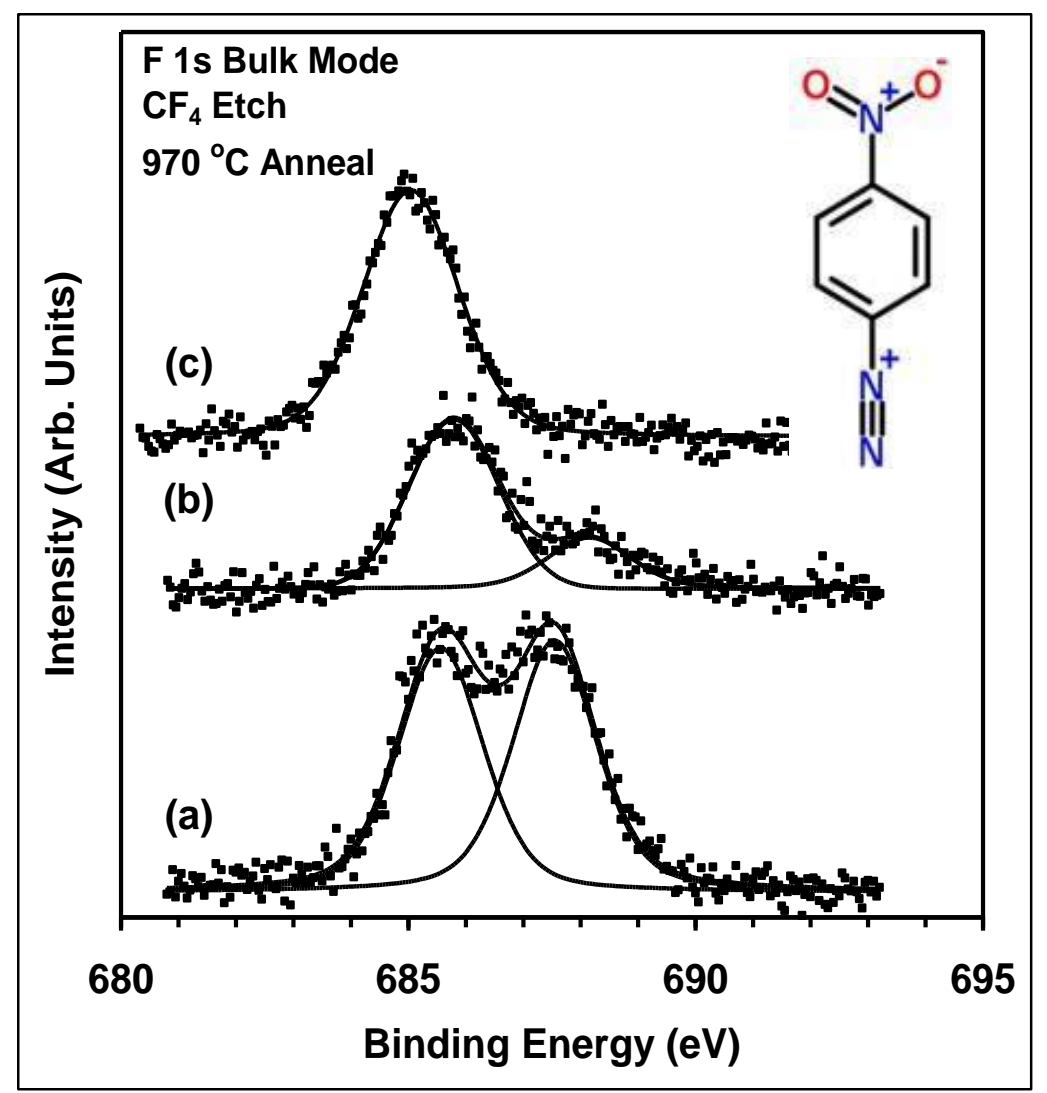

Figure 4.7: F 1s XPS spectra for single (a) and three layer (b) graphene films produced by $\mathrm{CF}_{4}$ based ICP-RIE and annealing at $970^{\circ} \mathrm{C}$, and (c) a similarly produced five layer graphene after reaction with diazonium. The nominal FWHM of all peaks is $2.0 \mathrm{eV}$.

the higher binding energy peak is not observed. This suggests that the surface fluorine has been desorbed in the reaction. This may involve the formation of volatile NF species. The point to be made here, however, is that this observation further supports the assignment of the higher binding energy $F$ 1s peak as the surface species, since it is not likely that the surface reaction would involve the subsurface F-atoms. 
Figure 4.8 shows a RHEED pattern obtained with the electron beam along the $[1 \overline{1} 00]$ of the $6 \mathrm{H}$ $\mathrm{SiC}$ substrate before and after graphene growth. Figure 4.8(a) shows the $\mathrm{SiC}$ before grapheme formation, whereas the vertical lines in Figure 4.8(b) show the $0^{\text {th }}$ and $1^{\text {st }}$ order graphene features, while the arrows indicate features from the underlying SiC substrate. This pattern is consistent with a graphene film that is rotated by $30^{\circ}$ relative to the substrate. This pattern is quite similar in orientation to that shown by Feenstra and coworkers for graphene grown by UHV annealing of the SiC (0001) surface [96]. Based on an analysis of the rotated reciprocal lattices, the fractional spacing of the graphene and $6 \mathrm{H}-\mathrm{SiC}$ features is $a_{G} /\left(a_{s i c} \sqrt{ } 3\right)$, while the measured spacing is 0.49 . This yields a value of $2.61 \mathrm{~nm}$ for $a_{G}$ if it is assumed that $a_{\text {sic }}$ is $3.08 \mathrm{~nm}$. When compared with the nominal value of $2.46 \mathrm{~nm}$ for $\mathrm{a}_{\mathrm{G}}$, this shows there is approximately $6 \%$ tensile strain in the graphene films grown here. This may seem large; however it should be noted that graphene has approximately a $20 \%$ lattice mismatch relative to $6 \mathrm{H}-\mathrm{SiC}$. The source of the $6 \%$ strain in the present case may be the buckling of the surface due to the halogen- and oxygen-based defects which are present in the film. The diffuse, spotty nature of the RHEED features may also be due

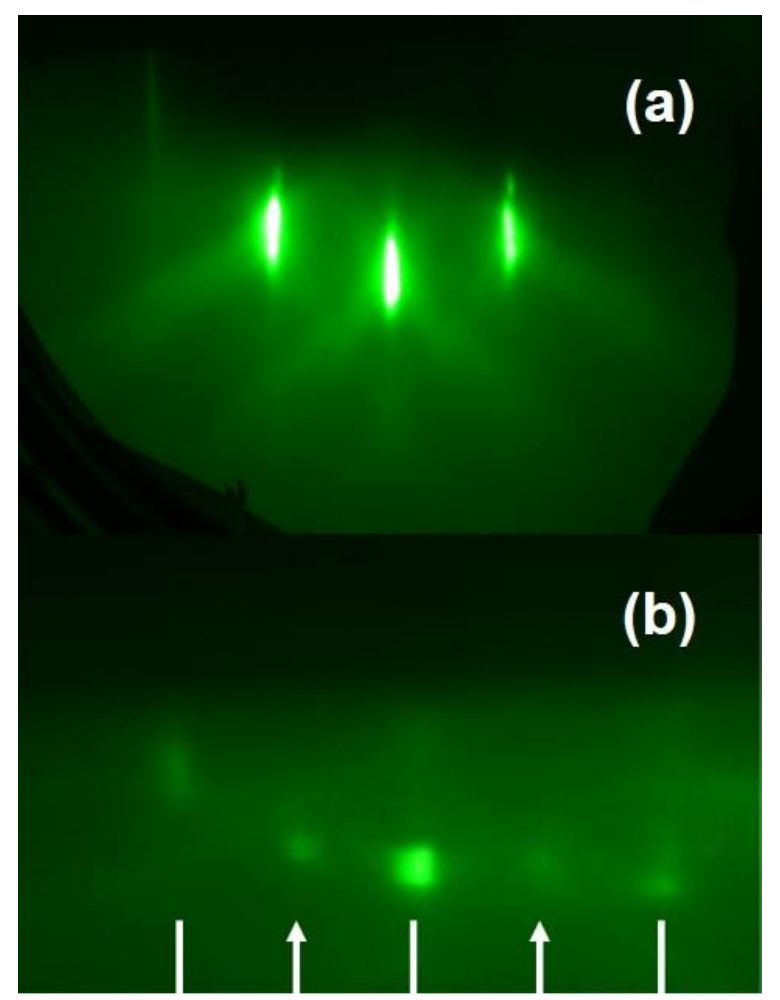

Figure 4.8: RHEED patterns obtained a) before and b) after graphene synthesis.

to this buckling of the surface as is the surface roughness of the films.

Figures 4.9 (a) and (b) show Raman spectra representative of the SiC substrate and a two-layer graphene film, respectively. A comparison of the spectra (a) before and (b) after growth shows that the 
graphene film attenuates the SiC spectrum by $\sim 30 \%$. Figure 4.9 (c), the difference spectrum, was obtained by scaling the SiC spectrum, subtracting it from the spectrum for the film, and performing a baseline operation. From Figure 4.9 (c), it can be seen that the Raman peaks associated with the film are located at $1352 \mathrm{~cm}^{-1}\left(76 \mathrm{~cm}^{-1}\right), 1597 \mathrm{~cm}^{-1}\left(63 \mathrm{~cm}^{-1}\right), 2689 \mathrm{~cm}^{-1}\left(120 \mathrm{~cm}^{-1}\right)$, and $2915 \mathrm{~cm}^{-1}\left(188 \mathrm{~cm}^{-1}\right)$. Here the numbers in brackets are the corresponding FWHM values. Although there are peak shifts of up to $17 \mathrm{~cm}^{-}$ 1 , the first three peaks compare reasonably well with the $D, G$, and $2 D$ regions expected for the exfoliated graphene as well as graphene formed by sublimation of Si from SiC $[93,97]$. The FWHM values for these peaks are, however, considerably broader than those observed for exfoliated graphene or graphene formed by sublimation of Si from SiC. The feature at $2915 \mathrm{~cm}^{-1}$ is not observed for either exfoliated graphene or graphene formed by sublimation of $\mathrm{Si}$ from $\mathrm{SiC}$. The $\mathrm{D} / \mathrm{G} / 2 \mathrm{D}$ ratio for the difference spectrum is $0.5 / 1 / 0.1$. While the $D / G$ ratio is comparable to what is found for graphene formed by sublimation of

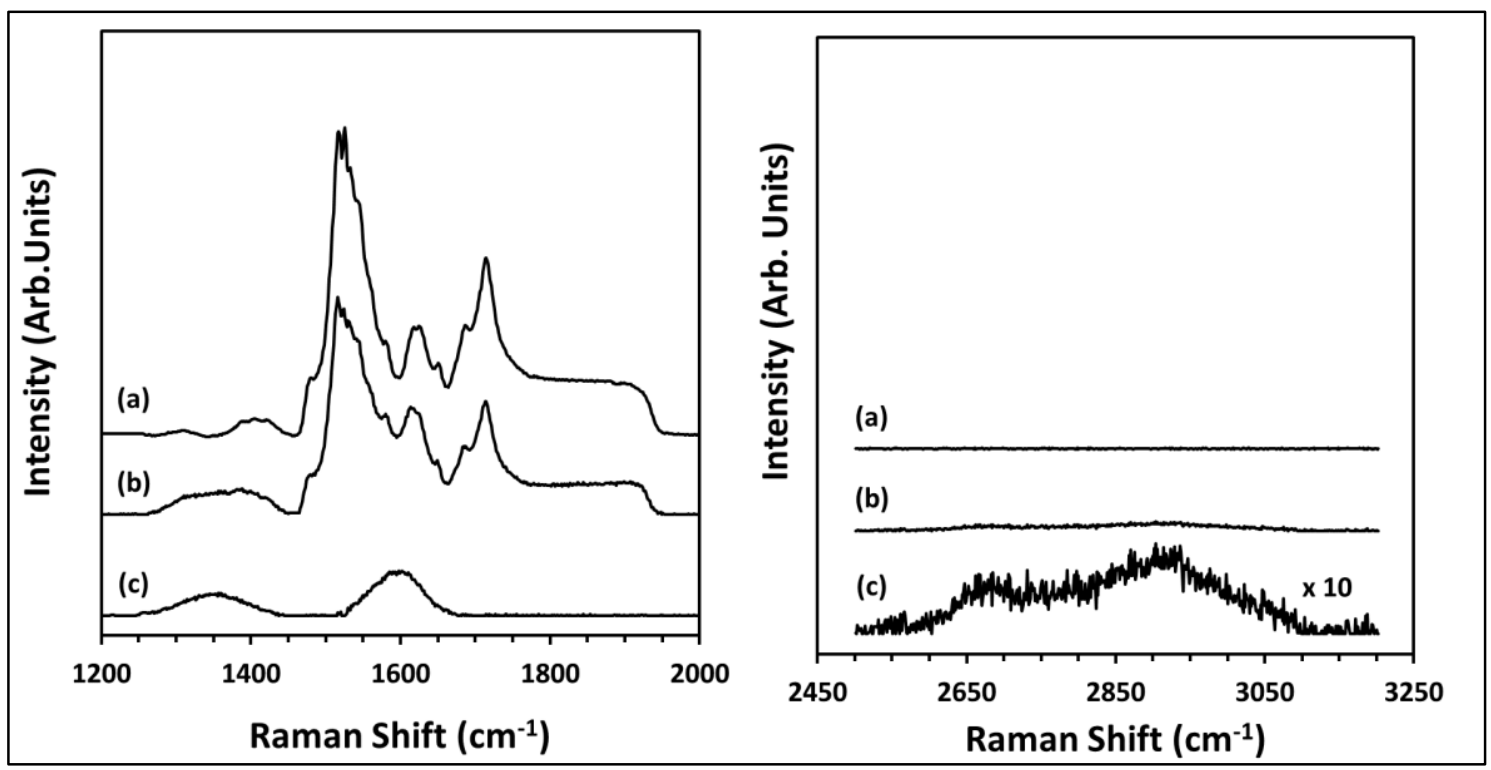

Figure 4.9: Raman spectra for a) the 6H-SiC substrate, b) a two layer graphene film, and c) the difference spectrum after scaling and subtracting the $a$ and $b$.

Si from $\mathrm{SiC}$, the $\mathrm{G} / 2 \mathrm{D}$ is not $[97,98,99]$.

Robinson et al. studied the nucleation of graphene on SiC (0001) surfaces using the sublimation process [97]. Using $488 \mathrm{~nm}$ radiation, they found that graphene nucleated at step edges on the surface and that the $D / G / 2 D$ (peak height) ratio varied from $0.05 / 1 / 0.59$ to $0.73 / 1 / 0.53$ as the annealing temperature decreased from $1425^{\circ} \mathrm{C}$ to $1225^{\circ} \mathrm{C}$. They attributed this variation to temperature dependent changes in grain size. Shivaraman et al. using $488 \mathrm{~nm}$ radiation observed a D/G/2D ratio of 0.07/1/0.73 for few-layer graphene formed above $1300^{\circ} \mathrm{C}$ [98]. Finally, Röhrl et al. using $532 \mathrm{~nm}$ radiation found a 
$\mathrm{G} / 2 \mathrm{D}$ ratio of $1 / 0.81$ for few layer graphene films formed at annealing temperatures above $1200^{\circ} \mathrm{C}$ (no information on the $D$ peak was given) [99]. Thus, the $D / G$ ratio $(0.5 / 1)$ observed in the present studies is within the range of values observed for graphene films grown on SiC (0001) surfaces by Si sublimation, but the $G / 2 D$ ratio $(1 / 0.1)$ is much less than expected. This issue along with the peak broadening and the new peak at $2915 \mathrm{~cm}^{-1}$ can be resolved by considering the effects of the fluorine- and oxygen-based defects on the Raman spectrum of graphene.

In studies of fluorination of graphene, Robinson et al. show the overall effect on the Raman spectra is to enhance the intensity of the $D$ peak and reduce the intensity of the $2 D$ peak relative to the $G$ peak as well as to broaden all the peaks [100]. In addition, they observed a new peak at $\sim 2961 \mathrm{~cm}^{-1}$. Although the spectra are not of sufficient quality to allow extraction of peak intensities (particularly for the 2D) and FWHM values, qualitatively, this is qualitatively what is observed for the Raman spectra reported here. In similar fluorination studies, Nair et al. observed comparable effects using $514 \mathrm{~nm}$ radiation [101]. FWHM values of $\sim 109 \mathrm{~cm}^{-1}$ for the $D$ and $G$ peaks and $\sim 163 \mathrm{~cm}^{-1}$ for the 2D peak, can be extracted from their spectra for graphene exposed to $\mathrm{XF}_{2}$ for 9 hours at $70^{\circ} \mathrm{C}$. The corresponding $\mathrm{D} / \mathrm{G} / 2 \mathrm{D}$ ratio was $1.3 / 1 / 0.2$. Although the details on the kinetics of these changes to the Raman spectra are limited, it is clear that both the FWHM values and the D/G/2D ratios depend on the extent of fluorination. Further, as discussed by Nair et al., partially fluorinated graphene exhibits Raman spectra that resemble those of graphene oxide." Consequently, it is highly likely that both oxygen- and fluorine-based defects play a crucial role in determining the peak position, FWHM values, and D/G/2D ratios in the present studies. It is important to note that the defect dependent broadening and reduction in the intensity of the 2D peak preclude its use in establishing the number of layers in the films produced here.

In contrast to terraced surfaces produced by high temperature Si sublimation, the graphene films produced by plasma etching and annealing were featureless $[45,44]$. AFM analyses reveal essentially a featureless surface with occasional hillocks with an overall root mean square (RMS) roughness of $0.5 \mathrm{~nm}$. This value is slightly more than twice the roughness that was observed previously for "atomically flat" terraces on $6 \mathrm{H}-\mathrm{SiC}$ produced by high-temperature hydrogen etching $(0.2 \mathrm{~nm})$ [102]. The increase in roughness may be associated with the buckling of the surface due to the fluorinated defects. 

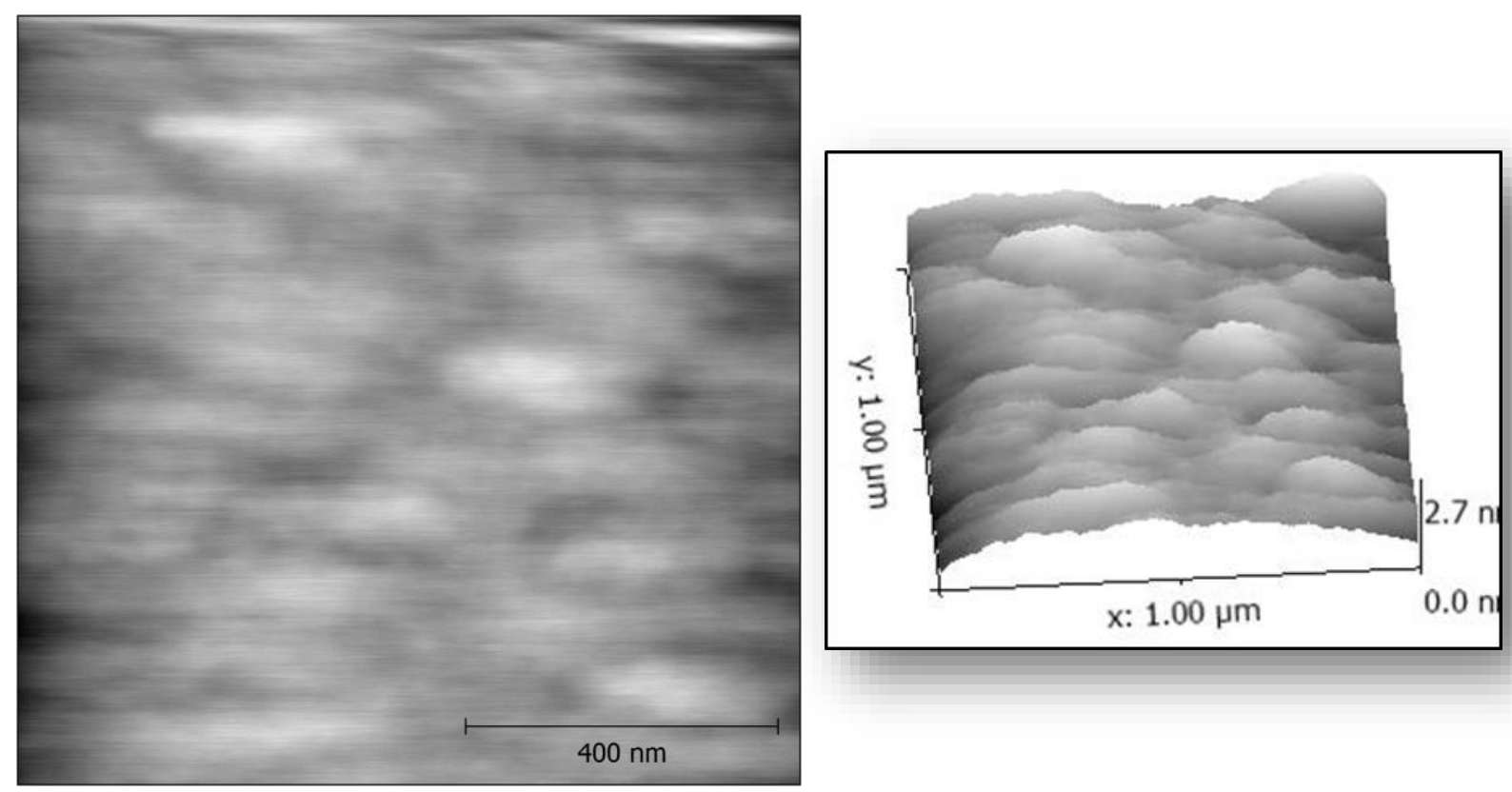

Figure 4.10: AFM image for three layer graphene, prepared by UHVA. The roughness of the surface is around $0.352 \mathrm{~nm}$.

\subsubsection{Synthesis using Rapid Thermal Annealing}

In these studies, the samples were etched in the same manner as described above, but the UHV annealing process was replaced by atmospheric pressure RTA in argon.

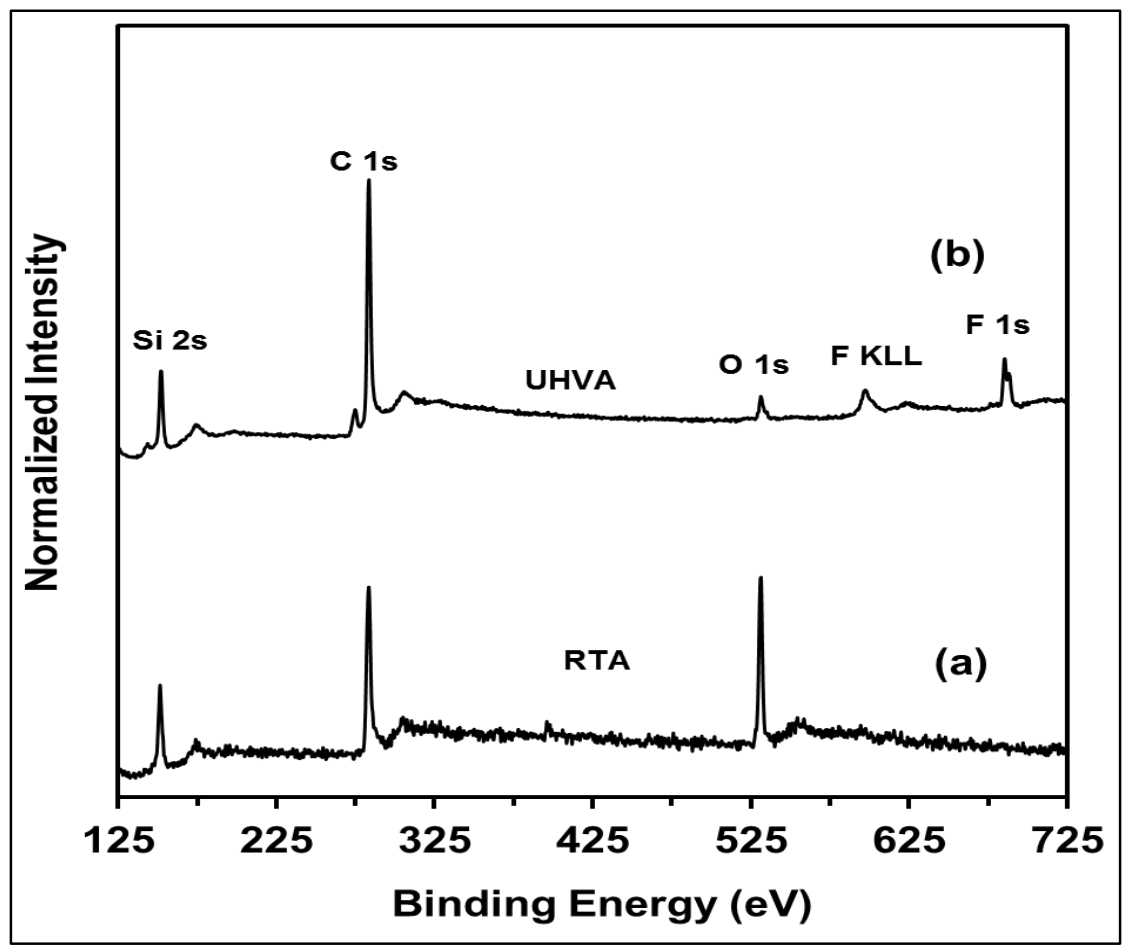

Figure 4.11: Comparison of the (a) RTA and (b) UHVA survey spectra for three layer graphene film. 
Figure 4.11 shows the survey spectra for UHVA and RTA surface after annealing at $300 \mathrm{~W}$ RIE power. Carbon, oxygen and silicon peaks are present in both the surface, however, there was no fluorine peak present on the RTA surface. Also, the intensity of the oxygen peak for RTA surface is higher than that of UHVA surface. In order to study these differences in the surface, high resolution spectra were analyzed.

Figure 4.12 shows the C1s XPS peaks for a three-layer graphene film provided by RTA. The peak at $282.5 \mathrm{eV}$ corresponds to the $\mathrm{SiC}$ substrate, while the major peak at 284.6 corresponds to the overlaying graphene film, similar to the UHV graphene. As noted above, there is no fluorine present on the surface, so the higher binding energy peaks must be due to the oxygen-based defects rather than fluorine-based defects. These defects have been seen extensively for graphene oxide. For example, Stankovich et al., found similar XPS peaks after reduction of chemically exfoliated graphene using hydrazine [103]. Based on their studies, the peak at $286 \mathrm{eV}$ corresponds to hydroxyl (C-OH) and epoxide(C-O-C) groups attached to the planar surface of the graphene, while the peak at $288.9 \mathrm{eV}$ corresponds to a mixture of carboxyl and carbonyl $(C=0)$ defects formed at the step edges of the graphene.

These assignments were further confirmed by corresponding 01s XPS spectra as shown in Figure 4.12(b). Along with the peak at $531 \mathrm{eV}$ due to $\mathrm{SiO}_{\mathrm{x}}$ or $\mathrm{SiCO}_{x}$, two additional peaks at $531.9 \mathrm{eV}$ and 533.2 $\mathrm{eV}$ are observed. These correspond to singly-bonded oxygen in hydroxyl (C-OH) and epoxide(C-O-C), or singly-bonded oxygen in carboxyls and esters, thereby confirming the presence of oxygenated defects.

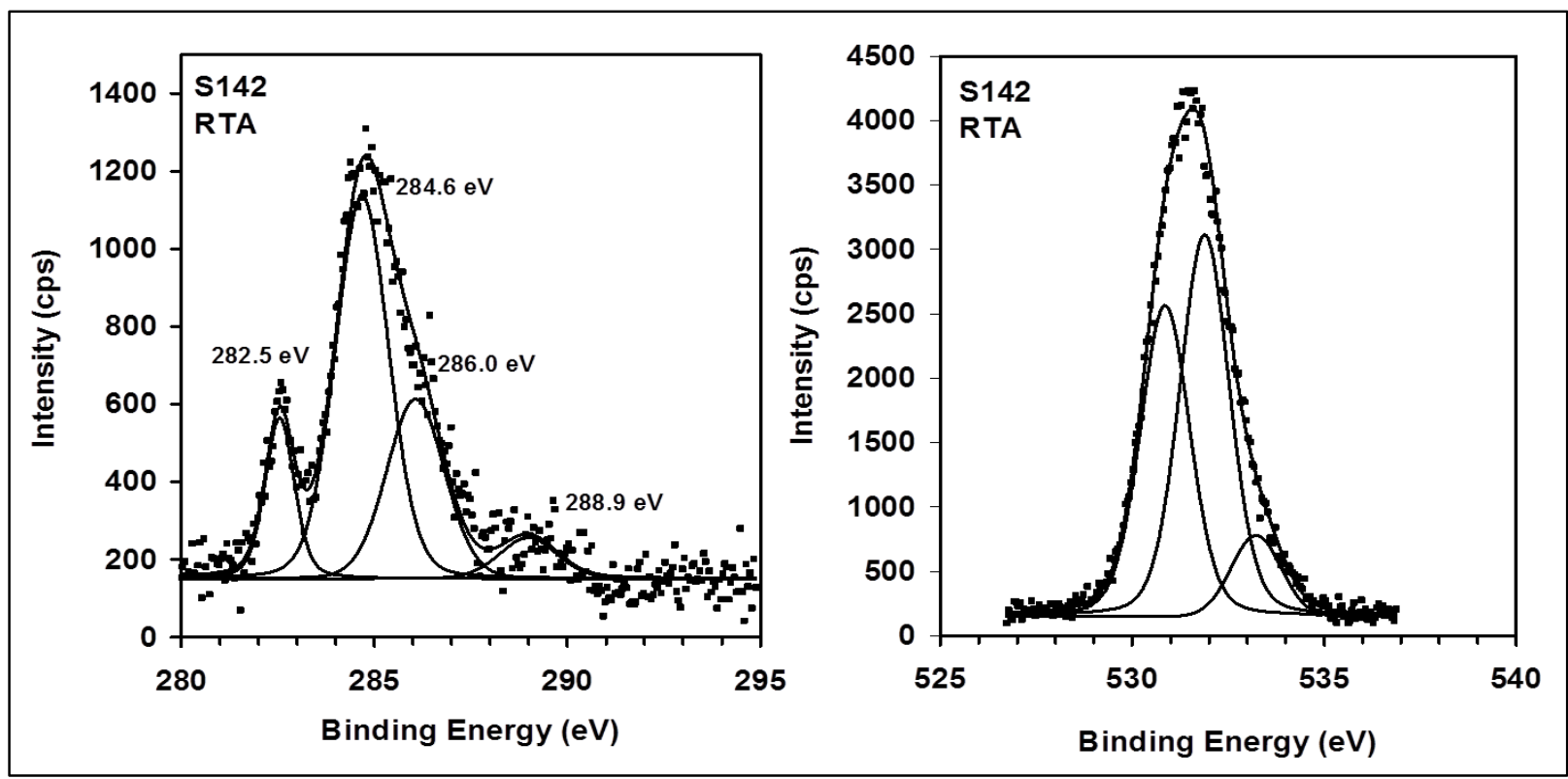

Figure 4.12:C1s spectra for three layer graphene sample (on left) and corresponding 01 s spectra (on right) after 2 minutes of rapid thermal annealing at $950^{\circ} \mathrm{C}$. 


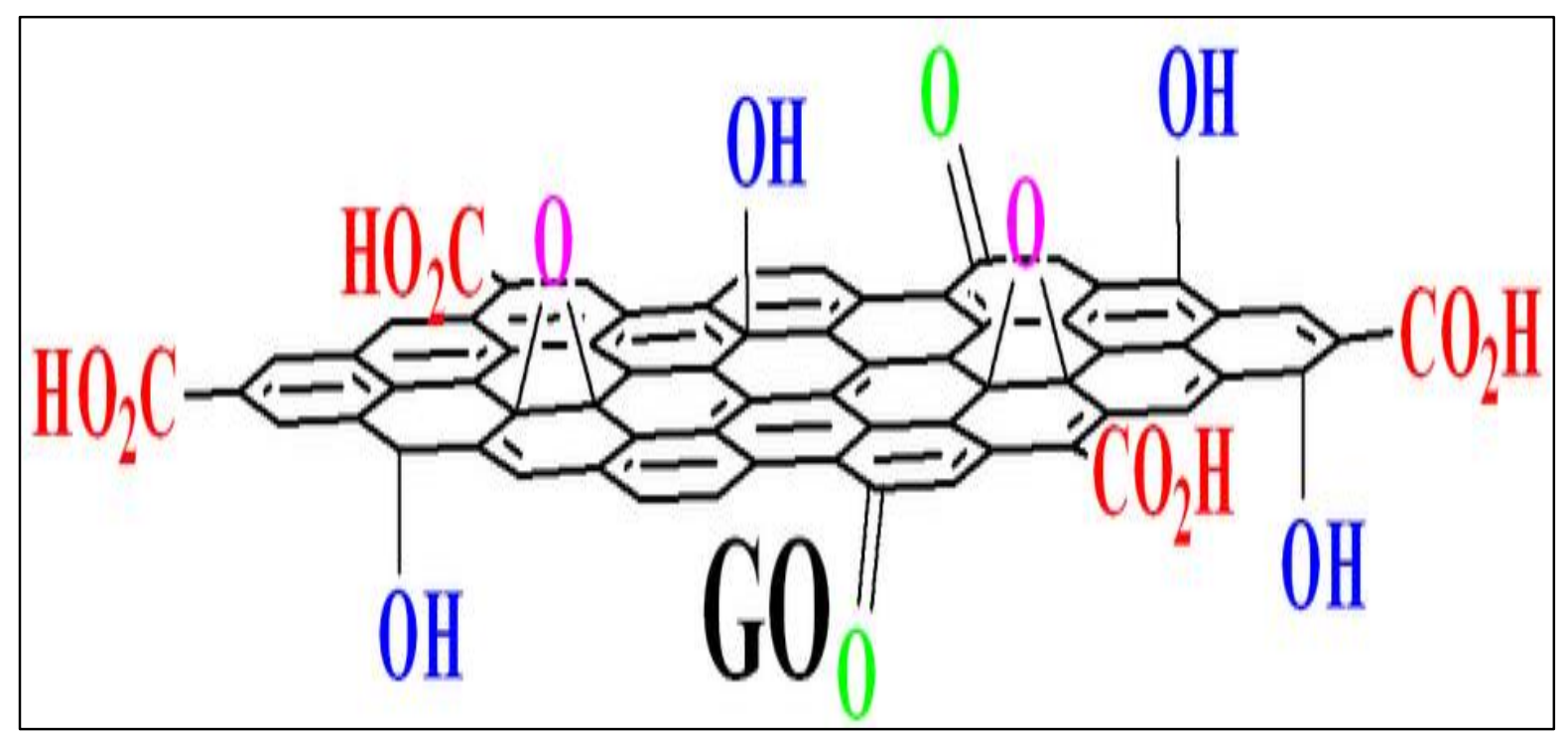

Figure 4.13: Schematic of oxygen-based defect states, as described for graphene oxide [35].

It is important to note that, the relative concentration of these defect species is much less than that observed from graphene oxide films [35]. These defects are shown in Figure 4.13. Based on the relative intensities of the defect and graphene peaks, typical defect concentrations were on the order of $36 \%$. As these oxygenated defects were different from the UHV samples, these defects need further investigation in order to understand their impact as well as their interaction for nanoparticle nucleation.

In the UHVA process, ramp rate is about $60^{\circ} \mathrm{C} / \mathrm{min}$ and the steady-state time is one hour, which is typical of most UHVA processes. In the case of RTA, the ramp rate is much faster $\left(\sim 450^{\circ} \mathrm{C} / \mathrm{min}\right)$ and the annealing time is reduced significantly because of it. Hence, it was possible to conduct a study of annealing time and its effect on the resultant film properties.

Figure 4.14 shows the oxygen concentration and film thickness vs the annealing time for a $300 \mathrm{~W}$ RIE sample. Here, it can be seen that the sample showed a four-layer film thickness and low oxygen concentration initially. But after 2 minutes, the number of graphene layers is reduced to three, which was expected for $300 \mathrm{~W}$ RIE, with an acceptable amount of oxygen concentration. Even though the 1 minute anneal resulted into the lowest concentration of oxygen, the surface of graphene was not uniform and hence, the annealing time of 2 minutes was used for all the subsequent RTA samples.

Figure 4.15 shows the graphene film thickness and bias voltage as a function of RIE power, prepared by RTA. The linearity between film thickness and RIE power was similar to the UHVA samples. 


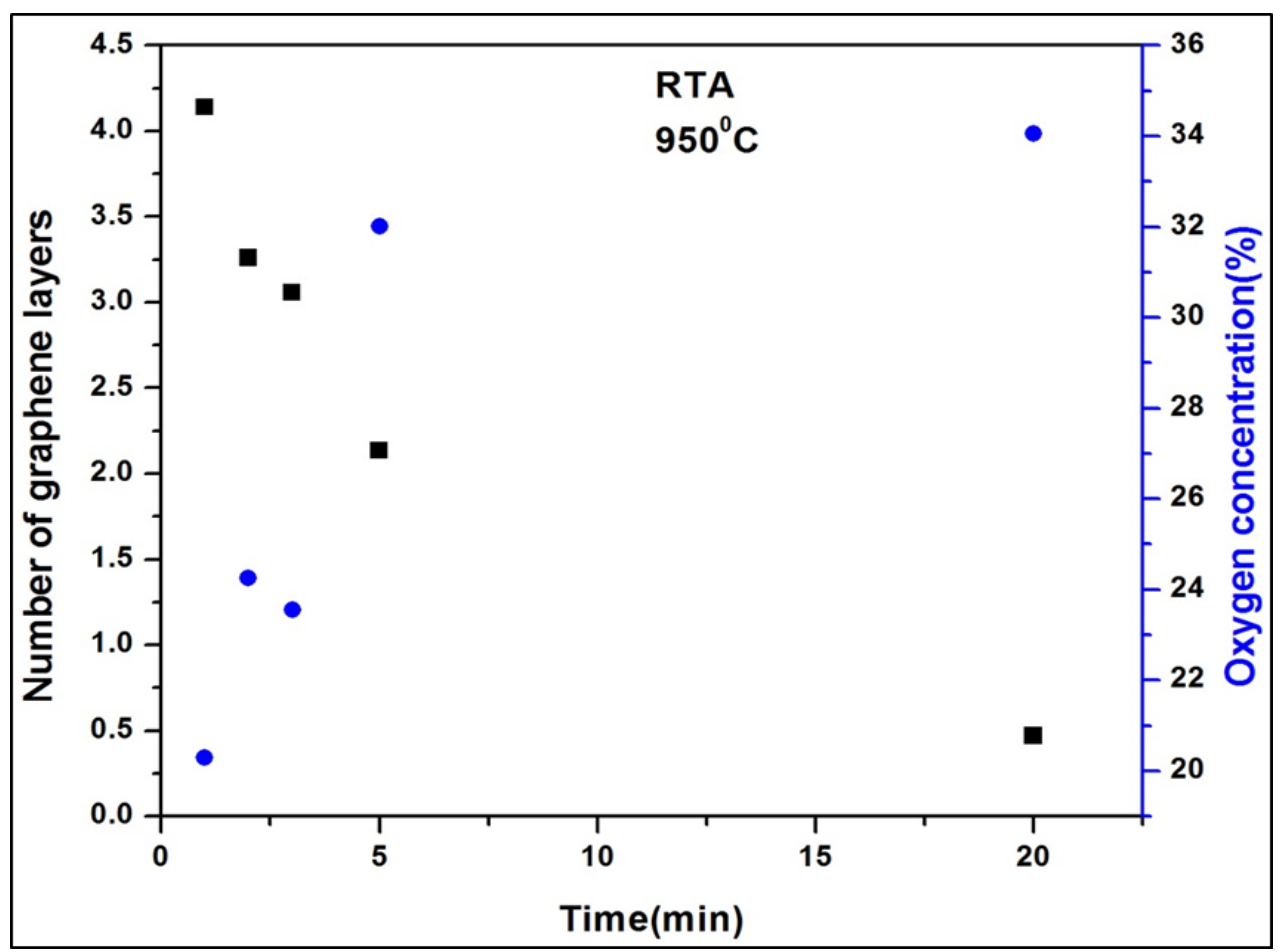

Figure 4.14: Graph showing number of graphene layers and oxygen concentration determined by XPS with respect to time for RTA samples.

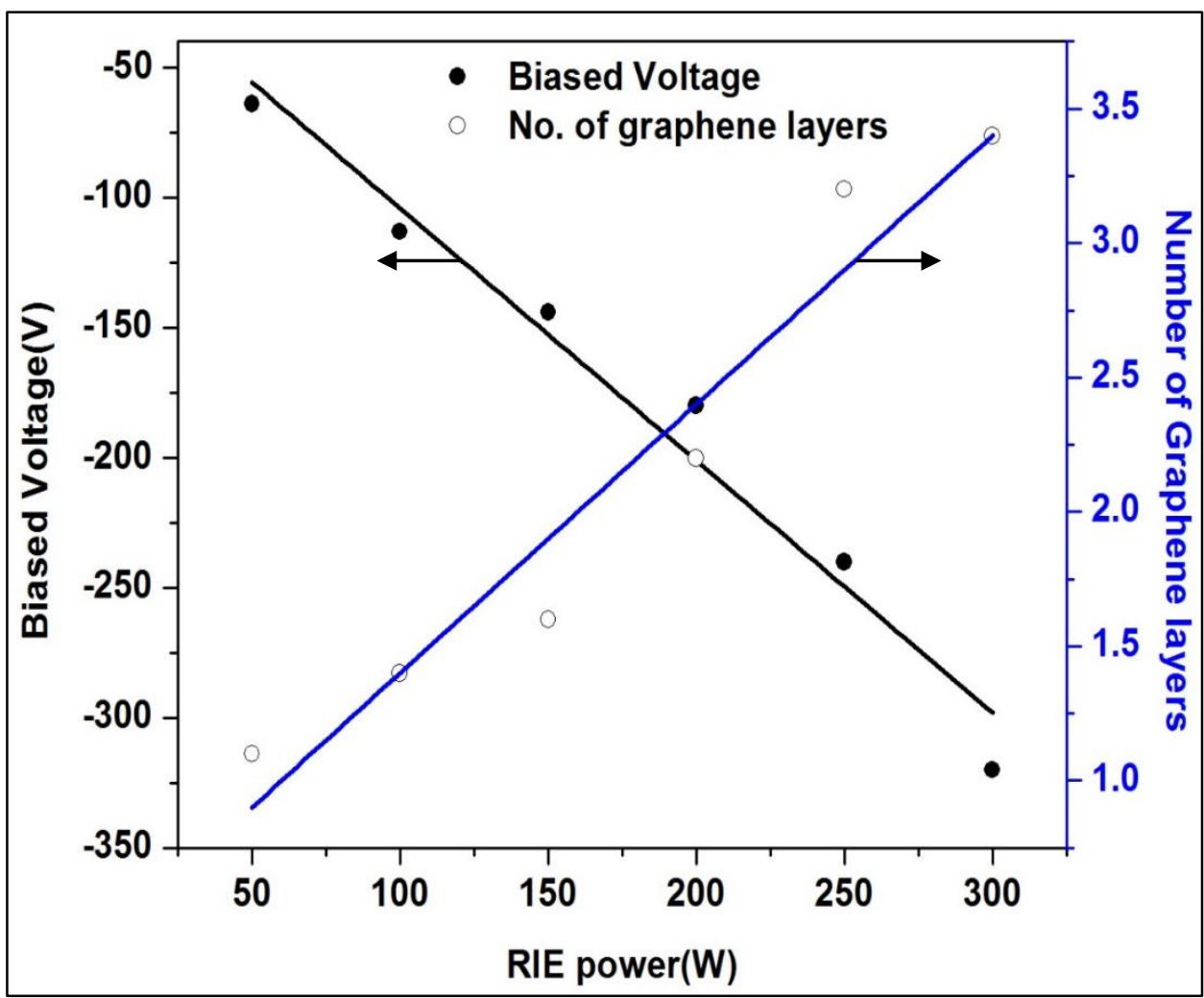

Figure 4.15: Plot of biased voltage Vs. RIE power and corresponding plot of graphene thickness versus RIE power for $\mathrm{CF}_{4}$ plasma for RTA samples. 
Figure 4.16 shows the Raman spectra for the three-layer RTA graphene sample and the Raman spectra for the $6 \mathrm{H}-\mathrm{SiC}$ surface. The $\mathrm{G}$ peak was found to be present at $1580 \mathrm{~cm}^{-1}$, along with $\mathrm{D}$ and 2D peaks at $1350 \mathrm{~cm}^{-1}$ and $2691 \mathrm{~cm}^{-1}$ respectively. These peaks are characteristic of graphene. These Raman spectra were close to those obtained by Stankovich for their oxygen defects, thus confirming high quality graphene.
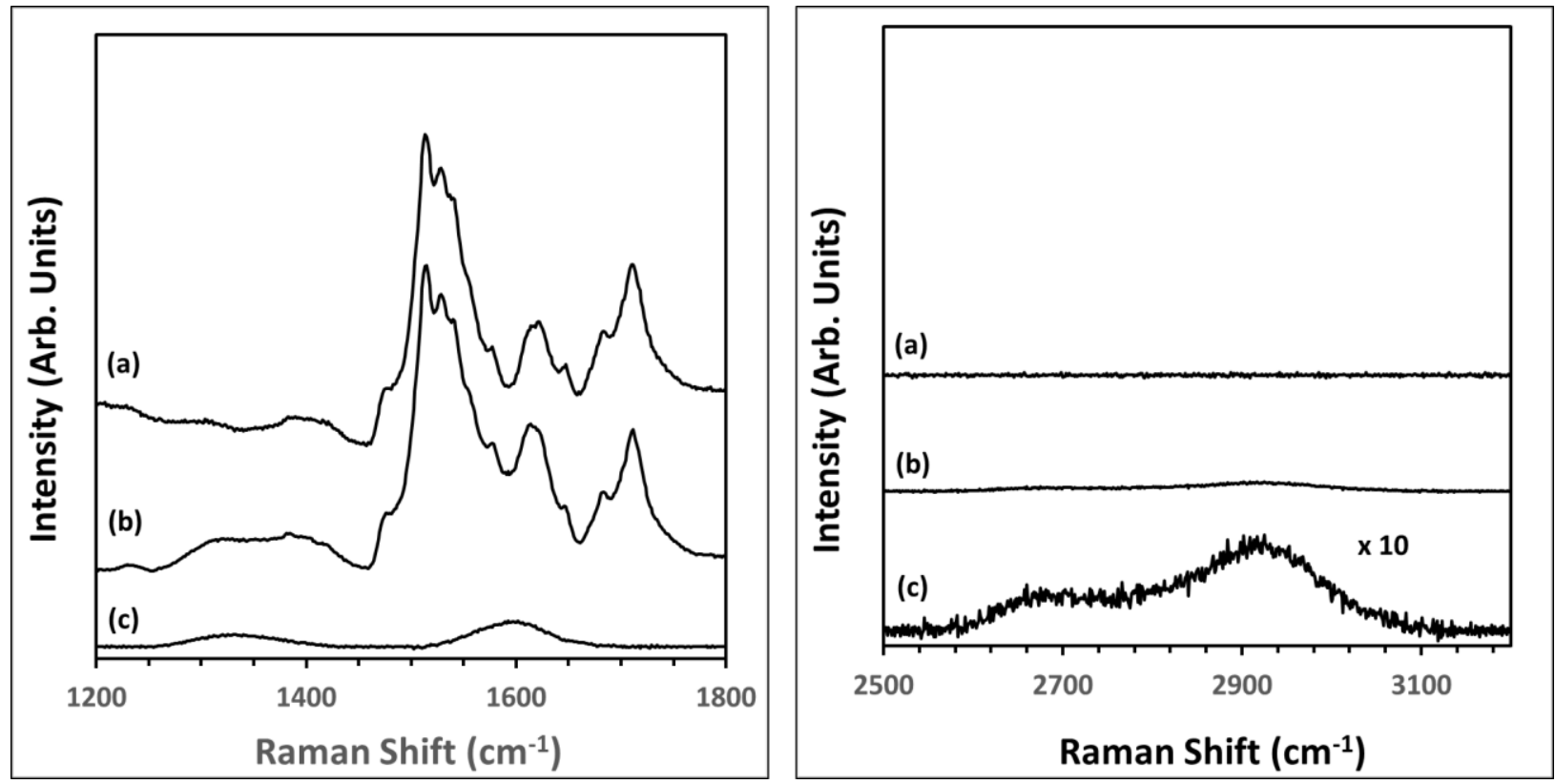

Figure 4.16:Raman spectra for a) the 6H-SiC substrate, b) a three layer graphene film, and c) the difference spectrum after scaling and subtracting the $a$ and $b$.
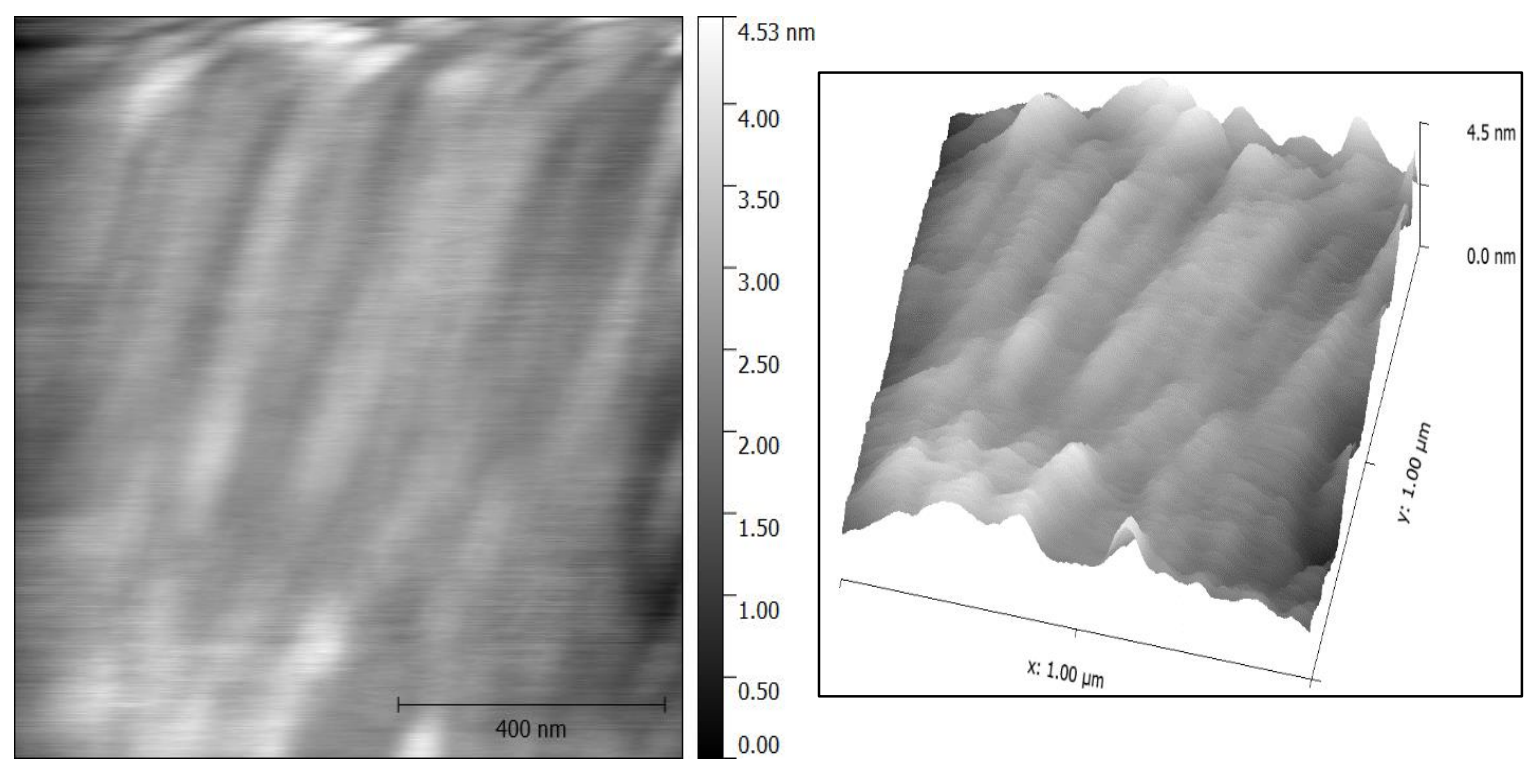

Figure 4.17: AFM image for three layer graphene, prepared by RTA. The roughness of the surface is around $0.532 \mathrm{~nm}$. 
The surface morphology of the RTA films was characterized using AFM. Figure 4.17 shows an AFM image for a three-layer graphene film. The RMS roughness of the RTA surface was typically in the range of $0.7 \mathrm{~nm}$. This is higher than the RMS roughness of the UHVA films. Here it can be seen that the low level hillocks associated with the UHVA are replaced with a ridge-like structure.

\subsection{Surface Modification}

At this point, the basic processes (UHVA and RTA) for reliably producing large area $(1 \mathrm{~cm} \times 1 \mathrm{~cm})$ graphene films of controlled thickness have been established. The next step involves modification of the defect levels. These defects may influence gas adsorption (i.e. Impact sensor sensitivity and selectivity) under certain conditions. It is also understood that defects act as nucleation sites for nanoparticles. Consequently, chemical processes that can alter the defect density should be clearly understood. In addition, the nanoparticle nucleation process involves a variety of solution chemistries. As will be seen, the studies reported in this section will form a basis for understanding these later results.

A method widely used to control oxygenated defects on graphene oxide is the reduction of graphene, which can be carried out at room temperature or at moderate temperatures. Thus, the requirements of equipment and environment are not as critical as compared with thermal reduction methods. As all these reduction methods involves water as the solvent, control experiments using deionized water for 12 hours were done to understand the effect of the solvent itself. The XPS shows negligible changes in the peaks, eliminating any contribution of the water during the subsequent surface modification studies.

Metal hydrides such as sodium hydride and sodium borohydride $\left(\mathrm{NaBH}_{4}\right)$ have been used as strong reducing agents for graphene itself [104]. $\mathrm{NaBH}_{4}$ was found to be more effective than hydrazine as a reductant for $\mathrm{GO}$. Although $\mathrm{NaBH}_{4}$ is hydrolyzed by water, the kinetics of the graphene oxide reduction is rapid enough to effectively reduce graphene. Thus, $\mathrm{NaBH}_{4}$ can be an effective reducing agent. Figure 4.18 shows the XPS C1s spectra for graphene after 12 hour of $\mathrm{NaBH}_{4}$ reduction. Relative to the initial surface, the intensity of the SiC substrate and defect peaks decrease, and the FWHM of the graphene peaks also decreases. The reduction of the defect peaks indicates that their concentration on the surface has decreased from $36 \%$ to $26 \%$. The narrowing of the FWHM of the graphene peak suggest that the bonding environment of the surface is less diverse. This is consistent with the removal of defect species. The reduction of the intensity of the substrate peak indicates that the overlaying film is denser after reduction. This is also consistent with the removal of defects, which allows relaxation of the carbon atoms 
in the film. (I.e. densification). Thus, by treatment with $\mathrm{NaBH}_{4}$, removal of defect species causes $\mathrm{C}$-atoms in the graphene film to relax and move close together, while forming a more perfect graphene layer, thus enhancing attenuation of the underlying substrate peaks. The removal of oxygen defect states was also consistent with the literature reports for graphene oxides, where the $\mathrm{H}^{+}$ion produced by dissociation of $\mathrm{NaBH}_{4}$ reacts with oxygen and epoxide defects to from $\mathrm{H}_{2} \mathrm{O}$. Even though the reduction effect was significant, sometimes treatment with high concentration of $\mathrm{NaBH}_{4}$ causes the film to be fragile due to $\mathrm{H}_{2}$ bubbles bursting under the film.

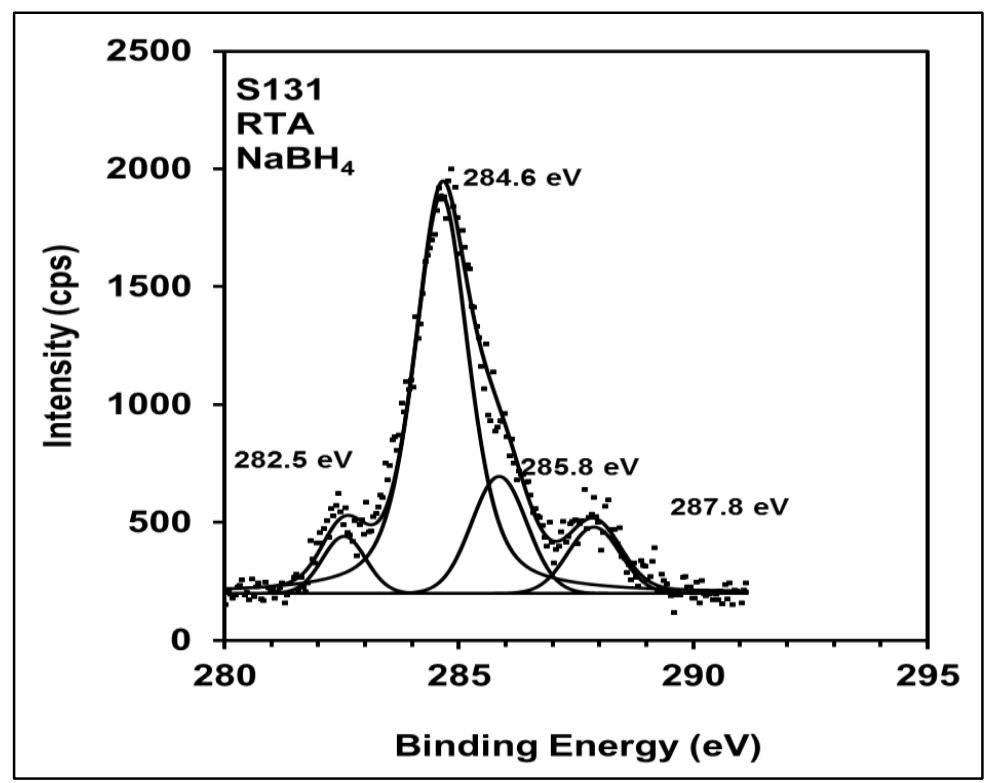

Figure 4.18: C1s XPS spectra showings structural characteristics of the graphene film after 12 hour $\mathrm{NaBH}_{4}$ reduction at room temperature.

Pei et al. and Moon et al. reported that strong reducing agents like hydroiodic acid can reduce graphene oxide without affecting the graphene film $[105,106]$. Two independent investigations using hydroiodic acid showed an increase in the $\mathrm{C} / \mathrm{O}$ ratio as well as an increase in conductivity. Here it is believed that hydrogen ions react with hydroxyl and epoxide groups to produce water, thereby eliminating oxygen from the surface. Their discussion of the role of iodine ions is not clear except to say that iodine ions become attached are readily desorbed because of the low C-I bond strength.

In our study a more readily available acid of same family, hydrochloric acid $(\mathrm{HCl})$ was selected to investigate its effect on oxygenated defects. Figure 4.19 shows the effects on graphene reduced by $\mathrm{HCl}$ for an exposure of one hour. Here, it can be seen that similar effects to that of $\mathrm{NaBH}_{4}$ were achieved at a much lower exposure time. In addition, there is a down shifting in the binding energies of the graphene and the defect states observed after exposure. As the C-atoms move closer to each other because of changes in bond length and relaxation, there is slightly greater electron orbital overlap. This increases the 
covalently shared charge on each atom and in turn increases the shielding of the core level electrons (i.e., the $\mathrm{C} 1 \mathrm{~s}$ levels). As a result, the effective binding energy of the $\mathrm{C} 1 \mathrm{~s}$ levels decreases and causes shifting in the peak location. Thus, the reduction in the defect concentration, accompanied with change in the relative distribution of defect species, can give rise to new defect species e.g. the new peak seen at 285.1 $\mathrm{eV}$. The reduction of the defect peaks indicates that their concentration on the surface has decreased from $36 \%$ to $26 \%$, as measured by high resolution XPS.

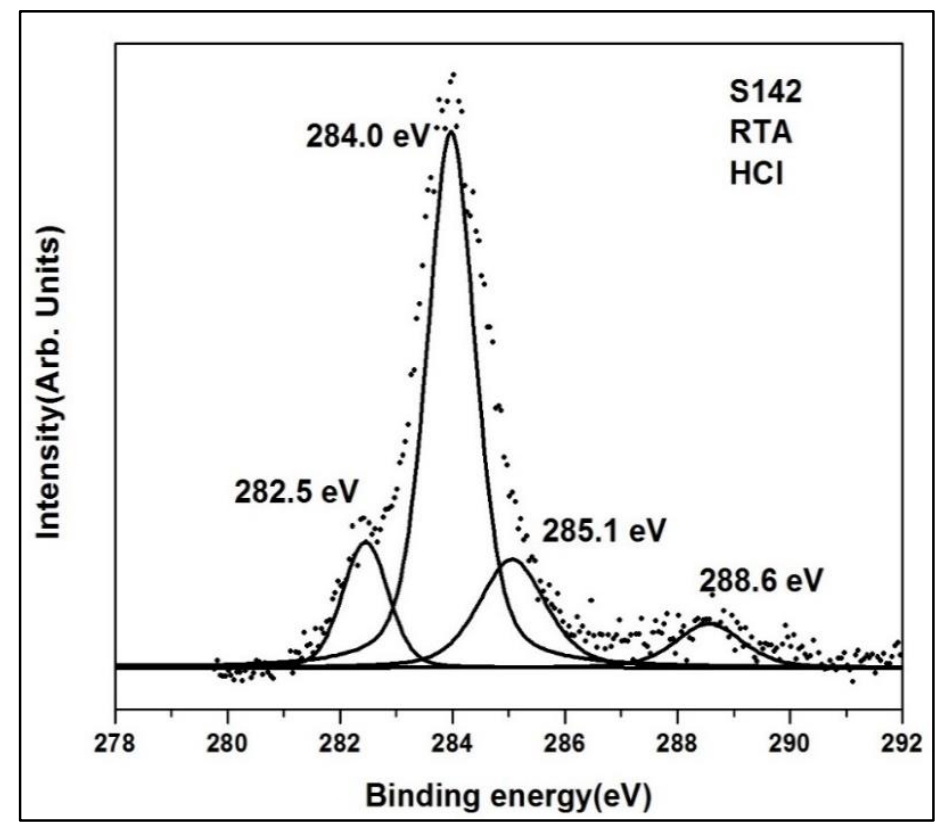

Figure 4.19: C1s XPS spectra showings structural characteristics of the graphene film after $1 \mathrm{hr} \mathrm{HCl}$ reduction at room temperature.

As the $\mathrm{HCl}$ was able to produce almost the same amount of defect reduction as $\mathrm{NaBH}_{4}$ in much less time, the effect of $\mathrm{HCl}$ was studied further to understand the capability of $\mathrm{HCl}$ to reduce defects even further. There were no chlorine peaks observed after 1 hour of treatment, suggesting that the residual atoms are present in the solution and not on the graphene film. The sample after 12 hours does show the presence of a low intensity chlorine peak, which implies that treatment for 12 hours results in an interaction of the graphene film with the residual chlorine atoms. This observation is in good agreement with similar reduction studies done on graphene oxide samples by Pei [105]. But, as there is no significant change in the electrical conductivity after 12 hours, the reduction with $\mathrm{HCl}$ can be considered stable enough for practical applications.

Since methanol, acetone and acetic acid are the most common protic and aprotic solvents for cleaning and removing residual oxide during synthesis, their effects on the graphene quality were also 
studied. With the exception of methanol, there was little if any change with these solvents and the XPS peaks almost unchanged after 12 hours. Figure 4.20 shows the effects of a 12 -hour exposure to methanol. Here, it may be seen that the SiC peak as well as the defect peaks are reduced significantly as compared to graphene peak, showing a reduction effect with methanol similar to that of $\mathrm{HCl}$. The reduction of the defect peaks by XPS indicates that their concentration on the surface has decreased from $36 \%$ to $14 \%$, less than that of the previous two reducing agents.

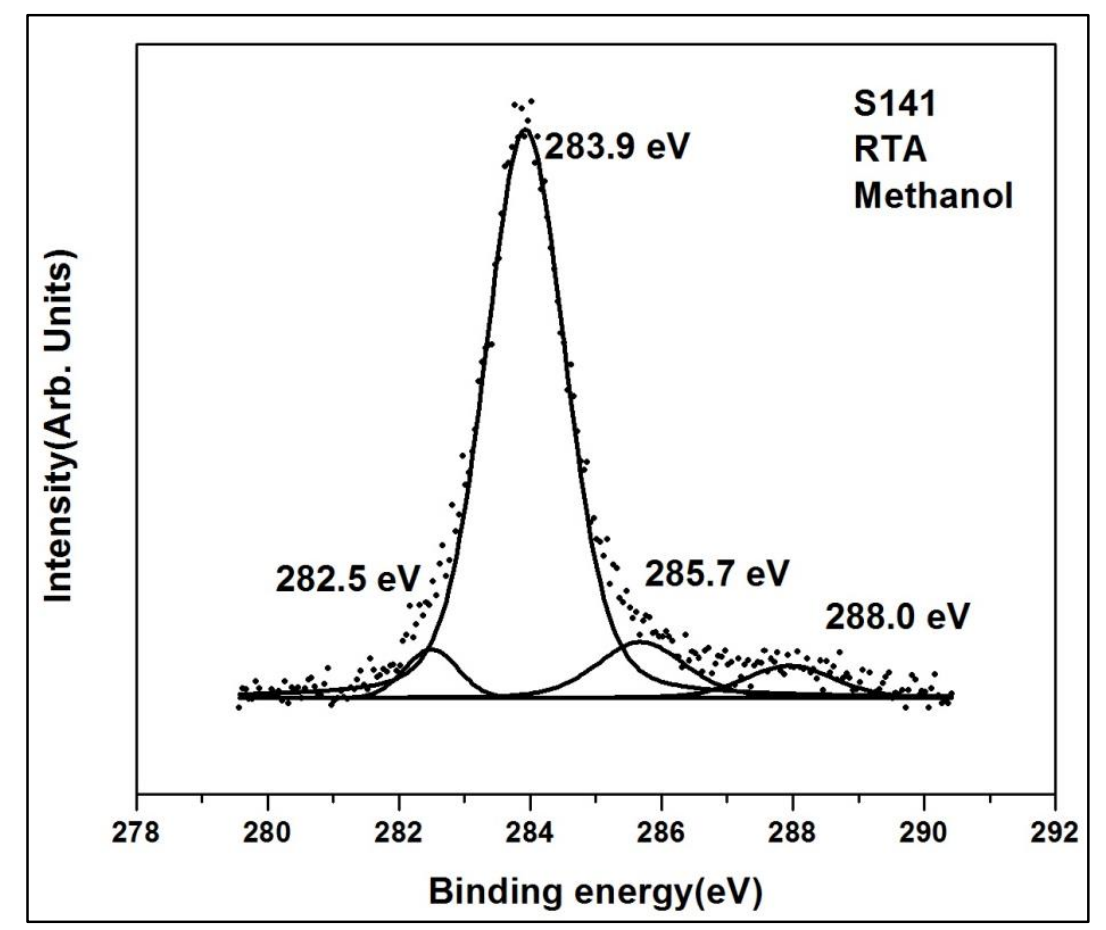

Figure 4.20: C1s XPS spectra showings structural characteristics of the graphene film after $12 \mathrm{hr}$ methanol reduction at room temperature.

\subsection{Nanoparticle Nucleation}

A key hypothesis in the present studies is that nanoparticles attached to the graphene will modify the gas adsorption and electrical characteristics and provide a basis for sensor selectivity. This section describes the results of the nucleation of $\mathrm{Ag}, \mathrm{Au}, \mathrm{Pt}$, and Ir nanoparticles on the graphene surface.

\subsubsection{General Observations of Nanoparticle Nucleation on Graphene}

Nanoparticles (NPs) were synthesized by a single-step method. In this process, the graphene was immersed in a dilute solutions of $\mathrm{NaBH}_{4}$ in $\mathrm{H}_{2} \mathrm{O}$ (2.71 and $27.1 \mathrm{mM}$ ). A dilute solution of metal salt in water ( $5 \mathrm{mM}$ to $40 \mathrm{mM}$ ) was then added dropwise to produce a nanoparticle (NP) colloid. This was generally evidenced by a dramatic change in color of the solution and has been characterized more quantitatively 
by UV-Vis spectroscopy in a number of studies [107]. The film was allowed to incubate in this solution at room temperature for several hours ( 3 hour to 48 hour) and then removed.

Figure 4.21 shows an SEM image of the surface after a 12 hour incubation period for Ag nucleation on graphene. The solution concentrations were $27.1 \mathrm{mM} \mathrm{NaBH}_{4}$ and $10 \mathrm{mM} \mathrm{AgNO}_{3}$. It can be seen that there are a large number of particles and clusters of particles on the sample. The majority of these are much larger than the desired nanoparticles. Closer examination revealed that most of these particles had a spheroidal shape and appeared to be only weakly attached. This was simply the result of sedimentation onto the surface of the film. That is, these particles were homogeneously nucleated in the solution and not on the surface of graphene.

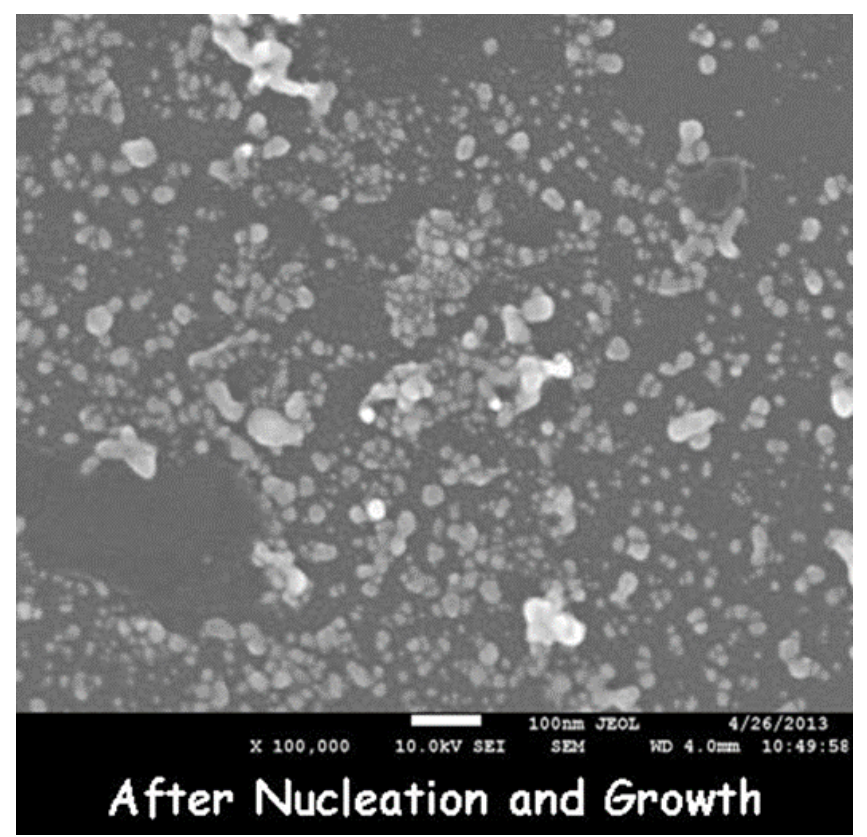

Figure 4.21: SEM images of graphene surface after 12 hour incubation period for silver nucleation.

Figure 4.22 shows the same surface after 5 minutes of sonication in acetone. Here it can be seen that only a few of the larger particles of 30 to $40 \mathrm{~nm}$ (squares) and a large number of smaller particles of size 1 to $5 \mathrm{~nm}$ remain (e.g. several are circled but most are not). XPS was used to confirm the presence of $\mathrm{Ag}$ on this surface at nominally at a concentration of $1.3 \%$. It is worth noting that after some experimentation, the same level of particle removal could be achieved with a simple jet of deionized (DI) water. Thus, in subsequent studies sonication was replaced by the simpler and faster DI jet rinse. To further characterize the nanoparticles which remained attached to the surface, AFM analyses were performed. 


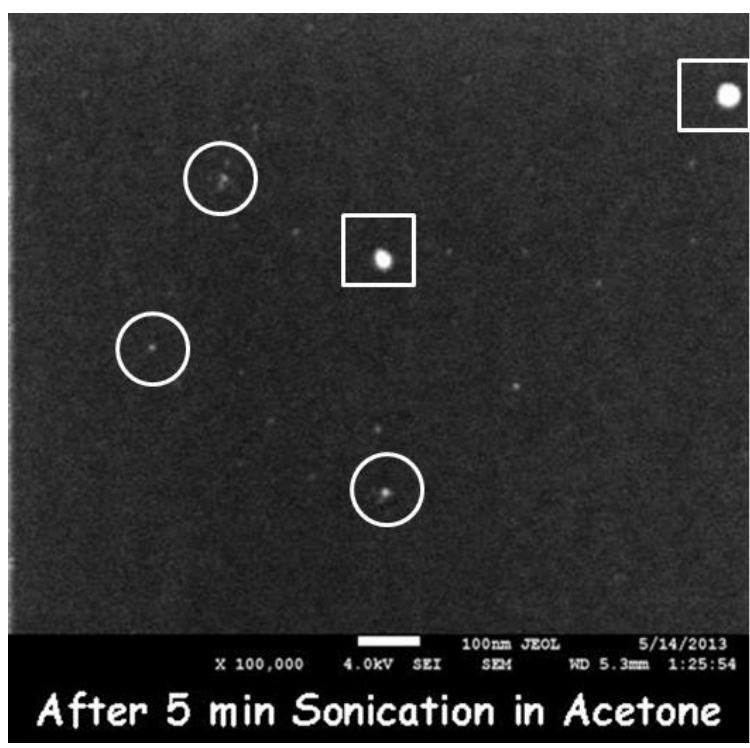

Figure 4.22: SEM images of graphene surface after 5 min sonication in acetone for silver nucleation after 12 hour incubation period.

Figure 4.23 (a) shows a $2 \mu \mathrm{m} \times 2 \mu \mathrm{m}$ region of a similarly prepared surface with Au NPs. Here a relatively uniform distribution of NPs can be seen with many separated particles and some clusters. The overall RMS of the surface is $6.0 \mathrm{~nm}$. Figure 4.23 (b) is the $500 \mathrm{~nm} \times 500 \mathrm{~nm}$ region identified by the square in Figure 4.23 (a). Here again, clusters and a large number of individual NPs can be observed. The overall RMS for this image is $5.8 \mathrm{~nm}$. The adjacent line profile shows the NPs range in height from 0.5 $\mathrm{nm}$ to $6 \mathrm{~nm}$ and have diameters of up to $50 \mathrm{~nm}$. Figure 4.23 (c) is the $100 \mathrm{~nm} \times 100 \mathrm{~nm}$ region identified in Figure 4.23 (b).At this scale only two NPs can be observed on the otherwise smooth graphene surface. Consequently the overall RMS for this image has dropped to $\sim 0.4 \mathrm{~nm}$ characteristic of the graphene surface. The adjacent line profiles show the low level hillocks characteristic of graphene (lower profile) and a single particle (upper). In this case the particle is on the order of $2 \mathrm{~nm}$ in height and $20 \mathrm{~nm}$ in diameter.

This behavior was typical of virtually all NPs and all NPs systems (i.e., Ag, Au, Pt, and Ir) were examined. With the exception of some large particles remaining after either the sonication or water jet rinse, these results suggest that the smaller particles heterogeneously nucleate on the graphene surface and grow by a Volmer-Webber growth mechanism as illustrated in Figure 4.23 (d).In this mechanism, particle growth is three-dimensional since metal-metal interactions are stronger than metal carbon interactions.

Before describing the individual nanoparticle systems in detail, it is worth noting here that the particle nucleation process can be done either before or after the deposition of metal contacts. It was found, however, that wire bonds formed on Ti/Au electrical contacts deposited over the nanoparticles 
were not as robust as those formed on contacts directly deposited on graphene. It was also found that sonication degraded the metal contacts. Although no systematic studies were done to explore the damage mechanism, it most likely this involves micro-cavitation. The point here is that the optimum device fabrication sequence compatible with these constraints involves graphene synthesis followed by contact deposition, followed by nanoparticle nucleation with water jet removal of the homogeneously nucleated particles. As a further constraint, it was observed that high concentrations of $\mathrm{NaBH}_{4}$ (i.e., $27.1 \mathrm{mM}$ ) degraded the integrity of the electrical contacts. Thus, for device fabrication (as opposed to simply studying the nucleation process), the $\mathrm{NaBH}_{4}$ concentration was kept a factor of 10 lower (i.e., $2.71 \mathrm{mM}$ ). 

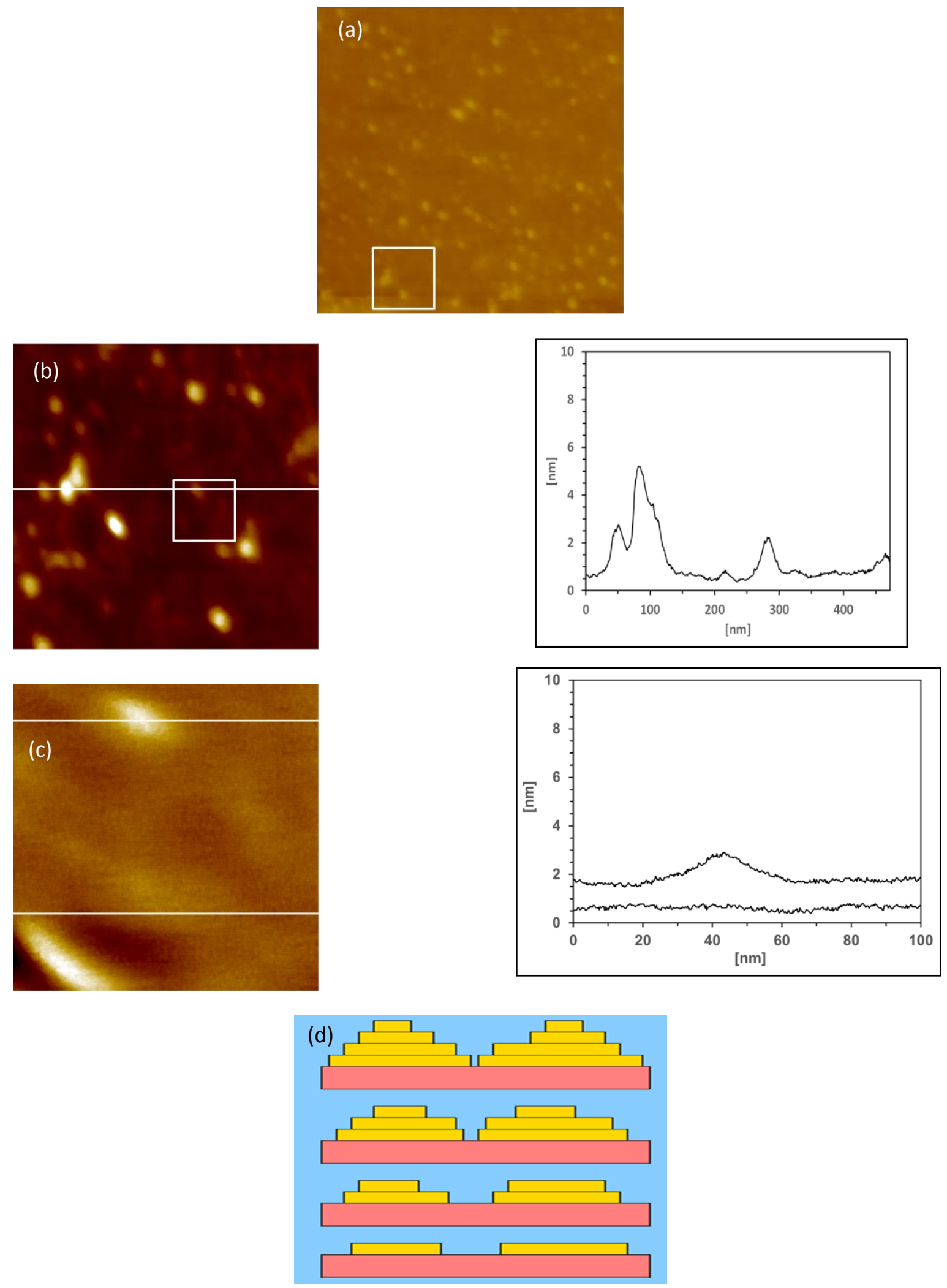

Figure 4.23: (a) $2 \mu m \times 2 \mu m$ AFM image of Au NPs on graphene, b) $500 \mathrm{~nm} \times 500 \mathrm{~nm}$ image of square region in (a) with corresponding lines scan, c) $100 \mathrm{~nm} \times 100 \mathrm{~nm}$ image of square region in (b) and corresponding line scans, and d) schematic of Volmer-Webber growth mechanism 


\subsubsection{Silver Nanoparticle Nucleation}

The basic reaction for formation of Ag NPs is:

$$
\mathrm{AgNO}_{3}+\mathrm{NaBH}_{4} \rightarrow \mathrm{Ag}+1 / 2 \mathrm{H}_{2}+1 / 2 \mathrm{~B}_{2} \mathrm{H}_{6}+\mathrm{NaNO}_{3}
$$

Figure 4.24 shows the change in color of the solution from the colorless $\mathrm{NaBH}_{4}$ solution in water to a pale yellow upon addition of $\mathrm{AgNO}_{3}$, confirming the formation of silver colloid. In the detailed studies of the nucleation process, the concentration of $\mathrm{NaBH}_{4}$ was limited to $2.71 \mathrm{mM}$ because of its effect on the metal contacts at higher concentrations of $\mathrm{NaBH}_{4} . \mathrm{AgNO}_{3}$ concentrations of $5 \mathrm{mM}$ and $10 \mathrm{mM}$ both produced bright yellow stable colloids of $\mathrm{Ag} \mathrm{NPs}$, but the $5 \mathrm{mM} \mathrm{AgNO}_{3}$ solution, in this case, produced very few heterogeneously nucleated particles even after 12 hours. Consequently, incubation time studies were performed only for the $10 \mathrm{mM} \mathrm{AgNO}_{3}$ solution. Figures 4.25, 4.26, and 4.27 show results for 3 hour, 6 hour, and 12 hour incubation times, respectively. Each figure includes a $5 \mu \mathrm{m} \times 5 \mu \mathrm{m}$ AFM image along with data on the particle size distribution. Figure 4.28 shows the corresponding SEM images. Table 4.1 shows summarizes the AFM data, while Table 4.2 summarizes the XPS data for these nucleation conditions. It is immediately clear from the AFM data that the areal density of particles increases with incubation time as does the surface coverage. However, the mean particle diameter and height remain essentially unchanged at $\sim 35 \mathrm{~nm}$ diameter and $\sim 2 \mathrm{~nm}$ height. This is also reflected in the XPS data which shows that the atomic percent of $\mathrm{Ag}$ increases with incubation time. Finally, the SEM images in Figure 4.28 , although less quantitative, are consistent with these observations.

In summary, the areal density, coverage, and size distribution at 6 hour and 12 hour are essentially in the desired range for sensor development. If sensor development studies require greater coverage, however, higher concentrations of $\mathrm{AgNO}_{3}$ may be considered.

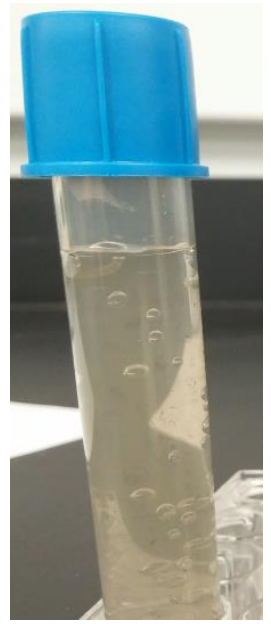

Figure 4.24: Change in color from colorless to pale yellow after 12 hours, confirming formation of silver colloid. 


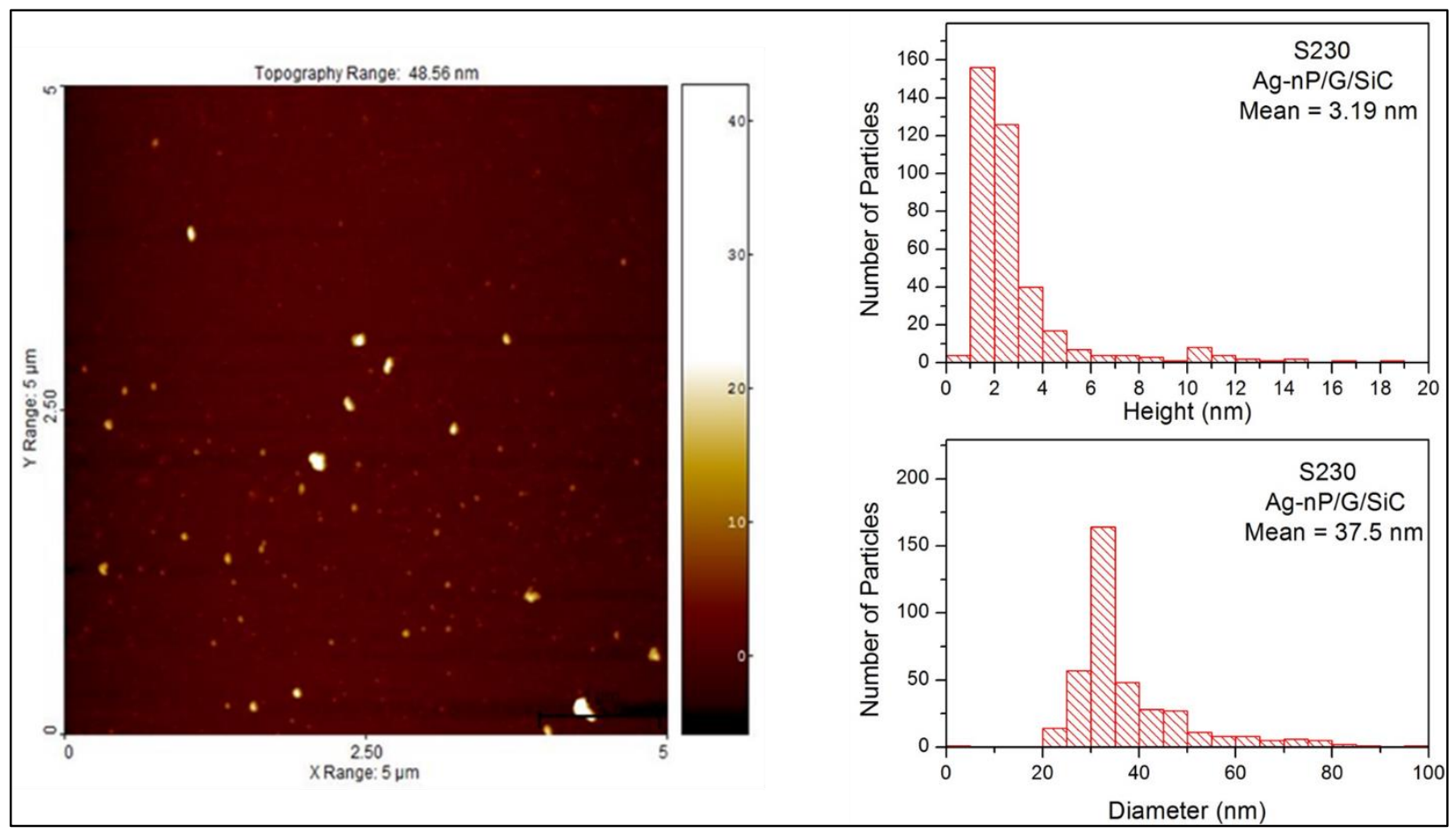

Figure 4.25: AFM image of the AgNPs, obtained with $2.71 \mathrm{mM} \mathrm{NaBH}_{4}$ and $10 \mathrm{mM} \mathrm{AgNO}_{3}$, after 3 hours of incubation time, with the histogram showing AgNPs size and height distribution.

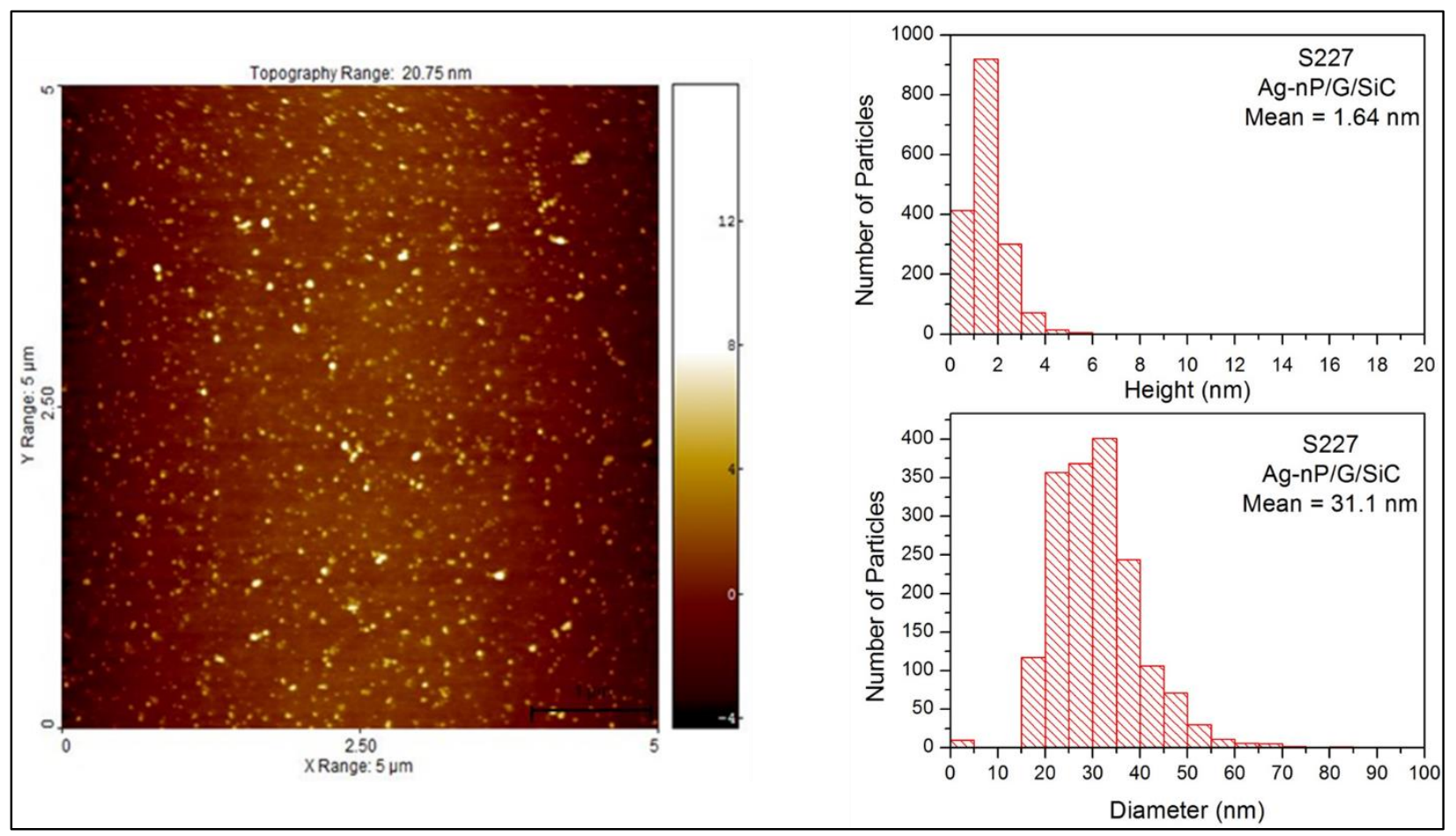

Figure 4.26: AFM images of the AgNPs, obtained with $2.71 \mathrm{mM} \mathrm{NaBH}_{4}$ and $10 \mathrm{mM} \mathrm{AgNO}_{3}$, after 6 hours of incubation time, with the histogram showing AgNPs size and height distribution. 


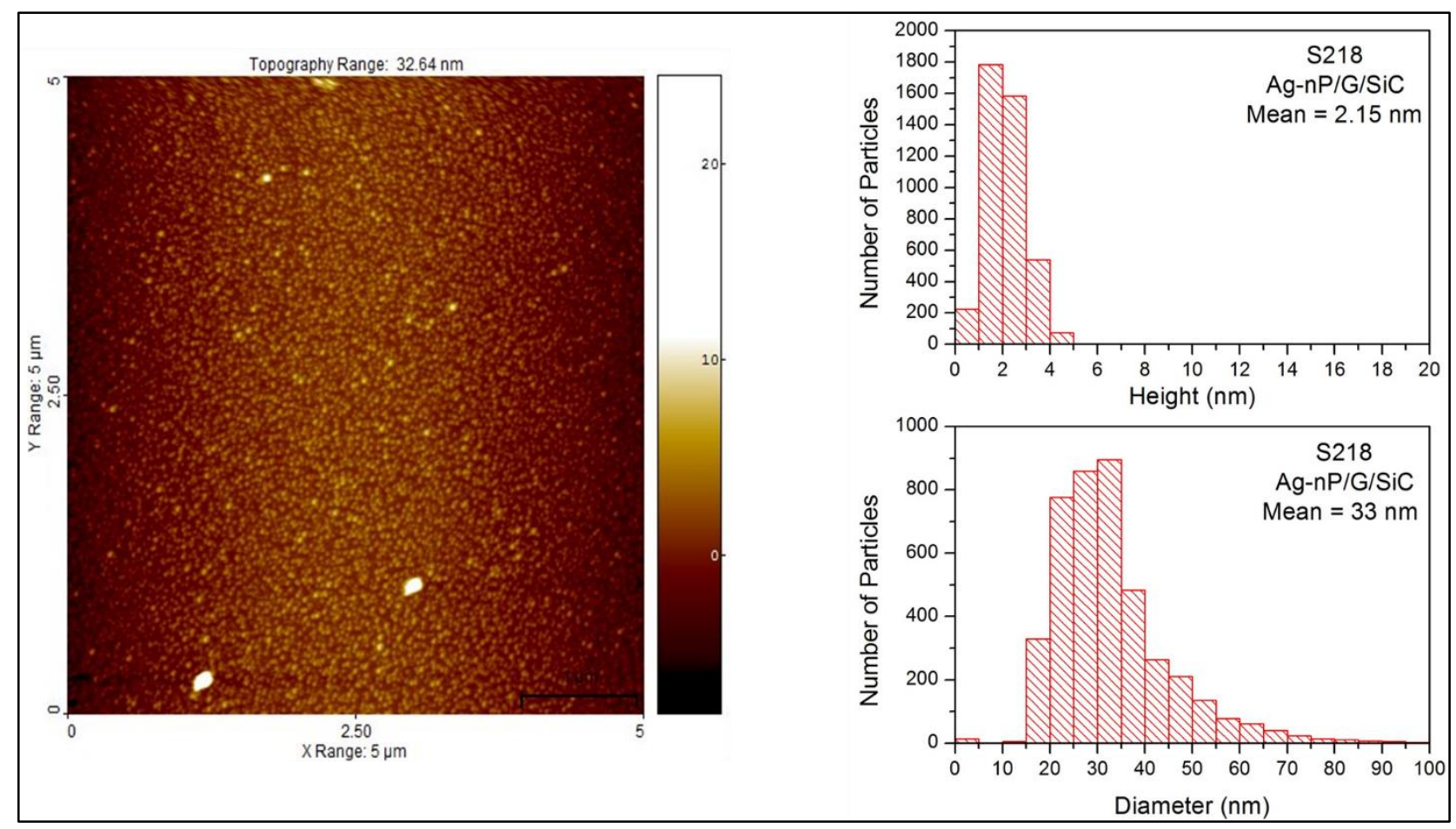

Figure 4.27: AFM images of the AgNPs, obtained with $2.71 \mathrm{mM} \mathrm{NaBH}_{4}$ and $10 \mathrm{mM} \mathrm{AgNO}_{3}$, after 12 hour of incubation time, with the histogram showing AgNPs size and height distribution.
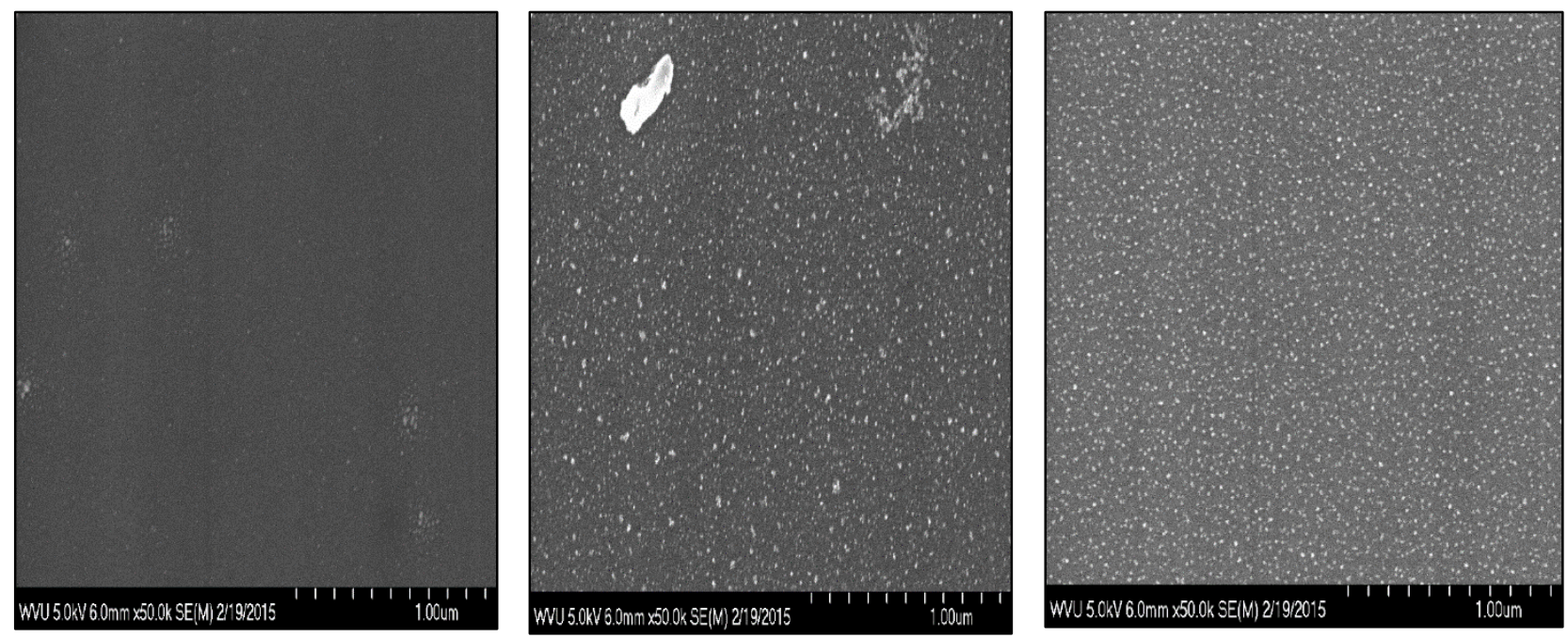

Figure 4.28: SEM images of the AgNPs, obtained with $2.71 \mathrm{mM} \mathrm{NaBH}_{4}$ and $10 \mathrm{mM} \mathrm{AgNO}_{3}$, after 3, 6 and 12 hours of incubation time. 
Table 4.1: AFM Analysis of Silver Nanoparticles for $2.71 \mathrm{mM} \mathrm{NaBH}_{4}$ and $10 \mathrm{mM} \mathrm{AgNO}_{3}$.

\begin{tabular}{|c|c|c|c|c|}
\hline Time & $\begin{array}{c}\text { Number of } \\
\text { particles }\end{array}$ & Coverage $(\%)$ & $\begin{array}{c}\text { Mean } \\
\text { diameter(nm) }\end{array}$ & Mean height(nm) \\
\hline 3 hour & 386 & 1.88 & 37.25 & 3.19 \\
\hline 6 hour & 1729 & 5.87 & 31.3 & 1.54 \\
\hline 12 hour & 4125 & 16.29 & 33 & 2.15 \\
\hline
\end{tabular}

Table 4.2: XPS analysis for silver nanoparticles for $2.71 \mathrm{mM} \mathrm{NaBH}_{4}$ and $10 \mathrm{mM} \mathrm{AgNO}_{3}$

\begin{tabular}{|c|c|c|c|c|}
\hline Time & C1s (\%) & O1s (\%) & Si2s (\%) & Ag3d (\%) \\
\hline 3 hour & 46.06 & 23.67 & 29.53 & 0.73 \\
\hline 6 hour & 46.79 & 30.23 & 22.09 & 0.89 \\
\hline 12 hour & 44.14 & 26.46 & 28.4 & 0.99 \\
\hline
\end{tabular}

\subsubsection{Gold Nanoparticle Nucleation}

The basic reaction for the formation of Au NPs is:

$$
2 \mathrm{HAuCl}_{4}+3 \mathrm{NaBH}_{4} \rightarrow 2 \mathrm{Au}+3 \mathrm{BH}_{4}+5 \mathrm{HCl}+3 \mathrm{NaCl}
$$

Figure 4.29 shows the change in color of the solution from colorless $\mathrm{NaBH}_{4}$ solution in water to pale purple upon addition of $\mathrm{HAuCl}_{4}$, confirming the formation of a gold colloid. In the detailed studies of the nucleation process, the concentration of $\mathrm{NaBH}_{4}$ was limited to $2.71 \mathrm{mM}$ because of its effect on the metal contacts at high concentrations. $\mathrm{HAuCl}_{4}$ concentrations of $4.8 \mathrm{mM}$ and $9.6 \mathrm{mM}$ both produced pale purple stable colloids of Au NPs, but the $4.8 \mathrm{mM} \mathrm{HAuCl}_{4}$ solution, in this case, produced very few heterogeneously nucleated particles even after 12 hours. Consequently, incubation time studies were performed only for the $9.6 \mathrm{mM} \mathrm{HAuCl}_{4}$ solution. Figure 4.30 shows results for 3 hour, 6 hour, and 12 hour incubation times. Each figure includes a $5 \mu \mathrm{m} \times 5 \mu \mathrm{m}$ AFM image along with particle size distribution data. Table 4.3 shows summarizes the AFM data, while Table 4.4 summarizes the XPS data for these nucleation conditions. It is immediately clear from the AFM data that the areal density of particles increases with incubation time as does the surface coverage. However, the mean particle diameter and height remain essentially unchanged at $\sim 55 \mathrm{~nm}$ diameter and $\sim 2 \mathrm{~nm}$ height. This is also reflected in the XPS data which show that the atomic percent of $\mathrm{Au}$ increases incubation time. 
In summary, the areal density, coverage, and size distribution at 6 hour and 12 hour are essentially in the desired range for sensor development. However, it was seen that the number of particles formed using gold nucleation was much lower than that of silver. This might be due to slower reaction rate for gold nanoparticle nucleation. Thus, in order to get greater coverage of nanoparticle, metal salt concentration was varied from $9.6 \mathrm{mM}$ to $19.2 \mathrm{mM}$ (2 times the original concentration) and $38.4 \mathrm{mM}$ (4 times the original concentration) and keeping the incubation time of 12 hour.

Figure 4.31 shows the results for $9.6 \mathrm{mM}, 19.2 \mathrm{mM}$ and $38.4 \mathrm{mM}$ concentrations of $\mathrm{HAuCl}_{4}$, respectively. Each figure includes a $5 \mu \mathrm{m} \times 5 \mu \mathrm{m}$ AFM image along with data on the particle size distribution. Table 4.5 summarizes the AFM data, while Table 4.6 summarizes the XPS data for these nucleation conditions. It is immediately clear from the AFM data that the areal density of particles increases with an increase in salt concentration as does the surface coverage. This might reflect on increase in particle flux with higher concentrations of salt. However, the mean particle diameter and height remain essentially unchanged at $\sim 55 \mathrm{~nm}$ diameter and $\sim 1-2 \mathrm{~nm}$ height, showing almost no change in the geometry of the particles with concentration. This is also reflected in the XPS data which show that the atomic percent of Au increases with incubation time.

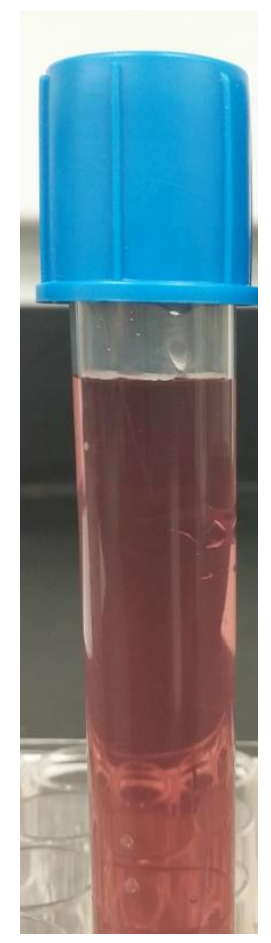

Figure 4.29: Change in color from colorless to pale purple, confirming formation of gold colloid. 

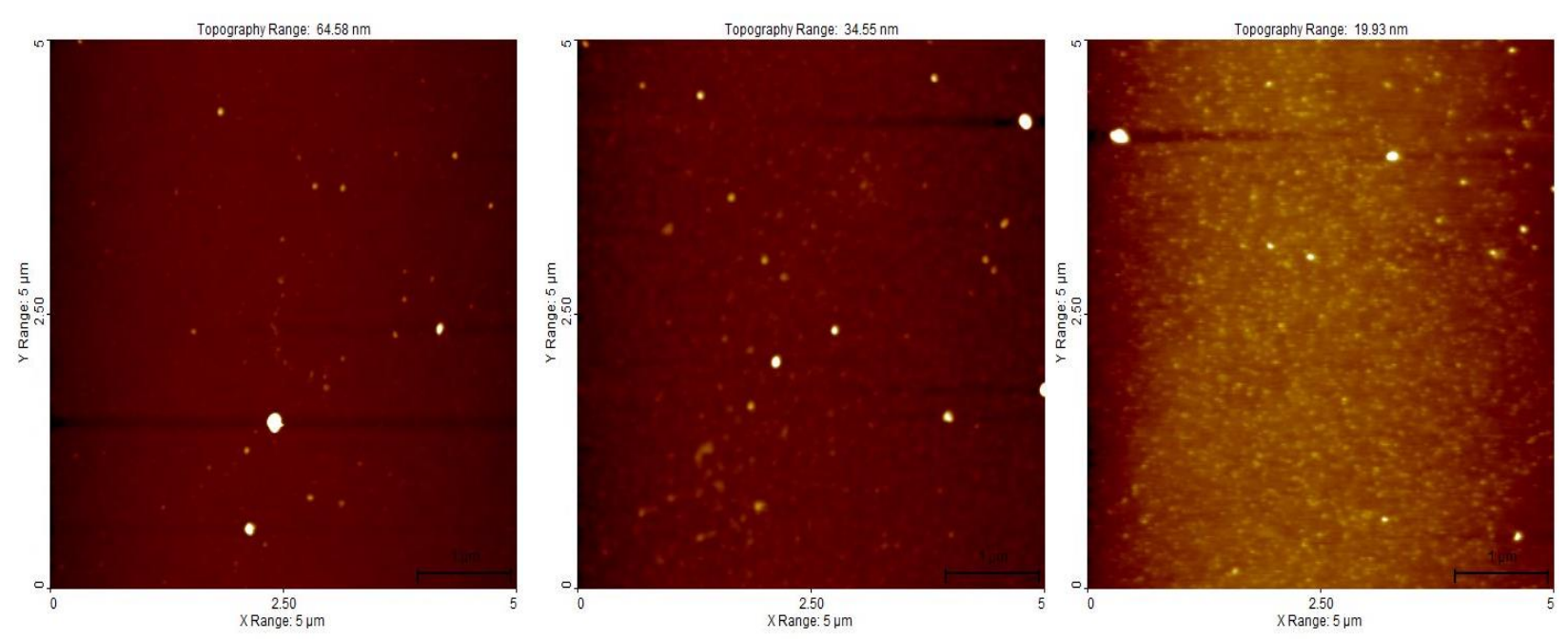

Figure 4.30: AFM images of the AuNPs, obtained with $2.71 \mathrm{mM} \mathrm{NaBH}_{4}$ and $9.6 \mathrm{mM} \mathrm{HAuCl}_{4}$, after $3 \mathrm{hr}, 6$ hr and 12 hr of incubation time.

Table 4.3: AFM Analysis of Gold Nanoparticles for $2.71 \mathrm{mM} \mathrm{NaBH}_{4}$ and $9.6 \mathrm{mM} \mathrm{HAuCl}_{4}$.

\begin{tabular}{|c|c|c|c|c|}
\hline Time & $\begin{array}{c}\text { Number of } \\
\text { particles }\end{array}$ & Coverage (\%) & $\begin{array}{c}\text { Mean } \\
\text { diameter(nm) }\end{array}$ & Mean height(nm) \\
\hline 3 hour & 14 & 0.16 & 57 & 6.64 \\
\hline 6 hour & 61 & 1.01 & 70.5 & 2.55 \\
\hline 12 hour & 207 & 1.98 & 52.5 & 1.12 \\
\hline
\end{tabular}

Table 4.4: XPS analysis for silver nanoparticles for $2.71 \mathrm{mM} \mathrm{NaBH}_{4}$ and $9.6 \mathrm{mM} \mathrm{HAuCl}_{4}$.

\begin{tabular}{|c|c|c|c|c|}
\hline Time & C1s (\%) & O1s (\%) & Si2s (\%) & Au4f (\%) \\
\hline 3hour & 67.04 & 19.22 & 13.47 & 0.27 \\
\hline 6 hour & 45.19 & 25.62 & 28.89 & 0.31 \\
\hline 12 hour & 58.08 & 23.95 & 17.06 & 0.92 \\
\hline
\end{tabular}



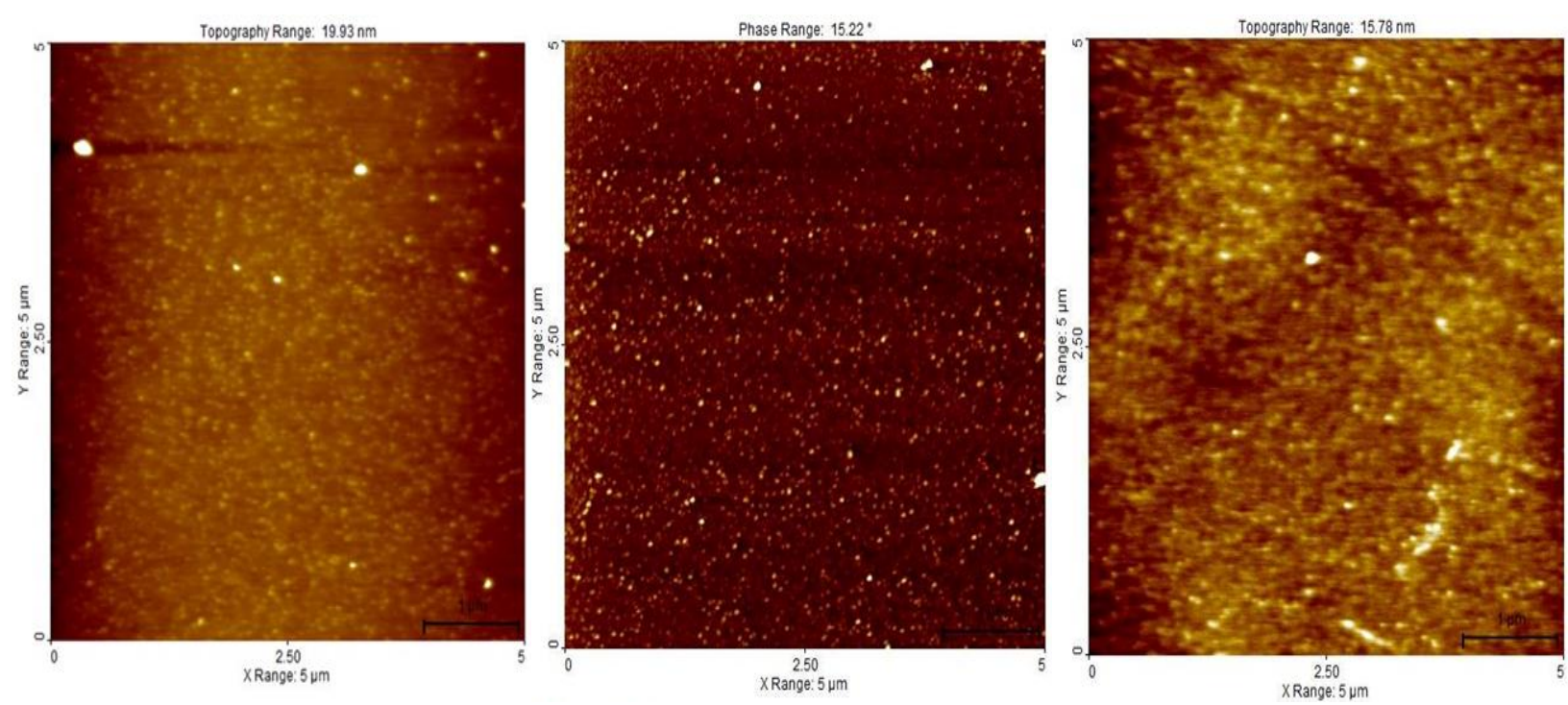

Figure 4.31: AFM images of the AuNPs, obtained with $2.71 \mathrm{mM} \mathrm{NaBH}_{4}$ and 12 hour incubation period for 9.6 $\mathrm{mM}, 19.2 \mathrm{mM}$ and $38.4 \mathrm{mM}$ concentrations of $\mathrm{HAuCl}_{4}$ respectively.

Table 4.5: AFM Analysis for Gold Nanoparticles for $2.71 \mathrm{mM} \mathrm{NaBH}_{4}$ and 12 hour incubation time for 9.6 $\mathrm{mM}, 19.2 \mathrm{mM}$ and $38.4 \mathrm{mM}$ concentrations of $\mathrm{HAuCl}_{4}$.

\begin{tabular}{|c|c|c|c|c|}
\hline Time & $\begin{array}{c}\text { Number of } \\
\text { particles }\end{array}$ & $\begin{array}{c}\text { Coverage } \\
(\%)\end{array}$ & $\begin{array}{c}\text { Mean } \\
\text { diameter(nm) }\end{array}$ & Mean height(nm) \\
\hline 12 hour $(9.6 \mathrm{mM})$ & 207 & 1.98 & 52.5 & 1.12 \\
\hline 12 hour $(19.2 \mathrm{mM})$ & 718 & 8.80 & 54.8 & 0.49 \\
\hline 12 hour $(38.4 \mathrm{mM})$ & 1149 & 12.86 & 52.9 & 1.04 \\
\hline
\end{tabular}

Table 4.6: XPS Analysis for Gold Nanoparticles for $2.71 \mathrm{mM} \mathrm{NaBH}$ and 12 hour incubation time for 9.6 $\mathrm{mM}, 19.2 \mathrm{mM}$ and $38.4 \mathrm{mM}$ concentrations of $\mathrm{HAuCl}_{4}$.

\begin{tabular}{|c|c|c|c|c|}
\hline Time & C1s (\%) & O1s (\%) & Si2s (\%) & Au4f (\%) \\
\hline 12 hour(9.6 mM) & 58.08 & 23.95 & 17.06 & 0.92 \\
\hline 12 hour(19.2 mM) & 64.45 & 18.36 & 14.36 & 2.82 \\
\hline 12 hour(38.4 mM) & 60.03 & 17.91 & 17.38 & 4.68 \\
\hline
\end{tabular}




\subsubsection{Platinum Nanoparticle Nucleation}

The basic reaction for the formation of Pt NPs is?

$$
2 \mathrm{H}_{2} \mathrm{PtCl}_{6}+3 \mathrm{NaBH}_{4} \rightarrow 2 \mathrm{Pt}+3 \mathrm{NaCl}+3 \mathrm{BCl}_{3}+8 \mathrm{H}_{2}
$$

In the detailed studies of the nucleation process, the concentration of $\mathrm{NaBH}_{4}$ was limited to 2.71 $\mathrm{mM}$ because of its effect on the metal contacts at high concentrations. $\mathrm{H}_{2} \mathrm{PtCl}_{6}$ concentrations of 1.25 $\mathrm{mM}, 2.5 \mathrm{mM}$ and $5 \mathrm{mM}$ produced brown and stable colloids of Pt NPs, but the 1.25 and $2.5 \mathrm{mM} \mathrm{H}_{2} \mathrm{PtCl}_{6}$ solution, in this case, produced very few heterogeneously nucleated particles even after 12 hours. Consequently, incubation time studies were performed only for the $5 \mathrm{mM} \mathrm{H}_{2} \mathrm{PtCl}_{6}$ solution. Figure 4.32 shows results for 24 hour, 36 hour, and 48 hour incubation times. Each figure includes a $5 \mu \mathrm{m} \times 5 \mu \mathrm{m}$ AFM image along with data on the particle size distribution data. Table 4.7 summarizes the AFM data, while Table 4.8 summarizes the XPS data for these nucleation conditions. It is immediately clear from the AFM data that the areal density of particles increases with incubation time as does the surface coverage. However, the mean particle diameter and height remain essentially unchanged at $\sim 60 \mathrm{~nm}$ diameter and $\sim 1.5 \mathrm{~nm}$ height. This is also reflected in the XPS data which show that the atomic percent of Pt increases with incubation time.

In summary, the areal density, coverage, and size distribution at 36 hour and 48 hour are essentially in the desired range for sensor development. Again, it was seen that the number of particles formed using Pt nucleation was much lower than that of the silver. Thus, in order to get greater coverage of nanoparticle, the metal salt concentration was varied from $5 \mathrm{mM}$ to $10 \mathrm{mM}$ ( 2 times the original concentration) and $20 \mathrm{mM}$ (4 times the original concentration) and keeping the incubation time of 24 hour.

Figure 4.33 shows the results for the $5 \mathrm{mM}, 10 \mathrm{mM}$ and $20 \mathrm{mM}$ concentrations of $\mathrm{H}_{2} \mathrm{PtCl}_{6}$, respectively. Each figure includes a $5 \mu \mathrm{m} \times 5 \mu \mathrm{m}$ AFM image along with data on the particle size distribution. Table 4.9 summarizes the AFM data, while Table 4.10 summarizes the XPS data for these nucleation conditions. It is immediately clear that the AFM data show same behavior as that of gold. However, the mean particle diameter and height remain essentially unchanged at $\sim 50-70 \mathrm{~nm}$ diameter and 1-2 nm height, showing almost no change in geometry of particles with concentration. This is also reflected in the XPS data which show that the atomic percent of Au increases with incubation time. Figure 4.34 shows the change in color of the solution from the colorless $\mathrm{NaBH}_{4}$ solution in water to a brown color upon addition of $\mathrm{H}_{2} \mathrm{PtCl}_{6}$, confirming formation of platinum colloid. 

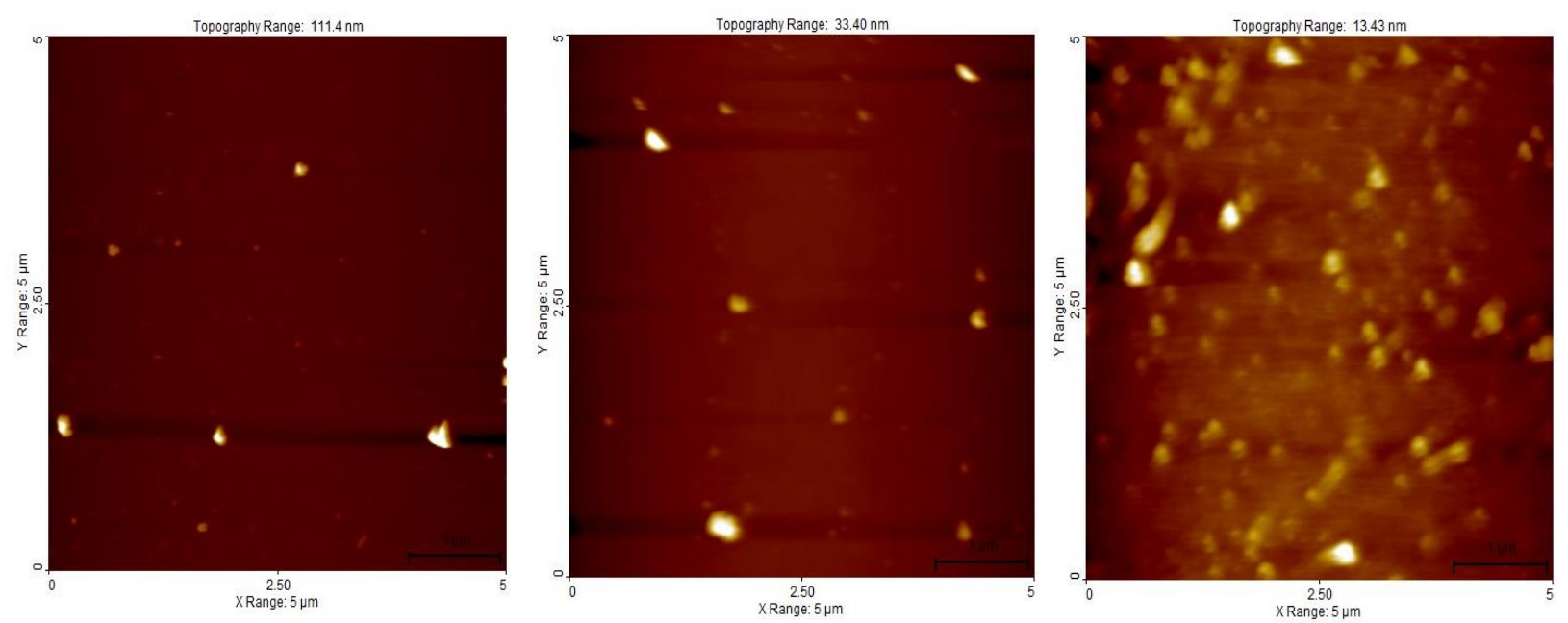

Figure 4.32: AFM images of the PtNPs, obtained with $2.71 \mathrm{mM} \mathrm{NaBH}_{4}$ and $5 \mathrm{mM} \mathrm{H}_{2} \mathrm{PtCl}_{6}$, after $24 \mathrm{hr}, 36$ hr and 48 hr of incubation time.

Table 4.7: AFM Analysis for Platinum Nanoparticles with $2.71 \mathrm{mM} \mathrm{NaBH}_{4}$ and $5 \mathrm{mM} \mathrm{H}_{2} \mathrm{PtCl}_{6}$, after 24 hour, 36 hour and 48 hour of incubation time.

\begin{tabular}{|c|c|c|c|c|}
\hline Time & $\begin{array}{c}\text { Number of } \\
\text { particles }\end{array}$ & Coverage $(\%)$ & $\begin{array}{c}\text { Mean } \\
\text { diameter(nm) }\end{array}$ & Mean height(nm) \\
\hline 24 hour & 39 & 0.65 & 68.2 & 12.81 \\
\hline 36 hour & 110 & 1.47 & 57.3 & 1.54 \\
\hline 48 hour & 278 & 10.7 & 103.4 & 1.20 \\
\hline
\end{tabular}

Table 4.8: XPS analysis with Platinum Nanoparticles $2.71 \mathrm{mM} \mathrm{NaBH}_{4}$ and $5 \mathrm{mM} \mathrm{H}_{2} \mathrm{PtCl}_{6}$, after 24 hour, 36 hour and 48 hour of incubation time.

\begin{tabular}{|c|c|c|c|c|}
\hline Time & C1s (\%) & O1s (\%) & Si2s (\%) & Pt4f (\%) \\
\hline 24 hour & 56.84 & 22.6 & 20.49 & 0.07 \\
\hline 36 hour & 52.72 & 20.91 & 26.27 & 0.11 \\
\hline 48 hour & 56.59 & 25.47 & 17.36 & 0.58 \\
\hline
\end{tabular}



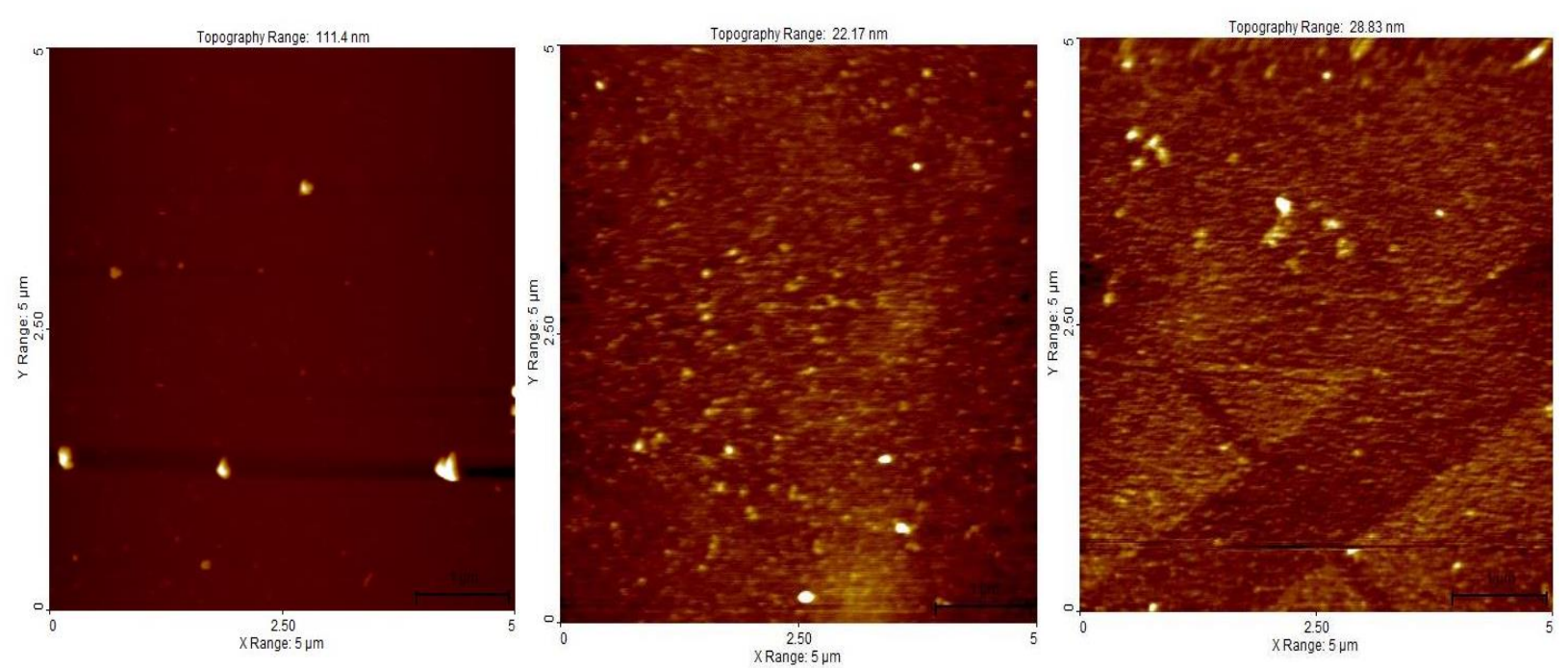

Figure 4.33: AFM images of the PtNPs, obtained with $2.71 \mathrm{mM} \mathrm{NaBH}_{4}$ and $24 \mathrm{hr}$ incubation period for 5 $\mathrm{mM}, 10 \mathrm{mM}$ and $20 \mathrm{mM}$ concentration of $\mathrm{H}_{2} \mathrm{PtCl}_{6}$ respectively.

Table 4.9: AFM Analysis for Platinum Nanoparticles with $2.71 \mathrm{mM} \mathrm{NaBH}_{4}$ and 24 hour incubation period for $5 \mathrm{mM}, 10 \mathrm{mM}$ and $20 \mathrm{mM}$ concentration of $\mathrm{H}_{2} \mathrm{PtCl}_{6}$ respectively.

\begin{tabular}{|c|c|c|c|c|}
\hline Time & $\begin{array}{c}\text { Number of } \\
\text { particles }\end{array}$ & Coverage (\%) & $\begin{array}{c}\text { Mean } \\
\text { diameter(nm) }\end{array}$ & Mean height(nm) \\
\hline 24 hour(5 mM) & 39 & 0.65 & 68.2 & 12.81 \\
\hline 24 hour(10 mM) & 516 & 4.217 & 47.3 & 1.21 \\
\hline 24 hour(20 mM) & 1160 & 12.58 & 52.9 & 1.05 \\
\hline
\end{tabular}

Table 4.10: XPS Analysis for Platinum Nanoparticles with $2.71 \mathrm{mM} \mathrm{NaBH}_{4}$ and 24 hour incubation period for $5 \mathrm{mM}, 10 \mathrm{mM}$ and $20 \mathrm{mM}$ concentration of $\mathrm{H}_{2} \mathrm{PtCl}_{6}$ respectively.

\begin{tabular}{|c|c|c|c|c|}
\hline Time & C1s (\%) & O1s (\%) & Si2s (\%) & Pt4f (\%) \\
\hline 24 hour $(5 \mathrm{mM})$ & 56.84 & 22.6 & 20.49 & 0.07 \\
\hline 24 hour $(10 \mathrm{mM})$ & 60.61 & 29.66 & 9.17 & 0.56 \\
\hline 24 hour $(20 \mathrm{mM})$ & 62.60 & 17.36 & 16.25 & 3.79 \\
\hline
\end{tabular}




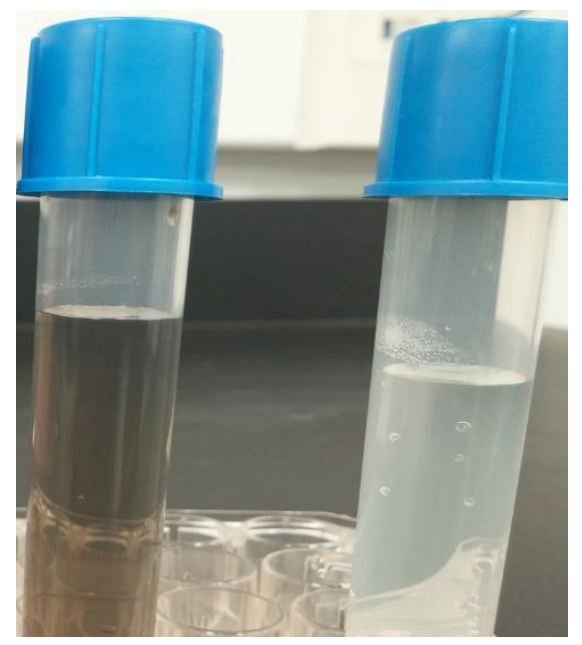

Figure 4.34: Change in color from colorless to brown(left) and from drak brown to colorless(right), confirming formation of platinum and Iridium colloid, respectively.

\subsubsection{Iridium Nanoparticle Nucleation}

The basic reaction for the formation of Ir NPs is:

$$
2 \mathrm{H}_{2} \mathrm{IrCl} 6+3 \mathrm{NaBH}_{4} \rightarrow 2 \mathrm{Ir}+3 \mathrm{NaCl}+3 \mathrm{BCl}_{3}+8 \mathrm{H}_{2}
$$

Figure 4.34 shows the change in color of the solution from colored brown solution of $\mathrm{H}_{2} \mid \mathrm{rCl}_{6}$ in water to colorless after addition into $\mathrm{NaBH}_{4}$ solution, confirming formation of Iridium colloid. In the detailed studies of the nucleation process, the concentration of $\mathrm{NaBH}_{4}$ was limited to $2.71 \mathrm{mM}$ because of its effect on the metal contacts at high concentrations. $\mathrm{H}_{2} \mathrm{IrCl}_{6}$ concentrations of $1.25 \mathrm{mM}, 2.5 \mathrm{mM}$ and $5 \mathrm{mM}$ produced colorless and stable colloids of Ir NPs, but the 1.25 and $2.5 \mathrm{mM} \mathrm{H}_{2} \mathrm{IrCl}_{6}$ solution, in this case, produced very few heterogeneously nucleated particles even after 12 hours. Consequently, incubation time studies were performed only for the $5 \mathrm{mM} \mathrm{H}_{2} \mid \mathrm{rCl}_{6}$ solution. Figure 4.35 shows the results for 12 hour and 24 hour incubation times, respectively. Each figure includes a $5 \mu \mathrm{m} \times 5 \mu \mathrm{m}$ AFM image along with data on the particle size distribution. Table 4.11 summarizes the AFM data, while Table 4.12 summarizes the XPS data for these nucleation conditions. It is immediately clear from the AFM data that the areal density of the particles increases with incubation time as does the surface coverage. However, the mean particle diameter and height remain essentially unchanged at $\sim 50-70 \mathrm{~nm}$ diameter and $\sim 1-3 \mathrm{~nm}$ height. This is also reflected in the XPS data which show that the atomic percent of Ir increases with incubation time.

In summary, the areal density, coverage, and size distribution at 12 hour and 24 hour are essentially in the desired range for sensor development. If sensor development studies require greater coverage, however, higher concentrations of $\mathrm{H}_{2} \mathrm{IrCl}$ may be considered. 

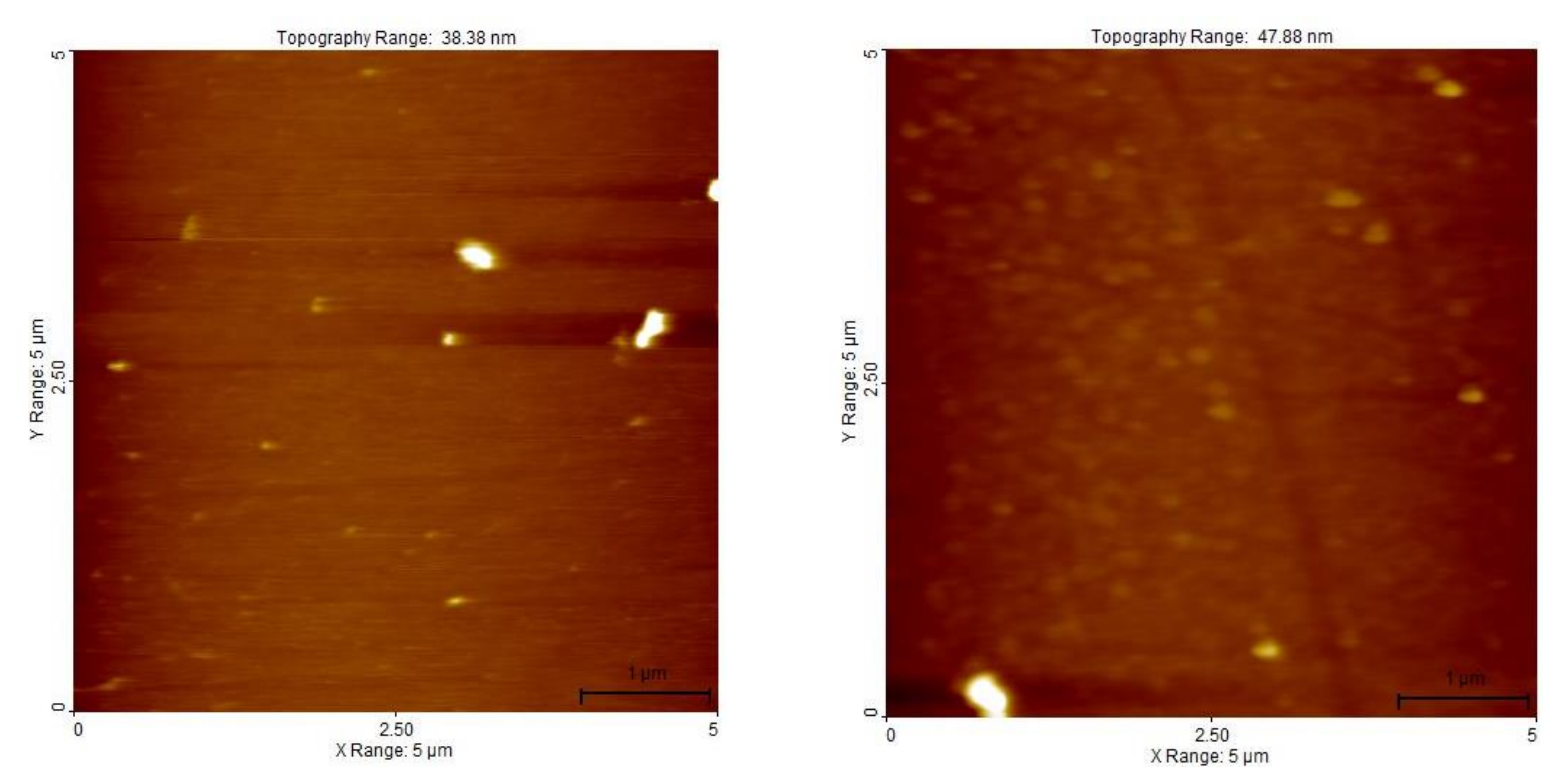

Figure 4.35: AFM images of the IrNPs, obtained with $2.71 \mathrm{mM} \mathrm{NaBH}_{4}$ and $5 \mathrm{mM} \mathrm{H}_{2} \mathrm{lrCl}$, after $12 \mathrm{hr}$ and $24 \mathrm{hr}$ incubation time.

Table 4.11: AFM Analysis for Iridium nanoparticles with $2.71 \mathrm{mM} \mathrm{NaBH}_{4}$ and $5 \mathrm{mM} \mathrm{H}_{2} \mathrm{IrCl}_{6}$, after 12 hour and 24 hour incubation time.

\begin{tabular}{|c|c|c|c|c|}
\hline Time & $\begin{array}{c}\text { Number of } \\
\text { particles }\end{array}$ & Coverage $(\%)$ & $\begin{array}{c}\text { Mean } \\
\text { diameter(nm) }\end{array}$ & Mean height(nm) \\
\hline 12 hour & 77 & 0.7 & 49.4 & 3.62 \\
\hline 24 hour & 275 & 6.37 & 75.1 & 1.83 \\
\hline
\end{tabular}

Table 4.12: XPS Analysis for Iridium Nanoparticles with $2.71 \mathrm{mM} \mathrm{NaBH}_{4}$ and $5 \mathrm{mM} \mathrm{H}_{2} \mathrm{IrCl}_{6}$, after 12 hour and 24 hour incubation time.

\begin{tabular}{|c|c|c|c|c|}
\hline Time & C1s (\%) & O1s (\%) & Si2s (\%) & Ir4f (\%) \\
\hline 12 hour & 42.54 & 29.76 & 27.47 & 0.23 \\
\hline 24 hour & 47.32 & 29.05 & 23.28 & 0.34 \\
\hline
\end{tabular}




\subsection{Deposition of the Device structure}

Device fabrication, as noted previously, consists of graphene growth, particle nucleation and growth and deposition of the device structure. This section describes the last phase, deposition of the device structure. Initially the graphene was patterned using the standard photolithography techniques. This was followed by deposition of electrical contacts, using e-beam evaporation. This overall process involved multiple steps. Thus there were many opportunities for surface contamination, which decreased the quality of graphene. Ultimately, this process was replaced by a lithography free method. Both approaches are described below.

Lithography is one of the most widely used methods for modern integrated circuit manufacturing. Photolithography is the dominant lithographic technique for industrial wafer processing because of its high throughout, repeatability, and flexibility. It involves the transfer of geometric shapes on a photomask to the surface of a wafer by the exposure of photoresist to a light source at a certain wavelength. In a typical photolithography process, a photoresist is spun onto a wafer to form a uniform thin layer (1-2 $\mu \mathrm{m})$ on the surface. A photomask is placed over the resist layer, which is then selectively exposed to light at a certain wavelength (in the range of UV, DUV, or EUV) through openings in the mask. After the resist is developed, the patterns on the photomask are transferred to the resist layer and ultimately to the substrate by a deposition or etching process.

There are two kinds of photo resists: positive and negative. For a positive resist, the exposed region changes its chemical structure so that it becomes more soluble in a developer (selective wet solvent).A negative resist behaves in an opposite manner. That is, in the exposed regions the resist is polymerized and becomes difficult to dissolve in a developer. The developer only removes resist on the unexposed regions.

The patterning of the graphene films in this dissertation was done using a variant of the standard lithography techniques described above to open holes in the photoresist through which metal for the device structure was deposited. The process is known as image reversal and it produces a resist profile with a negative (undercut) wall profile that can be used with the lift off techniques to produce the desired metal pattern on the graphene film. The lithography steps are illustrated in Figure 4.36 and described in detail in Appendix D.1. Going from top to bottom, after the photoresist (AZ 5214) was spun onto the graphene film, UV light $(320 \mathrm{~nm})$ irradiated selected areas through the photomask. The sample was then baked $\left(110^{\circ} \mathrm{C}\right)$ to cross-link the polymer in the exposed regions, which makes these areas resistant to the developer (solvent). The sample was then exposed to UV light ( $365 \mathrm{~nm}$ ) without a mask (flood exposure) to make the reminder of the resist soluble (i.e. AZ 5214 is a positive resist). In the last step, this material 
is removed by the developing agent (AZ $300 \mathrm{MIF}$ ), to produce the pattern shown. It is important to notice the undercut (negative) wall of the remaining resist. This is the key to the metal lit off process which follows. The resulting photoresist film on the surface, as analyzed by an alpha-step profilometer, was found typically to be 1.4-1.5 $\mu \mathrm{m}$ thick.

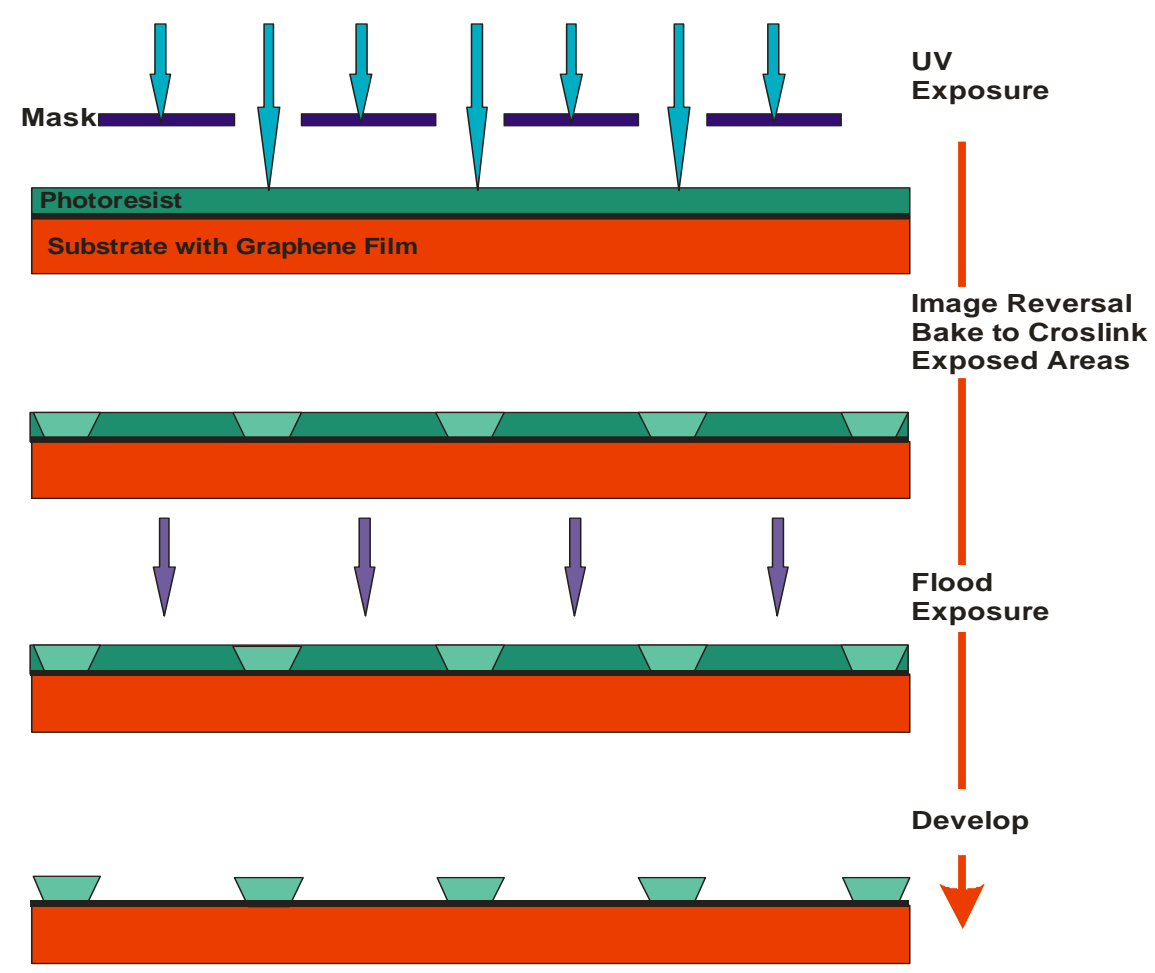

Figure 4.36: Photolithography steps for patterning of the graphene films.

Figure 4.37 shows the optical micrograph of the patterned surface. The light areas are openings in the photoresist which expose the underlying graphene film. The darker region are the remaining photoresist. The large squares are $100 \mu \mathrm{m} \times 100 \mu \mathrm{m}$ and are the areas where the Ti/Au wire bonding pads will be deposited. The small gold squares are alignment marks in the photolithography process. The lines are areas where the Ti/Au electrical interconnects between the graphene and the wire bonding pads will be deposited. The lateral separation between interconnects varies from $20 \mu \mathrm{m}$ to $70 \mu \mathrm{m}$ from right to left.

The next step of in the fabrication process was the deposition of the Ti/Au bonding pads and interconnect ( $100 \mathrm{~nm}$ ) using e-beam evaporation. Following this step, the unwanted metal is lifted off using acetone to dissolve the underlying photoresist. This is possible because the undercut wall of the photoresist protects it from metal deposition and allows the acetone to come in contact with the soluble photoresist. 


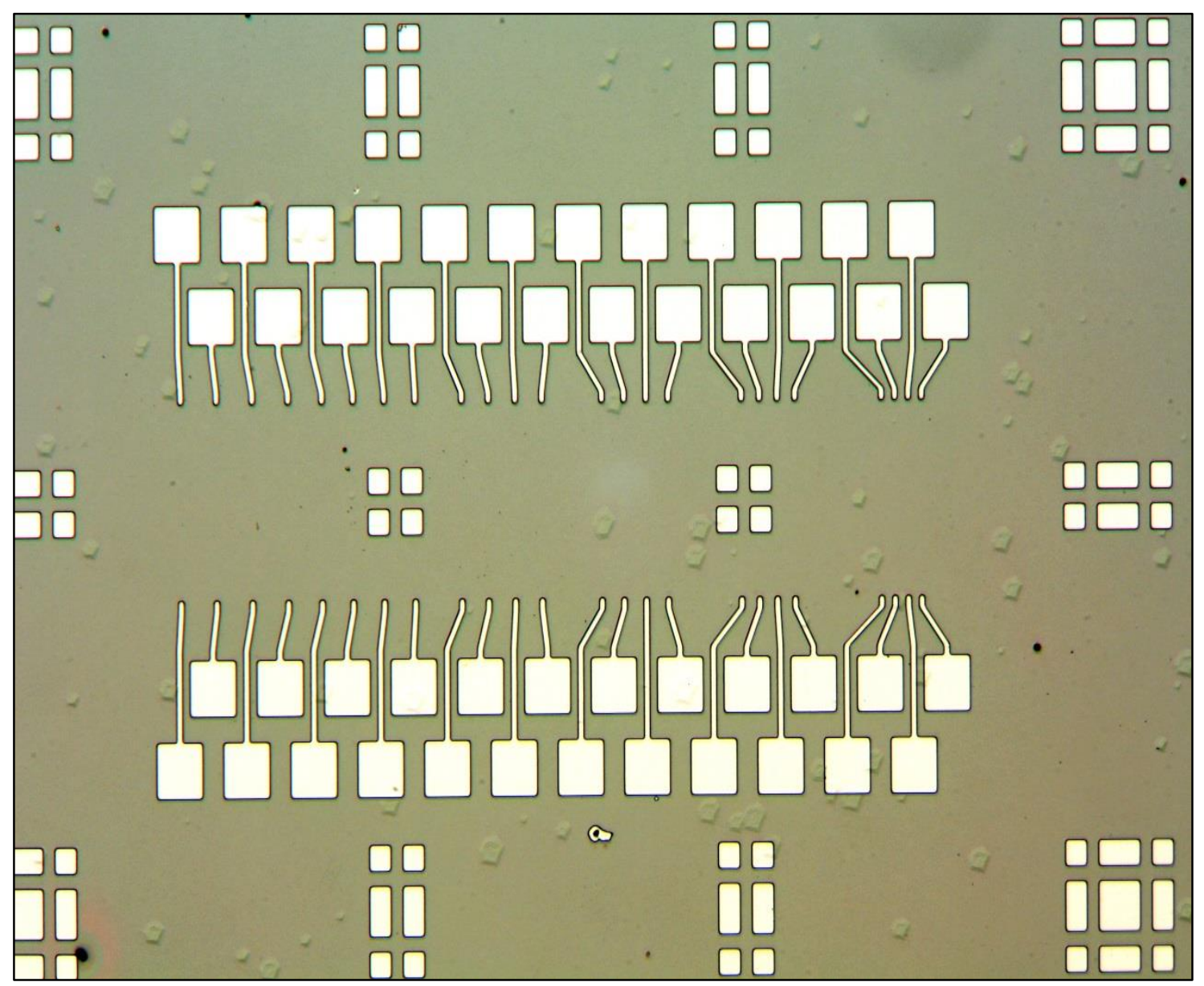

Figure 4.37: Optical micrograph of photoresist with the desired sensor pattern to create contact holes.

The final step involves removal of the graphene from all areas other than the desired current path between gold interconnects. To do this a positive photoresist (AZ 5214) was spun on. Then using a second mask was employed to produce a photoresist strip over the graphene interconnects. Figure 4.38(a) shows the Ti/Au pads and interconnects on the graphene film, and Figure 4.38(b) shows the same area after deposition of the photoresist to protect the underlying area.

The next step was removal of graphene from the unwanted surface of the wafer. This was performed using an $\mathrm{O}_{2}$ plasma, which oxidizes the exposed graphene [108]. The oxidation step was done with ICP/RIE (400 W ICP power, $80 \mathrm{~W}$ RIE power and $25 \mathrm{mTorr}$ of $\mathrm{O}_{2}$ ). The total etch time required to remove the graphene was 60 seconds, as confirmed by the disappearance of graphene peak from C1s XPS spectra. Figure 4.38(c) shows the device after oxidation and removal of the photoresist. 


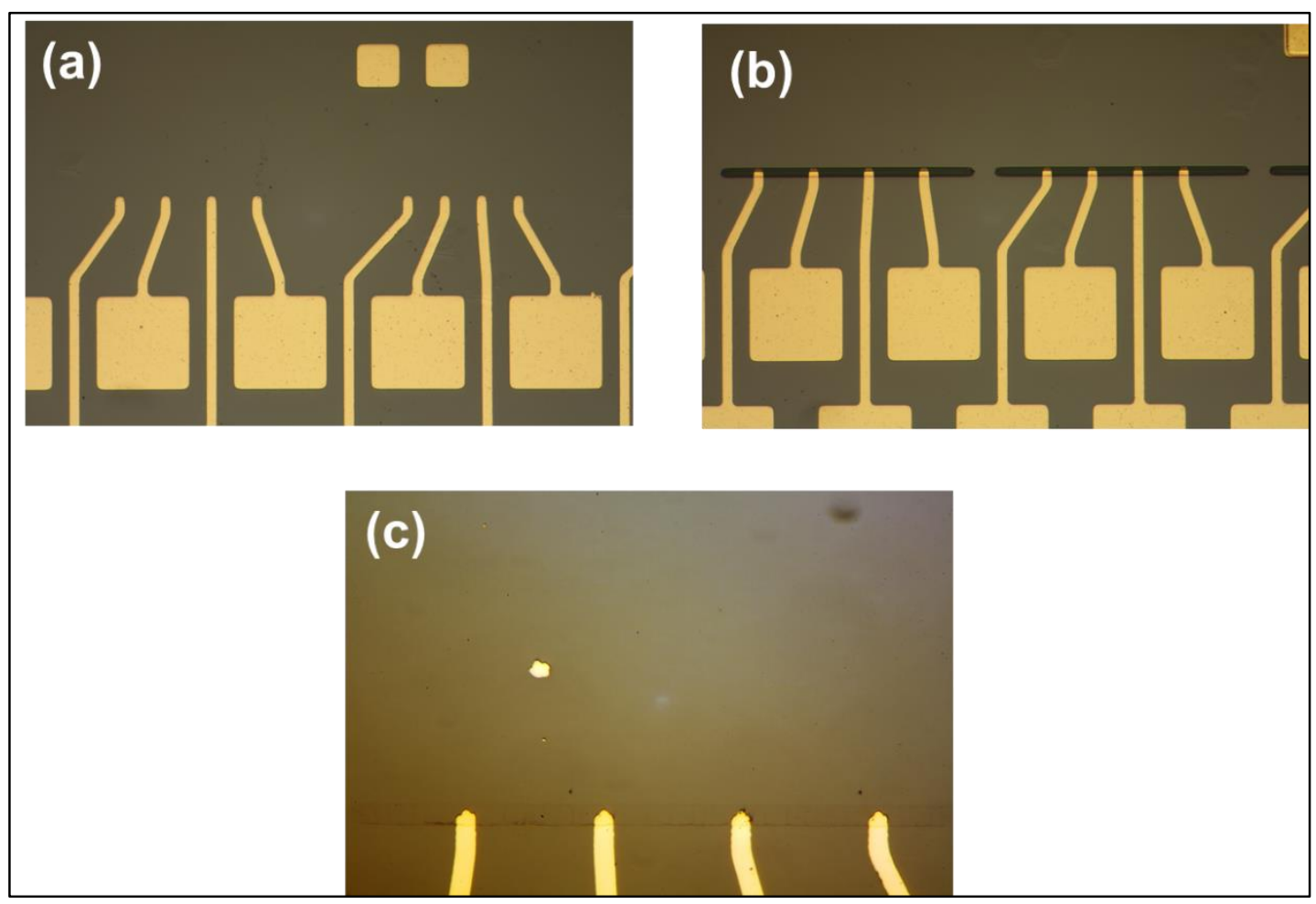

Figure 4.38: Optical micrographs a) After deposition of Ti/Au contacts, b) After deposition of photoresist to protect the graphene strip during an $\mathrm{O}_{2}$ plasma etch to remove the unwanted graphene, c) after $\mathrm{O}_{2}$ plasma etch and photoresist removal.

Even though these methods worked well for most of the samples, the surface of the graphene interacts with the organic photoresist. This has the potential to lead to contamination from either incomplete removal of the photoresist or interaction with the developer or the other various solvents. This is a significant concern, since the adsorption of gases and nucleation of particle growth are both dependent on the graphene surface chemistry.

Although not directly related to the lithography, another problem with the device structure just described involves wire bonding to the Ti/Au bonding pads. Because of the weak bonding between graphene and the metal pads, the wire bonds formed on the pads easily lifted off, usually removing a large portion of the contact pad. Figure 4.39 illustrates the nature of these failures. This type of failure has been reported by other groups who indicate that making "reliable wire bonds to graphene is challenging at best" [109]. This and the surface contamination issue were both resolved using a simpler lithography-free approach described next.

In the lithography-free fabrication approach, the photoresist is eliminated and the pattern is defined by a shadow mask. This is simply a thin metal plate ( $0.005 ")$, with openings "chemically milled" into it. 


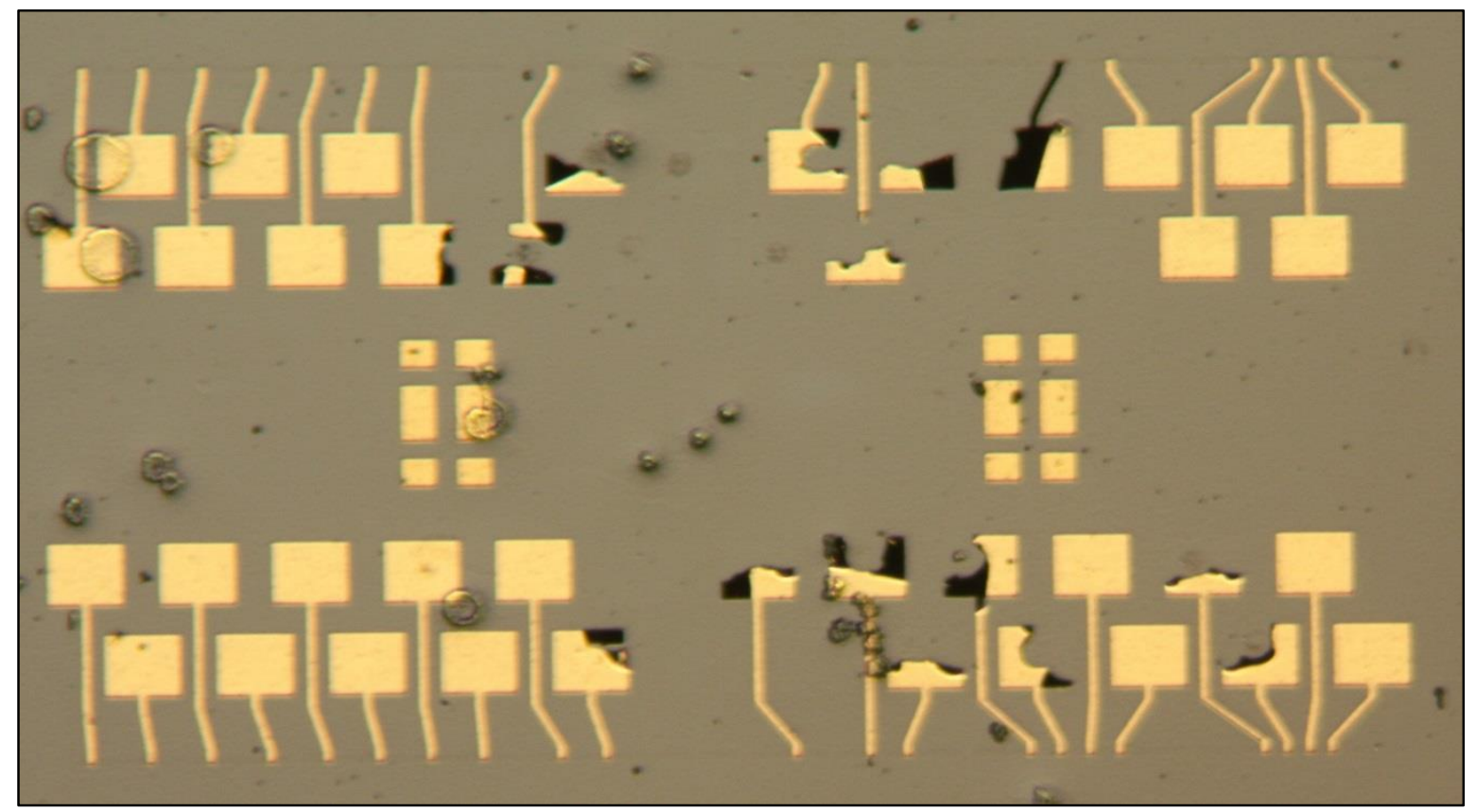

Figure 4.39: Failure of the wire bonds on the graphene surface, resulting in the lift off of gold contacts.

Figure 4.40 shows two shadow masks used in these studies. These were prepared to specs by Photo Sciences Inc. The mask on the left is referred to as the oxide pattern/ mask, while that on the right is called the device pattern/mask. As noted, the dark areas represent openings in the mask. These masks were used to process $1 \mathrm{~cm} \times 1 \mathrm{~cm} \mathrm{SiC} \mathrm{substrates,} \mathrm{diced} \mathrm{from} \mathrm{larger} \mathrm{SiC} \mathrm{wafers.}$

After growth of the graphene films in the $1 \mathrm{~cm} \times 1 \mathrm{~cm} \mathrm{SiC} \mathrm{substrate,} \mathrm{the} \mathrm{oxide} \mathrm{mask} \mathrm{was} \mathrm{placed} \mathrm{over}$ the film and an $\mathrm{O}_{2}$ plasma was used to remove graphene from the open areas of the mask. In fact, a thin
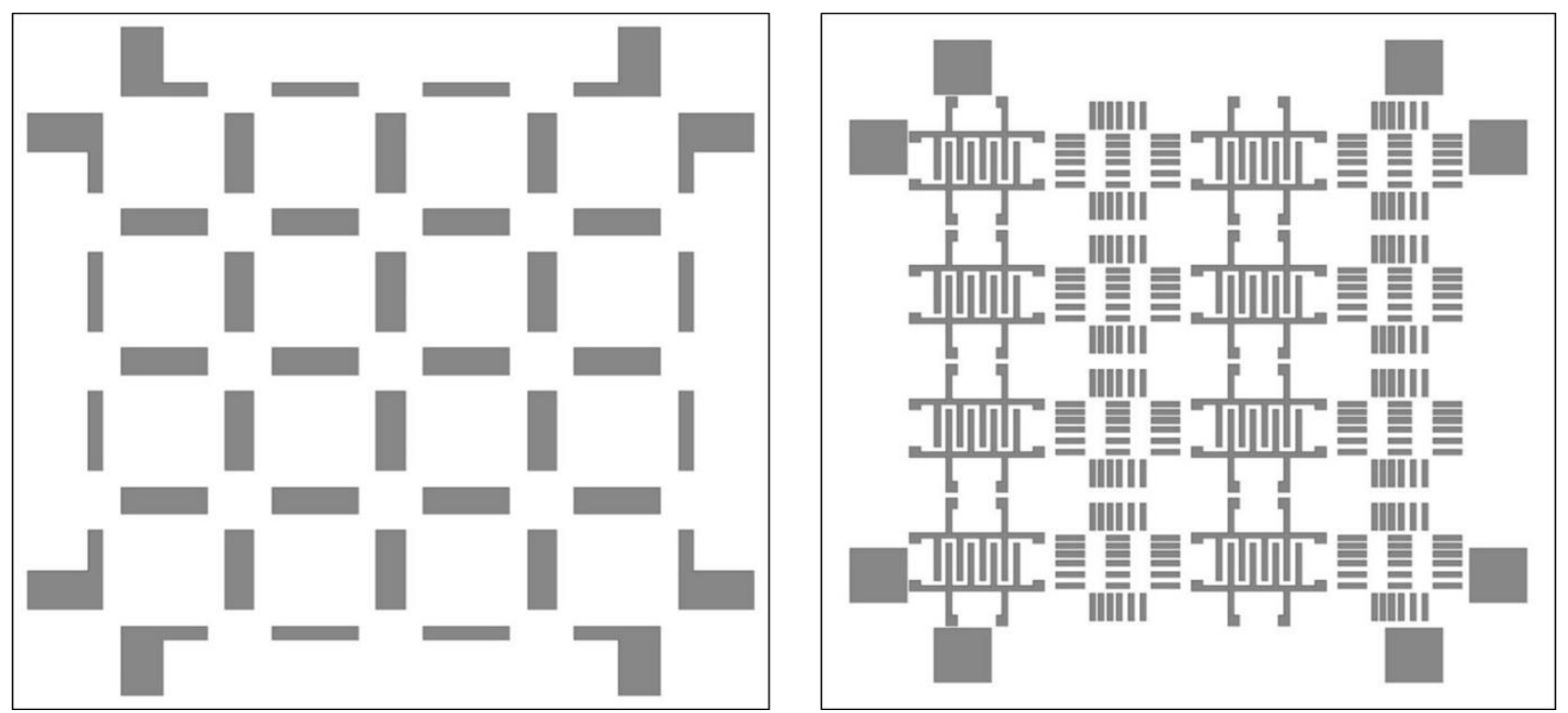

Figure 4.40: Oxygen pattern (left) and device pattern (right) shadow masks used in lithography free fabrication. The dark area represent openings in the sample. 
$\mathrm{SiO}_{x}$ film was formed in these areas. In the next step, the device mask was placed over the substrate and e-beam evaporation was used to deposit the Ti/Au bonding pads and interconnects. When properly aligned, the bonding pads $(100 \mu \mathrm{m} \times 100 \mu \mathrm{m})$ fall on the $\mathrm{SiO}_{\mathrm{x}}$, which allows very robust wire bonds as discussed later. After deposition of the Ti/Au, the $1 \mathrm{~cm} \times 1 \mathrm{~cm}$ sample was diced into $16-2.5 \mathrm{~mm} \times 2.5$ $\mathrm{mm}$ die. Eight of these segments were "sensor structures", while the other eight were simply arrays of metal contacts. The former, as the name implies, were strictly used for sensor testing and characterization, while the latter were used for electrical characterization of the films.

Wire bonding is an essential step in connecting the sensor to external measurement devices. This is typically done by forming either a ball or wedge bond between the pad and an external package using 35 $\mu \mathrm{m}$ gold wire. As noted previously, wire bonds formed on Ti/Au deposited on graphene were unreliable. After some experimentation, it was found that wire bonds formed on $\mathrm{Ti} / \mathrm{Au}$ deposited on $\mathrm{SiO}_{\mathrm{x}}$ were quite robust. This is illustrated in Figure 4.41, which shows a series of successful wire bonds formed on the contact pads on the $\mathrm{SiO}_{\mathrm{x}}$ strip. This was the motivation for using the oxide mask to etch away the graphene and produce the $\mathrm{SiO}_{\mathrm{x}}$.

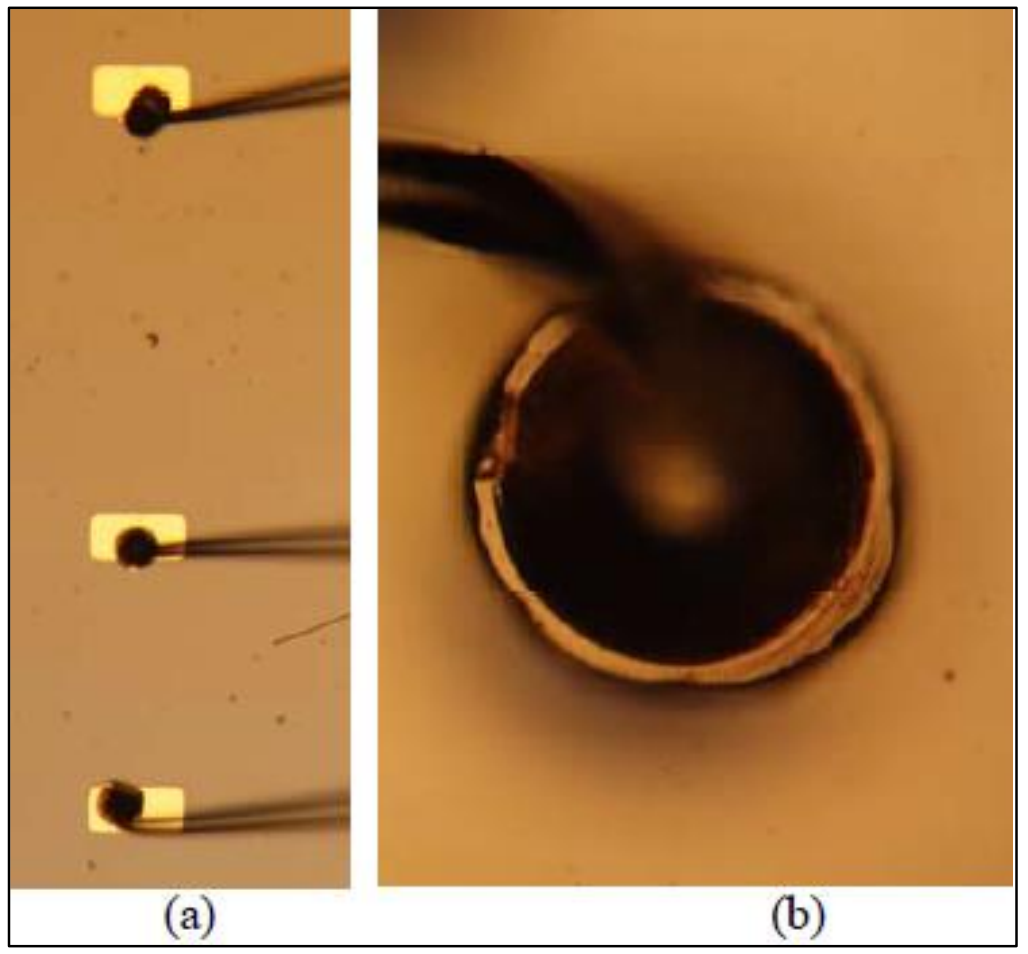

Figure 4.41:(a) A series of successful wire bonds made on bonding pads, located on the $\mathrm{SiO}_{x}$ strip (b) Magnified view of the wire bonds. 


\subsection{Electrical Characterization}

Figure 4.42 below shows the I-V plot for (a)one, (b)two and (c)three layer graphene prepared by etching and ultra-high vacuum annealing at $950^{\circ} \mathrm{C}$, as well as (d)the $\mathrm{SiC}$ surface. These curves exhibit backto-back Schottky behavior, typical of a metal-semiconductor-metal device. This contrasts with exfoliated graphene and graphene formed by Si Sublimation from SiC where the Ti/Au contacts yield Ohmic behavior $[108,109]$. This behavior suggests that the defects have opened a band gap in the normally metallic band structure of graphene. Given the electronegativity of the fluorine atom, it is likely that the defects act as p-type dopants. A second observation from these curves is that over the voltage range considered ( $\pm 3 \mathrm{~V})$, the dominant conduction path is through the graphene rather than the SiC. This is due to the wide band gap of the $6 \mathrm{H}-\mathrm{SiC}(3.05 \mathrm{eV})$.

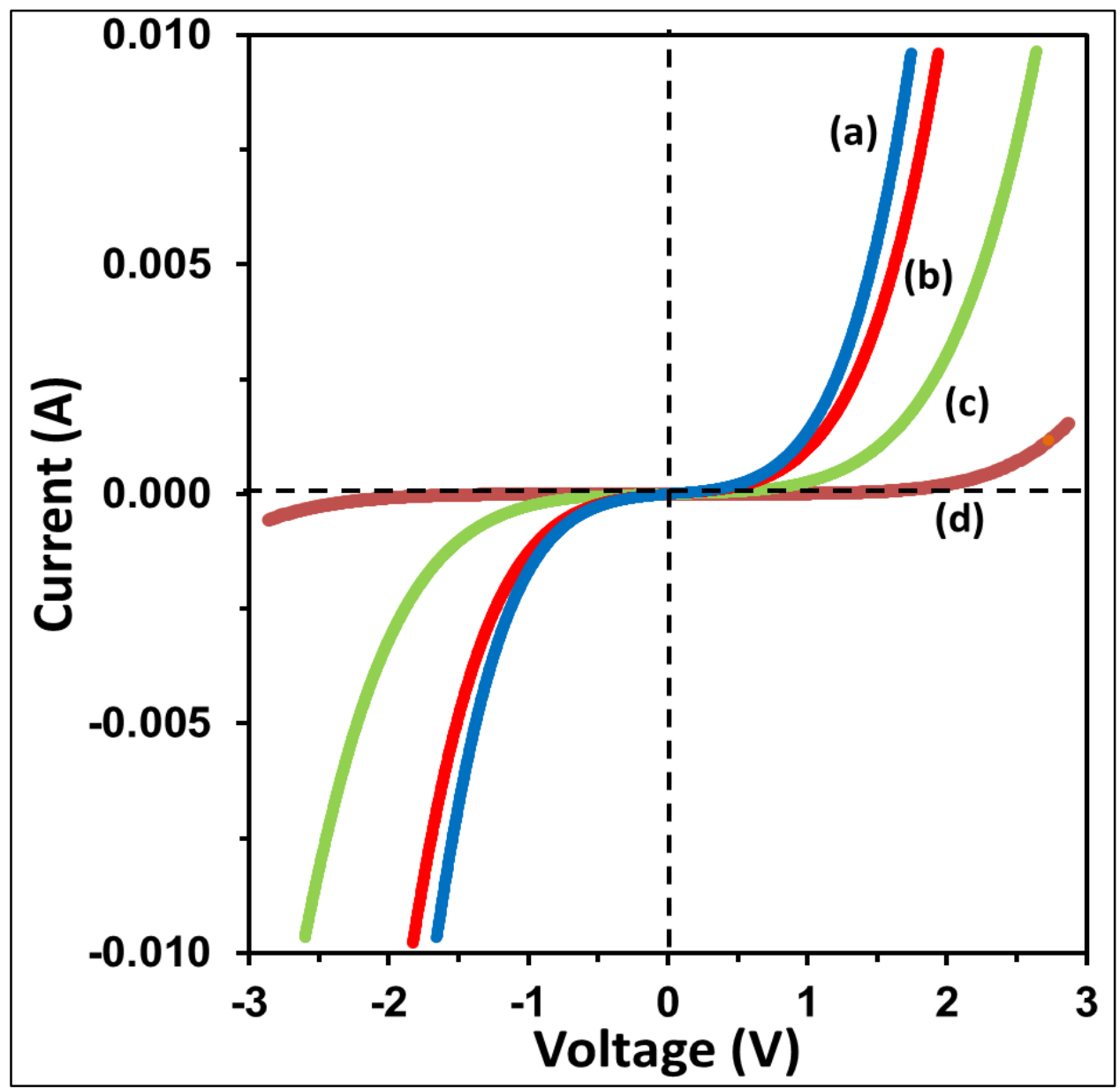

Figure 4.42: I-V plot for a) one, b) two, and c) three layer graphene on SiC films and d) the corresponding response for the SiC substrate. 
The non-linear behavior of the I-V data in Figure 4.42 does not allow a direct calculation of the resistance/conductivity of the film. This is because of the voltage drop across the Schottky barrier of both contacts and the film itself, as noted by Wu and coworkers in their studies of semiconducting graphene oxide films [110]. The response then becomes essentially linear at higher voltages once charge is injected. In this voltage regime, the voltage drop is primarily across the film. Thus, the dynamic resistance/conductivity calculated at high voltage from the plots shown in Figure 4.42 represents the upper/lower limit to the resistivity/conductivity of the film. Following this approach, single, bi and trilayer films produced here were observed to have electrical resistivity values in the range of 1.6-6.4 $\mu \Omega-\mathrm{cm}$. The conductivity values for samples produced using $\mathrm{Cl}_{2}$-based plasma were in the same range [46]. Overall, these values are comparable to those reported by Murali and coworkers for exfoliated graphene nanoribbons [111].

Analysis of the non-linear I-V data was also performed to determine the carrier density $\left(\mathrm{N}_{\mathrm{d}}\right)$ and Schottky barrier height $\left(\Phi_{B}\right)$. Since the graphene film is essentially two-dimensional, the measured current I can be expressed using Anwar's modified Richardson-Duschman equation [112]:

$$
I=\frac{w q \sqrt{8 \pi m^{*}}}{h^{2}}(k T)^{3 / 2} e^{\frac{-q \Phi_{B}}{k T}} e^{\frac{q \Delta \Phi_{B}}{k T}}
$$

where $w$ is the contact width, $q$ the charge present, $m^{*}$ is the charge carrier's effective mass, $T$ is the film temperature, $\mathrm{h}$ is Planck's constant, k is Boltzmann's constant, $\Phi_{B}$ is the Schottky barrier height, and $\Delta \Phi_{B}$ is the barrier lowering due to the applied electric field. Following the treatment given by $\mathrm{Wu}$ and coworkers [109], $\Delta \Phi_{\mathrm{B}}$ can be written

$$
\Delta \Phi_{B} \approx\left(\frac{q^{3} N_{d} V}{8 \pi^{2}\left(\varepsilon_{s}^{\prime}\right)^{2} \varepsilon_{s}}\right)^{1 / 4}
$$

where $\varepsilon^{\prime}$ and $\varepsilon$ are the high frequency and static permittivities, respectively. Substituting this expression into the previous equation and rearranging yields

$$
\ln \left(\frac{1}{w q \sqrt{8 \pi(k T)^{3} m^{*}} / h^{2}}\right)=-\frac{q \Phi_{B}}{k T}+\left(\frac{q^{7} N_{d}}{8 \pi^{2}\left(\varepsilon_{S}^{\prime}\right)^{2} \varepsilon_{s}(k T)^{4}}\right)^{1 / 4} V^{1 / 4}
$$

The term on the left hand side of this equation can be viewed as the natural log of a reduced current. Plotting this term as a function of $\mathrm{V}^{1 / 4}$ should yield a straight line with a slope proportional to the fourth 
root of the carrier density and an intercept proportional to the Schottky barrier height. In these calculations, $q$ was taken as the charge (absolute value) of an electron and $m^{*}$ was taken as the mass of an electron. Although, the values of charge and mass are uncertain, the calculations are not sensitive to specific value chosen. Specifically, variation of two order or magnitudes produce less than $10 \%$ change in calculated values. Values of $\varepsilon^{\prime} \sim 2$ and $\varepsilon \sim 10,000$ were used based on the direct measurements by Huang et al. [113].

A plot of $\ln$ (reduced current) as a function of $\mathrm{V}^{1 / 4}$ for I-V data for two-layer UHV graphene samples is shown in Figure 4.43. Based on the plot, the calculated carrier density is $3.3 \times 10^{14}$, while the Schottky

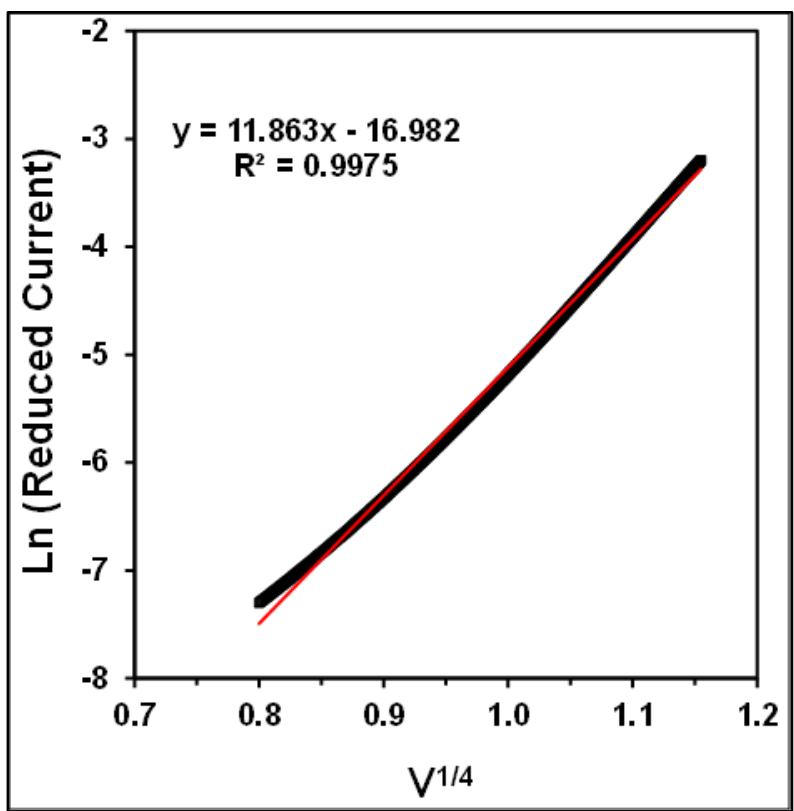

Figure 4.43: Richardson-Duschman analysis of the two layer I-V data.

barrier height is $0.52 \mathrm{eV}$. A summary of the calculated values for one, two and three-layer UHV annealed films is given in Table 4.13 .

Table 4.13: Electrical properties of the UHV annealed graphene films.

\begin{tabular}{|c|c|c|c|}
\hline Number of layers & Resistance $(\mu \Omega)$ & Carrier Density $\left(\mathrm{cm}^{2}\right)$ & Schottky Barrier Height \\
\hline 1 layer & 1.6 & $2.01 \times 10^{14}$ & 0.52 \\
\hline 2 layer & 3.5 & $3.26 \times 10^{14}$ & 0.51 \\
\hline 3 layer & 6.4 & $5.75 \times 10^{14}$ & 0.56 \\
\hline
\end{tabular}


Overall, it can be seen that resistivity and carrier density increase with increasing film thickness, but there is no specific dependence of the Schottky barrier height with thickness. This is most likely due to the fact that the Schottky barrier height reflects the Ti-graphene interface, which should be relatively independent of film thickness.

Figute 4.44 shows the I-V curve for one, two and three-layer films produced by rapid thermal annealing. Based on analysis similar to those for the UHV annealed films, the resistivity, carrier density and Schottky barrier heights were calculated. These results are shown in Table 4.14.

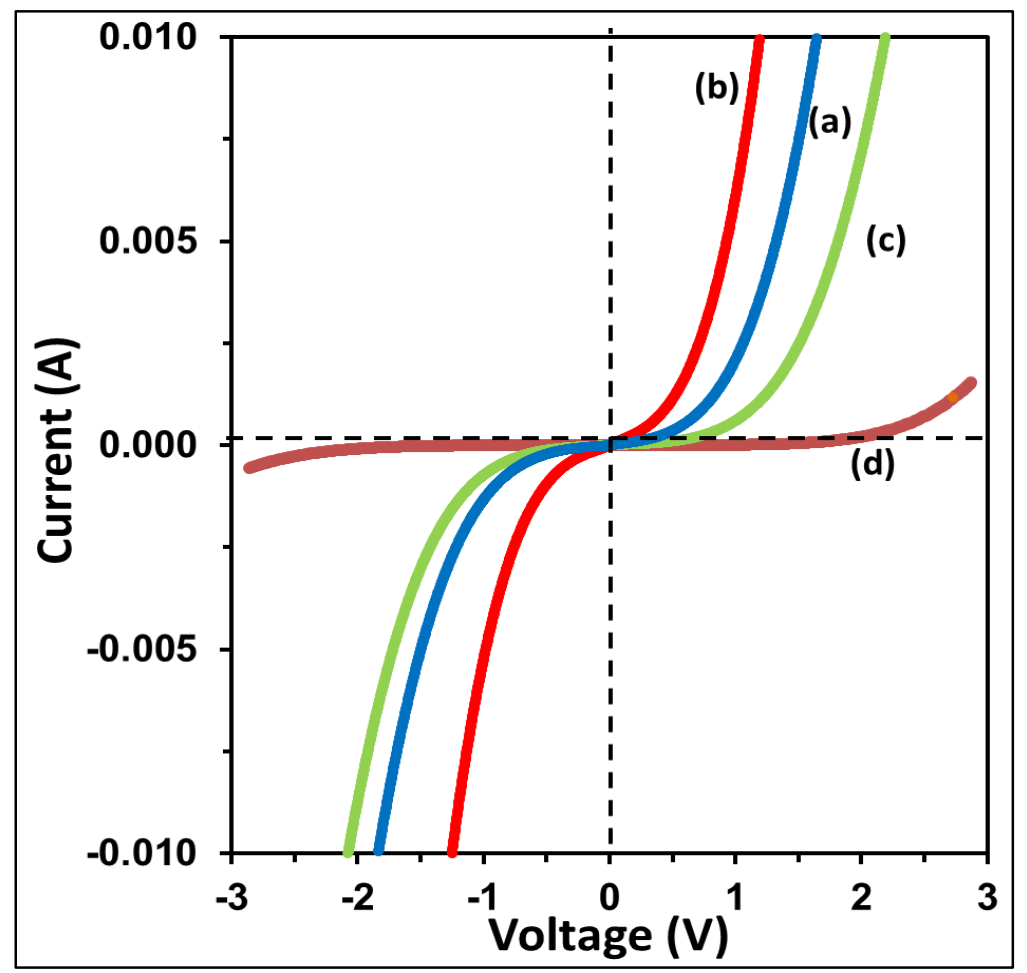

Figure 4.44: I-V plot for a) one, b) two, and c) three layer graphene on SiC films and d) the corresponding response for the SiC substrate, prepared by RTA.

Table 4.14: Electrical properties of the RTA graphene films.

\begin{tabular}{|c|c|c|c|}
\hline Number of layers & Resistance $(\mu \Omega)$ & Carrier Density $\left(/ \mathrm{cm}^{2}\right)$ & Schottky Barrier Height \\
\hline 1 layer & 1.8 & $1.34 \times 10^{14}$ & 0.47 \\
\hline 2 layer & 2.8 & $2.05 \times 10^{14}$ & 0.42 \\
\hline 3 layer & 6.1 & $5.55 \times 10^{14}$ & 0.53 \\
\hline
\end{tabular}


Here it may be seen that the electrical properties for the RTA films are quite comparable to those of the UHV films. This is very advantageous, since the RTA films can by synthesized more rapidly. As noted for the UHV films, these carrier densities represent $5-15 \%$ of the surface atoms which is less than the defects observed using XPS. Thus, it is clear that not all defects can contribute to the conductivity of the graphene.

Reduction in surface defects generally enhances electrical conductivity, which was evident from the electrical characterization of surface modified samples.

Figure 4.45 shows the I-V characteristics of three layer RTA graphene films after being exposed to different liquids, as discussed in section 4.2. Table 4.15 summarizes the key electrical properties of these films. Overall, despite the differences noted in the XPS spectra, the properties of the modified films are remarkably similar to those of the three-layer films. The carrier densities observed here represents 15\% of the carbon atoms on the surface. This is very comparable to the level observed by XPS for the methanol exposed film (14\%), but still slightly less than the values observed for the $\mathrm{HCl}$ soaked RTA films (26\%). It appears that the defects which can be chemically removed are for the most part non-contributors to the carrier density.

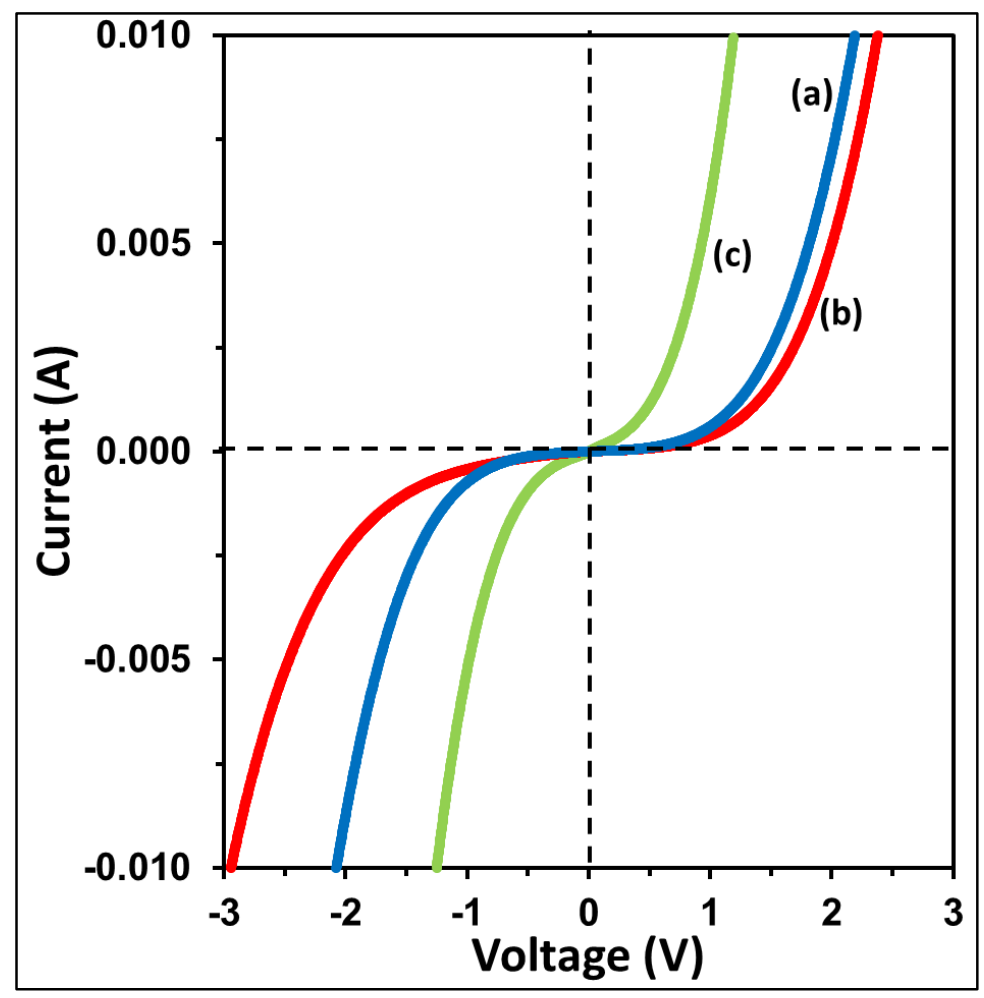

Figure 4.45: I-V plot for a) three layer RTA graphene, b) three layer RTA graphene treated with $1 \mathrm{hr}$ $\mathrm{HCl}$, and c) three layer RTA graphene treated with $12 \mathrm{hr}$ methanol. 
Table 4.15: Electrical properties of the three layer RTA and chemically modified RTA graphene films.

\begin{tabular}{|c|c|c|c|}
\hline $\begin{array}{c}\text { Number of } \\
\text { layers(solution/time) }\end{array}$ & Resistance $(\mu \Omega)$ & Carrier Density $\left(\mathrm{cm}^{2}\right)$ & Schottky Barrier Height \\
\hline 3 layer & 6.1 & $5.55 \times 10^{14}$ & 0.53 \\
\hline 3 layer(HCl/1hour) & 6 & $5.93 \times 10^{14}$ & 0.55 \\
\hline 3 layer(Methanol/12hour) & 5.75 & $5.53 \times 10^{14}$ & 0.54 \\
\hline
\end{tabular}

Figure 4.46 shows the I-V characteristics of two-layer RTA films with Ag, Au, Pt and Ir nanoparticles attached. I-V plot of a two-layer RTA film without nanoparticles is shown for comparison. Table 4.16 summarizes the calculated electrical properties for these films. Here, it can be seen that there is a decrease in the carrier density for the films with attached nanoparticles. Assuming the two-layer film has P-type carriers, this reduction in carrier density could be due to electron transfer from the graphene which results in the annihilation of the holes and consequent increase in resistivity. This is in fact what is observed. Another way to view this is that, in the nucleation process, the electronegative oxygen defects serve as nucleation sites and are converted to $\mathrm{H}_{2} \mathrm{O}$ in the process. Thus dopants are removed by the nucleation process.

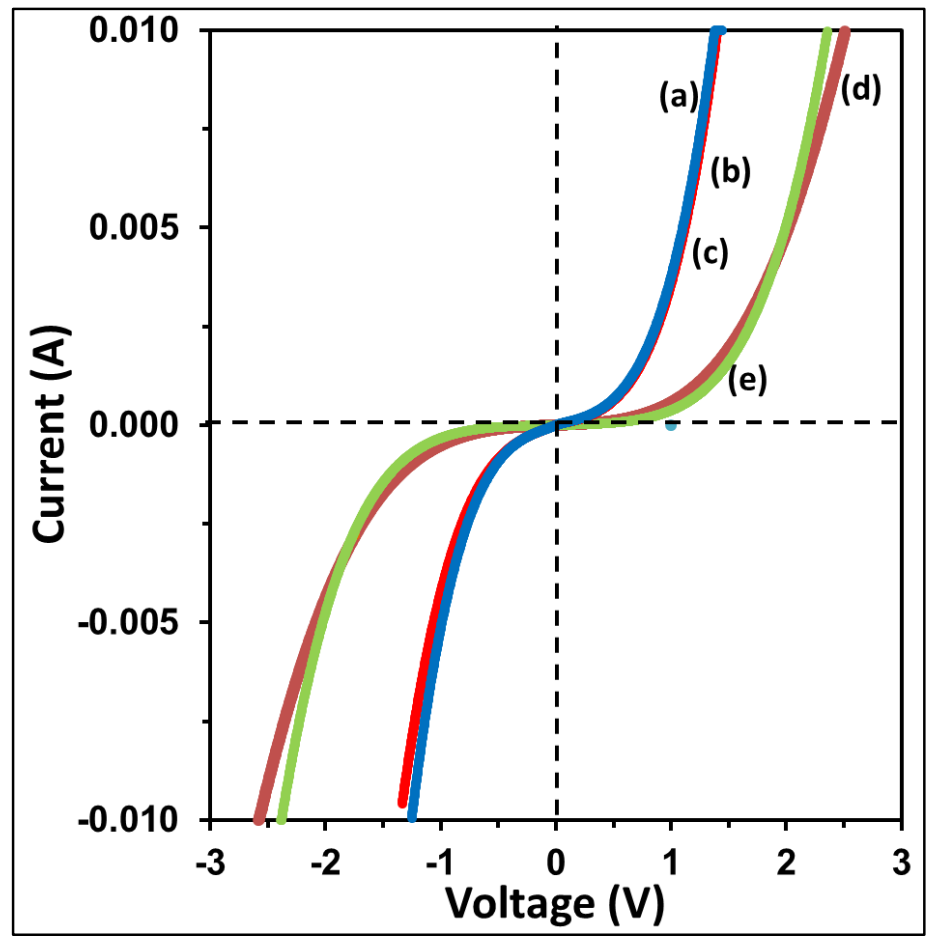

Figure 4.46: I-V plot for (a)two layer RTA graphene, (b) silver(c) gold(d) platinum(e) iridum attached two layer RTA graphene films. 
Table 4.16: Electrical properties of the three layer RTA and nanoparticle attached RTA graphene films.

\begin{tabular}{|c|c|c|c|}
\hline $\begin{array}{c}\text { Number of } \\
\text { layers(Nanoparticle) }\end{array}$ & Resistance $(\mu \Omega)$ & Carrier Density $\left(\mathrm{cm}^{2}\right)$ & Schottky Barrier Height \\
\hline 2 layer & 2.8 & $2.05 \times 10^{14}$ & 0.42 \\
\hline 2 layer(Ag) & 3 & $2.58 \times 10^{14}$ & 0.46 \\
\hline 2 layer(Au) & 3.1 & $2.02 \times 10^{14}$ & 0.43 \\
\hline 2 layer(Pt) & 3.8 & $3.07 \times 10^{14}$ & 0.55 \\
\hline 2 layer(Ir) & 5.5 & $2.32 \times 10^{14}$ & 0.50 \\
\hline
\end{tabular}

\subsection{Initial Sensor Measurements}

The next step was to study graphene sensors in a gas sensing environment. This work was done by Andrew Graves, in the Surface and Material Studies Lab at WVU. For the initial studies of these parameters, sensors were mounted on the sensor platform shown in Figure 4.47. The sensor platform includes the sensor, micro heater, and resistance temperature detector (RTD) mounted on a 16 pin transistor outline (TO) header. The sensor and RTD are glued to the micro heater using a high temperature cement. Micro heater are made by spot-welding their leads to the TO pins. The sensor itself is wire-bonded to the pins. Figure 4.47 (a) shows a paste-up of the platform while Figure 4.47 (b) shows the setup being heated. While rated for only $500^{\circ} \mathrm{C}$, the platform is capable of repeatedly achieving temperatures greater than $700^{\circ} \mathrm{C}$.
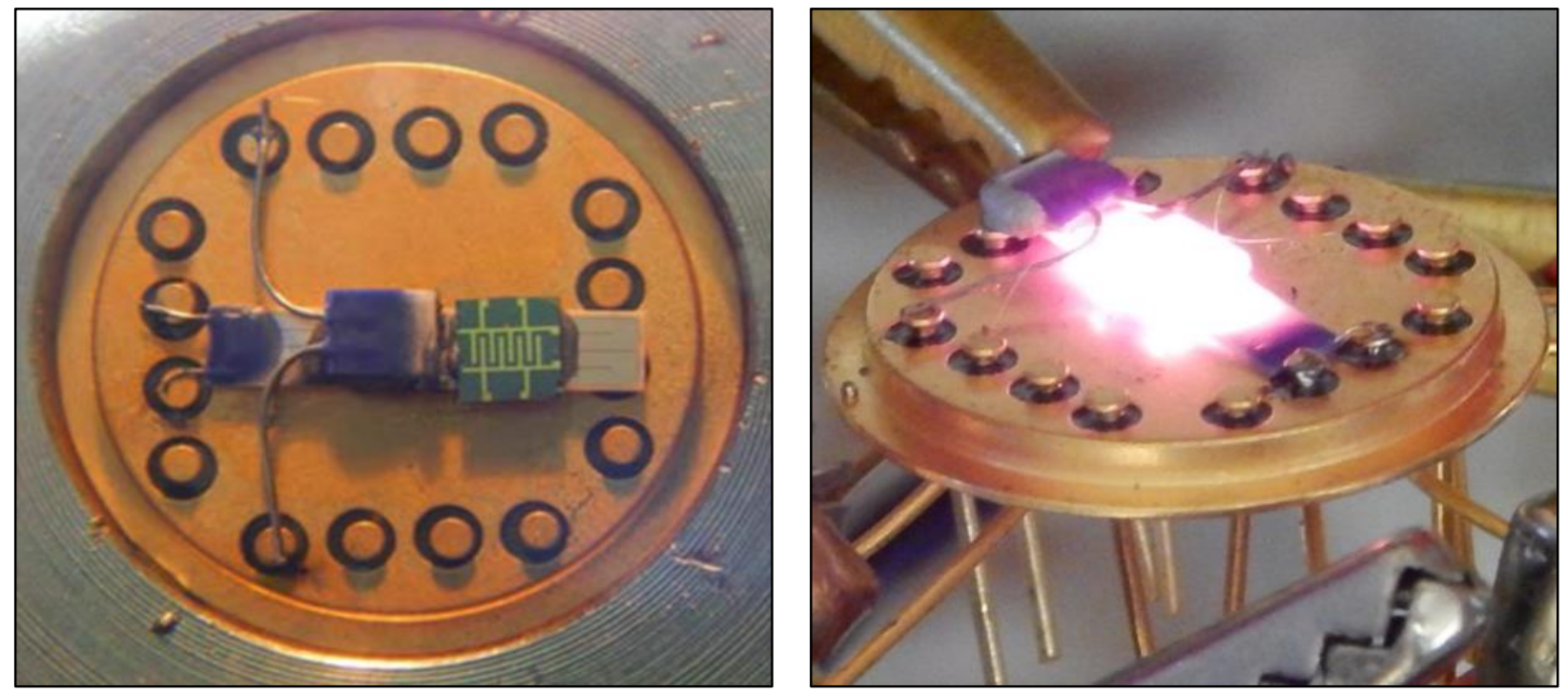

Figure 4.47: a) Paste-up of the sensor platform and b) The sensor platform in operation. 

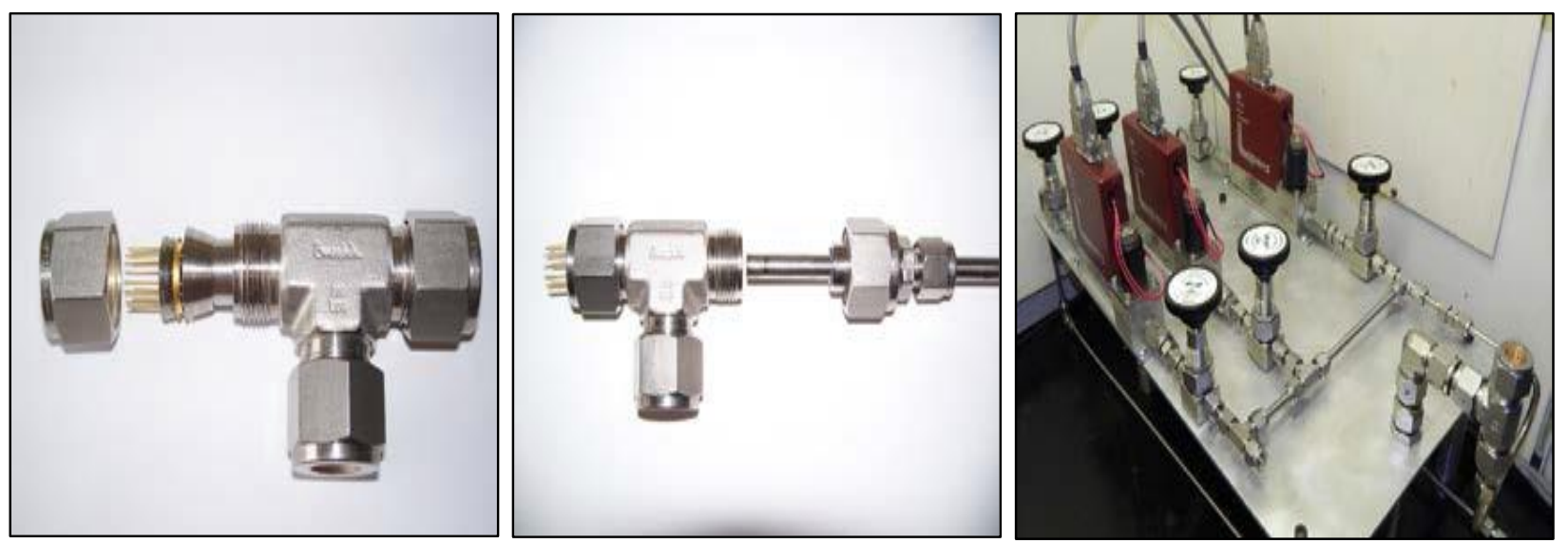

Figure 4.48: a) Sensor platform mounted in the test cell, b) Gas inlet for the test cell, and c) Sensor test unit.

The sensor platform is mounted in the sensor test cell (STC) as shown in Figure 4.48(a). A knife edge milled into the lower (conical) ferule makes a metal-to-metal seal with the TO header when pressure is applied from the nut and upper (ring) ferule. The internal volume of the STC is less than $1 \mathrm{~cm}^{3}$ and, for the mass flow controllers presently mounted, this allows gas residence times of less than 50 milliseconds. These systems are particularly well suited for measurements of sensor response times. As shown in Figure 4.48(b), gases enter the STC coaxially and strike the sensor before being exhausted through the side port of the cell. For gas testing, the STC is mounted on the sensor test unit (STU) as shown in Figure 4.48(c). The STU is setup to handle three gases but can easily be adapted to handle a fourth gas.

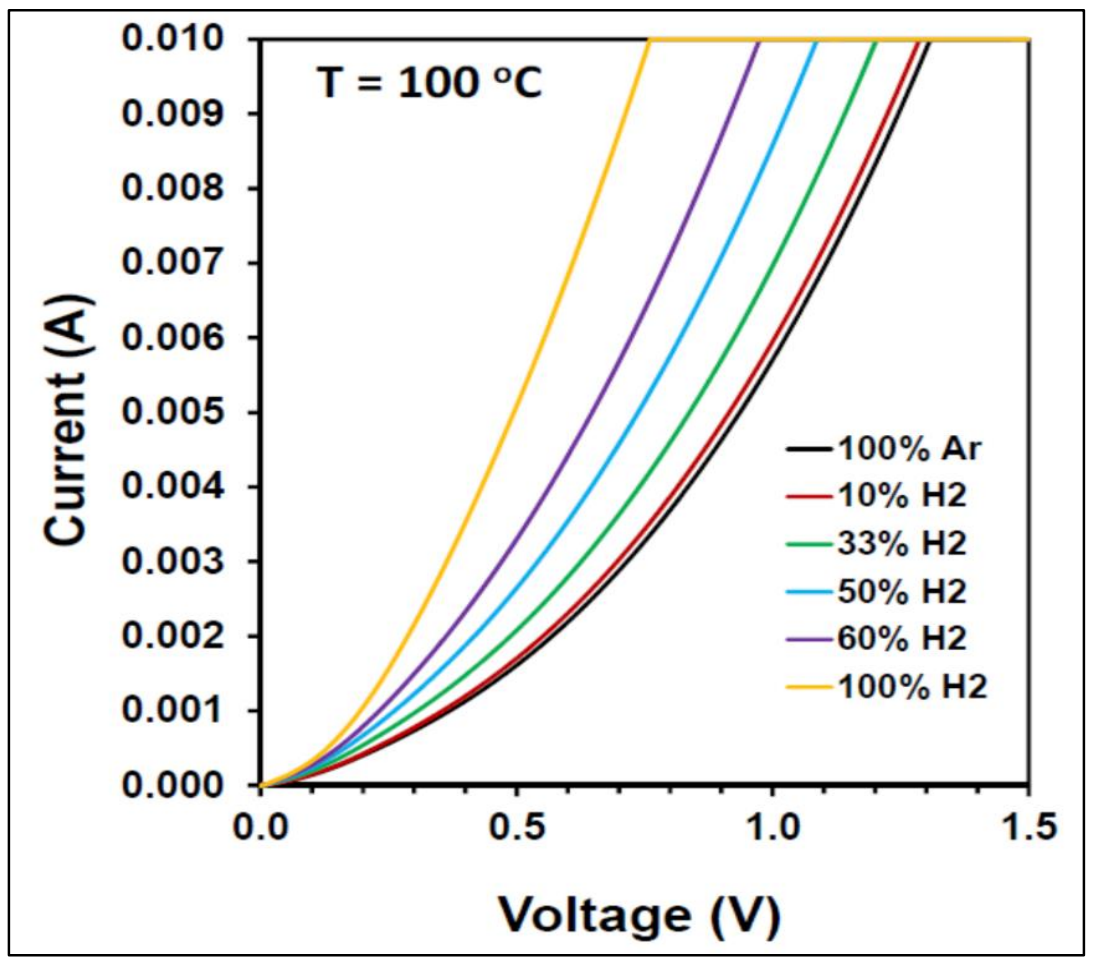

Figure 4.49: Effect of hydrogen composition on I-V characteristics at $100^{\circ} \mathrm{C}$ 
Figure 4.49 shows in greater detail the effects of gas composition on I-V characteristics at a fixed temperature. I-V characteristics showed an increase in conductivity with the increase in the hydrogen gas concentration. These results proved that the graphene sensor are sensitive to the gas composition. The overall behavior seen here for $100^{\circ} \mathrm{C}$ is representative of results at higher temperatures.

The response of the graphene sensor to $\mathrm{H}_{2}$ and $\mathrm{CO}$ adsorption was studied by monitoring the current for a fixed applied voltage in the cold-walled test cell. Preliminary measurements showed that the resistivity of the graphene is a function of gas adsorption and temperature. Figure 4.50 shows the sensor response to alternating $120 \mathrm{sec}$ pulses of $\mathrm{H}_{2}$ and $\mathrm{Ar}$. In this sequence, the sensor was heated to $700^{\circ} \mathrm{C}$ in Ar to establish the baseline. Then the gas flow was switched to $\mathrm{H}_{2}$ for 120 seconds. Six cycles of this type are illustrated in the Figure 4.50 .

Upon exposure to $\mathrm{H}_{2}$, there is a rapid increase in current (becomes more negative) followed by a slower decrease in current. The rapid increase in current is due the chemically induced change in resistivity upon adsorption of $\mathrm{H}_{2}$. The decrease in current is due to cooling of the sensor below the $700{ }^{\circ} \mathrm{C}$ baseline. This change is driven by heat loss to the cold wall as a result of the higher thermal conductivity of $\mathrm{H}_{2}$ relative to Ar. When the flow is again switched to Ar, the temperature, now driven by the heater, increases rapidly due to the lower thermal conductivity of Ar. The temperature slightly overshoots the original 700

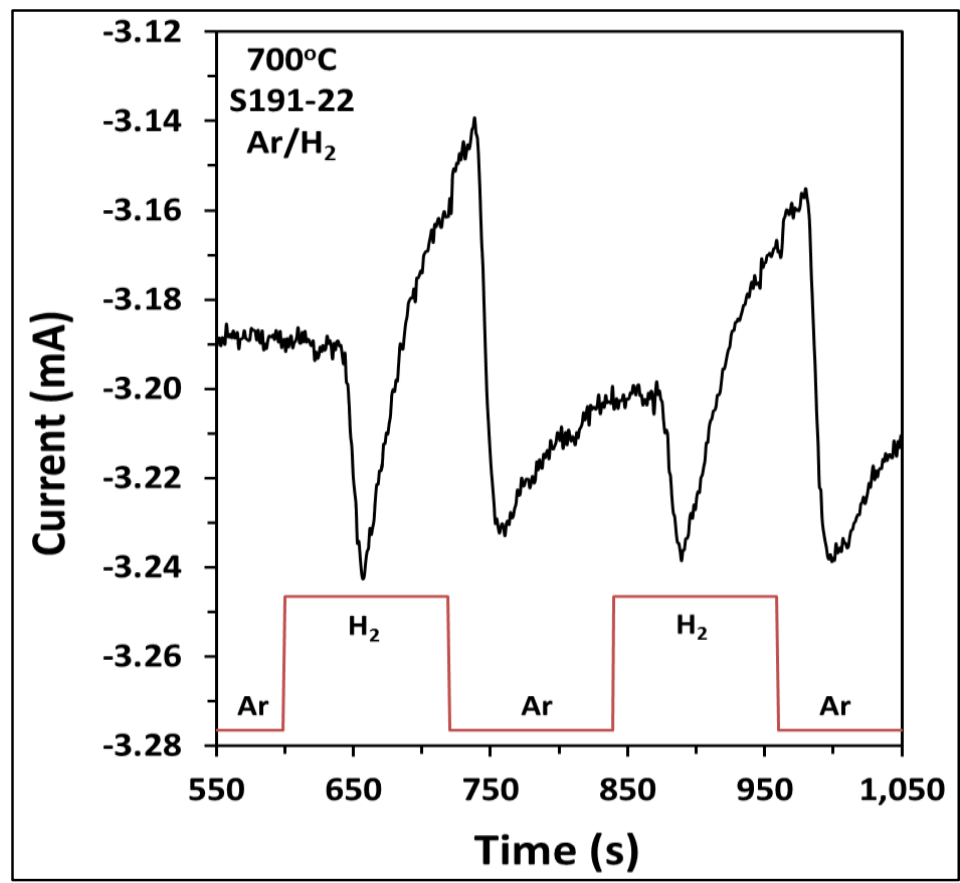

Figure 4.50: Sensor response to alternate $120 \mathrm{sec}$ pulses of $\mathrm{H}_{2}$ and $\mathrm{Ar}$. 
${ }^{\circ} \mathrm{C}$ baseline and then slowly increases as the sensor cools. These studies showed that rapid response times can be achieved using these graphene sensors.

In order to characterize the chemical response, temperature compensated measurements were performed using the heater to maintain sensor temperature regardless of the gas composition. The results of these measurements are illustrated in Figure 4.51 for both $\mathrm{H}_{2}$ and $\mathrm{CO}$. Here the percentage change in sensor current is plotted as a function of applied voltage and temperature. It is clear from these plots that the sensor response is different for both gases. Thus, these graphene sensors was selective to the gases and as per hypothesis, attachment of nanoparticle might result in even better selectivity.
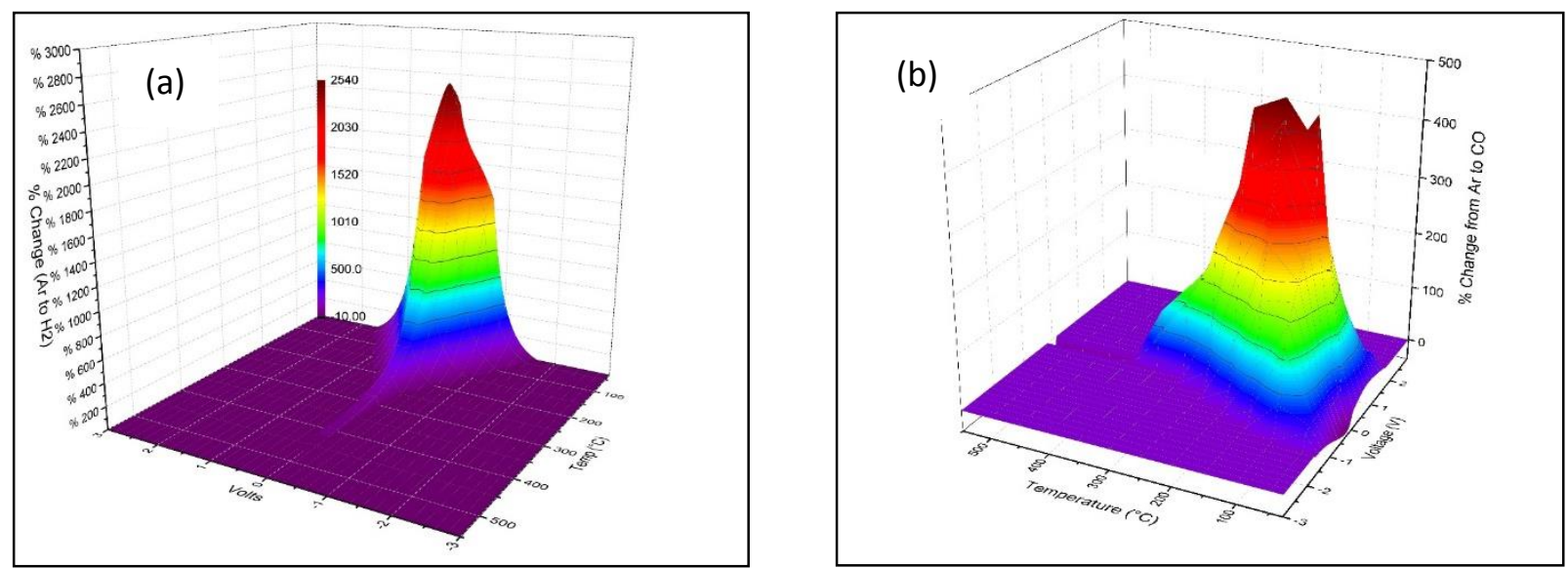

Figure 4.51: Chemical response of the graphene sensor to a) $\mathrm{H}_{2}$ and b) $\mathrm{CO}$ as a function of sensor temperature and applied voltage. 


\section{Chapter 5 CONCLUSION AND RECOMMENDATION}

\subsection{Overview}

Graphene is a two-dimensional structure of $\mathrm{sp}^{2}$ hybridized carbon atoms arranged in a planar hexagonal structure. Because of its unusual mechanical, optical, and electronic properties it has numerous applications. The purpose of this research was to develop graphene and graphene-nanocomposite structures for sensing applications. Inductively coupled-reactive ion etching (ICP-RIE) followed by either ultrahigh vacuum annealing (UHVA) or atmospheric pressure rapid thermal annealing (RTA) in argon was employed to grow large area graphene films on commercial 6H-SiC (0001) substrates. X-ray photoelectron spectroscopy (XPS) determined the number of layers of graphene as well as the chemical nature of the defects. Reflection high energy electron diffraction (RHEED) characterized the crystal quality, and atomic force microscopy (AFM) characterized the surface morphology. In addition, Raman spectroscopy, was used as a basis for comparing these graphene films with graphene produced by other methods. Using solution based methods, procedures were developed for reducing the number of surface defects and nucleating nanoparticles on the graphene surface. Using standard lithography as well as lithography-free methods, procedures were developed for depositing robust, wire-bondable electrical contacts and device patterns on graphene. The electrical properties of these graphene and graphene-nanoparticle composites were characterized using two point current-voltage (I-V) measurements.

\subsection{Conclusions}

Graphene films formed using UHVA were shown to contain fluorine-based defects although some contribution from oxygen-based defects cannot be completely ruled out. In contrast, films formed using RTA have exclusively oxygen-based defects. Based on XPS analyses, the defect levels in the as-prepared UHVA and RTA films were much lower than those found in graphene oxide and were at least as low as those found in reduced graphene oxide. The crystal quality of the UHVA films is quite high as shown by RHEED analyses although the situation is uncertain for RTA films since no RHEED pattern could be obtained. This may be because the RTA films were amorphous, because the films had a small domain size, or because of slightly enhanced surface roughness. Raman spectra for both the UHVA and RTA films were intermediate between graphene and reduced graphene oxide. For both the UHVA and RTA processes, the ICP-RIE bias voltage (as controlled by the RIE power) was shown to provide reproducible control of graphene film thickness. Based on these analyses and the electrical measurements discussed below, both 
UHVA and RTA methods provided comparable films for sensor development. Because the RTA approach was simpler and faster, those films and that approach received the primary focus in subsequent studies.

As determined by XPS, $\mathrm{HCl}$ and $\mathrm{NaBH}_{4}$, both strong reducing agents, and $\mathrm{CH}_{3} \mathrm{OH}$, a protic solvent, were capable of removing significant levels of oxygen-based defects from the RTA graphene surfaces.

One-step solution-based approach were shown to be quite effective for deposition of $\mathrm{Au}, \mathrm{Ag}, \mathrm{Pt}$, and Ir nanoparticles on the RTA graphene surfaces. These method involved dilute $\mathrm{NaBH}_{4}$ in aqueous metal salt solutions. The basic mechanism appears to be heterogeneous nucleation on the graphene surface following a Volmer-Webber growth mechanism. The process was time and concentration dependent. The resulting nanoparticles were typically several nanometers in height and on the order of $50 \mathrm{~nm}$ in diameter. Typical surfaces coverages (based on area) were on the order of $10 \%$. Results suggested that higher coverages could be obtained with longer incubation times and higher salt concentrations.

Conventional photolithography and lithography-free methods combined with e-beam deposition were both effectively used to deposit electrical contacts and device structures on the graphene surface. The lithography-free process had a number of advantages including simplicity as well as reduced potential for surface contamination of the graphene. Using lithography-free methods a reliable process for forming robust wire bondable contacts was developed.

Based on the I-V measurements, both fluorine- and oxygen-based defects appear to open a band gap in the normally metallic graphene band structure. Detailed analyses of the I-V data provided film resistivity, carrier density and Schottky barrier height. In terms of these parameters, the UHVA and RTA films are essentially indistinguishable. In particular, the resistivity values for both UHVA and RTA films were comparable with the nominal value of $1 \mu \Omega \mathrm{cm}$ observed for graphene formed by other methods. This is quite interesting since the defect levels as observed by XPS and the Raman spectra are comparable to reduced graphene oxide which has a resistivity several orders of magnitude higher than this. Although reduction by $\mathrm{HCl}$ and $\mathrm{CH}_{3} \mathrm{OH}$ led to a reduction of oxygen-based defects as determined by XPS, the I-V curves of these films and the corresponding electrical properties were comparable to the original films. This suggests that not all of the defects contribute to the carrier density. The attachment of nanoparticles modifies the electrical properties of the RTA graphene films in some cases and not in other. Nucleation of $\mathrm{Ag}$ and $\mathrm{Au}$ on these films had little effect, while nucleation of Pt and Ir produced a more significant effect on film resistivity. 


\subsection{Significance of current work}

Although there are many methods have been proposed for production of graphene, each comes with certain disadvantages. Mechanical exfoliation is a trial and error method and hence getting high quality graphene is difficult. Sublimation of $\mathrm{SiC}$ requires temperatures as high as $1200^{\circ} \mathrm{C}$ to $1400^{\circ} \mathrm{C}$ and controlling properties and contamination due to instrument material can be difficult. CVD was found to be the best method among all of this, but it requires transfer of graphene on insulating surface before its application in electronics, which might affect quality of graphene. Also, many application requires functionalization of graphene surface using oxygenated or fluorinated functional groups. With the simple two step method described in this dissertation can overcome most of these limitations. As the properties are controlled by user input parameters, graphene with high quality can be made reproducibly. Also, temperatures less than $1000^{\circ} \mathrm{C}$ solves the problem of contamination at higher temperatures. As $\mathrm{SiC}$ is relatively insulating, the graphene can be used directly for electronic applications. As the fluorinated and oxygenated defects are present from the process itself, no further functionalization required for its usage. Thus, a commercially viable process can be designed for graphene production using this method for sensors and various other applications.

\subsection{Recommendation for future work}

The Initial measurement with different gases showed that these graphene-based sensors exhibit different responses in presence of different gases. This shows promise of these materials in sensing applications. The future work will focus on the development of these sensors and characterization. Based on the gases to be detected, appropriate nanoparticles will be chosen and studied for their influence on response time, sensitivity and selectivity. In addition, it is recommended that the use of other SiC substrates (i.e., $4 \mathrm{H}-\mathrm{SiC}$ and semi insulating $4 \mathrm{H}-\mathrm{SiC}$ ) be considered. 


\section{REFERENCES}

[1] K. Novoselov, "Electric field effect in atomically thin carbon films," Science, vol. 306, pp. 666-669, 2004.

[2] B. Brodie, "On the atomic weight of graphite," Philosophical transactions of the Royal Society of London, vol. 149, pp. 249-259, 1859.

[3] "http://www.thejournal.ie/graphene-irish-researchers-major-breakdown-mass-production1424843-Apr2014/," [Online].

[4] V. Gusynin and S. Sharapov, "Unconventional Integer Quantum Hall Effect in Graphene," Phys.REv.Lett., vol. 95, p. 146801, 2005.

[5] H. P. Boehm, A. Clauss, G. Fischer and U. Hofmann, "Surface properties of extremely thin graphite lamellae," in proceedings of the fifth conference of carbon, Heidelberg,Germany, 1962.

[6] K. Kim, Y. Zhao, H. Jang, S. ,. K. J. M. Lee, K. S. Kim, J. H. Ahn, P. Kim, J. Choi and B. H. Hong, Nature, vol. 457, p. 706, 2009.

[7] [Online].Available:http://cnx.org/contents/790bacf3-6512-4957-bbedac887a4fca7c@4/Graphene.

[8] [Online]. Available: http://www.ihp-microelectronics.com/de/forschung/materialien-fuer-diemikro-und-nanoelektronik/projekte/graphene/graphene.html.

[9] T. Denig, "Electrical characterization of buckled graphene films," Dissertation, West Virginia University, Morgantown, 2010.

[10] C. Lee, X. Wei, J. Kysar and J. Hone, "Measurement of the elastic properties and intrinsic strength of monolayer graphene," Science, vol. 321, p. 385, 2008. 
[11] A. Griffith, "The phenomena of rupture and flow in solids," Phil. Trans.R.Soc.London Ser., vol. 221, no. A, p. $163,1921$.

[12] M. Rafiee, "Fracture and fatigue in graphene nanocomposties," Small, vol. 6, pp. 179-183, 2010.

[13] I. Jurewicz, A. Fahimi, P. Lyons, R. Smith, M. Cann, M. Large, M. Tian, J. Coleman and A. Dalton, "Insulator-Conductor Type Transitions in Graphene-Modified Silver Nanowire Networks: A Route to Inexpensive Transparent Conductors," Advanced Functional Materials, vol. 24, p. 7580-7587, 2014.

[14] X. Wang, L. Zhi and K. Mullem, "Transparent,conductive graphene electrodes for dye-sensitized solar cells," Nano Letters, vol. 8, pp. 323-327, 2007.

[15] L. Gomex De Arco, "Continonus, highly flexible,and transparent graphene films by chemical vapor deposition for organic photovoltics," ACS Nano, vol. 4, pp. 2865-2873, 2010.

[16] S. De and J. Coleman, "Are there fundamental limitations on the sheet resistance and transmittance of thin graphene films?," ACS Nano, vol. 4, pp. 2713-2720, 2010.

[17] Q. Su, "Composites of graphene with large aromatic molecules," Advanced Materials, vol. 21, pp. 3191-3195, 2009.

[18] A. Geim and K. Novoselov, "The rise of graphene," Nat Mater, vol. 6(3), pp. 183-191, 2007.

[19] J. Pezoldt, "Top gated graphene transistors with different gate insulators," physical status solidi(c), vol. 7, pp. 390-393, 2010.

[20] W. a. Zhu, "Silicon nitride gate dieletrics and bandgap engineering in graphene layers," Arxiv, 2010.

[21] I. Meric, "Current saturation in zero-bandgap,top agted graphene filed-effect transistors," Nat Nano, vol. 3, pp. 654-659, 2008.

[22] Y. Zhu, "Graphene and graphene oxide:synthsis, properties and applications," Advanced Materials, vol. 22, pp. 3906-3924, 2010. 
[23] Z. Zheng, "Microwave and optical saturable adsorption in graphene.," Nat Phys, vol. 20, pp. 48-51, 20111.

[24] M. Rafiee, "Enhanced mechanical properties of nanocomposites of low graphene content," ACS Nano, vol. 91, pp. 3884-3890, 2009.

[25] F. G. A. Schedin, S. Morozov, E. Hill, P. Blake, M. Katsnelson and K. Novoselov, "Detection of indivisual gas molecules adsorbed on graphene," Nature Materials, vol. 6, p. 652, 2007.

[26] M. Pumera, "Graphene in biosensing," Mater. Today, vol. 14, pp. 308-315, 2011.

[27] Y. Sun, S. Liu, F. Meng, J. Liu, Z. Jin, L. Kong and J. Li, "Metal oxide nanostructures and their gas sensing properties: a review," Sensors, vol. 12, pp. 2610-2631, 2012.

[28] E. Comini, "Metal oxide nano-crystals for gas sensing.," Analytica Chimica Acta, vol. 568, pp. 28-40, 2006.

[29] N. Barsan and U. Weimar, "Fundamentals of metal oxide gas sensors," Tagungsband, pp. 618-621, 2012.

[30] S. e. Kanan, "Semiconducting metal oxide based sensors for selective gas pollutant detection," Sensors, vol. 9, pp. 8158-8196, 2009.

[31] Y. Sandler and M. Gazith, "Surface properties of germanium," The Journal of Physical Chemistry, vol. 63, pp. 1095-1102, 1959.

[32] M. Caroenter, S. Mathur and A. Kolmakov, Metal oxide naomaterials for chemical sensors, Springer, 2013.

[33] S. Sun and P. Wu, "Easy fabrication of macroporous gold films using graphene sheets as a template," ACS Appl. Mater. Interfaces, vol. 5, pp. 3481-3486, 2013.

[34] W. O. R. Hummers, "Preparation of graphitic oxide," J Am Chem Soc., vol. 80, p. 1339, 1985. 
[35] S. Stankovich, A. Dmitriy, D. Richard, A. Kevin, K. Alfred, J. Yuanyuan, W. Yue, T. Sonbinh and S. Rodney, "Synthesis of graphene-based nanosheets via chemical reduction of exfoliated graphite oxide," Carbon, vol. 45, pp. 1558-1565, 2007.

[36] P. Somani and P. Umeno, Chem. Phys. Lett., vol. 430, p. 56, 2006.

[37] W. Bao, F. Miao, Z. Chen, H. Zhang, W. Jang, C. Dames and C. Lau, "Ripple texturing of suspended graphene atomic membranes," arXiv 0903.0413, 2009.

[38] X. Li, W. Cai, J. An, S. Kim, J. Nah, D. Yang, R. Piner, A. Velmakanni, I. Jung, E. Tutuc, S. Banerjee, L. Colombo and R. Ruoff, "Large area synthesis of high quality and uniform graphene films on copper foils," Science, vol. 324, p. 1312, 2009.

[39] S. Park and R. S. Ruoff, Nat. Nanotechnology, vol. 4, pp. 217-224, 2009.

[40] X. Wan, K. Chen, D. Liu, J. Chen, Q. Miao and J. Xu, Chem.Mater., vol. 24, pp. 3906-3915, 2012.

[41] B. Zhang, W. H. P. R. Lee, I. W. Y. Kholmanov, H. Li, H. Ji and R. S. Ruoff, ACS Nano, vol. 6, pp. 24712476, 2012.

[42] S. Bae, H. Kim, Y. Lee, X. Xu, J.-S. Park, Y. B. J. Zheng, T. Lei, H. R. Kim, Y. I. Song, Y.-J. Kim, K. S. Kim, B. Özyilmaz, J.-H. Ahn, B. H. Hong and S. Lijima, Nat. Nanotechol., vol. 5, p. 574, 2010.

[43] A. Van Bommel, J. Crombeen and A. van Tooren, " LEED and Auger-electron observations of SiC (0001) surface," Surface Science, vol. 48, pp. 463-472, 1975.

[44] A. Charrier, A. Coati, T. Argunova, F. Thibaudau, Y. Garreau, R. Pinchaux, I. Forbeaux, J. Debever, M. Sauvage-Simkin and J. Themlin, "Solid-state decomposition of silicon carbide for growing ultrathin heteroepitaxial graphite films," Journal of Applied Physics, vol. 92, p. 2479, 2002.

[45] C. Berger, Z. Song, X. Li, X. Wu, N. Brown, C. Naud, D. Mayou, T. Li, J. Hass, A. Marchenkov, E. Conrad, P. First and W. de Heer, "Electronic confinement and coherence in patterned epitaxial graphene," Science, vol. 312, pp. 1191-1196, 2006. 
[46] S. Raghavan, T. Denig, T. Nelson and C. Stinespring, "Novel surface chemical synthesis route for large area graphene-on-insulator films," J. Vac. Sci. Technol., vol. B30, pp. 1-5, 2012.

[47] Y. Sato, K. Itoh, R. Hagiwara, T. Fukunaga and Y. Itoh, "On the so called "semi-ionic" C-F bond character in fluorine-GIC," Carbon, vol. 42, pp. 3243-3249, 2004.

[48] E. Moreau, R. Ferrer, D. Vignaud, S. Godey and X. Wallart, "Graphene growth by molecular beam epitaxy using a solid carbon source," Phys. Status Solidi, vol. 207, no. A, pp. 300-303, 2010.

[49] R. Pearce, T. lakimov, M. Andersson, L. Hultman, A. L. Spetz and R. Yakimova, "Epitaxially grown graphene based gas sensors for ultra sensitive NO2 detection," Sems. Actuators, vol. 155, no. B, pp. 451-455.

[50] M. Gautam, "Development of graphene based gas sensors," The Univeristy of Toledo, Toledo, 2013.

[51] Y.-J. Kang, J. Kang and K. Chang, " Electronic structure of graphene and doping effect on SiO2," Physical Review B, vol. 78, p. 115404, 2008.

[52] M. Qazi, M. Nomani, V. Shields, M. Spencer and K. , Appl.Phys.Express, vol. 3, pp. 075101-075103, 2010.

[53] J. Dai and J. Yuan, Chem.Phys., vol. 405, pp. 161-166, 2012.

[54] M. Nomani, R. Shishir, M. Qazi, D. Diwan, V. Shields, M. Spencer, G. Tompa and N. Sbrockey, Sens. Actuators B, vol. 150, pp. 301-307, 2010.

[55] X. Wei, Y. Chen, W. Liu and J. Zhong, Phys.Lett.A, vol. 376, pp. 559-562, 2012.

[56] A. Salehi-Khojin, D. Estrada, K. Lin, M. Bae, F. Xiong, E. Pop and R. Masel, Adv.Mater., vol. 24, pp. 53-57, 2012.

[57] M. Chung, D. Kim, H. Lee, T. Kim, J. Choi, S. J. Yoo, S. Hong, T. Kang and Y. Kim, Sens.Actuators B, vol. 166, pp. 172-176, 2012.

[58] F. Yavari and M. Koratlar, J.Phys.Chem.Lett., vol. 3, pp. 1746-1753, 2012. 
[59] G. Ko, Y. Jung, K. Lee, K. Lee and J. Kim, J.Cryst.Growth, vol. 326, pp. 208-211, 2011.

[60] N. Hu, Y. Wang, J. Chai, R. Gao, Z. Yang, E. Kong and Y. Zhang, Sens.Actuators B, vol. 163, pp. 107114, 2012.

[61] V. Dua, S. Surwade, S. Ammu, S. Agnihotra, S. Jain, K. Roberts, S. Park, R. Ruoff and S. Manohar, Angew.Chem.Int.Ed., vol. 49, pp. 2154-2157, 2010.

[62] W. Yuan, A. Liu, L. Huang, C. Li and G. Shi, Adv.Mater., vol. 5, pp. 766-771, 2012.

[63] J. J. Wang, G. P. Yin, H. Liu, R. Y. Li, R. L. Flemming and X. L. Sun, "Carbon nanotubes supported PtAu catalysts for methanol-tolerant oxygen reduction reaction: A comparison between $\mathrm{Pt} / \mathrm{Au}$ and PtAu nanoparticles," J. Power Sources, vol. 194, pp. 668-673, 2009.

[64] A. Kaniyoor, R. Jafri, T. Arockiadoss and S. Ramaprabhu, "Graphene Nanostructured Pt decorated graphene and multi walled carbon nanotube based room temperature hydrogen gas sensor," Nanoscale, vol. 1, pp. 382-386, 2009.

[65] E. Jeon, E. Seo, E. Lee, W. Lee, M. Um and B. Kim, "Mussel-inspired green synthesis of silver nanoparticles on graphene oxide nanosheets for enhanced catalytic applications," Chem. Commun., vol. 49, pp. 3392-3394, 2013.

[66] X. Ran, H. Sun, F. Pu, J. Ren and X. Qu, "Ag Nanoparticle-decorated graphene quantum dots for label-free, rapid and sensitive detection of Ag+ and biothiols," Chem. Commun., vol. 49, pp. 10791081, 2013.

[67] S. Dutta, C. Ray and S. Sarkar, "Silver nanoparticles decorated reduced graphene oxide ( $r G O)$ nanosheets: A platform for SERS-based low level detection of uranyl ion," ACS Appl.Mater.Interfaces, vol. 5, pp. 8724-8732, 2013.

[68] C. J. Murphy, L. B. Thompson, A. M. Alkilany, P. N. Sisco, S. P. Boulos, S. T. Sivapalan, J. A. Yang, D. J. Chernak and J. Y. Huang, "The many faces of gold nanorods," Journal of Phyiscal Chemistry Letters, vol. 1, pp. 2867-2875, 2010. 
[69] L. Gugliotti and D. Feldheim, "RNA-mediated control of metal nanoparticle shape," Journal of American Chemical Society, vol. 127, pp. 17814-17818, 2005.

[70] M. S. Jin, H. Y. Liu, H. Zhang, Z. X. Xie, J. Y. Liu and Y. N. Xia, "Synthesis of Pd Nanocrystals Enclosed by $\{100\}$ Facets and with Sizes $<10 \mathrm{~nm}$ for Application in CO Oxidation.," Nano Research, vol. 4, pp. 83-91, 2011.

[71] J. Petroski, Z. Wang, T. Green and M. El-Sayed, "Kinetically controlled growth and shape formation mechanism of platinum nanoparticles," Journal of Physical Chemistry B, vol. 102, pp. 3316-3320, 1998.

[72] Y. Tang and M. Ouyang, "Tailoring properties and functionalities of metal nanoparticles through crystallinity engineering," Nature Materials, vol. 6, pp. 754-759, 2007.

[73] S. D. Solomon, M. Bahadory, A. V. Jeyarajasingam, S. A. Rutkowsky and C. \& Boritz, "Synthesis and study of silver nanoparticles," J. Chem. Educ., vol. 84, pp. 322-327, 2007.

[74] J. Kimling, M. Maier, B. Okenve, V. Kotaidis, H. Ballot and A. Plech, "Turkevich method forgold nanoparticle synthesis revisited," Journal of Physical Chemistry, vol. 110, pp. 15700-15707, 2006.

[75] G. Frens, "CONTROLLED NUCLEATION FOR REGULATION OF PARTICLE-SIZE IN MONODISPERSE GOLD SUSPENSIONS," Nature-Physical Science, vol. 241, pp. 20-22, 1973.

[76] G. Goncalves, P. Marques, C. Granadeiro, H. Nogueira, M. Singh and J. Grácio, "Surface modification of graphene nanosheets with gold nanoparticles: The role of oxygen moieties at graphene surface on gold nucleation and growth," Chem. Mater., vol. 21, pp. 4796-4802, 2009.

[77] H. Koo, H.-J. Lee, Y. Noh, E. Lee, Y. Kim and W. Choi, "Gold nanoparticle-doped graphene nanosheets: Sub-nanosized gold clusters nucleate and grow at the nitrogen-induced defects on graphene surfaces," J. Mater. Chem., vol. 22, pp. 7130-7135, 2012.

[78] T. S. Ahmadi, Z. L. Wang, T. C. Green, A. Henglein and M. A. ElSayed, "Shape-controlled synthesis of colloidal platinum nanoparticles," Science, vol. 272, pp. 1924-1926, 1996. 
[79] H. Song, F. Kim, S. Connor, G. A. Somorjai and P. D. Yang, "Pt nanoparticles :Shape control and Langmuir-Blodgett monolayer formation.," Journal of Physical Chemistry, vol. 109, pp. 188-193, 2005.

[80] D. Rand and R. Woods, J.Electroanal.Chem., vol. 55, pp. 375-381, 1974.

[81] E. Goldstein, E. Carter and S. Kluz, "The improvement of the oxidation resistance of graphite by composite technique," Carbon, vol. 4, pp. 273-279, 1966.

[82] "http://www.semiconductorwafers.net/SiC-Crystallography--Important-Polytypes-andDefinitions.html," [Online].

[83] C. Peng, "Silicon carbide epitaxial growth using methylsilanes as gas sources," Dissertation, West Virginia University, Morgantown, 2004.

[84] C. D. Stinepspring and J. C. Wormhoudt, "Surface studies relevent to silicon carbide chemcial vapor deposition," J. Appl.phys., vol. 65, pp. 1733-1742, 1989.

[85] "http://www.triontech.com/minilok_phantom.htm," [Online].

[86] [Online]. Available: http://www.cleanroom.byu.edu/sts_icp.phtml.

[87] [Online]. Available: http://nanolab.berkeley.edu/labmanual/chap9/9.06disco.pdf.

[88] F. Reinert and S. Huifner, "Photoemission spectroscopy from early days to recent applications," New J.Phys., vol. 7, p. 97, 2005.

[89] X. Weng, J. Robinson, K. Trumbull, R. Cavalero, M. Fanton and D. Snyder, "Structure of few-layer epitaxial graphene on 6H-SiC(0001) at atomic resolution," Applied Physics Letters, vol. 97, p. 201905, 2010.

[90] M. Seah and W. Dench, "Quantitative electron spectroscopy of surfaces: A standard database for electron inelastic mean free paths in soilds," Surface and Interface Analysis, vol. 1, p. 2, 1979. 
[91] J. Moulder, W. Stickle, P. Sobol and K. Bomben, Handbook of X-ray photoelectron spectroscopy, Physical Electronics,Inc, 1995.

[92] G. Venkataraman, Journey into light: life and science of CV Raman, Indian Academy of Sciences, 1988.

[93] C. Ferrari, J. Meyer, V. Scardaci, C. Casiraghi, M. Lazzeri, F. Mauri, S. Piscanec, K. Jiang, K. Novoselov, S. Roth and A. Geim, Phys. Rev.Lett., vol. 2006, p. 187401, 2006.

[94] [Online]. Available: http://www.edn.com/design/test-and-measurement/4411117/2/Two-wirevs--four-wire-resistance-measurements.

[95] Y. Duan, C. Stinespring and B. Chorpening, "Electronic Structures, Bonding Configurations, and Band-Gap-Opening Properties of Graphene Binding with Low-Concentration Fluorine," Chemistry open, 2015.

[96] S. Luxmi, P. Fisher, R. Feenstra, G. Gu and Y. Sun, "Temperature-dependence of epitaxial graphene formation on $\mathrm{SiC}(0001), "$ J.Electron.Mater., pp. 718-724, 2009.

[97] J. Robinson, X. Weng, K. Trumbull, R. Cavalero, M. Wetherington, E. Franz, M. LaBella, Z. Hughes, M. Fanton and D. Snyder, "Nucleation of epitaxial graphene on SiC(0001)," ACS Nano, vol. 4, pp. 153-158, 2010.

[98] S. Shivaraman, M. V. S. Chandrashekhar, J. J. Boeckl and M. G. Spencer, "Thickness Estimation of epitaxial graphene on SiC gsing attenuation of substrate raman intensity," Journal of Electronic Materials, vol. 38, pp. 725-730, 2009.

[99] J. Rohrl, M. Hundhausen, K. Emtsev, T. Seyller, R. Graupner and L. Ley, "Raman spectra of epitaxial graphene on SiC(0001)," Appl. Phys. Lett., vol. 92, pp. 201918-3, 2008.

[100] J. Robinson, J. Burgess, C. Junkermeier, S. Basedu, T. Reinecke, F. Perkins, M. Zalalutdbiov, J. Baldwin, J. Culbertson, P. Sheehan and E. Snow, Nano Lett., vol. 10, p. 3001, 2010.

[101] R. Nair, "Fluorographene: A two-dimensional counterpart of Teflon," Small, vol. 6, pp. 2877-2884, 2010. 
[102] A. Woodworth and C. Stinespring, "Surface chemistry of Ni induced graphite formation on the 6HSiC (0001) surface: Implications for graphene synthesis," Carbon, vol. 48, pp. 1998-2003, 2010.

[103] S. Stankovich, G. Dommett, K. Kohlhass, E. Zimney, E. Stach, R. Piner, S. Nguyen and R. Ruoff, "Graphene-based composite materials," Nature, vol. 442, p. 282, 2006.

[104] S. Pei and H. Cheng, "The reduction of graphene oxide," Carbon, vol. 50, pp. 3210-3228, 2012.

[105] S. Pei, Z. J., D. J., R. W. and C. H.M., "Direct reduction of graphene oxide films into highly conductive and flexible graphene films by hydrohalic acids," Carbon, vol. 48(15), pp. 4466-74, 2010.

[106] K. Moon, J. Lee, R. Ruoff and H. Lee, "Reduced graphene oxide by chemical graphitization," Nat Comm, vol. 1, pp. 73-78, 2010.

[107] [Online]. Available: http://www.industrial-lasers.com/articles/print/volume-27/issue05/features/micro-processing-with-ultrafast-fiber-lasers.html.

[108] C. Vecchio, S. Sonde, C. Bongiorno, M. Rambach, R. Yakimova, V. Raineri and F. Giannazzo, "Nanoscale Structural Characterization of Epitaxial Graphene Grown on Off-Axis 4H-SiC(0001)," Nanoscale Res. Lett., vol. 6, p. 269, 2011.

[109] H. Wingbrant, H. Svenningstorp, P. Salomonsson, P. Tengström, I. Lundström and A. Lloyd Spetz, "Using a MISiCFET device as a cold start sensor," Sensors and Actutators B, vol. 93, pp. 295-303, 2003.

[110] X. S. Wu, F. Ming, C. Berger and a. d. H. W.A., "The epitaxial-graphene/graphene-oxide junction, an essential step towards epitaxial graphene electronics," Phys. Rev. Lett., vol. 101, pp. 026801-4, 2008.

[111] R. Murli, K. Brenner, Y. Yang, T. Beck and J. Meindl, "Resistivity of graphene nanoribbon interconnects," IEEE Electron Device Letters, vol. 30, p. 611, 2009.

[112] A. Anwar, B. Nabet, J. Culp and F. Casto, "Effects of electron confinement on thermionic emission current in a modulation doped heterostructure," J. Appl. Phys., vol. 85, pp. 2663-2666, 1999. 
[113] X. Huang, C. Zhi, P. Jiang, D. Golberg, Y. Bando and T. Tanaka, "Temperature-dependent electrical property transition of graphene oxide paper," Nanotechnology, vol. 23, p. 455705, 2012. 


\section{Appendix A: Standard Operating Procedure}

\section{A.1 Heater Degas}

1) Make sure the heater connections are secured and thermocouple in place.

2) Turn the power switch to on position.

3) Increase the power using $\mathrm{max} / \mathrm{min}$ buttons on the omega microcontroller with the steps of 5 till you reach the desired temperature. Keep the pressure in the ion gauge below $10^{-8}$ while degassing. This may require reducing power at times and wait between steps.

4) Once the degassing is done, decrease the power, turn off the power and let the sample cool below $100^{\circ} \mathrm{C}$ for sample transfer.

\section{A.2 Sample Transfer}

1) Use a sample holder with 3 pins for transferring the sample from analysis chamber to introduction chamber. With the help of transfer rod, move the holder into the analysis chamber.

2) Adjust the sample with the help of adjustment screws in such a way that the sample is aligned with the external holder. Transfer the sample by rotating the pins so that the sample disc gets locked into the external sample holder.

3) Transfer it into introduction chamber by moving the rod out up to the introduction chamber.

4) To transfer the sample into another holder for further analysis, the sample needs to turn by 90 degrees. This can be achieved with the help of rod which helps to change the sample direction using the hinge joint. Release and control the rod for the movement of hinge by 90 degrees and with the adjustment, adjust the holder in perpendicular directions such that sample can be transferred into that holder with help of pins.

\section{A.3 Load Lock Procedure}

The procedure used for it is known as load-lock procedure as the chamber needs to be brought up to atmospheric temperature from vacuum for sample removal.

\section{Procedure}

1) Close the gate valve (red) to the system.

2) Open the isolation valve to load lock by 13 turns and note the pressure drop on TC gauge. 
3) Start $\mathrm{N}_{2}$ flow to the system by opening the main tank and watch for pressure rise in lon gauge.

4) When nitrogen flow almost stops, remove the $23 / 4$ inch flange.

5) Load and remove the sample with the help of sample holder such that pins of the holder are completely into the grove and lock position.

6) Use new Cu gasket between the flanges and seal the $23 / 4$ inch flange properly such that the bolts are tight.

7) Stop the $\mathrm{N}_{2}$ flow to the system.

8) Open Roughing valve(black valve at the bottom)

9) Start the roughing pump by pressing bottom button on the pump.

10) Wait for system to pump down.

Note: Pressure on TC gauge should fall to $10 \mathrm{mT}$ in 1 or 2 minutes.

11) Wait 30 minutes of the next step till the system pump down well.

12) Close the isolation valve ( 55 inch lb. torque) and immediately (but slowly) open the gate valve. Note any pressure rise on lon gauge.

13) Close roughing valve.

14) Leak check flange by pouring some acetone and check for the sudden pressure change.

Note: If the leak is present, tighten the nuts. If the leak still persists, close gate valve and turn off the roughing pump and wait for roughing turbo to spin down and vent.

15) Turn off the roughing pump and wait for roughing turbo to spin down and vent.

\section{A.4 Reflection High Energy Electron Diffraction (RHEED)}

Reflection high-energy electron diffraction (RHEED) is a technique used to characterize the surface of crystalline materials. RHEED systems gather information only from the surface layer of the sample, which distinguishes RHEED from other materials characterization methods that also rely on diffraction of high-energy electrons.

\section{Operating procedure for RHEED is as described below:}

1) Using the Z-manipulator, make sure that the distance will be $10 \mathrm{~mm}$ so that sample will be properly aligned for the electron beam. Adjust $\mathrm{X}$ and $\mathrm{Y}$ also such that beam is concentrated on sample

2) Start the power button on the RHEED power supply panel. 
3) Start the computer and click on the 'shortcut to KSA 400' icon

4) Click on the e-beam to start the electron beam. Panel for adjusting various voltages, current and focus will appear below.

5) Click on the video icon to start the camera for RHEED image. (Open the black panel and check the camera in case of any problem) and open the shutter to unblock the RHEED screen.

6) Increase the filament current with the step of $1 \mathrm{~A}$ in $10 \mathrm{sec}$ and give some time to stabilize the current. Repeat the procedure the till the current reaches to $1.5 \mathrm{amp}$.

7) Increase the voltage of filament with the step of $2 \mathrm{kV}$ in $10 \mathrm{sec}$ up to the voltage of $13 \mathrm{KeV}$.

8) Once these adjustments are done, change the values of $Z$, focus, $x$ y projection and grid voltage as per the requirement.

9) Typical values for these parameters are $Z=10 \mathrm{~mm}$, Grid voltage $=1.63 \mathrm{~V}$, x-projection $=1.11$ $Y$ projection $=1.5$, focus $=5.0$

10) The adjustments can be done using $X$ and $Y$ screws near the sample adjustment panel to get bright spots.

11) Once the image is obtained, you can save it using file save as command.

12) For more surface sensitive RHEED, the $Z$ value can be decreased. Do this in steps of $1 \mathrm{~mm}$ or so. Eventually the electron beam will be blocked.

13) After all the images are done, click the shutdown button. It will automatically reduce current and voltage value.

14) Close the power button. 


\section{Appendix B: Recipes for Nanoparticle Nucleation}

\section{B.1 Recipe for Silver Nucleation}

A $2.71 \mathrm{mM}$ solution of sodium borohydride $\left(\mathrm{NaBH}_{4}\right)$ was made by dissolving $4.79 \mathrm{mg}$ of $\mathrm{NaBH} 4$ in $47 \mathrm{~mL} \mathrm{DI}$ water immediately before the experiment as a reaction between $\mathrm{NaBH} 4$ and $\mathrm{H}_{2} \mathrm{O}$ occurs and results in the evolution of hydrogen gas decreasing the effectiveness of this reducing agent. A $10 \mathrm{mM}$ stock solution of silver nitrate $\left(\mathrm{AgNO}_{3}\right)$ was made by dissolving $17.0 \mathrm{mg}$ of $\mathrm{AgNO}_{3}$ in $10 \mathrm{~mL}$ of DI water. The substrate was placed with the graphene film facing up in a $50 \mathrm{~mL}$ conical vial or test tube. Then the $\mathrm{NaBH}_{4}$ solution was added to the conical vial before the dropwise addition of $1 \mathrm{~mL}$ of the $\mathrm{AgNO}_{3}$ solution. The substrate remained in solution for 12 hours before being taken out and rinsed with DI water. This silver nucleation was performed on large area and patterned graphene films prepared by RTA.

\section{B.2 Recipe for Gold Nucleation}

A $2.7 \mathrm{mM}$ solution of sodium borohydride $\left(\mathrm{NaBH}_{4}\right)$ was made by dissolving $4.79 \mathrm{mg}$ of $\mathrm{NaBH} 4$ in $47 \mathrm{~mL}$ DI water immediately before the experiment. A $9.6 \mathrm{mM}$ stock solution of auric acid $\left(\mathrm{HAuCl}_{4}+\mathrm{XH}_{2} \mathrm{O}\right)$ was made by dissolving $46.8 \mathrm{mg}$ of $\mathrm{HAuCl}_{4}$ in $12.4 \mathrm{~mL}$ of DI water. The substrate was placed with the graphene film facing up in a $50 \mathrm{~mL}$ conical vial. Then the $\mathrm{NaBH} 4$ solution was added to the conical vial before the dropwise addition of $1 \mathrm{~mL}$ of the $\mathrm{HAuCl}_{4}$ solution. The substrate remained in solution for 12 hours before being taken out and rinsed with DI water. This gold nucleation was performed on large area graphene films prepared by RTA.

\section{B.3 Recipe for Platinum Nucleation}

A $2.7 \mathrm{mM}$ solution of sodium borohydride ( $\mathrm{NaBH} 4)$ was made by dissolving $4.79 \mathrm{mg}$ of $\mathrm{NaBH}_{4}$ in $47 \mathrm{~mL}$ DI water immediately before the experiment. A $5 \mathrm{mM}$ stock solution of chloro platinic acid $\left(\mathrm{H}_{2} \mathrm{PtCl}_{6}+\mathrm{XH}_{2} \mathrm{O}\right)$ was made by dissolving $204.9 \mathrm{mg}$ of $\mathrm{H}_{2} \mathrm{PtCl}_{6}$ in $100 \mathrm{~mL}$ of DI water. The substrate was placed with the graphene film facing up in a $50 \mathrm{~mL}$ conical vial. Then the $\mathrm{NaBH}_{4}$ solution was added to the conical vial before the dropwise addition of $1 \mathrm{~mL}$ of the $\mathrm{H}_{2} \mathrm{PtCl}_{6}$ solution. The substrate remained in solution for 12 hours before being taken out and rinsed with DI water. This platinum nucleation was performed on large area graphene films prepared by RTA. 


\section{B.4 Recipe for Iridium Nucleation}

A $2.7 \mathrm{mM}$ solution of sodium borohydride $\left(\mathrm{NaBH}_{4}\right)$ was made by dissolving $4.79 \mathrm{mg}$ of $\mathrm{NaBH} 4$ in $47 \mathrm{~mL}$ DI water immediately before the experiment. A $5 \mathrm{mM}$ stock solution of chloro iridic acid ( $\mathrm{H}_{2} \mathrm{IrCl}_{6}+\mathrm{XH} 2 \mathrm{O}$ ) was made by dissolving $203.4 \mathrm{mg}$ of $\mathrm{H}_{2} \mathrm{IrCl}_{6}$ in $100 \mathrm{~mL}$ of DI water. The substrate was placed with the graphene film facing up in a $50 \mathrm{~mL}$ conical vial. Then the $\mathrm{NaBH} 4$ solution was added to the conical vial before the dropwise addition of $1 \mathrm{~mL}$ of the $\mathrm{H}_{2} \mid \mathrm{rCl}_{6}$ solution. The substrate remained in solution for 12 hours before being taken out and rinsed with DI water. This iridium nucleation was performed on large area graphene films prepared by RTA. 


\section{Appendix C Atomic Force Microscopy}

Atomic Force Microscopy (AFM) is a form of scanning probe microscopy (SPM) where very high resolution, on the order of fractions of nanometer, can be obtained by scanning a small probe across the sample. Various information regarding material's physical, magnetic or chemical properties as well as surface topography can be achieved by measuring probe's interaction with the surface.

The AFM probe is generally a sharp tip at the end of a small cantilever beam. The probe is attached to a piezoelectric scanner which scans the probe across a selected area of sample. Laser light from a solid state diode is reflected off the back of a cantilever and collected by position sensitive detector consisting of two photo diodes and the deflection information is sent to a computer which generates a topography map or other properties of interest. Imaging of the sample from 100 micrometer to $100 \mathrm{~nm}$ can be done using AFM.

\section{C.1 AFM Operating Procedure}

1) Start the instrument from left to right- picoscan 3000 controller, picoscan molecular imaging microscope and picoplus MAC AC mode controller.

2) Open the software by clicking on the "picoview"icon.

3) Ensure that the scanner's laser spot is aligned to reflect off of the cantilever. Place a white piece of paper under the scanner to make the laser spot visible. Turn on the laser to get a red laser spot on the paper.

4) Adjust the laser with the help of right-to-left and front-to-back knob situated at the top of the assembly in such a way that no red laser spot appears which indicates perfect alignment.

5) Check the alignment by using video system. The spot will not appear when it is exactly above the cantilever.

6) Mount the sample to the sample plate on a magnetically attractive backing which can then be held by the magnet on the standard sample plate. Place the sample plate's front alignment tab over the front alignment pin. Place the second alignment tab over the 
alignment pin and then let the magnets on the three posts gently engage the sample plate to hold it in place.

7) In the Pico View software choose Mode > Contact.

8) Choose Controls > Camera View to open the Camera View video window.

9) Press the Close switch on the HEB to raise the sample until the tip is close to, but not touching, the sample.

10) Viewing the video window, bring the tip and sample very close to contact.

11) Adjust the focus and $x-y$ alignment of the video system such a way that tip is just above the sample surface. This can be done using different knobs for alignments.

12) Locate the area of interest on the sample by manually moving the $X / Y$ stage control micrometers while watching the video window.

13) For tuning the tip, select the view on main menu and select Ac mode controls and adjust the parameters depending on the requirement. Click on the sweep button after that to get the $A C$ mode frequency plot.

14) The resonance frequency of the cantilever is empirically determined by using the complete frequency range (by shifting the frequency slider to minimum and maximum value) and the clicking on start.

15) Resonance peak displayed on the frequency plot is centered and then another frequency sweep is done and the procedure is repeated till the frequency range is less than $10 \mathrm{kHz}$.

16) To make sure that the contact will be gentle, use the deflection pan and adjust to a set point value such that the tip doesn't touch the sample and then click approach which will raise the sample according to set point.

17) Decide the gain value according to the requirement or use default value of $10 \%$.

18) In the scan and motor window, select the scan tab. Enter scan speed and resolution. Typical good values are 1-2 lines/s and 256 respectively.

19) Once all setting is done, click on scan to start the scan and the image can be obtained in the image window. 


\section{C.2 AFM Analysis}

Once images are captured during real-time operation, they can be viewed, modified, and analyzed offline using the software supplied by the AFM manufacturer. Some of the more useful data visualization and processing features for nanoparticle measurements will be discussed here*.

\subsection{Flatten Images}

Usually, the first step in AFM image processing is a line-wise flattening to remove artifacts of the image acquisition process. For instance, samples are not always mounted perfectly perpendicular to the AFM tip, resulting in some tilt that is not actually present on the sample surface. Other sources of artifacts include thermal drift and non-linearity in the scanner. The flattening technique will correct these nonidealities by fitting each scan line with a polynomial and subtracting it from the data. A first order (linear) correction is normally enough to remove any artifacts. We use line-wise levelling for flattening the images

\subsection{Cross-sectional Line Profiles}

Another common feature included in most AFM software packages is the cross-section tool. A cross-sectional line can be drawn across any part of the image, and the vertical profile along that line is displayed. The cursors can be moved to make horizontal, vertical, and angular measurements. By making several cross-sectional line profiles through a nanoparticle, it is not only possible to calculate the particle height, but also to determine if the particle is isolated and sitting on a flat region (e.g., not on a step edge).

\subsection{Height Measurement Procedure}

If the flattening procedure is done properly, the baseline should be relatively flat over the line scan. Subtract the average baseline height from the peak height to find the nanoparticle height. Repeat this procedure for at least 100 nanoparticles for statistical analysis.

\subsection{Automated Batch-Mode Particle Analysis}

SPIP offer an automated particle analysis function. The software can measure the height of particles based on the height of pixel data by using the threshold method and plot a histogram distribution. Prior to performing batch mode measurements, the above mentioned flattening procedure must be applied to ensure a flat substrate. By adjusting the height threshold, the particles above this threshold can be included for analysis, while the particles below this threshold will be excluded. For our 
nanoparticle analysis, we used the dispersed nanoparticle feature as it is best suited for the non-uniform background.

*Obtained from http://www.nist.gov/manuscript-publication-search.cfm?pub_id=912221 


\section{Appendix D Lithography Steps}

\section{D.1 Steps for Image Reversal Process}

1) Clean the sample with acetone $(5 \mathrm{~min})$, followed by methanol $(5 \mathrm{~min}$ ) to remove any particulate before photoresist deposition.

2) Heat the sample at $110^{\circ} \mathrm{C}$ for 2 min to remove water vapor completely.

3) Spin the AZ5214 photoresist with $500 \mathrm{rpm}$ for $5 \mathrm{~min}$ (for better distribution of photoresist), followed by $4000 \mathrm{rpm}$ for $40 \mathrm{~min}$ for 1.5 micron thick photoresist layer.

4) Soft bake at $100^{\circ} \mathrm{C}$ for $1 \mathrm{~min}$

5) Align substrate with the mask using a Suss Micro Tec MA6 mask aligner, followed by exposing it to UV light $(320 \mathrm{~nm})$ for required time (50/UV intensity (sec)) to fix pattern

6) Hard bake at $110^{\circ} \mathrm{C}$ for 1 min for hardening the photoresist.

7) Flood the surface with UV light (365 nm) for desired time. (1000/UV intensity (sec))

8) Develop the sample with AZ 300 MIF developer for $30 \mathrm{sec}$ followed by cleaning by water for $10 \mathrm{sec}$.

\section{D.2 Steps for Photoresist Strip using Photolithography}

1) Clean the sample with acetone $(5 \mathrm{~min})$, followed by methanol $(5 \mathrm{~min}$ ) to remove any particulate before photoresist deposition.

2) Heat the sample at $110^{\circ} \mathrm{C}$ for 2 min to remove water vapor completely.

3) Spin the AZ5214 photoresist with $500 \mathrm{rpm}$ for $5 \mathrm{~min}$ (for better distribution of photoresist), followed by $4000 \mathrm{rpm}$ for $40 \mathrm{~min}$ for 1.5 micron thick photoresist layer.

4) Soft bake at $100^{\circ} \mathrm{C}$ for $1 \mathrm{~min}$

5) Align substrate with the mask using a Suss Micro Tec MA6 mask aligner, followed by exposing it to UV light $(320 \mathrm{~nm})$ for required time (50/UV intensity (sec)) to fix pattern

6) Develop the sample with AZ 300 MIF developer for $60 \mathrm{sec}$ followed by cleaning by water for $20 \mathrm{sec}$. 